A Dissertation
presented to
The Faculty of the Graduate School
At the University of Missouri-Columbia
In Partial Fulfillment
of the Requirements for the Degree
Doctor of Philosophy
DECEMBER 2013
Dr. Randy Curry, Dissertation Supervisor
by


(C) Copyright by Adam Lodes 2013

All Rights Reserved 
The undersigned, appointed by the Dean of the Graduate School, have examined the dissertation entitled

\section{SHOCK WAVE INTERACTION WITH A TOROIDAL AIR PLASMA}

Presented by Adam Lodes, a candidate for the degree of Doctor of Philosophy in Electrical and Computer Engineering,

And hereby certify that, in their opinion, it is worthy of acceptance.

Dr. Randy D. Curry

Dr. Gregory Triplett

Dr. Mark A. Prelas

Dr. Robert Druce 


\section{DEDICATION}

This work is dedicated to those who have supported me throughout my academic career. I am especially grateful to my parents, Donald and Joan Lodes, my brother, Andy Lodes, my loving girlfriend, Audrey Spalding, and all my close friends for their support and encouragement through this process. 


\section{ACKNOWLEDGEMENTS}

First, I would like to express my thanks to Dr. Randy Curry for this opportunity and his continued efforts in this research program. Dr. Curry has continually pushed me to do more and he has heavily influenced my education in this field of research. I also would like to recognize the contributions of Michael Rudroff, who is pursuing his M.S. for related research and assisted greatly in the construction of the test bed and collection of the data on this program. Additional contributions have been made by numerous undergraduate research assistants as well as technicians Scott Booska and Mark Nichols.

I am also grateful for the less formal education gained through working with fellow graduate students throughout my academic career. In particular, I owe thanks to Peter Norgard, Chris Yeckel, Kevin O’Connor, Kelton Clements, Nate Kinsey, and Chris Lapp.

Linda Macon, Mike Fulca, and Vicki Edwards are appreciated for their aid in procurement and all other administrative support. Special gratitude must go to Bill Carter for his role as laboratory supervisor and Dennis Pease for his assistance in the spectroscopy efforts. I would like to acknowledge all other staff and students at the Center for Physical and Power Electronics at the University of Missouri.

I would also like to thank the other committee members, Dr. Gregory Triplett, Dr. Mark Prelas, and Dr. Robert Druce for their service on the committee.

Additionally, this program was partially funded by ONR under grant number: Prime Contract \# N000014-09-C-0568, Plasma Shock Wave Mitigation ARA Subcontract \#S-00296.00001.UMC 


\section{TABLE OF CONTENTS}

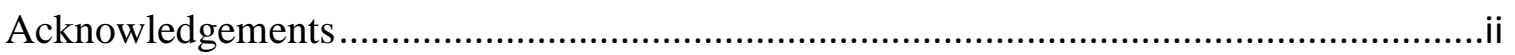

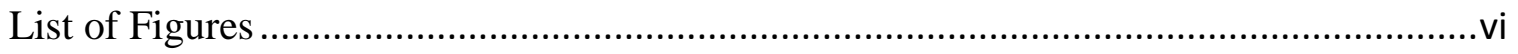

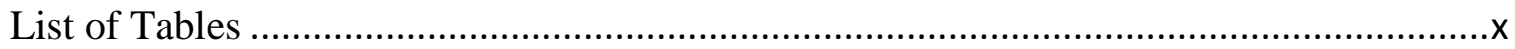

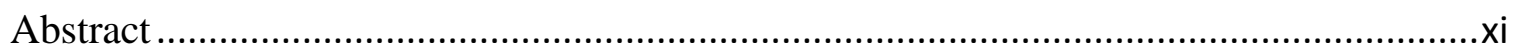

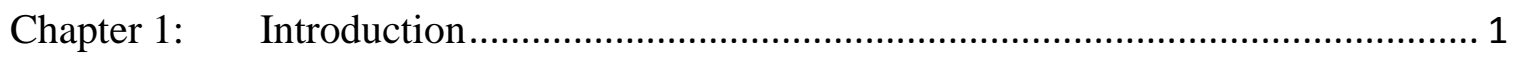

1.1 Background on the Interaction of Plasmas and Shock Waves............................. 5

1.1.1 Wind Tunnel Experiments ............................................................. 5

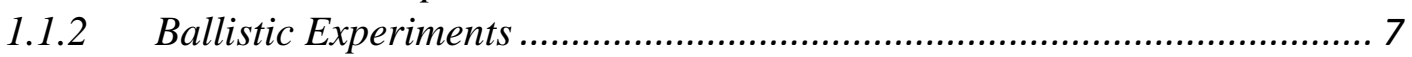

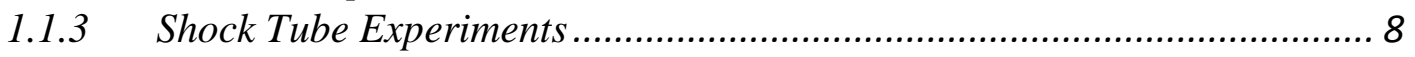

1.1.4 Physical Mechanisms of Mitigation ...................................................... 12

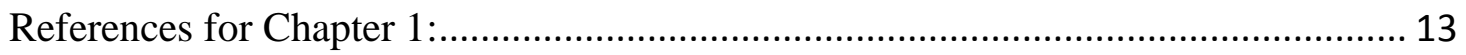

Chapter 2: Plasma Source Investigation ............................................................. 15

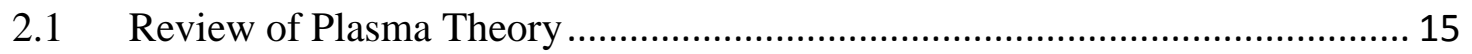

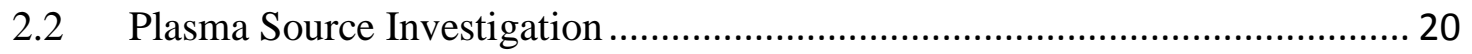

2.2.1 Microhollow Cathode Discharge ......................................................... 21

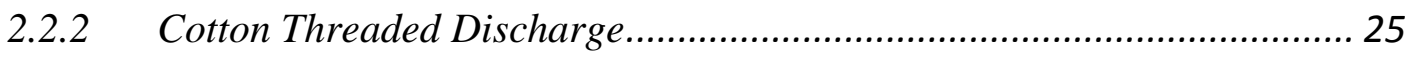

2.2.3 Exploding Wire Plasma Sources............................................................. 27

2.2.4 Early Experiments with the Toroidal Air Plasma................................... 31

2.2.5 Additional Plasma Source Experiments and Discussion on the Selection of the TAP for Interaction Experiments …………………...................................... 34

2.3 Previous Research Related to the TAP Discharge ............................................ 36

References for Chapter 2:................................................................................ 41

Chapter 3: Experimental Test Bench Including the Plasma Circuit and Shock Tube.45

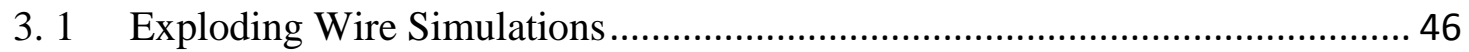

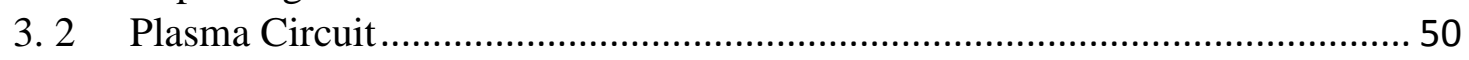

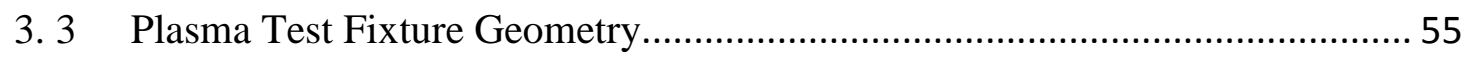

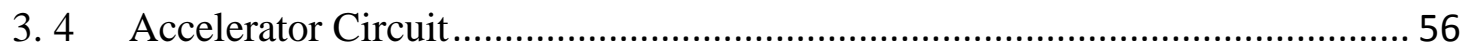

3. 5 Exploding-Wire-Triggered Shock Tube ………………............................ 58

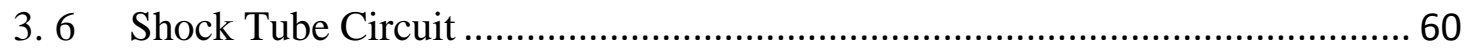

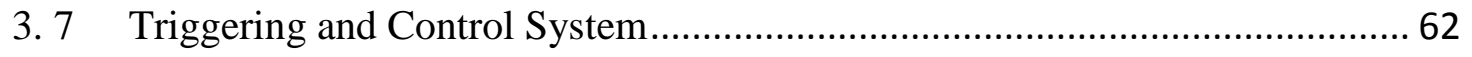

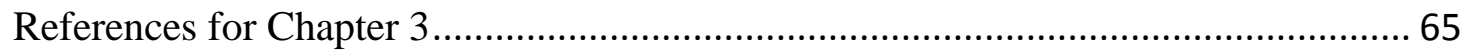

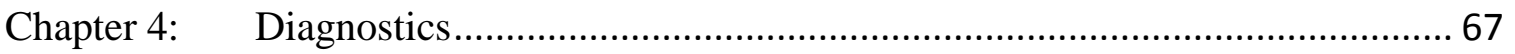

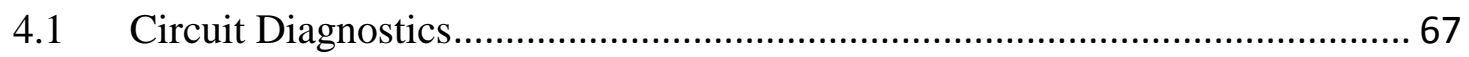

$4.2 \quad$ High Speed Cameras ................................................................................. 73 
4.3 Image Processing Analysis of the TAP ....................................................... 74

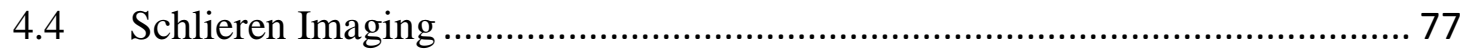

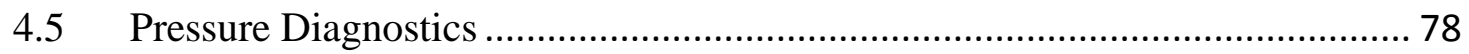

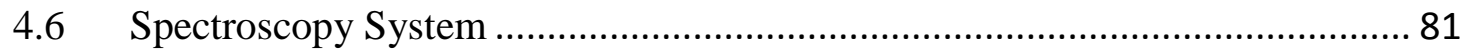

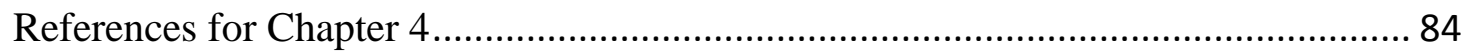

Chapter 5: Toroidal Air Plasma Discharge ............................................................... 85

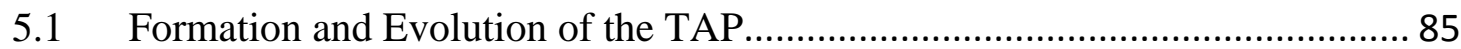

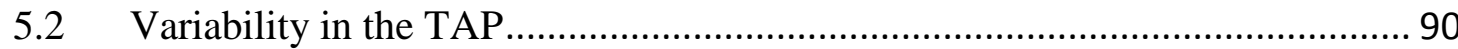

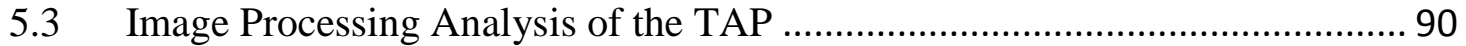

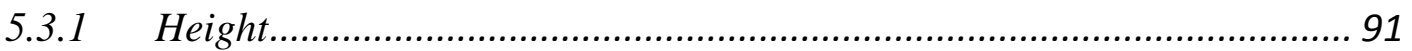

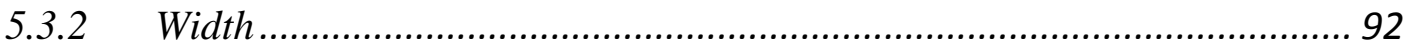

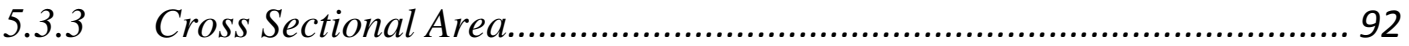

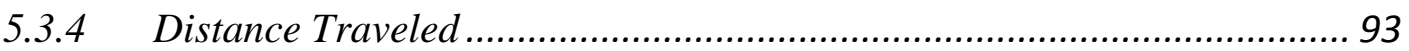

5.3.5 Major Radius and Minor Radius ………………................................. 94

5.4 Interaction Considerations ………..................................................................... 96

5.5 Accelerator Region Investigation ............................................................. 97

5.6 Calculations from the Observed Plasma Current and Voltage Waveforms...... 99 5.6.1 Comparison of Simulated and Observed Waveforms of the Plasma Circuit 99

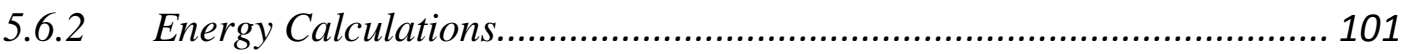

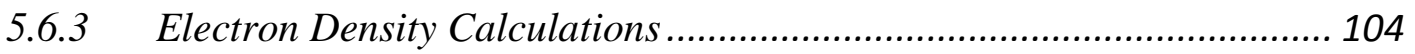

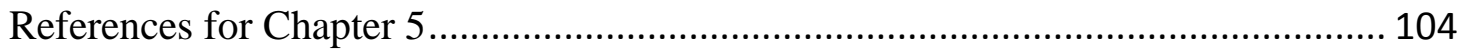

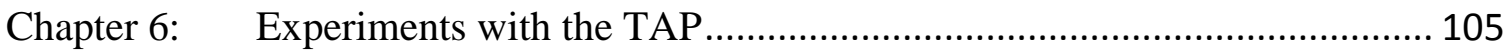

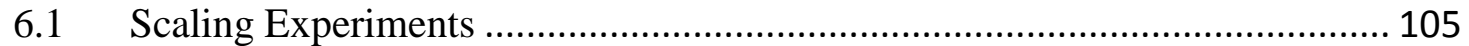

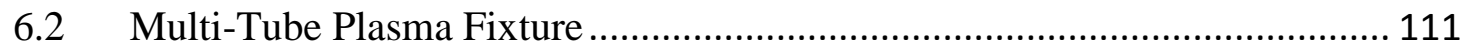

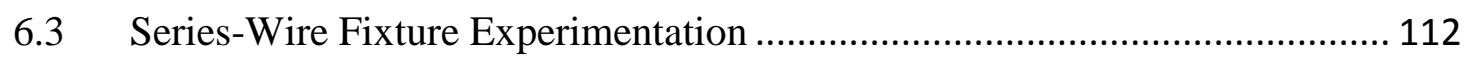

6.4 Energy Scaling Experiments ................................................................... 118

6.5 Varying of the Load and Other Circuit Parameters ...................................... 121

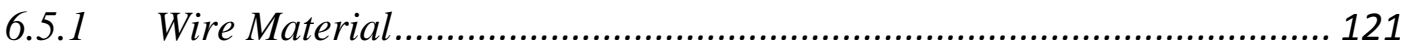

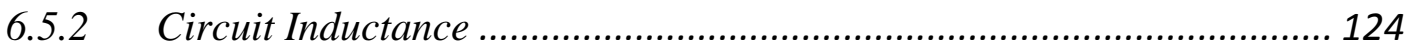

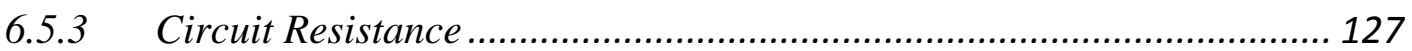

6.6 Magnetic Quadropole Experiments .......................................................... 133

6.7 Measuring the Shock Wave from the Plasma-Generating Exploding Wire ... 137

References for Chapter 6.............................................................................. 140

Chapter 7: $\quad$ Spectroscopy Measurements................................................................ 141

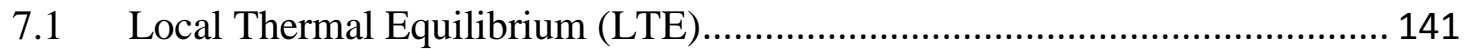

7.2 Spectral Emission of the TAP ..................................................................... 142 


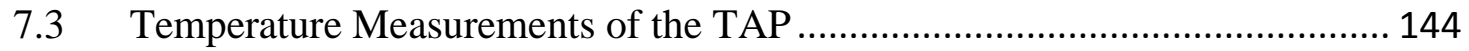

7.4 Stark Broadening Measurements of the TAP ............................................. 148

7.5 Saha-Eggert Calculations of the Electron Density ...................................... 150

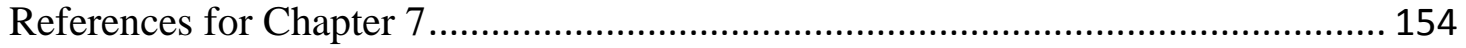

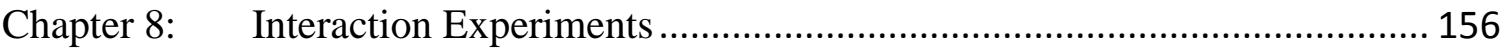

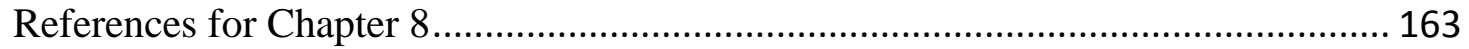

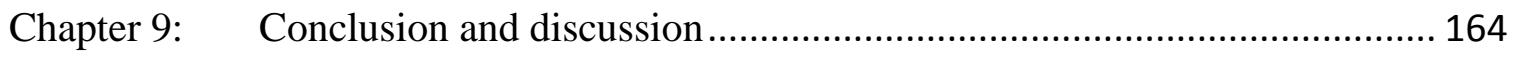

Appendix A: Exploding Wire Simulation............................................................... 169

Appendix B: High Voltage Trigger Circuit and Timing Diagram ............................... 177

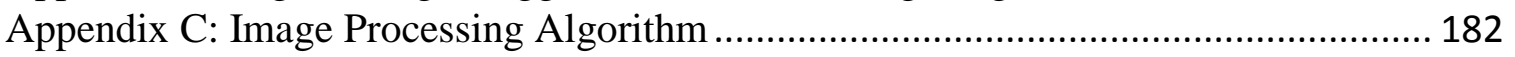

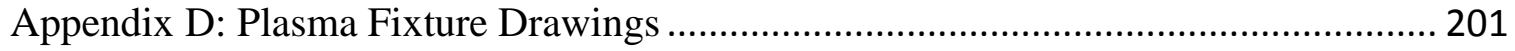

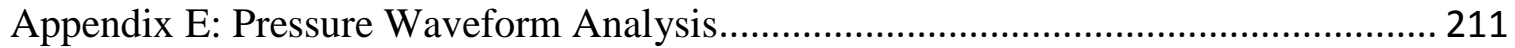

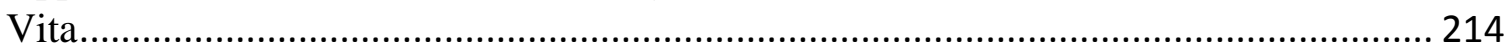




\section{LIST OF FIGURES}

Figure 1.1 Experimental test bench for this research program ...................................... 1

Figure 1.2 Images of the toroidal air plasma .......................................................... 2

Figure 1.3 Shadowgraph images of the flow response to an intensifying plasma spike in a

wind tunnel at Mach 2.5................................................................................ 7

Figure 1.4 Schlieren images of a ballistic sphere flying through a DC discharge plasma 8

Figure 1.5 Experimental diagram of the shock tube experiments in Ganguly et al........... 9

Figure 1.6 Acoustic optical deflection signals in an argon DC plasma ........................... 10

Figure 1.7 Simultaneous measurements of pressure, neutral gas density, luminosity, and

electron density of a shock tube interaction experiment.......................................... 11

Figure 2.1 A typical microhollow cathode geometry .................................................. 22

Figure 2.2 Photograph of a 10 x 10 pyramidal device array discharge .......................... 23

Figure 2.3 Image of typical glow discharge in N2 with UMC MHCD .......................... 23

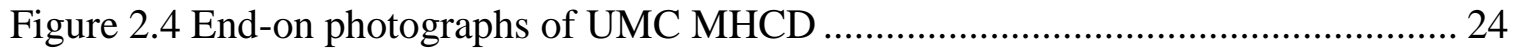

Figure 2.5 UMC cotton threaded fixture................................................................... 25

Figure 2.6 Images of the discharge from the cotton threaded fixture.............................. 26

Figure 2.7 Image of a discharge of an $0.22 \mathrm{~m}$ long cotton threaded fixture .................... 26

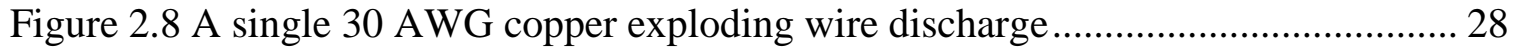

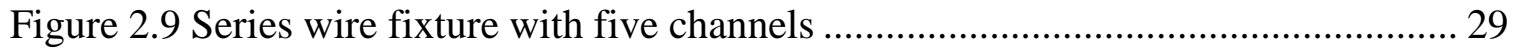

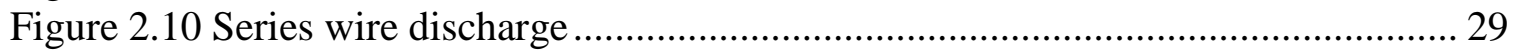

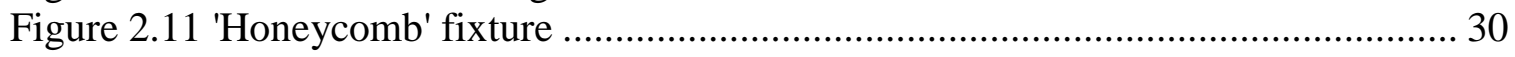

Figure 2.12 Image of the exploding wire discharge from the 'honeycomb' fixture ......... 30

Figure 2.13 Early fixture for the TAP and time-lapsed photograph of the discharge ...... 31

Figure 2.14 Images of the toroidal air plasma from initial experiments ......................... 33

Figure 2.15 Images of the toroidal air plasma labeled with time from vaporization........ 33

Figure 2.16 The 'serpentine fixture' with and without the accelerator screen.................. 34

Figure 2.17 High speed images of the discharge from the 'serpentine' fixture ................. 35

Figure 2.18 Plasma gun with radial magnetic field used to produce plasma rings in

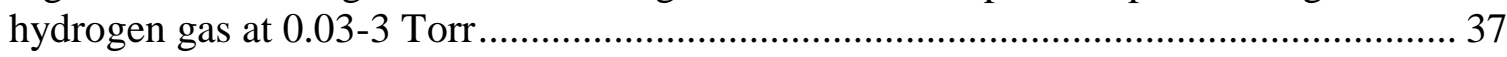

Figure 2.19 Kerr-cell photograph of a magnetized plasma ring ................................... 37

Figure 2.20 Framing camera images of plasma vortex rings in argon gas at 0.1 Torr ..... 38

Figure 2.21 A stable plasma toroid observed in an arc discharge experiment ................ 39

Figure 2.22 Schematic diagram of the plasma injector for capillary discharge............... 40

Figure 2.23 Images of the ejected jet of the capillary discharge. ................................ 41

Figure 3.1 Experimental test bench for this research program ................................. 45

Figure 3.2 Exploding wire driver circuit model......................................................... 46

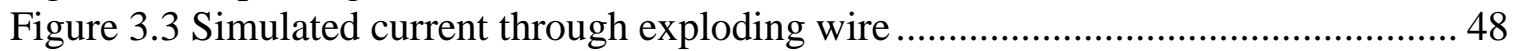

Figure 3.4 Simulated voltage across exploding wire .............................................. 48

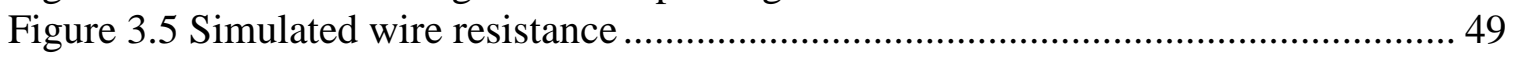

Figure 3.6 Simulated energy deposited into the wire ............................................. 49

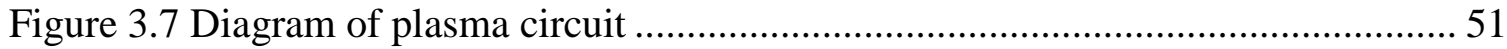

Figure 3.8 Plasma circuit with high voltage capacitor bank .................................... 51

Figure 3.9 Paschen breakdown curve ................................................................... 53 


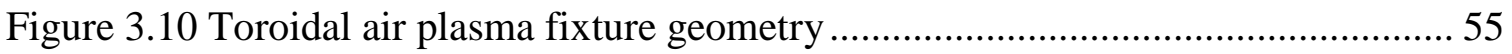

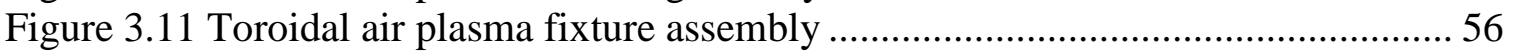

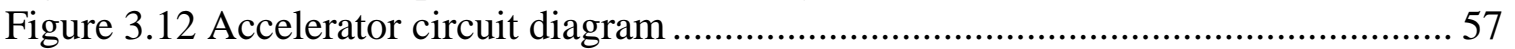

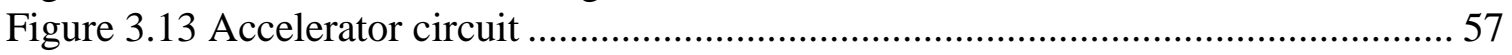

Figure 3.14 Discharge from exploding wire which ruptures the diaphragm ................... 59

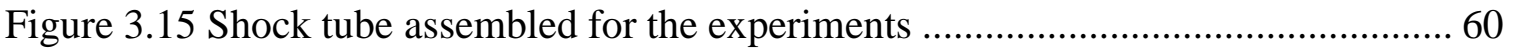

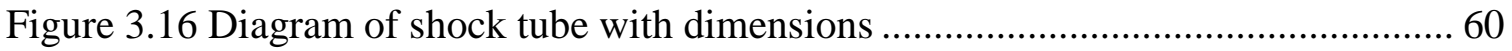

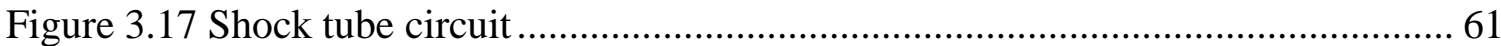

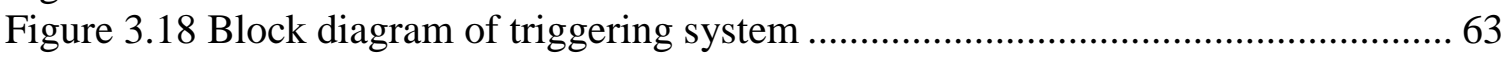

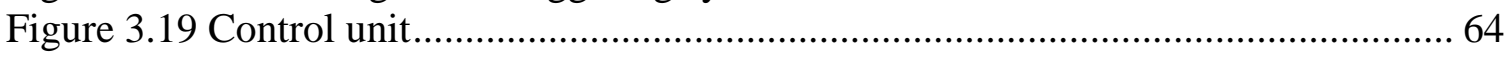

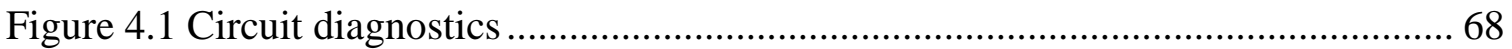

Figure 4.2 Voltage across the exploding wire of the plasma circuit................................ 69

Figure 4.3 Voltage across the exploding wire of the shock tube circuit.......................... 69

Figure 4.4 Voltage divider used to observe the voltage across the accelerator screens ... 70

Figure 4.5 Voltage across accelerator screens ............................................................... 71

Figure 4.6 Current through the exploding wire of the plasma circuit.............................. 72

Figure 4.7 Current through the exploding wire of the shock tube circuit......................... 72

Figure 4.8 Current delivered by the accelerator circuit ................................................... 73

Figure 4.9 High speed cameras at UMC .................................................................... 74

Figure 4.10 Toroid dimensions of the image processing analysis of the TAP ................. 75

Figure 4.11 Schlieren system at UMC ...................................................................... 77

Figure 4.12 Schlieren image of the shock wave .......................................................... 78

Figure 4.13 Pressure waveform of the shock wave ................................................... 79

Figure 4.14 Arrival of shock wave front at the end of the tube ....................................... 80

Figure 4.15 Pressure waveform of the shock wave as measured by an Endevco 8530C-15 mounted on the front planar surface of the plasma fixture.............................................. 81

Figure 5.1 High speed images of the TAP................................................................. 86

Figure 5.2 Toroid dimensions of the image processing analysis of the TAP ................... 87

Figure 5.3 Selected images from Figure 5.1 with details on timing and dimensions of the

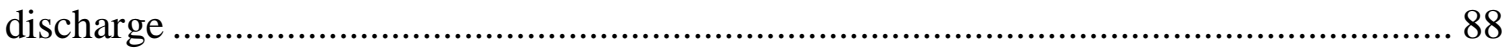

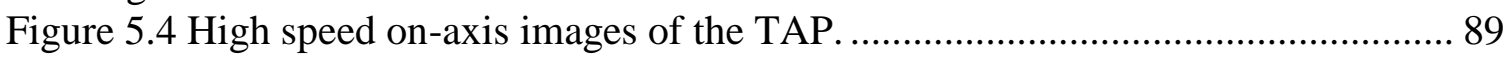

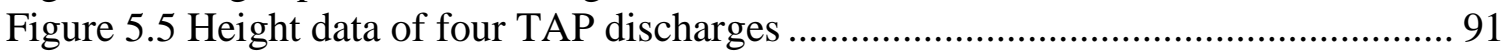

Figure 5.6 Width data of four TAP discharges .............................................................. 92

Figure 5.7 Cross sectional area data of four TAP discharges ......................................... 93

Figure 5.8 Distance traveled data of four TAP discharges .............................................. 94

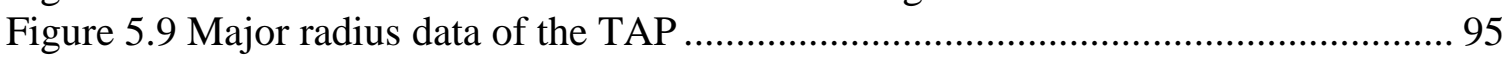

Figure 5.10 Minor radius data of the TAP …………………...................................... 96

Figure 5.11 Area data for the accelerator region investigation experiments .................... 98

Figure 5.12 Distance traveled data of accelerator region investigation experiments ....... 99

Figure 5.13 Comparison of matched simulation and observed current traces of the exploding wire in the plasma generation circuit ............................................................ 100

Figure 5.14 Comparison of matched simulation and observed voltage traces of the exploding wire in the plasma generation circuit 
Figure 5.15 Energy deposited into exploding wire as calculated from voltage and current measurements 102

Figure 5.16 Energy deposited into the plasma discharge from the accelerator circuit as calculated from voltage and current measurements...... 103

Figure 6.1 Alternative length plasma fixtures............................................................... 106 Figure 6.2 High speed images of TAP discharges from the alternative-length experiments 107

Figure 6.3 Comparison of height data of TAP discharges from alternative-length fixtures 108

Figure 6.4 Comparison of width data of TAP discharges from alternative-length fixtures 108

Figure 6.5 Comparison of area data of TAP discharges from alternative-length fixtures

Figure 6.6 Comparison of distance traveled data of TAP discharges from

alternative-length fixtures 109

Figure 6.7 Comparison of the average area of TAP discharges from alternative-length

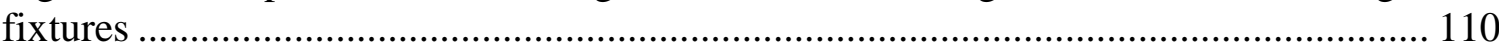

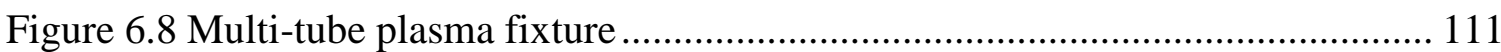

Figure 6.9 On axis images of discharge from the multi-tube plasma fixture ................ 112

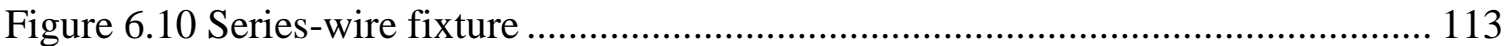

Figure 6.11 Wiring diagram for the series-wire fixture .............................................. 113

Figure 6.12 Image of the toroidal discharge from the series-wire fixture ...................... 114

Figure 6.13 Image of the discharge from the series-wire fixture.................................. 115

Figure 6.14 Comparison of height data of nominal TAP fixture and from the series-wire

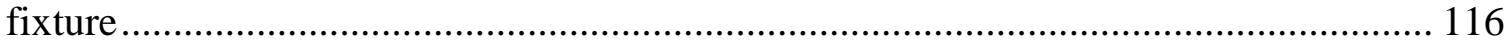

Figure 6.15 Comparison of width data of nominal TAP fixture and from the series-wire

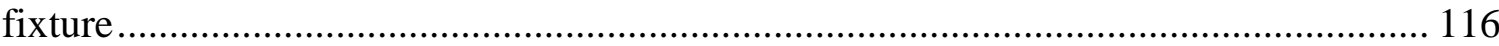

Figure 6.16 Comparison of area data of nominal TAP fixture and from the series-wire

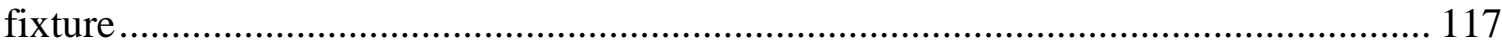

Figure 6.17 Comparison of distance traveled data of nominal TAP fixture and from the

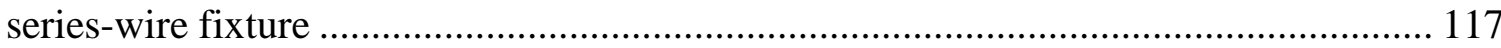
Figure 6.18 Height data of increased energy experiments compared with data from the

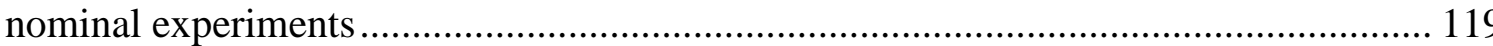

Figure 6.19 Width data of increased energy experiments compared with data from the nominal experiments ............................................................................................ 120

Figure 6.20 Area data of increased energy experiments compared with data from the nominal experiments.

Figure 6.21 Distance traveled data of increased energy experiments compared with data from the nominal experiments ................................................................................ 121 Figure 6.22 Comparison of distance traveled data from different copper wire loads.... 123 Figure 6.23 Comparison of area data from different wire materials.............................. 123 Figure 6.24 Current through the exploding wire for three inductance configurations ... 125 Figure 6.25 Expanded view of the current waveforms of Fig 6.24 .............................. 125 Figure 6.26 Height data of TAP discharges of varying circuit inductance.................... 126 Figure 6.27 Current through the exploding wire for two resistor configurations........... 127 
Figure 6.28 Current through the exploding wire for the circuit: $\mathrm{L}_{1}=11.77 \mu \mathrm{H}$,

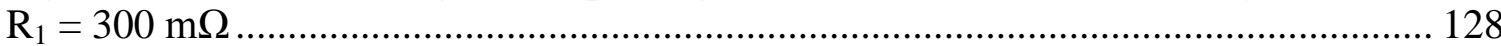

Figure 6.29 Height data of TAP discharges of varying circuit resistance ..................... 129

Figure 6.30 Width data of TAP discharges of varying circuit resistance ..................... 129

Figure 6.31 Area data of TAP discharges of varying circuit resistance ....................... 130

Figure 6.32 Distance traveled data of TAP discharges of varying circuit resistance .... 130

Figure 6.33 High speed images of the TAP $997 \mu$ s from wire vaporization for two resistor

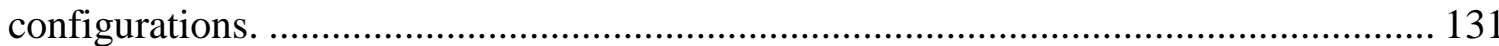

Figure 6.34 Magnetic quadropole fixture, with magnets.......................................... 133

Figure 6.35 Images of the TAP passing through the magnetic quadropole................... 134

Figure 6.36 TAP expanding toward the magnetic fixture from the perspective of the SVSI

Figure 6.37 High speed images of the TAP passing through the magnetic quadropole. 136 Figure 6.38 Diagram of pressure sensor placement for exploding wire shock wave measurements

Figure 6.39 Pressure waveforms of the shock wave from the plasma-generating exploding

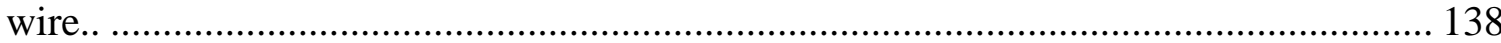

Figure 6.40 Pressure waveforms of the shock wave from the plasma-generating exploding wire (continued data from Figure 6.39) ................................................................. 138

Figure 7.1 Corrected spectral emission of the TAP 143 Figure 7.2 Calibrated average spectrum of the TAP for the initial 200 us after wire vaporization

Figure 7.3 Boltzmann plot of intensity measurements of Fe I transition lines from the TAP discharge. 148

Figure 7.4 Calibrated spectrum of the TAP for the initial 200 us after wire vaporization.

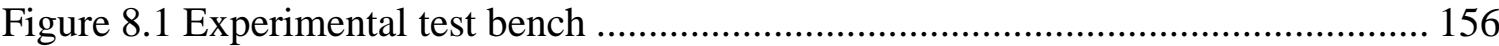

Figure 8.2 High speed images of a shock wave interacting with the TAP .................... 157

Figure 8.3 Schlieren images of a shock wave interacting with the TAP..................... 159

Figure 8.4 Example unprocessed pressure waveforms............................................... 160

Figure 8.5 An expanded view of the data presented in Figure 8.4 ............................. 161 


\section{LIST OF TABLES}

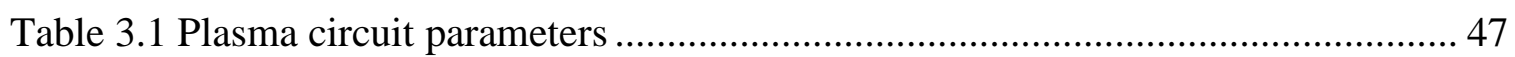

Table 3.2 Exploding wire parameters of the plasma circuit ....................................... 47

Table 6.1 Peak amplitude and time of arrival data of the pressure wave from the plasma circuit exploding wire.

Table 7.1 Wavelength, transition probabilities, lower and upper energy levels, and upper level degeneracy for the Fe I emission lines used for Boltzmann analysis 146 Table 7.2 Wavelength, transition probabilities, lower and upper energy levels, and upper level degeneracy for the Fe II emission lines used for the Saha-Eggert Equation 152 Table 7.3 Electron density calculations of the 44 combinations of Fe I and Fe II emission lines using the Saha-Eggert Equation and the measured intensity of the TAP. 153

Table 8.1 Amplitude reduction of shock wave from interaction with the TAP discharge 162 


\begin{abstract}
The University of Missouri-Columbia has recently developed an experimental test stand for the purposes of studying the interaction of shock waves with plasmas in ambient air. An exploding-wire-triggered shock tube is used to produce the shock wave for interaction with plasma in air.

For the plasma source, an exploding wire discharge has been confined in a cylindrical geometry to form a freely propagating toroid-shaped plasma. The initial plasma is formed by discharging a $11 \mu \mathrm{F}, 4.4 \mathrm{~kJ}$ capacitor bank through a thin wire. This discharge is then forced to expand toward a secondary discharge region which further heats and energizes the plasma. The confining geometry coupled with the secondary heating produces a multi-millisecond duration toroidal air plasma, or TAP. The TAP has a sufficient duration to observe its interaction with the incoming shock wave.

Pressure sensors, high speed photography, Schlieren imagery, emission spectroscopy, as well as current and voltage probes have been configured as a full diagnostic suite to observe the interaction of the shock wave and the TAP discharge. The novel TAP discharge is characterized and explored and the experiments within provide qualitative and quantitative results on the effects of interacting a shock wave with a plasma in ambient air.
\end{abstract}




\section{CHAPTER 1: INTRODUCTION}

This research program at the University of Missouri-Columbia (UMC) was conducted to experimentally investigate the ability of electrically generated plasmas to degrade the amplitude and coherence of a shock wave

Previous academic studies reviewed within have observed mitigation of shock waves as a result of passing through plasma in a variety of low pressure experiments $(4-17.7 \mathrm{kPa})$. The efforts presented are unique in that experiments were conducted in ambient air at nominal atmospheric pressure.

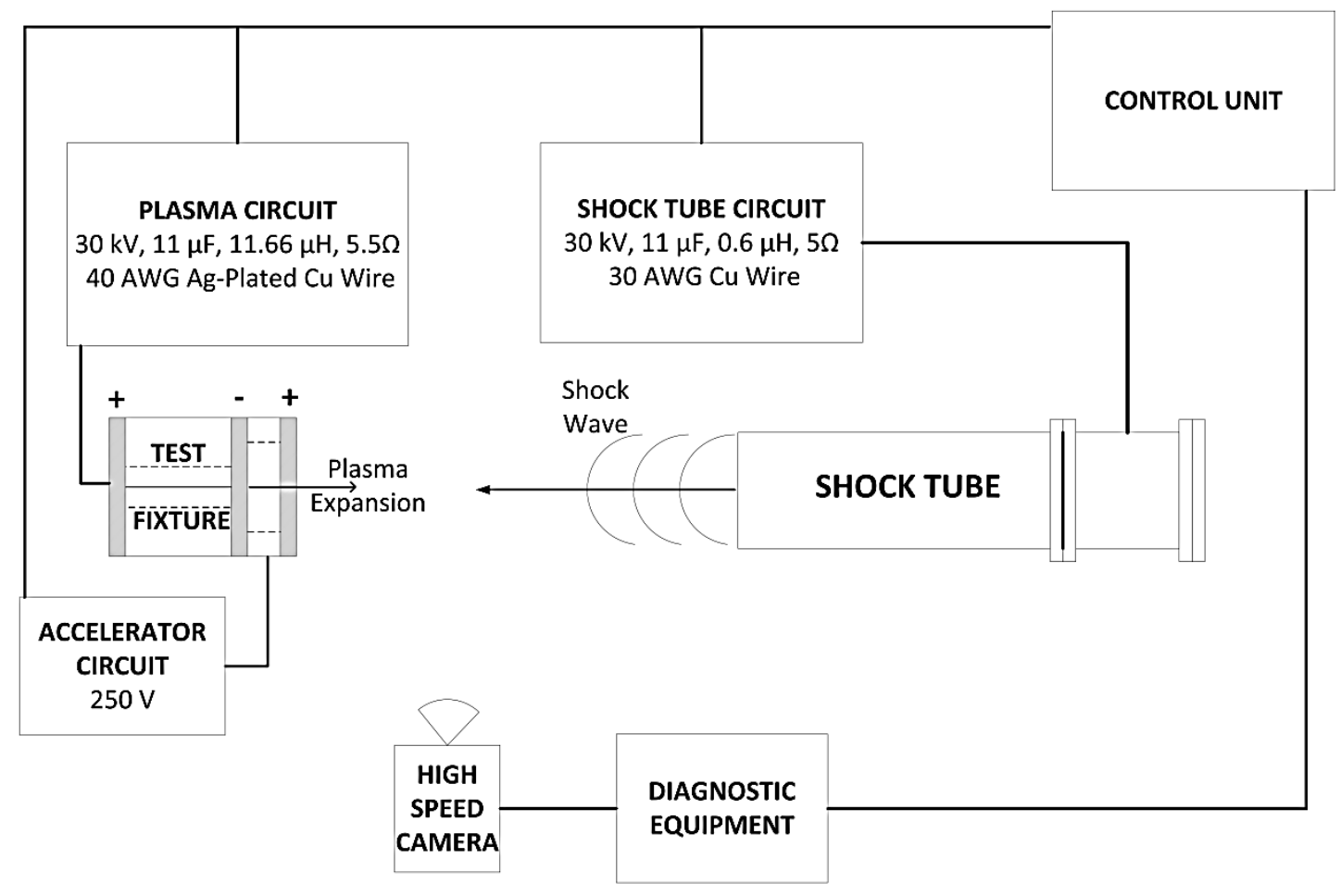

Figure 1.1 Experimental test bench for this research program

An experimental test bench was developed for this research program to investigate the effectiveness of plasma source geometries to mitigate a shock wave produced by a shock tube. The system consists of two pulsed power circuits, an accelerator circuit for imparting more energy into the plasma once it has discharged, an exploding-wire 
triggered shock tube, a central control unit, and a diagnostic suite including high speed cameras. The system is shown as a block diagram in Figure 1.1.
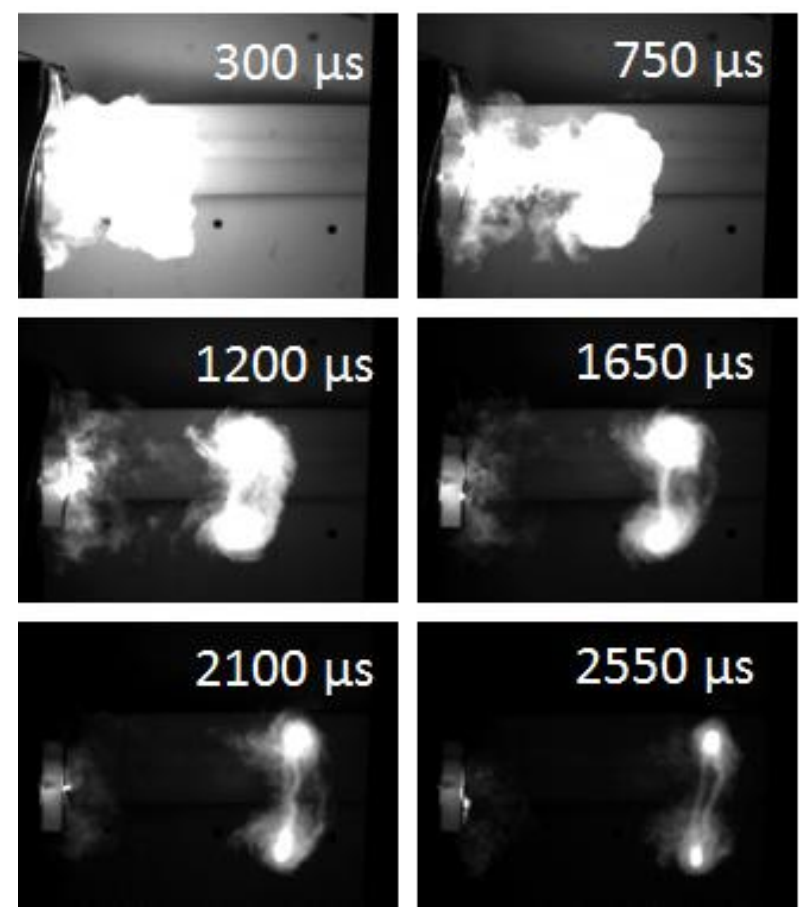

Figure 1.2 Images of the toroidal air plasma labeled with time from vaporization

We have focused on the development and characterization of a viable plasma source for these experiments. A novel, multi-millisecond duration, toroidal air plasma with an electron density of at least $10^{20}-10^{21} \mathrm{~m}^{-3}\left(10^{14}-10^{15} \mathrm{~cm}^{-3}\right)$ has been developed by restricting the radial expansion of an exploding wire. Once an initial plasma is formed, the discharge is subjected to further acceleration from a secondary ci3rcuit, resulting in complex magnetohydrodynamic effects producing a toroidal air plasma, or TAP. The TAP has been observed to be self-sustained beyond $10 \mathrm{~ms}$ while traveling more than $0.25 \mathrm{~m}$, expanding to approximately $0.12 \mathrm{~m}$ in diameter. Typical high speed images of the TAP are shown in Figure 1.2. Through further research, the size of the TAP was more than doubled, creating a discharge which was observed to sustain beyond $20 \mathrm{~ms}$ and travel up to $0.60 \mathrm{~m}$. 
This research program is a significant contribution to the academic community due to the development of the novel toroidal air plasma source, and the results of the interaction experiments. Two pressure sensors observed a reduction in shock wave amplitude as a result of passing through the TAP. The maximum mitigation observed was $50.63 \%$, confirming previously observed effects under low pressure conditions.

The goals of the research program at UMC are summarized in the following itemized objectives:

1. Develop a plasma source with the following minimum specifications:

1.1 Plasma discharge must be produced and sustained in air at atmospheric pressure

1.2 Plasma duration of at least $1 \mathrm{~ms}$

1.3 Peak electron density of $10^{14} \mathrm{~cm}^{-3}\left(10^{20} \mathrm{~m}^{-3}\right)$ or greater

1.4 Plasma discharge must be produced with a minimal timing jitter to facilitate timed interaction with a shock wave

1.5 Plasma discharge must be of significant volume and cross sectional area to facilitate interaction with a shock wave

2. Characterize the following properties of the plasma discharge:

\subsection{Duration}

\subsection{Electron density}

2.3 Temporal evolution of the size and shape of the discharge

3. Develop an experimental apparatus to produce a shock wave with the following properties: 
3.1 Shock wave must be triggered with minimal timing jitter to facilitate timed interaction with the plasma discharge

3.2 Shock wave must be of significant amplitude to measure with traditional pressure diagnostics (i.e. pressure transducers)

4. Integrate the plasma source and shock wave source into one experimental set up for interaction experiments. Interaction experiments must demonstrate:

\subsection{Repeatability}

4.2 The ability to measure the effects of the plasma discharge on the shock wave

5. Design and conduct experiments to optimize the plasma source and investigate scalability of the discharge

This dissertation is a detailed account of the experiments performed at UMC in support of these research goals. To begin this report, previous research into the mitigation of shock waves with electrical discharges is reviewed in the remainder of this chapter. Following a theoretical introduction to plasma physics, an overview of several plasma sources evaluated for this research program are presented in Chapter 2. A detailed overview of the experimental approach and the components of the implemented test bench are discussed in Chapter 3. The diagnostic suite utilized in these experiments is then presented in Chapter 4. The formation, evolution, and characterization of the TAP discharge are presented in detail in Chapter 5. Additional experiments exploring the scalability and optimization of the discharge are presented in Chapter 6. The spectral emission of the TAP discharge is then discussed in Chapter 7. Finally, the interaction experiments are discussed in Chapter 8, and this dissertation concludes with a summary and suggestions for future work in Chapter 9 . 


\subsection{Background on the Interaction of Plasmas and Shock Waves}

The purpose of this research was to study the interaction of shock waves with plasmas in air at atmospheric pressure. Previous studies in a variety of gases (nitrogen, argon, air) and pressures $(4-17.7 \mathrm{kPa})$ indicate that a shock wave traveling through a plasma discharge will decrease in amplitude at the leading edge, spread or split in shock width, and increase in shock velocity. There are three approaches for plasma aerodynamic research: wind tunnel experiments $[1,2]$, ballistic experiments $[3,4]$, and shock tube experiments $[5,6]$.

The physics behind the observed mitigation of the shock wave is not entirely understood or agreed upon by the scientific community. In many cases, researchers have concluded that global heating from the electrical discharge (plasma) can entirely account for the observed mitigation [7-10]. Other studies have observed mitigation of the shock wave beyond that which can be explained by global heating $[3,5,11]$. Bletzinger et al, present a thorough review of a variety of plasma aerodynamic tests along with an outline of previously proposed mechanisms of mitigation in [12].

The following sections will review previous research, and discuss the possible mechanisms of the observed mitigation of shock waves due to interaction with plasma.

\subsubsection{Wind Tunnel Experiments}

Wind tunnel experiments attempt to study the drag of a supersonic object and a bow shock as it moves through plasma. Typical wind tunnel experiments occur in a supersonic wind tunnel with a stationary body. A bow shock wave occurs in front of the object as the supersonic flow passes by the surface. To study the possible mitigation of the shock, plasma is created directly in front of the object on the surface [1,2]. Three main effects 
have been observed in these experiments: the shock standoff distance increases, the drag is reduced, and the heat transfer to the object is reduced.

A study by S. P. Kuo is an example of a plasma mitigation study in a wind tunnel [1]. In this study, an upstream flow with a speed of $570 \mathrm{~m} / \mathrm{s}$, a temperature of $135 \mathrm{~K}$, and a pressure of $17.73 \mathrm{kPa}(0.175 \mathrm{~atm})$ was used in a $0.38 \mathrm{~m}$ x $0.38 \mathrm{~m}$ blowdown wind tunnel. A $60^{\circ}$ cone was used as the model object to generate the flowing shock wave. The tip and the body of the cone object were designed as two electrodes for a gaseous discharge. A $60 \mathrm{~Hz}$ plasma torch was formed by placing a peak voltage of $4.5 \mathrm{kV}$ across the electrodes, resulting in avalanche breakdown. The electric field intensity near the tip exceeded $1 \mathrm{MV} / \mathrm{m}$ before breakdown and reduced to less than $100 \mathrm{kV} / \mathrm{m}$ as the discharge current reached its peak. The peak and average power of the discharge were about $1.2 \mathrm{~kW}$ and $100 \mathrm{~W}$, respectively. The peak electron density of the discharge was estimated to exceed $10^{19} \mathrm{~m}^{-3}\left(10^{13} \mathrm{~cm}^{-3}\right)[1]$.

A continuous 30 frame per second video shadowgraph was utilized to view the flow through the plasma and around the object. The plasma discharge was linearly increased in size and intensity and the modification effect on the bow shock was observed by the video camera. Figure 1.3 shows this in a sequence of four shadowgraphs as the plasma intensifies, where image (a) is the initial condition when the discharge is off. The growth of the plasma discharge is apparent from the increase in background brightness in images (b) - (d). As shown, the shock front moves further upstream, and its shock angle increases. Comparing images (a) and (d) of Figure 1.3, the shock front has transformed from a sharp attached shock into a highly curved shock with a larger shock angle. This is an indication that the shock front is weakened by plasma discharge. 


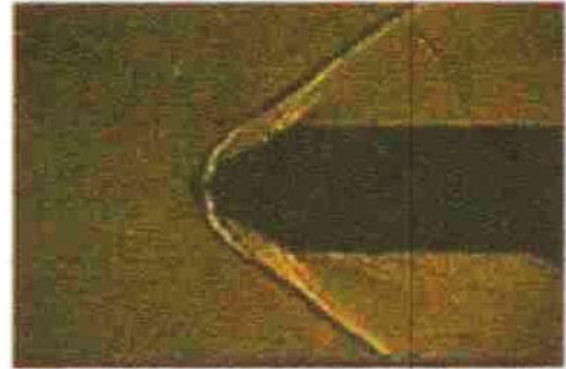

(a)

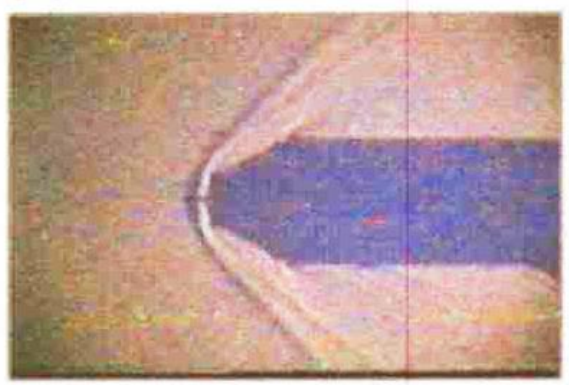

(b)

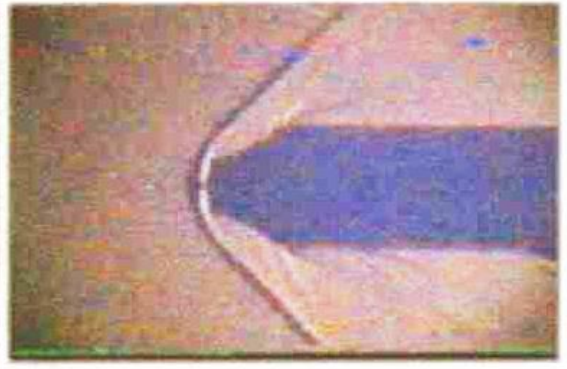

(c)

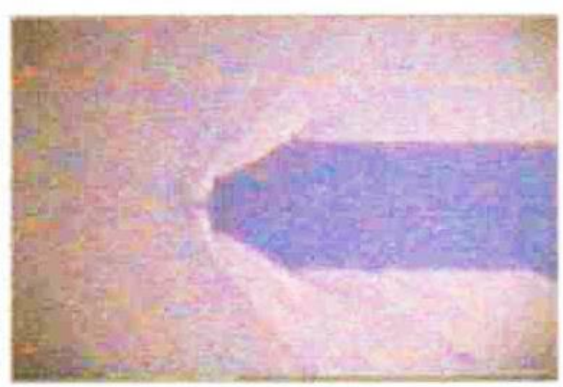

(d)

Figure 1.3 Shadowgraph images of the flow response to an intensifying plasma spike in a wind tunnel at Mach 2.5 [1]

After a thorough analysis, Kuo formulates a theory explaining the deflection of the incoming flow from a symmetrically distributed plasma spike. Kuo concludes that the experimental observations cannot be reasonably explained by heating effects alone [1].

\subsubsection{Ballistic Experiments}

Russian researchers at the Ioffe Physico-Technical Institute have investigated plasma phenomena by launching ballistic projectiles through plasmas and measuring the flow field and drag coefficients [3, 4]. Schlieren photographs, shown in Figure 1.4, from the Ioffe Institute tests show that the shock standoff distance significantly increased for a sphere when flying through a steady DC discharge plasma of electron densities of $10^{17}-10^{18} \mathrm{~m}^{-3}\left(10^{11}-10^{12} \mathrm{~cm}^{-3}\right)$ in gas pressures of $6.66 \mathrm{kPa}$ (50 Torr) [3]. For projectile velocities between $1200-2400 \mathrm{~m} / \mathrm{s}$, the shock standoff distance was observed to increase by a factor of 1.6- 2.2 over comparably heated air [3]. 
Further investigation of ballistic range tests focused on the mitigation effects from thermal nonuniformities, similar to those produced in plasmas $[13,14]$, or on characterizing the decrease in the drag coefficient of a ballistic sphere flying through a plasma [15]. Various groups have found it difficult to reproduce these results due to thermal nonuniformities in the plasma [8-10].

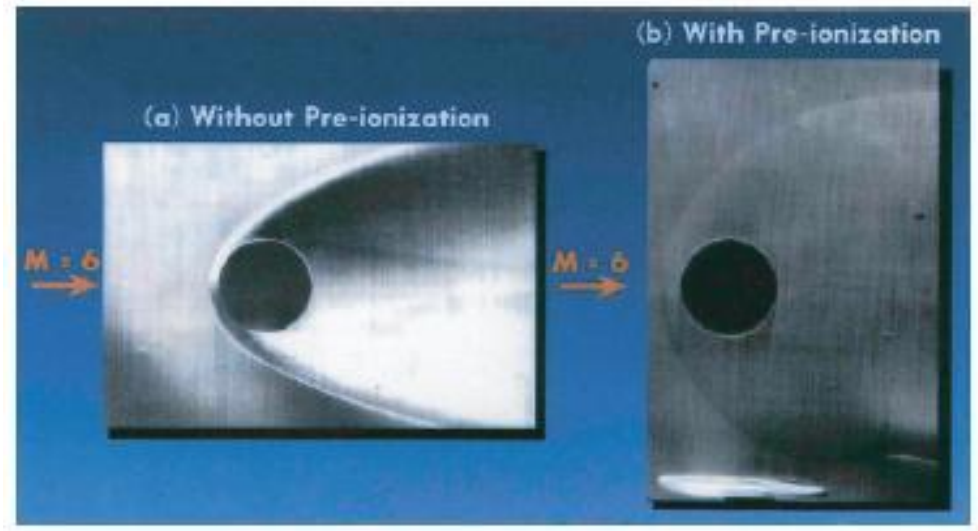

Figure 1.4 Schlieren images of a ballistic sphere flying through a DC discharge plasma [3]

\subsubsection{Shock Tube Experiments}

Shock tube experiments have also shown evidence of shock wave spreading, with decreased front amplitude and increased velocity $[5,6,11,16]$. These experiments involve observing changes to a shock front as it passes through a gas discharge region, all within a gas-filled shock tube. Schlieren photography, pressure transducers, and interferometry are often used simultaneously to characterize the pressure fronts during interaction. The present study conducted at atmospheric pressure has adopted this experimental approach.

Ganguly et al. at the Air Force Research Laboratory, have conducted shock tube experiments with a low pressure, $4 \mathrm{kPa}$ (30 Torr), DC argon plasma inside a $0.05 \mathrm{~m}$ Pyrex tube equipped with a pair of laser deflection diagnostics to measure the characteristics of an acoustic shock wave [5]. The experimental diagram is shown in 
Figure 1.5 [5]. The acoustic wave was generated by the electrical discharge from a spark gap. A regulated, $10 \mathrm{kV}, 300 \mathrm{~mA}$ DC power supply was used to operate the discharge at a constant current from $20 \mathrm{~mA}$ to $140 \mathrm{~mA}$. The acoustic shock propagation was measured with a pair of acoustic laser beam deflection signals recorded on a digital oscilloscope. The typical photo-deflection signals for a Mach 1.8 shock wave are shown in Figure 1.6 with the electrical discharges from $0 \mathrm{~mA}$ (no discharge) to $140 \mathrm{~mA}$ [5].

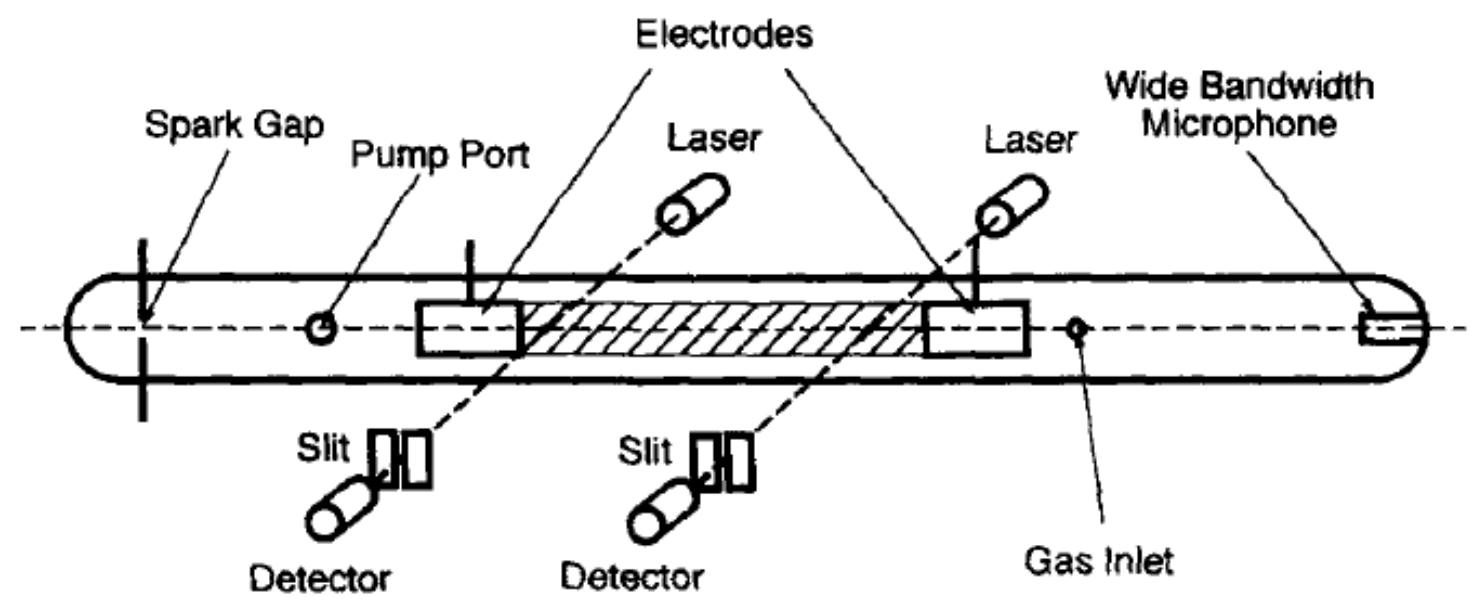

Figure 1.5 Experimental diagram of the shock tube experiments in [5]

As shown, the shock amplitude decreases and spreads in width as the discharge current increases. Comparing the $100 \mathrm{~mA}$ and $140 \mathrm{~mA}$ traces to the $0 \mathrm{~mA}$ and $20 \mathrm{~mA}$ traces, a corresponding increase in shock velocity is observed by the earlier arrival time of the shock wave at the second detector in image (b) of Figure 1.6 [5]. 

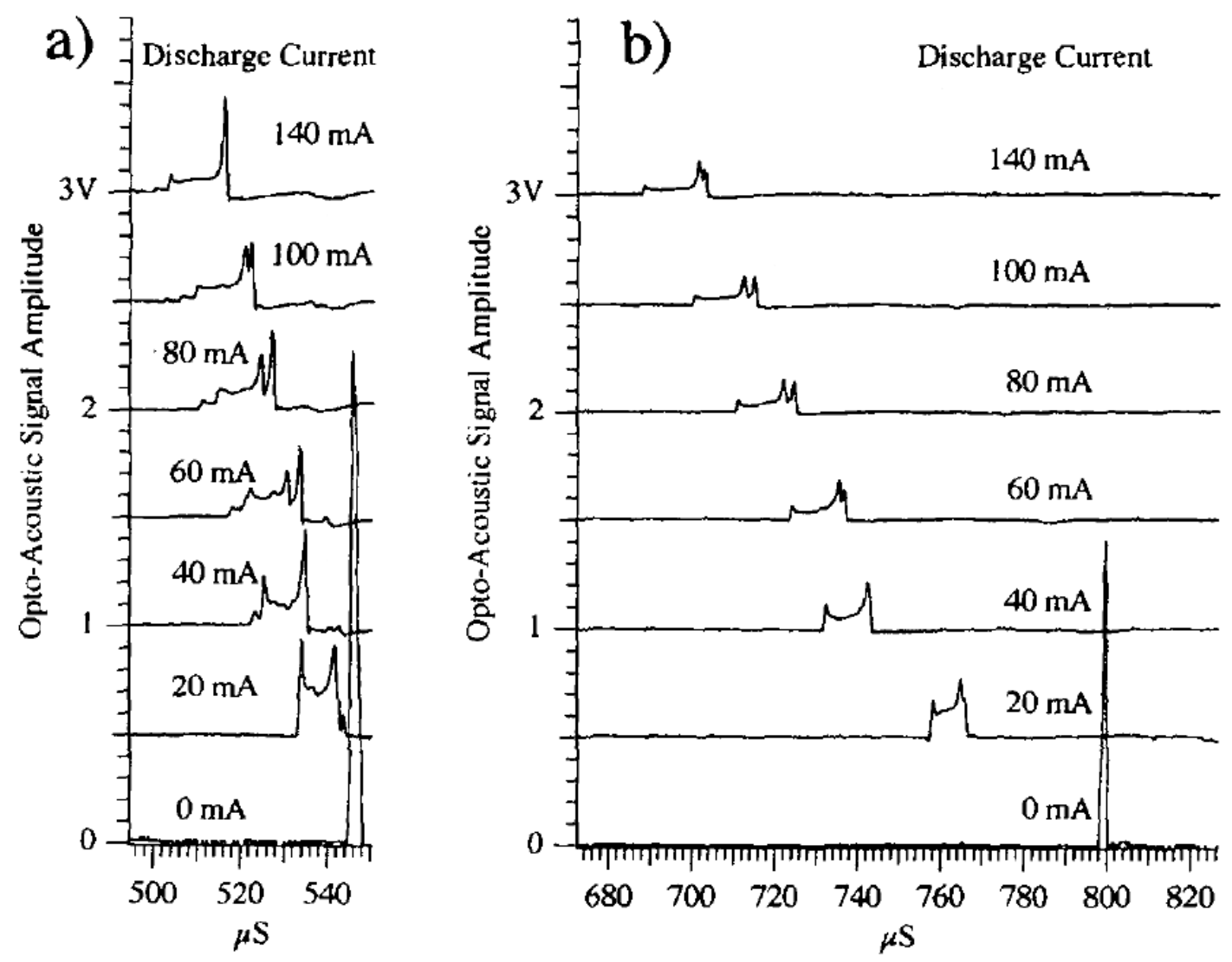

Figure 1.6 Acoustic optical deflection signals in an argon DC plasma taken at a) $0.302 \mathrm{~m}$ and b) $0.422 \mathrm{~m}$ from the shock source [5]

Gorshkov et al studied the interaction of shock waves with a ring discharge in $\mathrm{CO}_{2}$ at $800 \mathrm{~Pa}$ (6 Torr) in a quartz discharge tube [16]. In this study, microwave interferometers measured the electron density at two locations, a laser Schlieren system was used to measure the velocity of the shock wave, and the spatially and temporally resolved plasma luminosity was measured with collimated optics and photomultipliers. Additionally, a moveable pressure transducer was used to further characterize the effects of interaction. Figure 1.7 is a summary of the simultaneous measurements of the pressure, neutral gas density, luminosity, and electron density of this study [16]. The traces for the pressure, P, and the gas density, $\rho$, clearly show a broadening of the shock front. Also, a decrease in 
the luminosity and an increase in the plasma electron density are observed in the A-B region directly before the shock wave front.

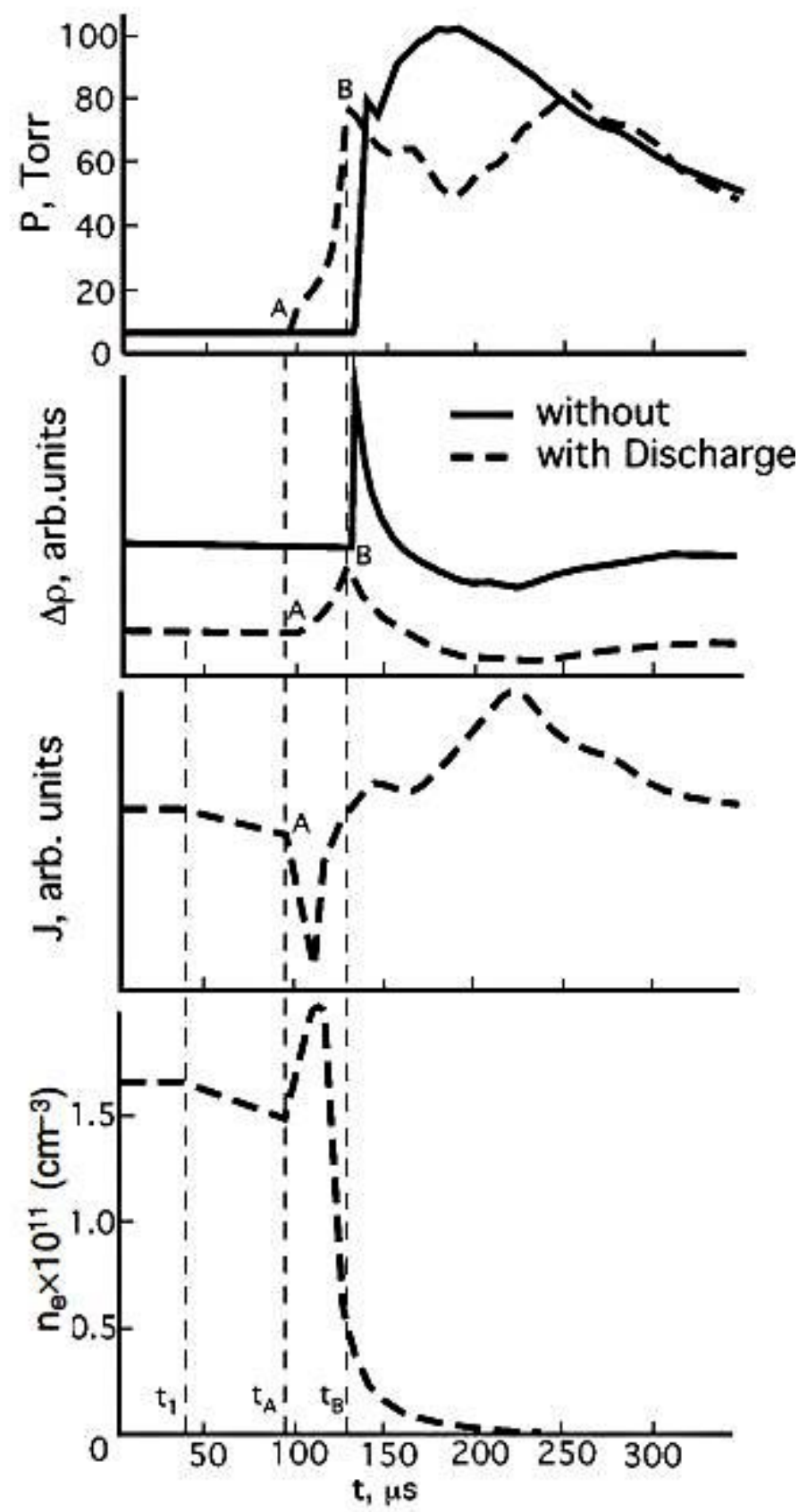

Figure 1.7 Simultaneous measurements of pressure, neutral gas density, luminosity, and electron density of a shock tube interaction experiment [16] 


\subsubsection{Physical Mechanisms of Mitigation}

As discussed, a shock wave is diminished in amplitude, spread or split in width, and increased in velocity when traveling through a plasma. The physical mechanisms behind this mitigation at less than atmospheric pressure are not agreed upon in the scientific community. Thermal nonuniformities present in most plasma make diagnostic techniques difficult. High degrees of precision, accuracy, and reproducibility are necessary to investigate the mechanisms of mitigation. Some mitigation effects can be attributed to the increase in gas temperature due to the plasma [12]. Some experimental results have not observed significant mitigation beyond that which arises from global heating, while other experiments have shown results due to plasma electrodynamic effects $[3,5,7-11,16]$. Bletzinger, et. al present a thorough review of several plasma aerodynamic experiments along with a discussion of the mechanisms involved [12].

A popular approach has been to directly measure the temperature gradient in the plasma as the shock wave propagates through the plasma. This measured temperature is then used in conventional fluid dynamics equations to calculate the expected shock mitigation due to purely thermal effects. In many cases, researchers have calculated that the observed mitigation is higher than that due to global heating $[3,5,11,16]$. Even this approach has arguable inaccuracies due to local thermal nonuniformities that often exist in many gas discharges. Modeling these inaccuracies is highly dependent on precise geometry and boundary conditions.

A combination of computer modeling and empirical results has brought about new explanations for the observed mitigation. When considering experimental data, several researchers have proposed that the shock wave forms a traveling strong double layer 
within the plasma near the leading edge of the shock wave [17-20]. A detailed analysis of shock wave physics in plasmas is given by Zel'dovich and Raizer [21]. As a brief summary of this analysis, the interesting effects associated with a shock wave propagating through an ionized gas are related to the slow energy exchange between ions and electrons and the high electron mobility in a plasma. As a result of these properties, electron heat conduction is much higher than ion heat conduction. When a shock wave propagates through a plasma, the electrons are heated by the shock front. These heated electrons quickly heat neighboring electrons, specifically the ones directly before the shock front, creating a 'pre-heated' front within the plasma directly before the shock wave. While the shock front heats the ions of the plasma in a similar fashion, the ions conduct heat to neighboring ions on a much slower time scale. Thus, in the region of the plasma directly before the shock front the electrons are pre-heated but the ions are not. These conditions are favorable for electron diffusion directly before the shock front, resulting in the creation of space charges, also known as a double layer. If the double layer in the plasma is strong enough with a large potential difference, a significant transfer of energy from the shock front to the gas within the double layer can occur, likely in the form of heating of the vibrational modes of the gas [17-20]. This analysis could explain the mitigation effects previously observed.

Due to practical limitations, a working model for this mechanism is not available at this time, but research has shown correlations between localized increased mean electron energy and increased mitigation effects beyond macroscopic plasma heating [12, 20].

\section{References for Chapter 1:}

1. S. P. Kuo, "On Plasma Mitigation of Shocks," Physical Scripta, vol. 74, pp. 398-404, 2006. 
2. V. P. Gordeev, et al., "Experimental Study of the Possibility of Reducing Supersonic Drag by Employing Plasma Technology," Fluid Dynamics, vol. 31, pp. 313-317, 1996.

3. G.I. Mishin and M.D. Laurel, "Experimental Investigation of the Flight of a Sphere in Weakly Ionized Air,” AIAA, pp.1997-2298, 1997.

4. G.I. Mishin, et al., "Flow Around a Sphere Moving Supersonically in a Gas Discharge Plasma," Sov. Tech. Phys. Lett., vol. 17, pp. 413-416, 1991.

5. B.N. Ganguly, et al., "Shock Wave Damping and Dispersion in Nonequilibrium Low Pressure Argon Plasmas," Phys. Lett. A, vol. 230, pp. 218-222, 1997.

6. A.S. Baryshnikov, et al, "Rearrangement of Shock Waves Structure in a Decaying Discharge Plasma," Tech. Phys. Lett., vol. 23, pp. 259-260, 1997.

7. B. Penetrante and J. Sherohman, "Feasability Study for Analyzing Plasma Aerodynamic Effects," Lawrence Livermore National Laboratory Report, 1999. [online] available: https://e-reports-ext.1lnl.gov/pdf/235538.pdf

8. H. Lowry, et al., "Shock Structure of a Spherical Projectile in Weakly Ionized Air," AIAA, vol. 90, pp. 0600, 1999.

9. H. Lowry, et al., "Ballistic Range Tests in Weakly Ionized Argon," AIAA, vol. 99, pp. 4822, 1999.

10. G. V. Candler, et al., "Vibrational Excitation, Thermal Nonuniformities, and Unsteady Effects on Supersonic Blunt Bodies," 2002, AIAA, vol 40, no 9, pp. 18031810.

11. A.N. Klimov, et al., "Shock Wave Propagation in a Glow Discharge", Sov. Tech. Phys. Lett., vol. 8, pp. 192-194, 1982.

12. P. Bletzinger, et al., "Plasmas in High Speed Aerodynamics," J. Phys. D: Appl. Phys., vol. 38 , pp. R33-R57, 2005.

13. V. P. Goloviznin, G. I. Mishin,Y. L. Serov, I. P. Yavor, Sov. Phys. Tech. Phys., vol. 32, pp. 853-855, 1987

14. Y. L. Serov, I. P. Yavor, Tech. Phys., 199540 248-251

15. A. P. Bedin, G. I. Meshin, Tech. Phys. Lett., 21 5-7

16. V.A. Gorshkov, et al., "Behavior of Electron Density in a Weakly Ionized Nonequilibrium Plasma with a Propagating Shock Wave”, Sov. Phys. Tech. Phys. 32, 1138-1141, 1987.

17. N. Hershkowitz, "Review of Recent Laboratory Double Layer Experiments," Space Sci. Rev., vol 41, pp. 351, 1985.

18. M. A. Raadu, "The Physics of Double Layers and their Role in Astrophysics," Phys. Rep., vol 25., pp. 178, 1989.

19. C. Chan, et al., "Electron Temperature Differences and Double Layers." Phys. Fluids, vol. 26, pp. 1587, 1983.

20. P. Bletzinger, et al., "Electric Field and Plasma Emission Responses in a Low Pressure Positive Column Discharge Exposed to a Low Mach Number Shock Wave," Phys. Plasmas, vol. 7, pp. 4341, 2000.

21. Ya. B. Zel'dovich and Yu. P. Raizer, Physics of Shock Waves and High-Temperature Hydrodynamic Phenomena, London, UK: Academic Press 1967, pp. 515-526. 


\section{CHAPTER 2: PLASMA SOURCE INVESTIGATION}

The primary efforts of this research program focused on developing a plasma source capable of interacting with a shock wave in air at atmospheric pressure. Several sources were initially identified as viable for these experiments. Many of these plasma sources were investigated, designed, and experimentally tested to select one appropriate for the interaction with shock waves. This chapter reviews important concepts of plasma physics, presents a selection of the plasma sources investigated, and comments on each source's candidacy for use in interaction experiments.

\subsection{Review of Plasma Theory}

Plasma is an ionized gas with high internal energy that exhibits certain collective behaviors. As a high-energy collection of charged particles of many species, including electrons, ions, and neutral particles, plasmas are incredibly complex to analyze, especially in the context of this research (air at atmospheric pressure). This section will present a basic review of the equations necessary to discuss and analyze plasmas [1-3].

Plasmas consist of ionized particles in a neutral background gas. Each particle is moving with an individual velocity dependent on electrostatic forces from surrounding particles. This large cloud of interdependent charged particles exhibits certain collective behaviors. Plasmas are considered quasi-neutral, meaning plasmas have an approximately equal number of positive and negative charges. Plasma responds collectively to externally applied fields, and local perturbations can lead to non-local results.

All plasmas must satisfy the general condition that the electrostatic potential energy between the charged particles must be much less than their kinetic energy. If this were not the case, the charged particles would be able to escape, and no grouping or collective 
behavior would occur. This requirement is summarized in the plasma criteria of Equation 2.1 where $n_{o}\left[\mathrm{~m}^{-3}\right]$ is the particle density, and $\lambda_{D}[\mathrm{~m}]$ is Debye length, a plasma parameter which describes the scale on which the charged particles within the plasma screen out electric fields [1].

$$
\begin{gathered}
n_{o} \lambda_{D}^{3} \gg 1 \\
\lambda_{D}=\left(\frac{\varepsilon T_{e}}{e n_{o}}\right)^{1 / 2}
\end{gathered}
$$

The electron Debye length is described in Equation 2.2, where $\varepsilon[\mathrm{F} / \mathrm{m}]$ is the permittivity of the background medium, $T_{e}[\mathrm{eV}]$ is the temperature of the electrons, and $e[\mathrm{C}]$ is the charge of an electron [2]. The Debye length is useful for many contexts, but primarily, it is the characteristic scale length over which the Coulomb potentials of individual charged particles screen out external electric and magnetic fields. Additionally, the Debye length is the distance over which significant charge separation can occur. A Debye sphere, a volume within a radius that is the Debye length, is often used in computational analysis as a spatial resolution parameter.

$$
\Gamma=\frac{1}{4} n \mathrm{v}
$$

Plasmas are often described in terms of particle flux $\Gamma$ shown in Equation 2.3, where $n\left[\mathrm{~m}^{-2}\right]$ is the number of particles and $v[\mathrm{~m} / \mathrm{s}]$ is the velocity [2]. This approach is simple, and is the basis for many simulations involving plasmas where individual particles are tracked and counted through time and space as they interact and collide with each other. However, this approach can quickly become computationally complex as the number of particles increases. Particle flux analysis of air plasmas, such as in this research, requires large amounts of computational resources not available for this project. 
Another analytic method is to treat a plasma as a collection of particles with a statistical distribution of velocity and position. Properties of the average particle, such as average energy and average speed, can then be calculated from statistical analysis. The most common distribution function applied to plasmas is the Maxwell Boltzmann distribution function, the normalized one-dimensional form of which is shown in Equation 2.4 , where $\mathrm{m}[\mathrm{kg}]$ is the particle mass, $\mathrm{T}[\mathrm{K}]$ is the temperature, $v[\mathrm{~m} / \mathrm{s}]$ is the particle velocity, and $\mathrm{k}[\mathrm{J} / \mathrm{K}]$ is Boltzmann's constant [2].

$$
\tilde{f}(v)=\sqrt{\frac{m}{2 k T}} \exp \left[\frac{\frac{1}{2} m v^{2}}{k T}\right]
$$

Analysis of a distribution of charged particles is only useful if the collection fits the distribution. If the collection is not in thermal equilibrium, the distribution will be a poor fit. Fortunately, electrons in plasmas are typically in thermal equilibrium due to their high mobility. If thermal equilibrium, or local thermal equilibrium, can be assumed, then equations such as the Boltzmann Relation, which describes the density of charged particles in an equilibrium state, and Poisson's Equation, which describes the electric potential for a given charge distribution, can be utilized.

The Boltzmann Relation is given in Equation 2.5, where $\varphi[\mathrm{V}]$ is the electrostatic potential within the plasma [2].

$$
n_{e}(x)=n_{o} \exp \left[-\frac{e\left(\phi_{o}-\phi(x)\right)}{k T_{e}}\right]
$$

Poisson's Equation for electrostatics is given in Equation 2.6, where $\rho\left[\mathrm{C} / \mathrm{m}^{3}\right]$ is the charge density [2].

$$
\nabla^{2} \phi=-\frac{\rho}{e}
$$


Another practical method of analysis of plasmas is the evaluation of the conservation of particles, and conservation of momentum within the plasma. The continuity equation using conservation of particles is given in Equation 2.7, where G represents the gains and $\mathrm{L}$ represents the losses of particles attributed to processes such as ionization and recombination [2]. In the case of air plasmas, the losses are expected to be very high as air is a very dense collection of background particles with which the charged particles can collide. An atmospheric pressure air background is a difficult environment in which to produce a long duration high energy plasma discharge, thus requiring a considerable influx of energized charged particles (gains) to facilitate a long, multi-millisecond lifetime.

$$
\frac{\delta n}{\delta t}+\nabla \cdot(n v)=G-L
$$

The conservation of momentum in a plasma is given in Equation 2.8, and it includes the pressure gradient $\nabla_{\mathrm{p}}[\mathrm{Pa} / \mathrm{m}]$, the collision frequency $v_{\text {coll }}[\mathrm{Hz}]$, and Lorentz's Law given in Equation 3.9, which includes the electric field $\mathbf{E}[\mathrm{V} / \mathrm{m}]$, and the magnetic flux B [T] [2].

$$
\begin{aligned}
m n\left[\frac{\delta \mathrm{v}}{\delta t}+(\mathrm{v} \cdot \nabla) \mathrm{v}\right] & =q n[\mathrm{E}+(\mathrm{v} \times \mathrm{B})]-\nabla_{p}-m n v_{c o l l} \mathrm{~V} \\
F & =q(\mathrm{E}+(\mathrm{v} \times \mathrm{B}))
\end{aligned}
$$

When considering specific plasmas with particular geometries and externally applied fields, analysis of the conserved particles and momentum is simplified to a degree, and in many instances, bulk plasmas can be analyzed with more traditional electromagnetic equations such as Maxwell's Equations, given in Equations 2.10 -2.13, where $\mathbf{H}[\mathrm{A} / \mathrm{m}]$ is 
the magnetic field, $\mu_{0}[\mathrm{H} / \mathrm{m}]$ is the permeability of free space, and $\mathbf{J}\left[\mathrm{A} / \mathrm{m}^{2}\right]$ is the current density [2].

$$
\begin{gathered}
\nabla \times \mathrm{E}=-\mu_{o} \frac{\delta \mathrm{H}}{\delta t} \\
\nabla \times \mathrm{H}=\varepsilon_{o} \frac{\delta \mathrm{E}}{\delta t}+\mathrm{J} \\
\varepsilon_{o} \nabla \cdot \mathrm{E}=\rho \\
\nabla \cdot \mathrm{H}=0
\end{gathered}
$$

Plasmas are also often analyzed as fluids using the Vlasov equation, shown in Equation 2.14 , where the plasma is described by a function $f$ of time-varying threedimensional position, three-dimensional velocity, and including the velocity $\mathbf{v}[\mathrm{m} / \mathrm{s}]$, the positional gradient $\nabla_{\mathrm{r}}$, the acceleration $\mathbf{a}\left[\mathrm{m} / \mathrm{s}^{2}\right]$, and the velocity gradient $\nabla_{\mathrm{v}}[\mathrm{m} / \mathrm{s}][2]$.

$$
\frac{\delta f}{\delta t}+\mathbf{v} \cdot \nabla_{r} f+\boldsymbol{a} \cdot \nabla_{v} f=0
$$

Another means of analyzing plasmas is through magnetohydrodynamics, or MHD. The ideal MHD equations are a combination of the Navier-Stokes equations of fluid dynamics and Maxwell's Equations [4, 5]. Magnetohydrodynamics is a macroscopic theory, and can be used to describe a plasma as a bulk conductive fluid. The first ideal MHD equation, shown in Equation 2.15, is the continuity equation for a fluid, a form of Equation 2.7 [5]. In the following MHD equations, $\rho\left[\mathrm{kg} / \mathrm{m}^{3}\right]$ is the mass density, $\mathbf{v}[\mathrm{m} / \mathrm{s}]$ is the bulk velocity, and $p[\mathrm{~Pa}]$ is the pressure of the fluid.

$$
\frac{\delta \rho}{\delta t}+\nabla \cdot \rho \mathbf{v}=0
$$


The second MHD equation, shown in Equation 2.16, is the equation of motion and is equivalent to the conservation of momentum in Equation 2.8 [5].

$$
\rho\left(\frac{\delta \mathbf{v}}{\delta t}+(\mathbf{v} \cdot \nabla) \mathbf{v}\right)=-\nabla p+\frac{1}{\mu}(\nabla \times \mathbf{B}) \times \mathbf{B}
$$

Equation 2.17 is the equation of energy for a fluid written in terms of pressure $p$ [Pa] [5].

$$
\frac{\delta p}{\delta t}=-\mathbf{v} \cdot \nabla p-\Gamma p \nabla \cdot \mathbf{v}
$$

The final MHD equation, Equation 2.18, is a form of Faraday's Law [5].

$$
\frac{\delta \mathrm{B}}{\delta t}=\nabla \times(\mathrm{v} \times \mathrm{B})
$$

No matter the method of analysis, plasmas are described by numerous partial differential equations. The large volume, high pressure, long duration plasma sources of this study are very complex and difficult to analyze even through sophisticated analytic and numeric methods. I would argue that the plasma is decaying and it may not be in thermodynamic equilibrium. Given these issues, we focused on experimental characterization during our plasma source investigation, the data from which can be used in future work to scale and improve the chosen plasma source with such numerical analysis.

\subsection{Plasma Source Investigation}

A limited number of known plasma sources are stable at atmospheric pressure. A detailed account of the current state of research in atmospheric-pressure plasmas is given by Kunhardt and Schütze et. al. in [6, 7]. Several plasma sources were identified as possible candidates for this research, such as microhollow cathode discharges and exploding wires. Other plasma sources were not investigated due to low charge density (RF dielectric barrier discharge [8,9]) or high cost (laser produced plasmas [10, 11]). 
Additional novel plasma sources were designed, fabricated, and tested for this research program. This section is a description of a selection of the plasma sources investigated, along with discussion of the viability of each plasma source as a candidate for the interaction with shock waves.

\subsubsection{Microhollow Cathode Discharge}

Hollow cathode discharges are gas discharges between an arbitrary anode and a cathode with a hollow, typically cylindrical, hole. This geometry allows for three distinct modes of current-voltage operation for the gas discharge at pressures such that the product of pressure multiplied by the cathode hole diameter, $p D$, is on the order of Torr$\mathrm{cm}$. At low currents, a glow discharge forms with the shape of the electric field, extending from the cathode to the anode through the cathode hole $[12,13]$.

With increasing current, the plasma column in the cathode hole changes the electric field distribution from axial to radial, accelerating electrons generated at the cathode toward the axis. In certain $p D$ conditions, these electrons that are accelerated toward the axis reach the opposite cathode and are accelerated back toward the axis. This allows for an oscillation with diminishing amplitude of electrons within the column between the two cathode fall regions. The increased ionization rate of these oscillating electrons in the hollow cathode results in a decrease in voltage with an increase in current (negative differential resistivity). This discharge is known as the hollow cathode discharge $[12,13]$.

With a further increase in current, the discharge expands over the surface of the cathode above the hole, eventually transitioning to a glow. This is characterized by a constant current with increasing discharge voltage. When the discharge reaches beyond the boundaries of the cathode, increased voltage results in increased current (positive 
differential resistivity), and the discharge is known as an abnormal glow discharge $[12,13]$.

Scaling using similarity laws, suggests that hollow cathode discharges can operate at or near atmospheric pressures $[12,13]$. Further explanation of the physics of hollow cathode discharges can be found in [12-15]. A common geometry for hollow cathode discharges is shown in Figure 2.1. Cathode holes on the order of $100 \mu \mathrm{m}$ in diameter have been shown to produce discharges at atmospheric pressure $[12,16]$. Discharges in this geometry at atmospheric pressures are typically referred to as microhollow cathode discharges, or MHCD $[12,13]$. The MHCD geometry is used in several studies on hollow cathode discharges $[12,13,17-20]$.

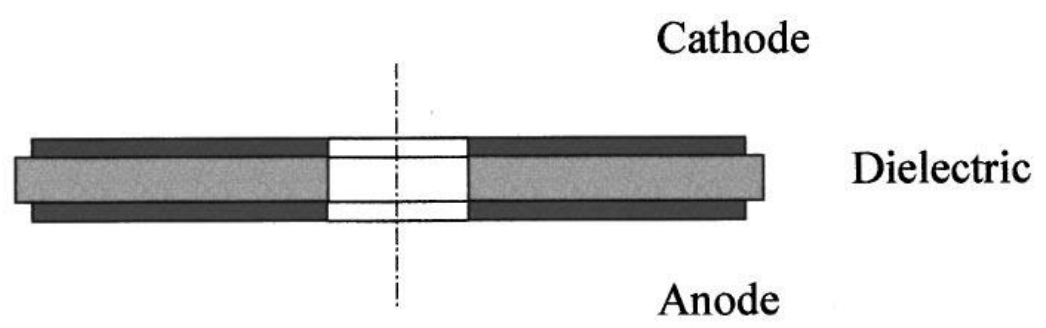

Figure 2.1 A typical microhollow cathode geometry [16]

A MHCD source is an attractive option for the interaction with shock waves, as previous studies have produced discharges in air [21, 22-25], and other studies have observed electron densities of these devices of at least $10^{21} \mathrm{~m}^{-3}[12,26]$.

Additionally, many previous studies focused on methods of creating large volume atmospheric glow plasmas utilizing MHCD. The principal advantage of the MHCD is the ability to produce an abnormal glow discharge. The positive differential resistivity of the abnormal glow discharge allows for the combination of several MHCD's in parallel without the use of resistive ballasting. Without resistive ballasting, arrays of MHCD's are easily manufactured for the purpose of operating in parallel to create larger volume 
plasma. Several studies have investigated parallel arrays of microdischarge devices, like the one shown in Figure 2.2 [12, 21, 27-34].

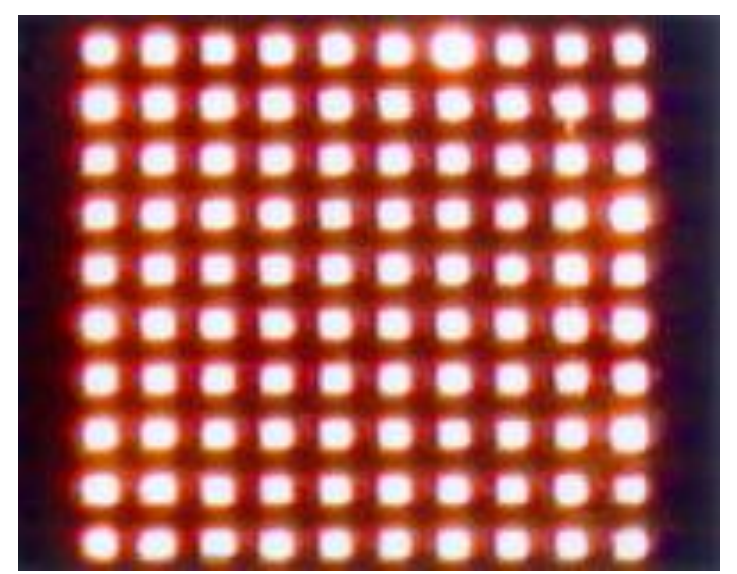

Figure 2.2 Photograph of a $10 \times 10$ pyramidal device array discharge, $244 \mathrm{~V}, 20 \mathrm{~mA}, 1200$ Torr in neon [29]

The microhollow cathode geometry shown in Figure 2.1 was replicated to support the research efforts at UMC with $\mathrm{Cu}$ electrodes and an $\mathrm{Al}_{2} \mathrm{O}_{3}$ dielectric. An array of $125 \mu \mathrm{m}$ microhollow holes were laser drilled for the purpose of creating a large area atmospheric pressure glow discharge plasma. A simple circuit with a series resistor and a current sensing resistor was fabricated. A vacuum chamber containing the microhollow discharge device is shown in Figure 2.3. The system provides gas flow through the holes during normal operation. The system also allows for a variety of diagnostics, including voltage and current measurements, spectral emission analysis, and a high speed camera to observe the development of the glow discharge.

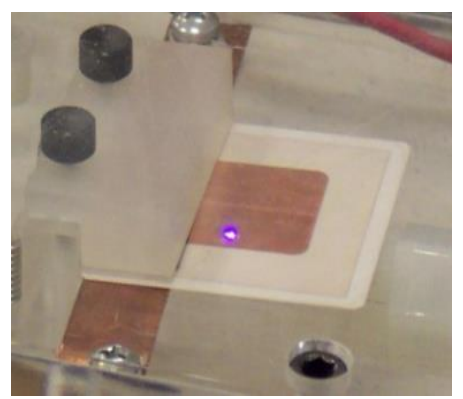

Figure 2.3 Image of typical glow discharge in N2 with UMC MHCD. $630 \mathrm{~V}, 3.4 \mathrm{~mA}, 250$ Torr 
Stable DC glow discharges have been observed at pressures up to $93 \mathrm{kPa}$ (700 Torr) in flowing $\mathrm{N}_{2}$. Photographs of the discharge are shown above in Figure 2.3 and in Figure 2.4. Typical stable single discharges first formed around $600 \mathrm{~V}$ at a current of $1 \mathrm{~mA}$. Increasing the current to $6 \mathrm{~mA}$ expands the radius of the discharge to about $2.2 * 10^{-3} \mathrm{~m}$, encompassing the area of several holes. However, this does not form discharges in other holes of the array. Thus, parallel discharge operation was not achieved. The electron density of this discharge is estimated to be $10^{14} \mathrm{~m}^{-3}$ using the method outlined in $[22,25]$. While this calculation is fairly crude, the present study is several orders of magnitude lower than previous studies $[12,26]$.

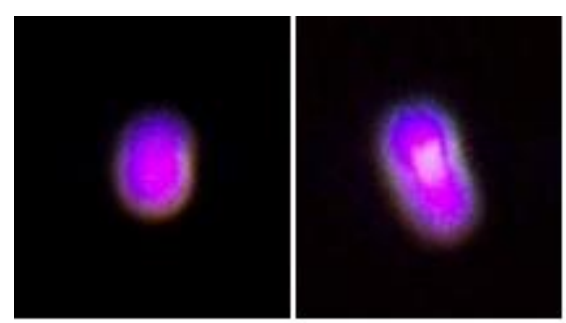

Figure 2.4 End-on photographs of UMC MHCD, diameter $\sim 2.2 * 10^{-3} \mathrm{~m}, 630 \mathrm{~V}, 3.4 \mathrm{~mA}$, $33.3 \mathrm{kPa}$

Increasing the pressure in the chamber decreased the size of the glow discharge above the cathode. Beyond $53 \mathrm{kPa}$ (400 Torr), instabilities in the discharge were observed. These instabilities included flickering in the emission and sharp spikes in the voltage across the electrodes. However, a stable discharge was present up to $93 \mathrm{kPa}$ (700 Torr) for several seconds at a time between instabilities. Additional testing in argon at similar pressures showed comparable results to that of nitrogen. Atmospheric air discharges were observed and deemed unstable.

It is believed that these exact experimental conditions did not allow for stable glow discharges in air at atmospheric pressure. The copper electrodes appear to have been a poor electrode material due to localized heating, which lead to ablation of the surface. A 
variety of alternate experimental conditions were considered such as molybdenum electrodes and alternate flow gases. Pulsed operation of these discharges could also enhance the peak power delivered to the microhollow cathode device, allowing for improved heat management and possible parallel operation.

More information on the conducted MHCD experiments was published in [35]. Due to the small volume and instabilities of the discharges, further investigation of a viable large area plasma source from an array of MHCD was tabled in favor of investigating other plasma sources.

\subsubsection{Cotton Threaded Discharge}

Experiments with electrical discharges formed along cotton threads were conducted to find a viable atmospheric air plasma source for the interaction with shock waves. In several geometries and configurations, an anode-cathode gap was connected by a thread. The length of the thread (and thus the anode-cathode gap) was varied and multiple thread materials were tested to explore the largest and longest-duration discharge. Parallel discharges along several threads were not achieved.

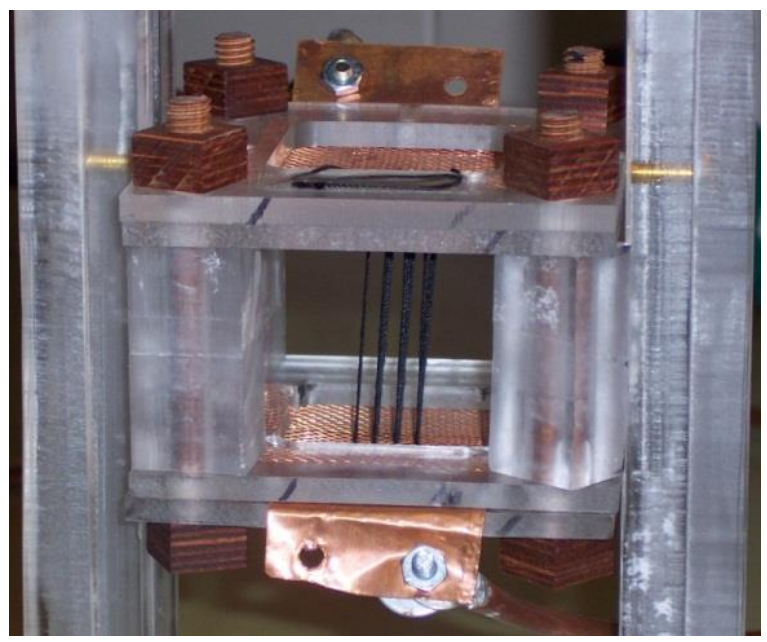

Figure 2.5 UMC cotton threaded fixture 
The typical configuration of the fixture is shown in Figure 2.5, with a black mercerized cotton thread of thickness $3.175^{*} 10^{-4} \mathrm{~m}$. This thread was soaked in $5 \% \mathrm{NaCl}-$ $\mathrm{H}_{2} \mathrm{O}$ solution then dried to coat the thread in salt to make an electrically conductive thread. An alternate material which also produced a similar discharge is an aluminized flash braided thread.
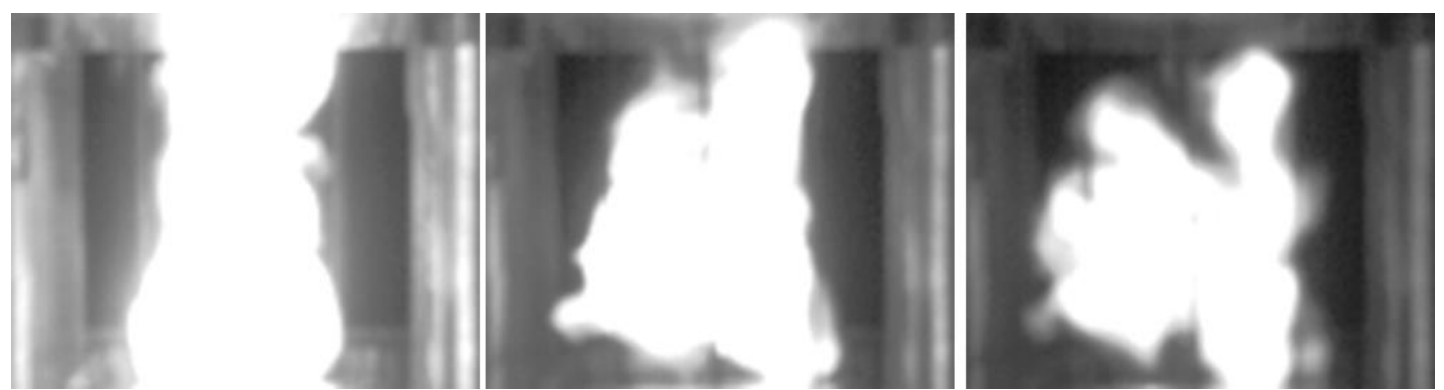

Figure 2.6 Images of the discharge from the cotton threaded fixture, images are $1 \mathrm{~ms}$ apart

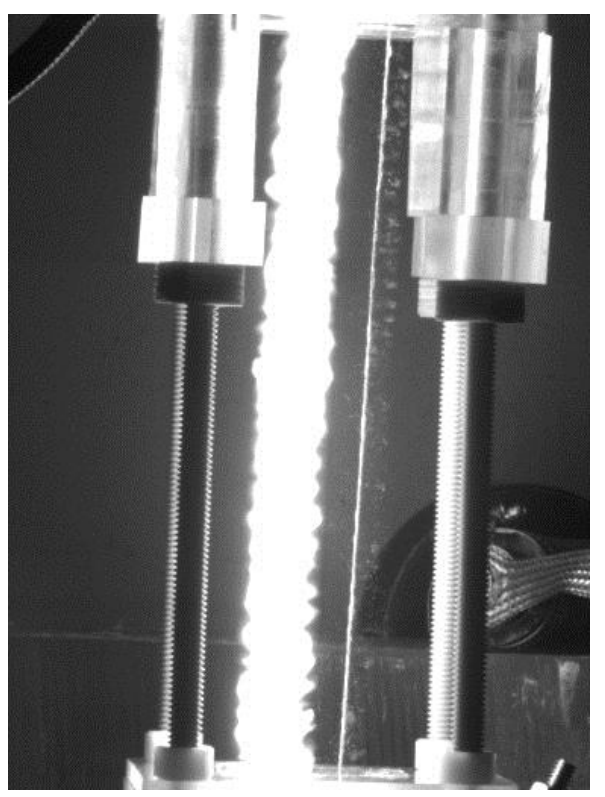

Figure 2.7 Image of a discharge of an $0.22 \mathrm{~m}$ long cotton threaded fixture

The fixture allows for a $0.5 \mathrm{~m} \times 0.5 \mathrm{~m}$ open area for the plasma to form. Figure 2.6 is a series of images from a high speed camera of a typical discharge from the NaCl-cotton thread. These images were recorded with a $1 \mathrm{~ms}$ period. Typical discharges emit light for 8-10 ms and fill a peak volume of roughly $1.9 * 10^{-5} \mathrm{~m}^{3}$. Experimentation with the length of the fixture concluded that the discharge occurred with elongated fixtures of up to at 
least $0.22 \mathrm{~m}$ in length, a discharge of which is shown in Figure 2.7. This discharge was identified as a viable candidate for interaction with shock waves due to its relatively minimal geometry and long duration.

\subsubsection{Exploding Wire Plasma Sources}

Previous research has involved the use of exploding wires as an opening switch [36]. Due to this expertise, a previously-developed pulsed power circuit was used to investigate several experimental exploding wire plasma sources. Ultimately, this circuit was used throughout this research program and is presented in detail in Chapter 3 as well as in its original form in [36].

Driving a thin wire with a large, fast-rising current is a well-known, well-studied phenomenon [36- 40]. A fast rise in current through a thin wire leads to rapid heating and state transition. As the wire temperature increases, a dramatic decrease in resistivity occurs and additional current is able to flow through the wire. The additional current, and further drop in resistivity, causes the wire to rapidly and violently transition from a solid to a gas state. This violent explosion can lead to a plasma similar to an arc discharge. The initial period of low current is known as the current pause or dwell time, and the following high current renewal is termed restrike. The common explanation for restrike is that the electric field exceeds the dielectric strength of the fuse wires and an arc breakdown occurs. The medium around the fuse, known as the quenching or tamping material, is used to slow the expansion of the fuse and delay the reduction in dielectric strength [36-38].

Several models of the mechanisms of wire explosion were proposed during conferences on exploding wire research in the 1950s and 1960s [37, 38]. Equations of 
state and MHD models have been developed to describe the behavior and physical phenomena of wire explosions $[39,40]$. Additional models have been developed to describe the dynamic resistivity of an exploding wire as an interdependent component of the driving circuitry $[36,41,42]$. Some critical parameters in modeling exploding wires include the energy dissipated in the wire, and the action integral, given in Equation 2.19 where $h$ is the current action, and $i[\mathrm{~A}]$ is the current [41]. These factors are dependent on circuit parameters and material properties of the wire. Additionally, the medium surrounding the wire plays a critical role in the expansion of the discharge and the energy dissipated into the wire [38].

$$
h(t)=\int_{0}^{t_{0}} i^{2}(t) d t
$$

For this research program, several experimental exploding wire plasma sources were investigated. Early trials were conducted with a single wire exploding in ambient air, shown in Figure 2.8. A single 30 AWG wire explodes and forms a discharge with a duration of approximately $400 \mu \mathrm{s}$. Several fixtures, some of which are reviewed below, were designed and fabricated to explode multiple wires in constrictive geometries in attempts to increase the duration of the discharge.

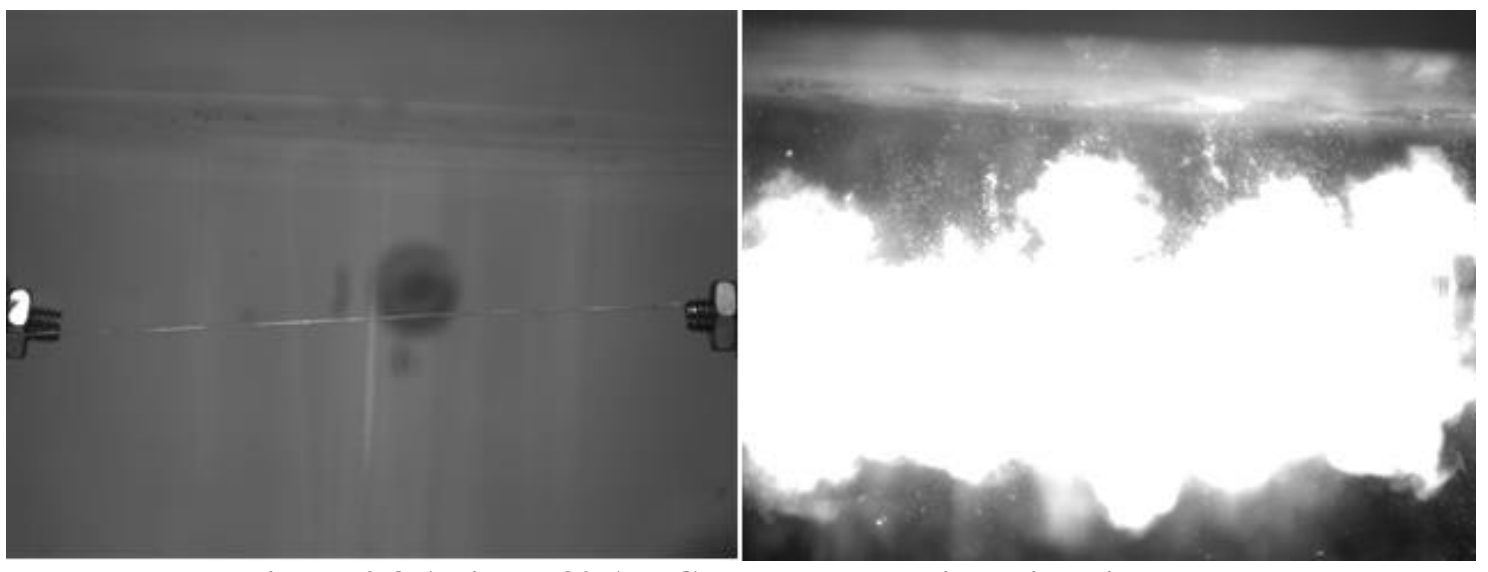

Figure 2.8 A single 30 AWG copper exploding wire discharge 
One configuration tested was a long series wire in five channels. The fixture is shown in Figure 2.9. The five sections of wire explode in succession, with the bottom igniting first, approximately $30 \mu \mathrm{s}$ before the top wire. Plasma discharges form within each channel, with an observed duration of over $1 \mathrm{~ms}$, with a significant decrease in luminosity after $550 \mu \mathrm{s}$. An image of the discharge $100 \mu \mathrm{s}$ after vaporization is shown in Figure 2.10, where the bottom discharge has mostly dissipated while the other channels are still luminescent.

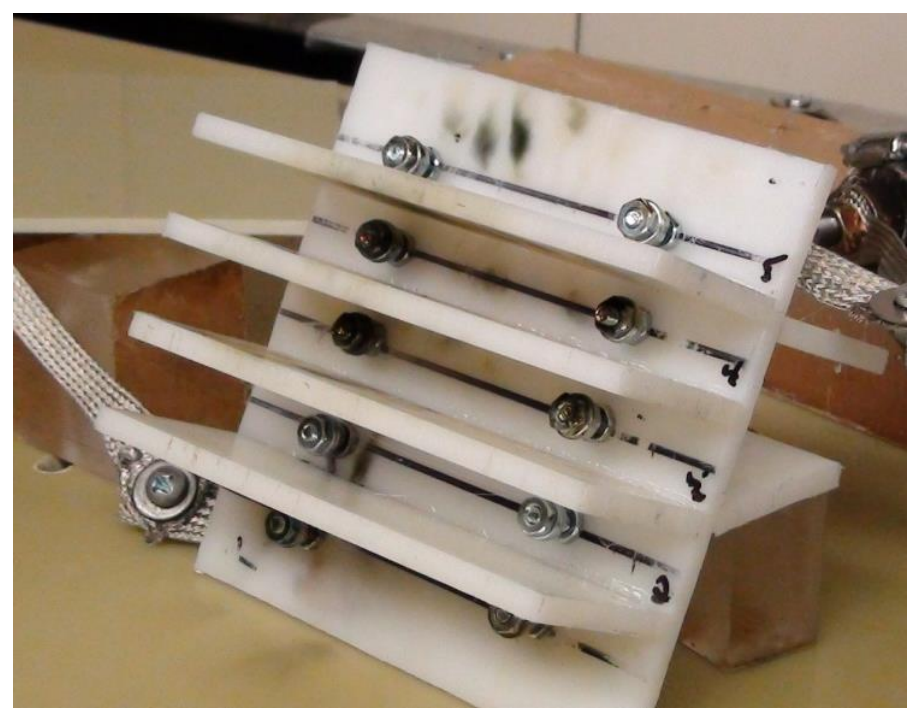

Figure 2.9 Series wire fixture with five channels

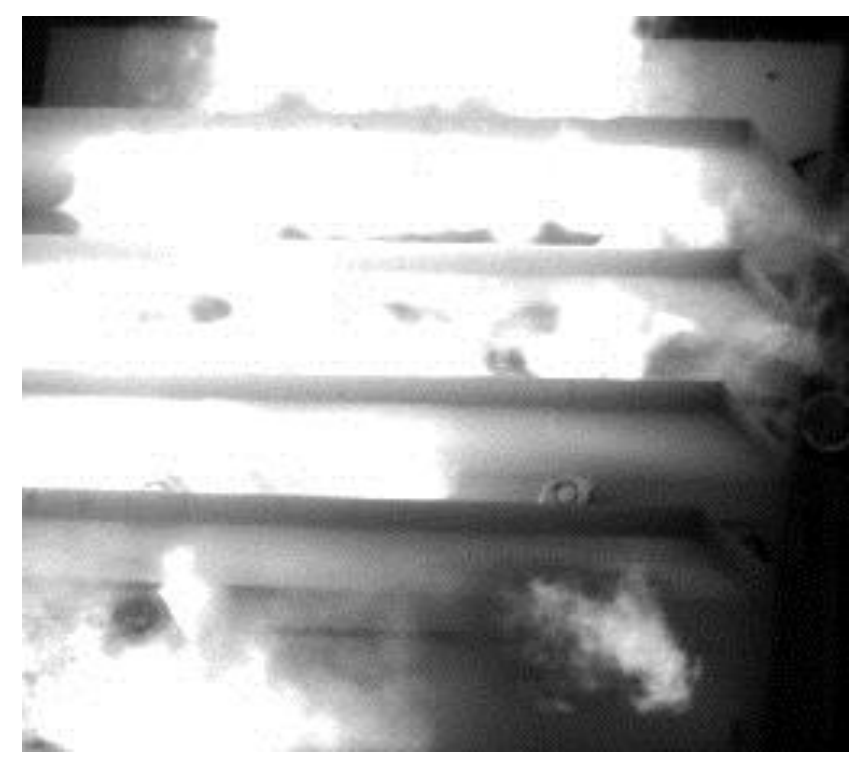

Figure 2.10 Series wire discharge 
This discharge showed that a long series wire could be driven to explode by the pulsed power circuit and that the duration of this discharge was adequate for interaction with a shock wave. The geometry of this discharge would require redesign for interaction experimentation.

Another configuration tested was a 'honeycomb' fixture in which several parallel exploding wires were confined in isolated tubes, restricting expansion of the plasma discharge in the radial direction. The fixture, shown in Figure 2.11, consists of four holes drilled in a $0.10 \mathrm{~m}$ long piece of plastic. Four thin wires (one wire per tube) connect the high voltage anode on one side of the fixture with the cathode on the other side of the fixture. In this configuration, the radial expansion of each exploding wire is restricted, forcing more expansion axially through the anode and cathode screens.
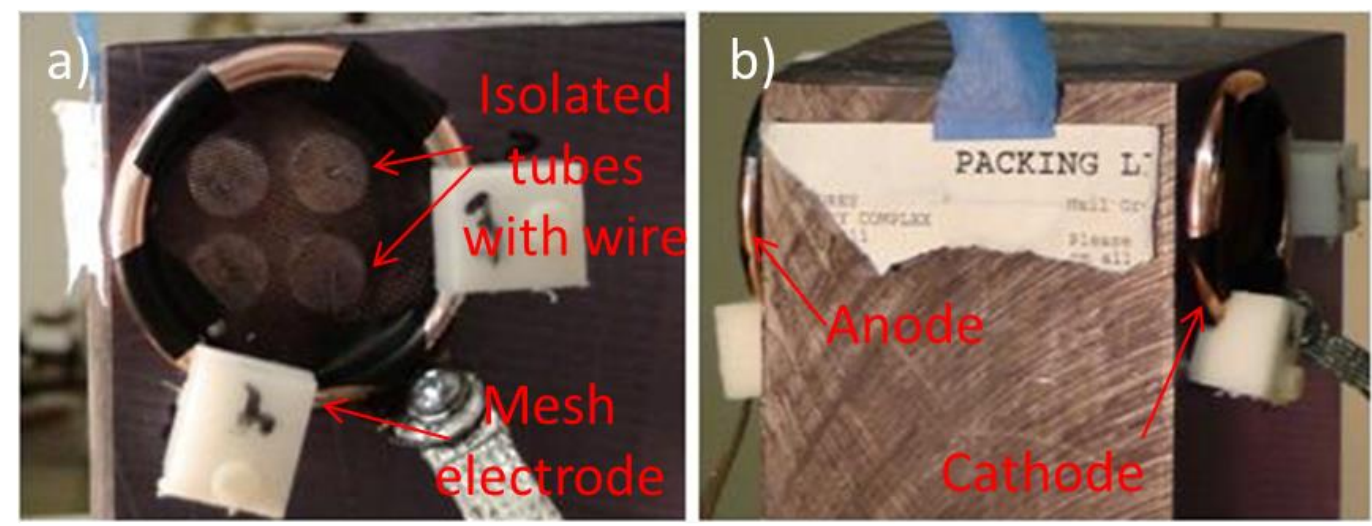

Figure 2.11 'Honeycomb' fixture, a) on axis of wire, b) side-axis perspective

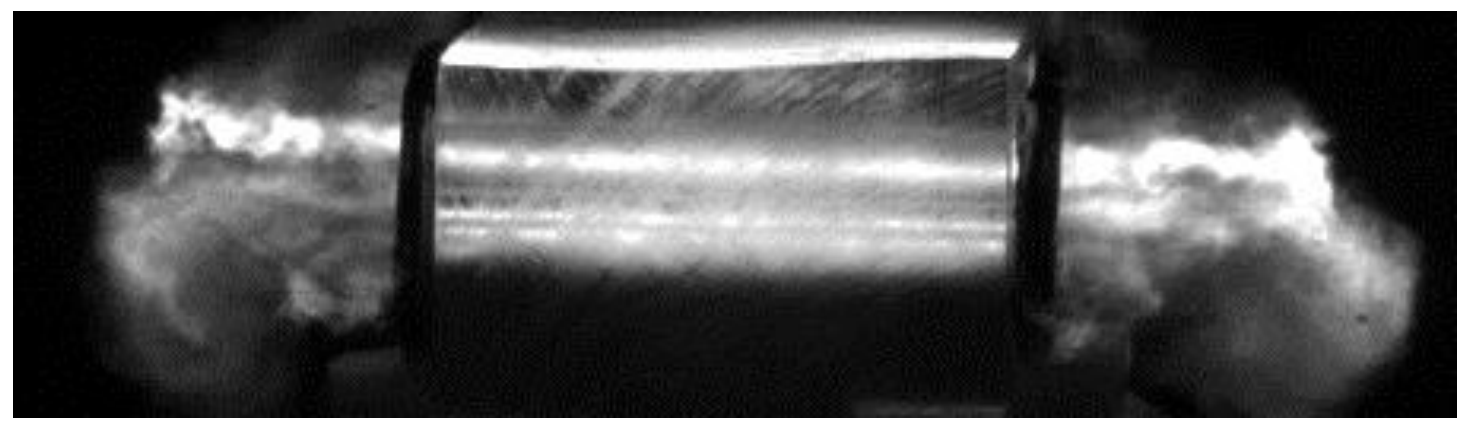

Figure 2.12 Image of the exploding wire discharge from the 'honeycomb' fixture 
Figure 2.12 shows the resultant discharge, which is roughly $400 \mu$ s in duration, and has a considerable volume (the plastic fixture is $0.10 \mathrm{~m}$ in length, thus the discharge is at least $0.20 \mathrm{~m}$ in length). This plasma source was identified as a viable option for interaction with shock waves.

\subsubsection{Early Experiments with the Toroidal Air Plasma}

Several modifications were made to the honeycomb fixture which became the first experiments with a toroidal air plasma, or TAP. The test fixture was modified to have just one tube and one wire linking the high voltage anode and cathode. A secondary acceleration region was added to the fixture as an attempt to impart more energy into the plasma after the main circuit had discharged through the exploding wire. A second stainless steel screen was placed beyond the cathode of the test fixture with an air gap between the cathode and the accelerator screen. As the plasma from the exploding wire expanded out of the fixture, it filled the gap between the two screens, closing the acceleration circuit, and discharging a set of electrolytic capacitors through the plasma. The test fixture and a time-lapsed photograph of the discharge are shown in Figure 2.13.
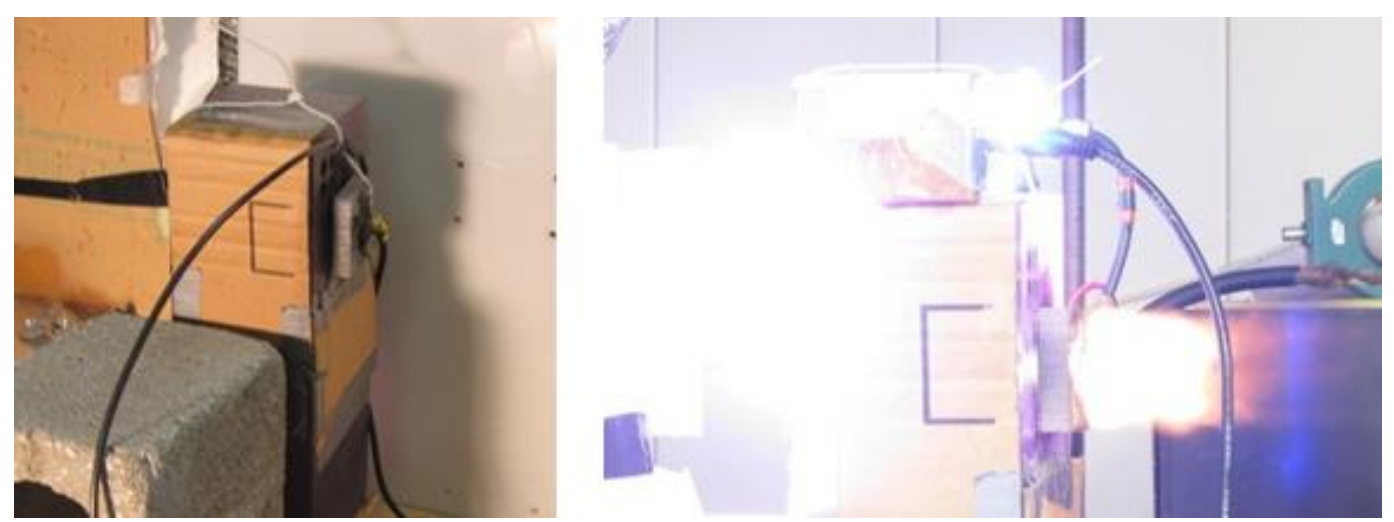

Figure 2.13 Early fixture for the TAP (left) and time-lapsed photograph of the discharge 
The accelerator region was shown to enhance the size and duration of the plasma discharge significantly. The charge voltage on the accelerator capacitor bank was varied to explore the size of the discharge. Early results were inconsistent, with some tests forming large discharges enduring for several milliseconds, while some tests were more comparable to the plasmas seen out of the un-accelerated honeycomb fixture. These inconsistencies were found to be due to faulty electrical connections.

Several experiments were conducted to ascertain reasons for the inconsistent results. Increasing to two lengths of wire in the tube, one continuous wire from anode to cathode and back to the anode, greatly increased the reproducibility of the discharge. With a more reproducible discharge, a series of experiments was conducted to optimize the size and duration of the plasma. It was determined that the most consistent results were produced when the accelerator circuit was charged to $250 \mathrm{~V}$. In an attempt to further enhance the discharge, the high voltage anode was changed from a copper screen to a solid brass plate. This restricted the discharge from expanding out of the anode side of the fixture. This further increased the size of the discharge, and the first images of a toroid shape were recorded, as shown in Figure 2.14. These images were recorded with a high speed camera facing on-axis with the exploding wire during the discharge. Each frame was recorded with a $1 \mathrm{~ms}$ period. Additional side images of the discharge are shown in Figure 2.15. 


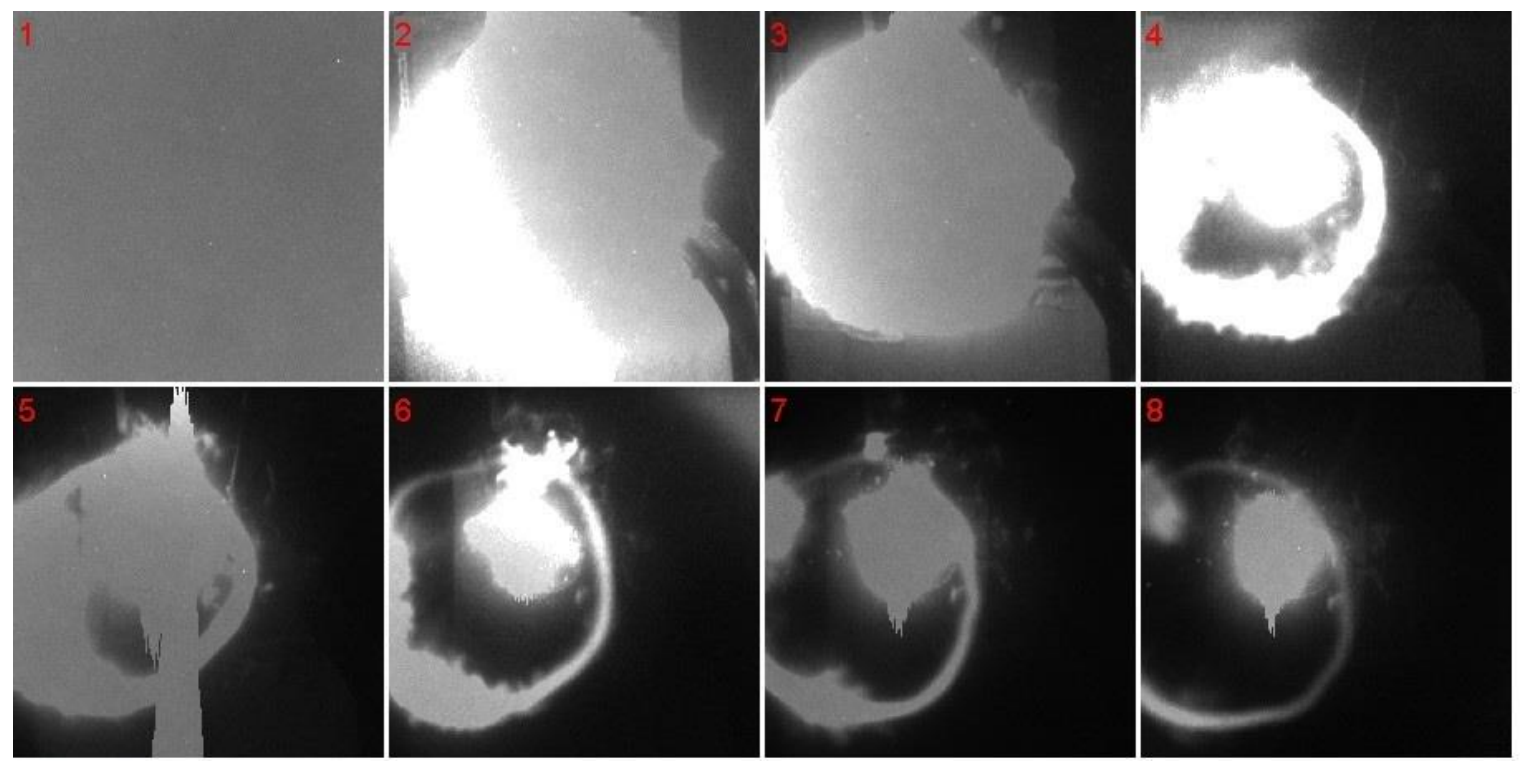

Figure 2.14 Images of the toroidal air plasma from initial experiments (1 ms period)
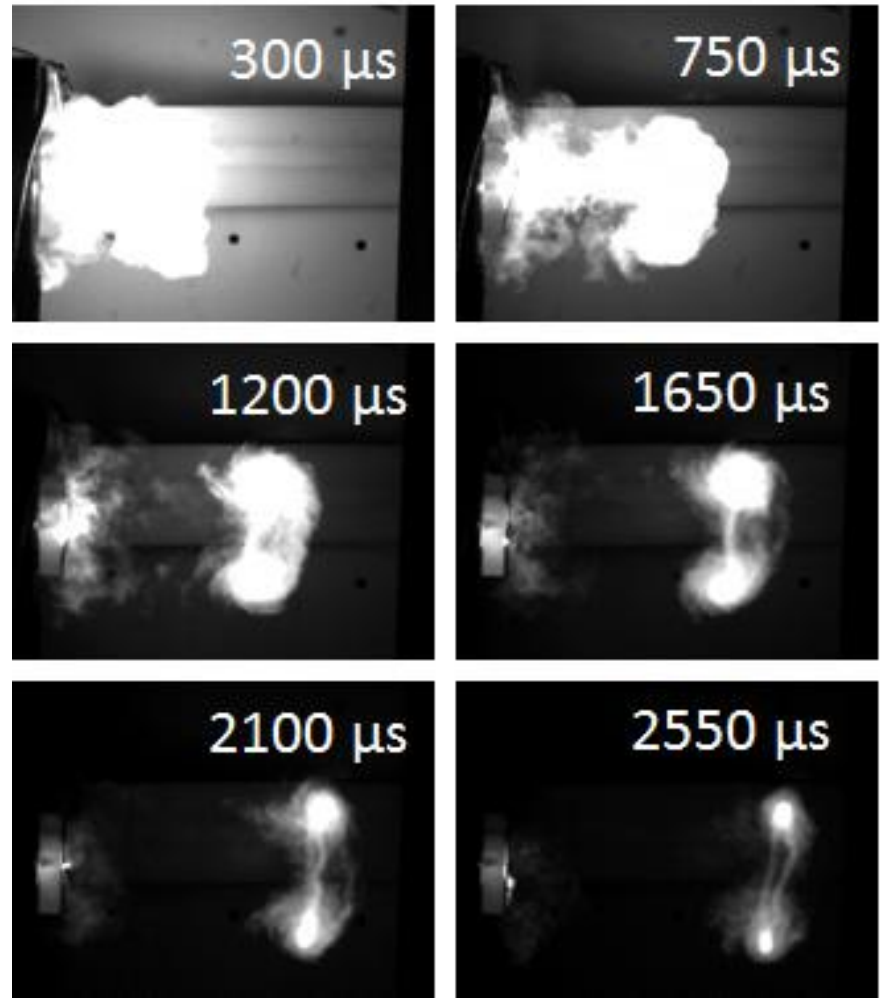

Figure 2.15 Images of the toroidal air plasma labeled with time from vaporization 


\subsubsection{Additional Plasma Source Experiments and Discussion on the Selection of the}

\section{TAP for Interaction Experiments}

After observing the TAP discharge, many experiments were designed to optimize the plasma source for interaction experiments. This process involved experimentation with the geometry of the test fixture, and designing new plasma sources that incorporated some of the features of the TAP fixture. Several of these experiments are presented in Chapter 6. One such new plasma source investigated after the observation of the TAP will be discussed here.

A variation on the series wire discharge was fabricated which incorporated a long wire in a series of channels with an accelerator region, as shown in Figure 2.16. As the exploding wire discharge expands out of the channels, it passes through an acceleration region similar in configuration to that which was used to produce the TAP. This configuration was designed in order to produce a large planar surface area of plasma with the long duration of the TAP. The fixture, shown in Figure 2.16 with and without accelerator screens, is about $0.115 \mathrm{~m} \times 0.127 \mathrm{~m}$ and contains 5 channels, for a total wire length of about $0.50 \mathrm{~m}$.
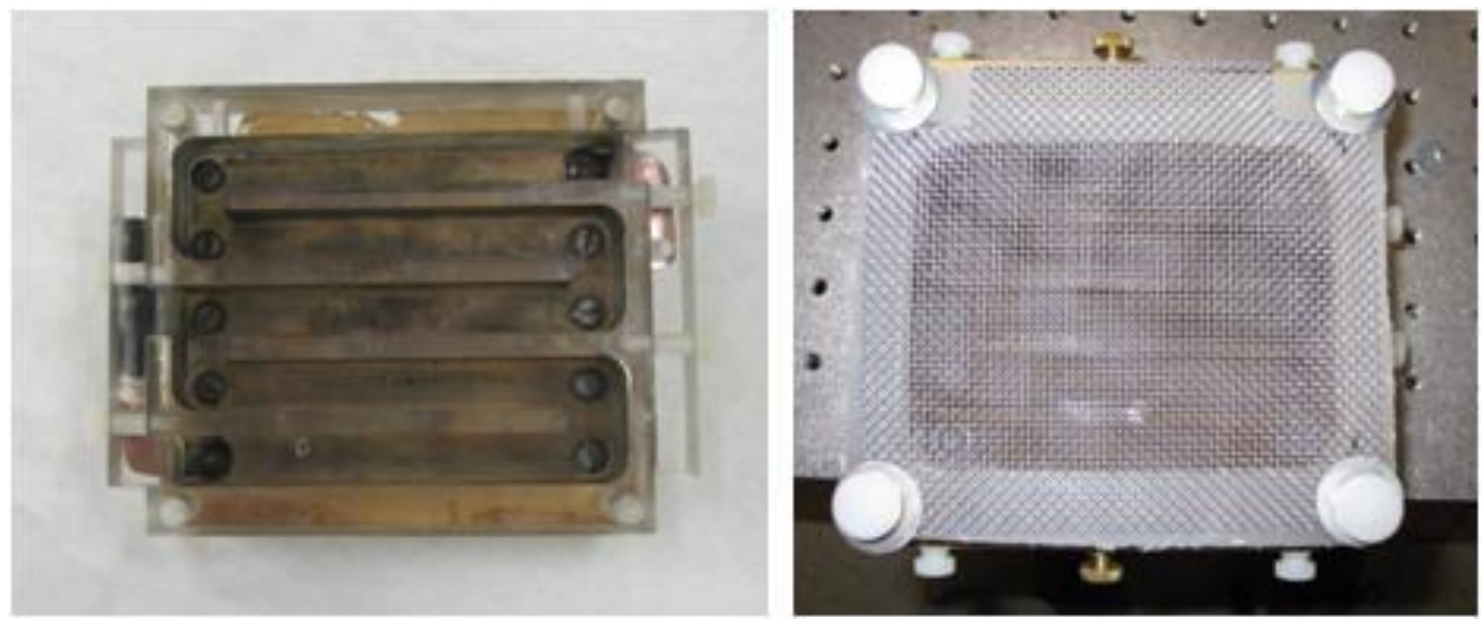

Figure 2.16 The 'serpentine fixture' with (right) and without (left) the accelerator screen 
The exploding wire discharge from this 'serpentine' fixture is shown in Figure 2.17. The first image on the top left is a setup picture recorded by the camera before the experiment. At $339 \mu \mathrm{s}$, the plasma discharge has partially expanded out of the fixture and through the accelerator region. A vortex motion was observed as the plasma expands out of the fixture. It is also apparent that the channels contain plasma even as late as $1,539 \mu \mathrm{s}$, as shown in the final image of Figure 2.17. The bulk of the plasma detaches from the stationary plasma in the accelerator region and the fixture channels, much like the plasma in the TAP. However, unlike the TAP, the plasma dissipates quickly, never enduring for longer than $2.25 \mathrm{~ms}$ in the five experimental trials conducted.
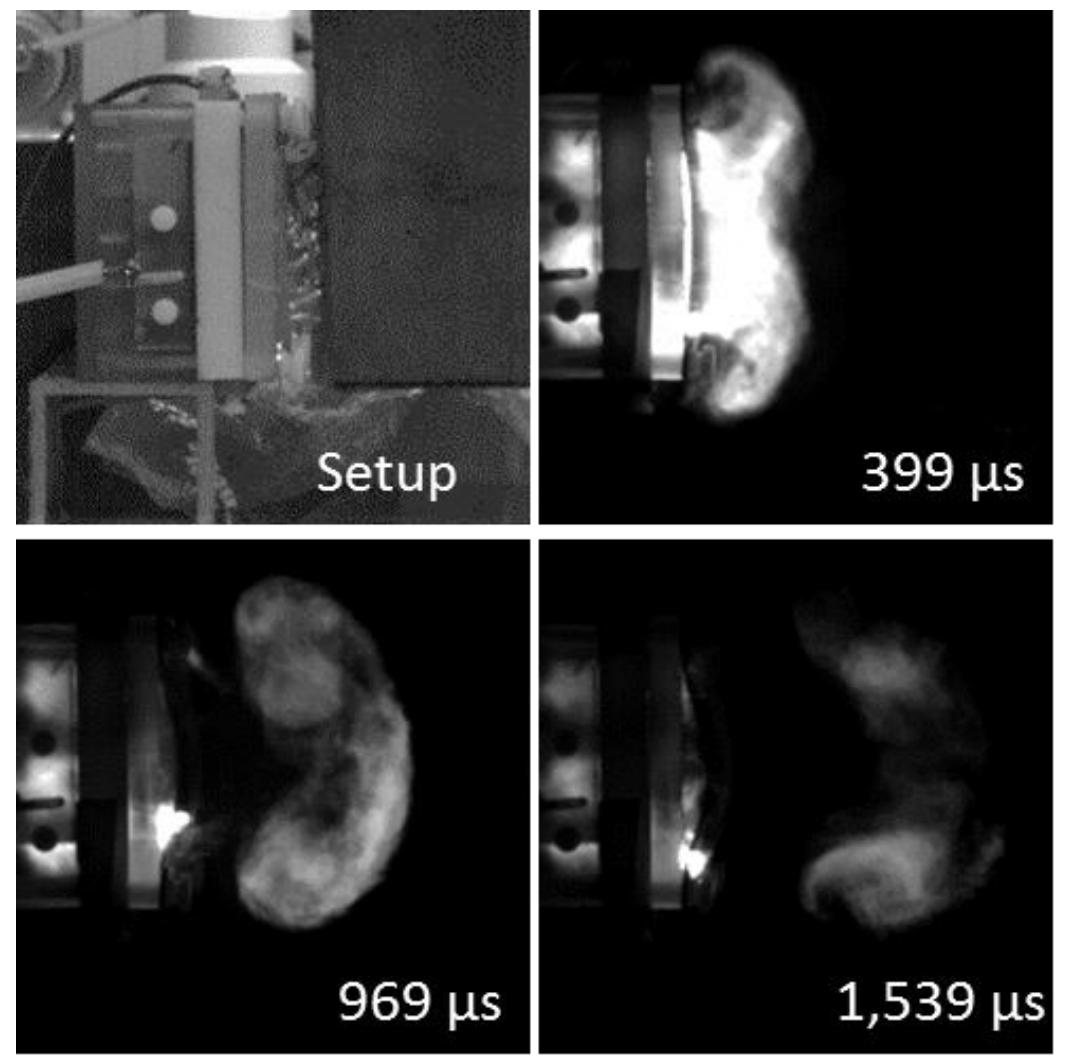

Figure 2.17 High speed images of the discharge from the 'serpentine' fixture

These experiments represent an attempt to find the optimum plasma source for interaction with shock waves. At the present state of this research, the TAP discharge remains the best option because of its longevity and large volume. The TAP discharge 
was chosen as the most viable candidate for mitigating shock waves in air at atmospheric pressure.

\subsection{Previous Research Related to the TAP Discharge}

Self-sustaining plasmas, such as the TAP, are often referred to as plasmoids. A plasmoid is a coherent structure of plasma and electromagnetic fields, typically in a spherical or toroidal shape [43]. Since the late 1950s plasmoids have been generated in various laboratories and have been studied experimentally [44-46]. Many laboratory studies using toroid plasmas were motivated by potential applications in nuclear fusion, the most widely known of which is the Tokamak, where a magnetic field is used to confine a plasma into the shape of a torus [44]. Two examples of early laboratory toroidal plasmas not directly involved in nuclear fusion research are explained further below $[45,46]$.

Lingberg et. al used a plasma gun in low pressure argon (40-400 Pa) with a radial magnetic field to produce magnetized plasma rings in the late 1950s [45]. In these experiments, as the plasma gun fires, the ionized gas is pushed towards the muzzle of the gun. It then encounters a static magnetic field, which is directed radially. As the plasma interacts with the magnetic field, the plasma expands toward the outer walls of the drift tube, shown in Figure 2.18, and produces a plasma ring which travels down the drift tube. These experiments were conducted in hydrogen gas at pressures of 40-400 $\mathrm{Pa}(0.03-3$ Torr). Figure 2.19 is a Kerr-cell photograph of the discharge taken $4 \mu$ s after the ignition of the plasma gun. The front of the plasma travels the length of the drift tube in about $9 \mu \mathrm{s}$, and a toroidal current is measured in the plasma by a magnetic flux loop for approximately $40 \mu \mathrm{s}$ [45]. 


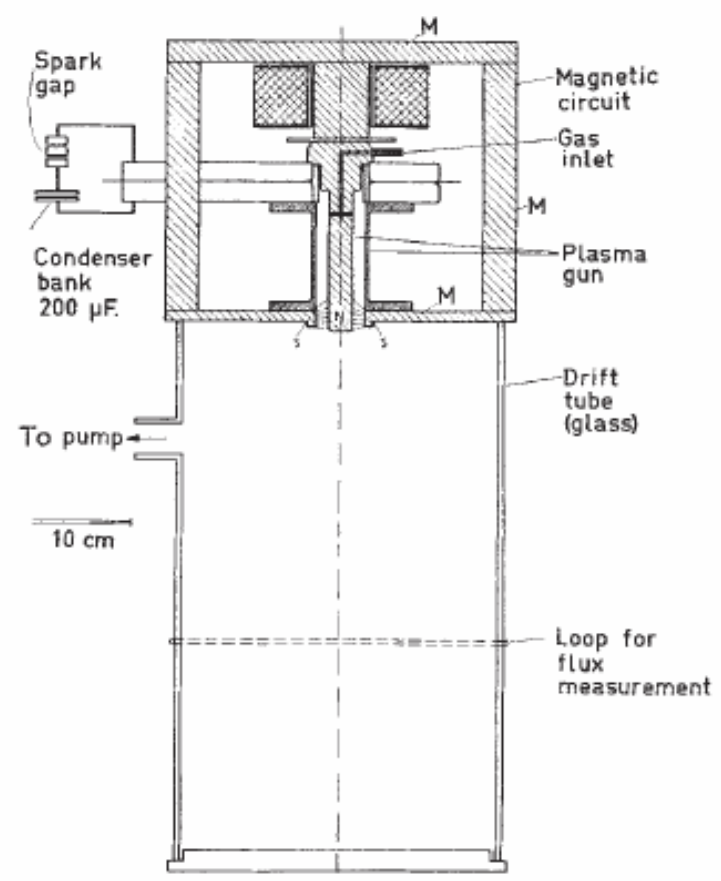

Figure 2.18 Plasma gun with radial magnetic field used to produce plasma rings in hydrogen gas at 40-400 $\mathrm{Pa}$ [45]

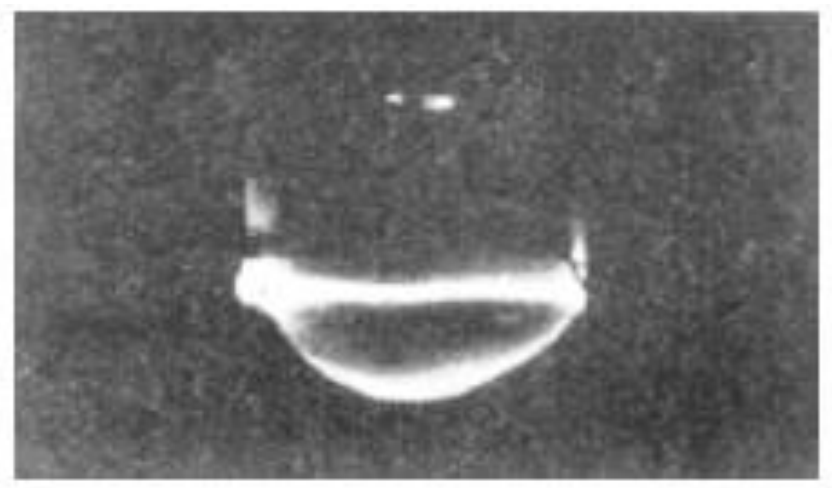

Figure 2.19 Kerr-cell photograph of a magnetized plasma ring [45]

In another study, Wells used a $\theta$-pinch plasma gun with a cold argon gas at $13 \mathrm{~Pa}(0.1 \mathrm{Torr})$ to observe a series of plasma vortex rings [46]. The plasma gun was conically shaped with a DC solenoid acting as a guide field. A preionized cold argon gas was injected into the coil, and when the field was energized, three ring-shaped structures move down the guide. Wells goes on to suggest that these observed rings, shown in Figure 2.20, are plasma vortex rings satisfying what he terms 'magneto-vortex' equations 
derived from steady state, planar flow conditions of the equation of motion for a nonviscous, incompressible fluid (a form of Equation 2. 16) [46].

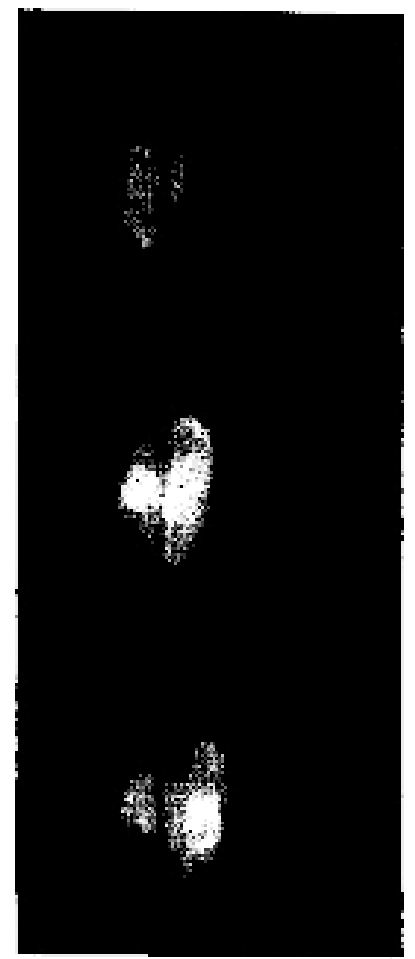

Figure 2.20 Framing camera images of plasma vortex rings in argon gas at 0.1 Torr [46]

Many of these early observations of plasmoids have been used as an explanation of the natural electric phenomenon, ball lightning [47, 48]. Ball lightning is a term that refers to reports of luminous spherical or ring shaped objects typically observed during thunderstorms $[47,48]$. Precise conditions for ball lightning to occur are still unknown, and the phenomenon has rarely been observed, thus properties of ball lightning plasmas have not been measured experimentally. Proposed theoretical models of ball lightning typically involve plasmoid MHD models [47 - 51].

Wooding suggests a model that explains ball lightning as a plasma vortex ring [48]. As suggested, if the plasma possesses vorticity and a vortex ring forms, then the centrifugal force due to the vorticity is balanced by a magnetostatic pressure [48, 52]. 
Wooding goes on to discuss how a lightning event could create conditions which bring about plasma vortex rings, or ball lightning.

In more recent experiments, Seward et. al. have observed spinning plasma toroids (SPT's) which endure for more than 200 milliseconds in pressures of 13.3-1333 Pa (0.1-10 Torr) [53, 54]. An SPT is shown in Figure 2.21. In these experiments, an SPT forms from an electric arc that has particular initiating conditions which cause electrons to orbit around the arc's magnetic field. If the magnetic field is strong enough, electrons can be trapped in this orbit, and if enough orbits form around the arc, an internal magnetic field will be generated. These individual orbits can join into a single toroid, similar to that of a classical toroidal solenoid [53]. The author goes on to calculate the forces of equilibrium in the SPT by analyzing the forces acting on discrete particles. This analysis is then used to hypothesize the possibility of SPTs existing at conditions of atmospheric pressure in air as an explanation for ball lightning [53]. Related work analyzes the equilibrium and stability criteria of these discharges, renamed electron spiral toroids (ESTs), with a cold-fluid equilibrium theory [55].

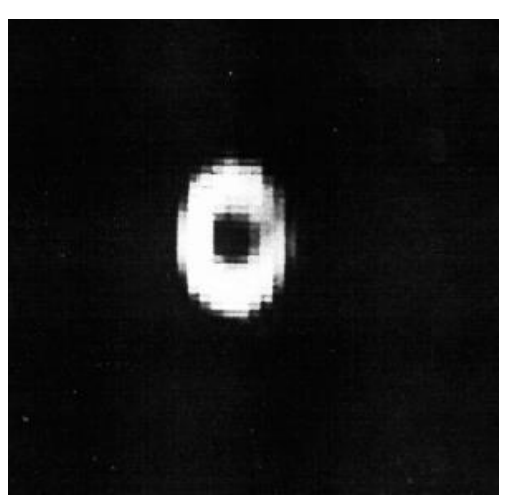

Figure 2.21 A stable plasma toroid observed in an arc discharge experiment [53]

In other experiments, Oreshko and Mavlyudov have observed high-current vortex discharges at atmospheric pressure [56]. The discharge is formed by a breakdown between a pair of specially designed two-electrode cells. A $11.25 \mathrm{~kJ}, 15 \mathrm{kV}$ capacitor 
bank discharges across the electrode cap with a peak current of $100 \mathrm{kA}$. Hot plasmoids $500 \mu$ s in duration generated by the high-current vortex discharges were observed to pass through 0.005-m-thick glass [56].

As an example of experiments which are similar in geometry to the TAP fixture (but without an accelerator grid), Kim et. al. have investigated a capillary discharge in open air to measure energy lost in the discharge [57]. The plasma injector, shown schematically in Figure 2.22, is $0.015 \mathrm{~m}$ long and $0.004 \mathrm{~m}$ in diameter. A nickel wire of $0.2 \mathrm{~mm}$ diameter is used as an exploding wire to initiate the discharge. The resultant ejected jet from the capillary discharge reaches about $0.50 \mathrm{~m}$ at $3.68 \mathrm{~ms}$ and is shown in Figure 2.23, but does not form a toroid. The study was primarily an effort to analyze the power balance between ohmic heating and black body radiation of the discharge [57]. This work is in support of the development of the electrothermal-chemical (ETC) gun.

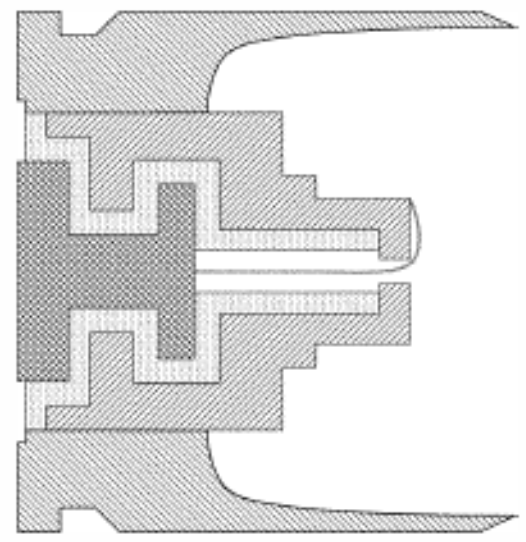

Figure 2.22 Schematic diagram of the plasma injector for capillary discharge [57] 


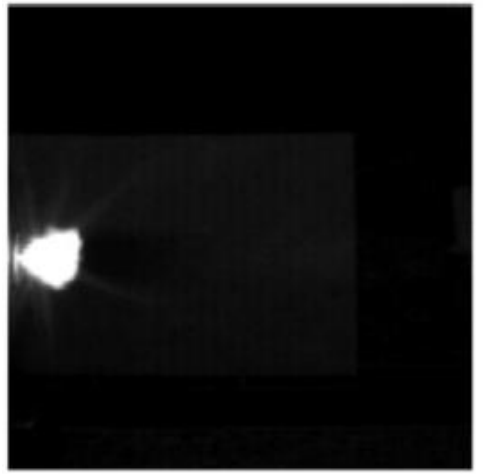

(a)

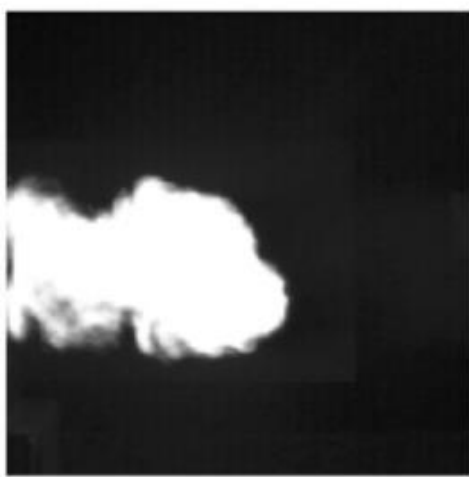

(c)

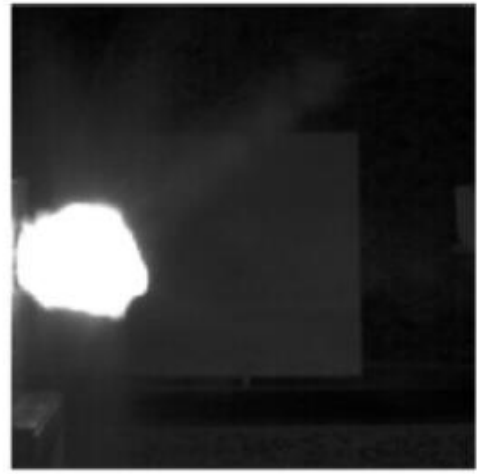

(b)

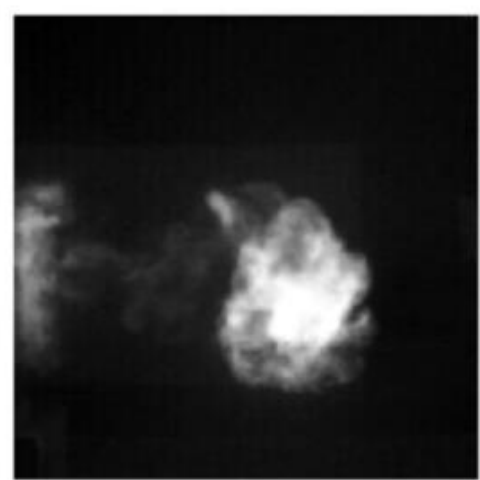

(d)

Figure 2.23 Images of the ejected jet of the capillary discharge at times a) $170 \mu \mathrm{s}$, b) $440 \mu \mathrm{s}$, c) $1.52 \mathrm{~ms}$, and d) $3.68 \mathrm{~ms}$, the dimension of the images is $0.75 \mathrm{~m} \times 0.75 \mathrm{~m}$ [57].

\section{References for Chapter 2:}

1. K. Nishikawa and M. Wakatani, Plasma Physics, Springer-Verlag, Heidelberg, Germany, 2000.

2. M.A. Lieberman and A. J. Lichtenberg, Principles of Plasma Discharges and Materials Processing, Wiley, New York, 2005.

3. F. Chen, Introduction to Plasma Physics and Controlled Fusion, vol. 1, Plenum Press, New York, 1984.

4. P. Lorrain, et al., Magneto-Fluid Dynamics: Fundamentals and Case Studies of Natural Phenomena, Springer, New York, 2006.

5. D. Schnack, "Magnetohydrodynamics," Course Lectures, University of Wisconsin Madison, [online] available: http://www.physics.wisc.edu/grads/courses/726f07/index.html.

6. E.E. Kunhardt, "Generation of Large-Volume, Atmospheric-Pressure Nonequilibrium Plasmas," IEEE Trans. Plasma Sci., vol 28, no 1, 2000.

7. A. Schütze, et al., "The Atmospheric-Pressure Plasma Jet: A Review and Comparison to Other Plasma Sources," IEEE Trans. Plasma Sci., vol 26, no 6., 1998

8. F.Massines, et al., "Experimental and Theoretical Study of a Flow Discharge at Atmospheric Pressure Controlled by Dielectric Barrier," J. Appl. Phys., vol. 83, no. 6, 1998. 
9. L. J. Calvez, "A Novel Power Supply for Generating a One Atmosphere Uniform Glow Discharge Plasma (OAUGDP®)," Ms. Thesis, University of Tennessee, Knoxville, TN, 2007.

10. S.S. Harilal, et al., "Confinement and Dynamics of Laser-Produced Plasma Expanding Across a Traverse Magnetic Field," Phys. Rev. E, vol. 69, 2004.

11. J.F. Kielkopf, "Laser-Produced Plasma Bubble," Phys. Rev. E, vol. 63, 2000.

12. K. H. Schoenbach, et al., "Microhollow Cathode Discharges," J. Vac. Sci. Technol. A, vol. 21. No 4., 2003.

13. K. H. Schoenbach, et al., "High Pressure Hollow Cathode Discharges," Plasma Sources Sci. Technol. vol. 6. pp. 468-477, 1997.

14. D. J. Sturges and H. J. Oskam, "Studies of the Properties of the Hollow Cathode Glow Discharge in Helium and Neon," J of Appl. Phys., vol. 35, no. 10, 1964.

15. G. Schaefer and K. H Schoenbach, "Basic Mechanisms Contributing to the Hollow Cathode Effect," Physics and Applications of Psuedosparks, Plenum Press, New York, 1990.

16. R. Stark and K. H. Schoenbach, "Direct Current High-Pressure Glow Discharges," J. Appl. Phys., vol 85, no 4, 1999.

17. L.D. Biborosch, et al, "Radiation Efficiency of AC-excited Micro Hollow Cathode Discharges", AIP Conf. Proc., vol 812, pp. 329, 2006.

18. L.D. Biborosch, et al., "Excimer Emission of Open and Closed Microhollow Cathode Discharges in Xenon," Rom. Journ. Phys., vol. 50, no. 7-8, pp. 711-722, 2005.

19. D. Hsu and D. Graves, "Microhollow Cathode Discharge Stability with Flow and Reaction," J. Phys. D: Appl. Phys. vol 36, pp. 2898-2907, 2003

20. P. Kurunczi, et al., "Excimer Formation in High-Pressure Microhollow Cathode Discharge Plasmas in Helium Initiated by Low-Energy Electron Collisions," Int. J. Mass Spec., vol. 205, pp. 277-283, 2001.

21. C. Penache, et al., " Experimental Study of High Pressure Glow Discharges Based on MSE Arrays," Proc. Hakone VII, Greifswald, vol. 2, pp .501-5.

22. A. H. Mohamed, R. Block, K. H. Schoenbach, "Direct Current Glow Discharges in Atmospheric Air," IEEE Trans. Plasma Sci., vol 30, no 1, Feb 2002.

23. F. Leipold, et al., "High Electron Density, Atmospheric Pressure Air Glow Discharges," Pwr. Mod. Symp., pp. 130-133, 2002.

24. Robert H. Stark and K. H. Schoenbach, "Direct Current Discharges in Atmospheric Air," Appl. Phys. Lett., vol. 74, no. 25, 1999.

25. F. Leopold, et al., "Electron Density Measurements in an Atmospheric Pressure Air Plasma by Means of Infrared Heterodyne Interferometry ," J. Phys. D: Appl. Phys., vol. 33, pp. 2268-2273, 2000.

26. C. Penache, et al., "Characterization of a High-Pressure Microdischarge using Diode Laser Absorption Spectroscopy," Plasma Sources Sci. Tech., vol. 11, pp. 446-483, 2002

27. W. Xinbing, et al., "High Pressure Micro-Slot Hollow Cathode Discharges," Plasma Sci. \& Tech, vo1.7, no.6, 2005.

28. J. G. Eden, et al., "Microplasma Devices Fabricated in Silicon, Ceramic, and Metal/Polymer Structures: Arrays, Emitters, and Photodetectors ," J. Phys. D: Appl. Phys., vol. 36 , pp. 2869-2877, 2003. 
29. J. Chen, et al., "Microdischarge Device Fabricated in Silicon By Micromachining Technique with Pyramidal Cavity," $11^{\text {th }}$ Int.Conf Solid-State Sensors \& Actuators, Munich, Germany, 2001.

30. S.J. Park, et al., "Silicon Microdischarge Devices Having Inverted Pyramidal Cathodes: Fabrication and Performance of Arrays," Appl. Phys. Lett., vol. 28, no. 4, 2001.

31. S. J. Park, et al.,"Microdischarge Arrays: A New Family of Photonic Devices (Revised)," IEEE J. Select. Topics Quantum Electron., vol. 8, no. 2, 2002.

32. C. Penache, et al., "Micro-Structured Electrode Arrays: A Source of High-Pressure Non-Thermal Plasma," Proc. SPIE Int. Soc. Opt. Eng., ISSU vol. 4460, pp. 17-25, 2002.

33. P. von Allmen, et al, "Ceramic Microdischarge Arrays with Individually Ballasted Pixels" Appl. Phys. Lett., vol. 82. no. 16. 2003.

34. J.W. Frame and J.G. Eden, "Planar Microdischarge Arrays," Electron. Lett., vol. 34, no. $15,1998$.

35. Adam Lodes and Randy Curry, "Atmospheric Glow Discharge Plasmas using a Microhollow Cathode Device," Pulsed Power Conf., pp. 975-979, 2009.

36. K. A. O'Connor, "Compact Power Conditioning and RF Systems for a High Power RF Source," Ms. Thesis, Elec. and Comp. Engr., University of Missouri, Columbia, MO, 2008.

37. W. G. Chace, and H. K. Moore, Exploding Wires, vol I, II, III, IV, New York: Plenum Press Inc, 1959.

38. W. G. Chace and M. A. Levine, "Classification of Wire Explosions," J. Appl. Phys., vol. 31, pp. 1298, 1960.

39. S. I. Tkachenko, et al., "Parameters of Wires During Electric Explosion," Appl. Phys. Lett., vol. 82, pp. 4047-4049, June 9, 2003.

40. V. S. Sedoi, et al., "The Current Density and the Specific Energy Input in Fast Electrical Explosion," IEEE Trans. Plasma Sci., vol. 27, pp. 845-850, 1999.

41. G. W. Anderson and F. W. Neilson, "Use of the "Action Integral" in Exploding Wire Studies," in Exploding Wires. vol. I, W. G. Chace and H. K. Moore, Eds. New York, NY: Plenum Press, 1959.

42. T. J. Tucker and R. P. Toth, "A Computer Code for the Prediction of the Behavior of Electrical Circuits Containing Exploding Wire Elements," Sandia National Laboratories, Albuquerque, NM, SAND-75-0041, 1975.

43. E. Lewis, "Plasmoid Phenomenon," 1996, [online] available: www.padrak.com/ine/ELEWIS2.html, accessed Jan 2012.

44. L. A. Artsimovich, "Tokamak Devices," Nuclear Fusion, vol. 12, pp. 215, 1972.

45. L. Lindberg, et al., "Experiments with Plasma Rings," Nature, vol. 185, 1960.

46. D. R. Wells, "Observation of Plasma Vortex Rings," Phys. Fluids, vol. 5, pp. 1016, 1962.

47. S. Singer, "The Unsolved Problem of Ball Lightning," Nature, vol. 198, 1963.

48. E.R. Wooding, "Ball Lightning," Nature, vol. 199, 1963.

49. V.G. Endean, "Ball Lightning as Electromagnetic Energy," Nature, vol. 263, 1976.

50. A. F. Ranada and J. L. Trueba, "Ball Lightning: An Electromagnetic Knot," Nature, vol. 383, 1996. 
51. J. Brandburg, et al., "The Theory and Simulation of Plasmoid Formation and Propagation," Mission Research Corporation Report, 1989.

52. H. Alfvén and C.G. Fälthammar, "Cosmical Electrodynamics," Oxford University Press, U.K. 1963.

53. C. Seward, C. Chen, K. Ware, "Ball Lightning Explained as a Stable Plasma Toroid," Pulsed Power Plasma Sci., vol.1, pp. 269- 272, 2001.

54. D. C. Seward, et al., "Energy Storage Device," US Patent 6,140,752, Oct. 31, 2000.

55. C. Chen, et al., "Equilibrium and Stability Properties of Self-Organized Electron Spiral Toroids," Phys Plasmas. vol. 8, no. 10., 2001.

56. A. Oreshko and T. Mavlyudov, "The Effect of Hot Plasmoid Generation in HighCurrent Vortex Discharge Under Atmospheric Conditions," IEEE Trans. Plasma Sci., vol. 39, no. 11, 2011.

57. S.H. Kim, et al., "Capillary Discharge in the Open Air," IEEE Trans. Magnetics, vol. 39, no. 1, 2003. 


\section{CHAPTER 3: EXPERIMENTAL TEST BENCH INCLUDING THE PLASMA CIRCUIT AND THE SHOCK TUBE}

The experimental test bench developed for this research program consists of two pulsed power circuits, an accelerator circuit for imparting more energy into the plasma once it has discharged, an exploding-wire triggered shock tube, a central control unit, and a diagnostic suite including a high speed camera. The system is shown as a block diagram in Figure 3.1. This chapter is a detailed account of the experimental test bench. The diagnostic suite will be detailed in Chapter 4.

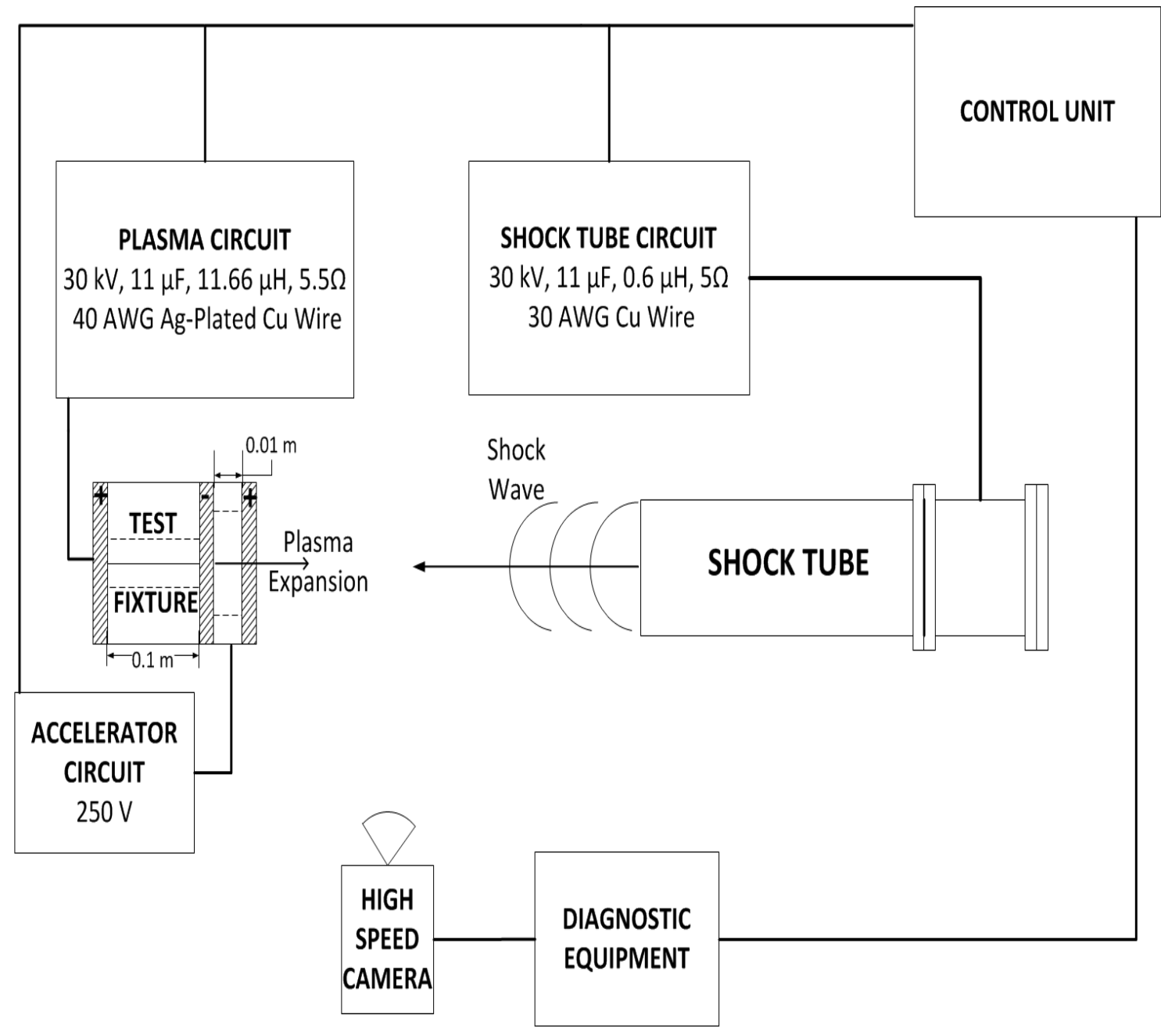

Figure 3.1 Experimental test bench for this research program 


\section{1 Exploding Wire Simulations}

For this research program, we utilized a MATLAB ${ }^{\circledR}$ code developed to simulate the action integral of a wire fuse to model the current, voltage, and resistivity of an exploding wire over time [1]. The model calculates the dynamic resistance of the wire based upon the relationship between resistivity and specific action of the wire, where the current action is predicted by the current and voltage on the exploding wire with respect to time. The model was modified from its original form to simulate an RLC circuit with a dynamic resistance representing the plasma-generating exploding wire [1]. The model has been used to design the pulsed power circuits for this research program. Details of the model and its implementation are given in Appendix A.

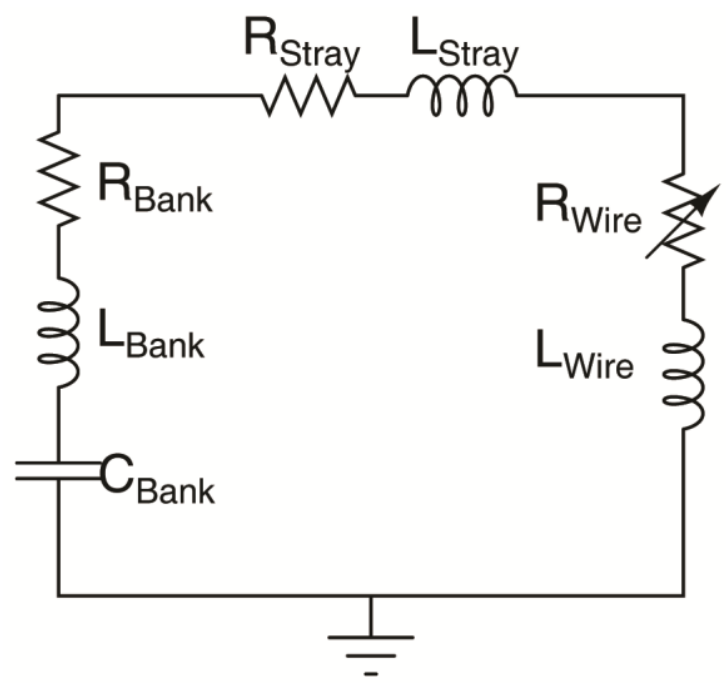

Figure 3.2 Exploding wire driver circuit model

The typical exploding wire driver circuit is shown in Figure 3.2. The primary energy storage capacitor bank is represented by $C_{B a n k}, L_{B a n k}$, and $R_{B a n k}$. In addition to the required capacitance of the capacitor bank, the equivalent series resistance and inductance of the capacitor bank can be included in the program if needed. The exploding wire is represented as a variable resistor, $R_{\text {Wire }}$, and an equivalent series inductance, $L_{\text {Wire }}$, that 
can be included in the simulation or set to zero. Components $R_{\text {Stray }}$ and $L_{\text {Stray }}$ represent any stray circuit resistance and inductance, respectively, as well as any discrete resistors or inductors placed in the circuit external to the capacitor bank and exploding wire. The circuit parameters of a typical experiment to produce the TAP are summarized in Table 3.1 and the wire parameters are summarized in Table 3.2.

Table 3.1 Plasma circuit parameters

\begin{tabular}{|c|c|c|c|c|}
\hline $\mathrm{C}_{\text {bank }}$ & $\mathrm{L}_{\text {bank }}$ & $\mathrm{R}_{\text {bank }}$ & $\mathrm{R}_{\text {stray }}$ & $\mathrm{L}_{\text {stray }}$ \\
\hline $11 \mu \mathrm{F}$ & 0 & 0 & $5.2 \Omega$ & $12 \mu \mathrm{H}$ \\
\hline
\end{tabular}

Table 3.2 Exploding wire parameters of the plasma circuit

\begin{tabular}{|c|c|c|c|c|c|}
\hline $\mathrm{L}_{\text {wire }}$ & $\begin{array}{c}\text { Initial wire } \\
\text { resistivity }\end{array}$ & $\begin{array}{c}\text { Single wire } \\
\text { area }\end{array}$ & $\begin{array}{c}\text { Wire mass } \\
\text { density }\end{array}$ & Wire length & $\begin{array}{c}\text { Number of } \\
\text { wires }\end{array}$ \\
\hline 0 & $1.725 \mathrm{E}-8 \Omega \mathrm{m}$ & $4.87 \mathrm{E}-9 \mathrm{~m}^{2}$ & $8690 \mathrm{~kg} / \mathrm{m}^{3}$ & $0.1016 \mathrm{~m}$ & 2 \\
\hline
\end{tabular}

The following plots are the results of the model for the plasma circuit. Figure 3.3 is the current through the wire which reaches a peak of $5.149 \mathrm{kA}$ at $9.7080 \mu \mathrm{s}$. Figure 3.4 is the voltage across the wire which reaches a peak of $50.65 \mathrm{kV}$ directly before restrike at $2.37 \mu$ s. Figure 3.4 is the wire resistance, and Figure 3.5 is the energy deposited into the wire, with a peak of approximately $50 \mathrm{~J}$. This simulation predicts a sudden drop in resistance associated with re-strike at $2.37 \mu \mathrm{s}$. 


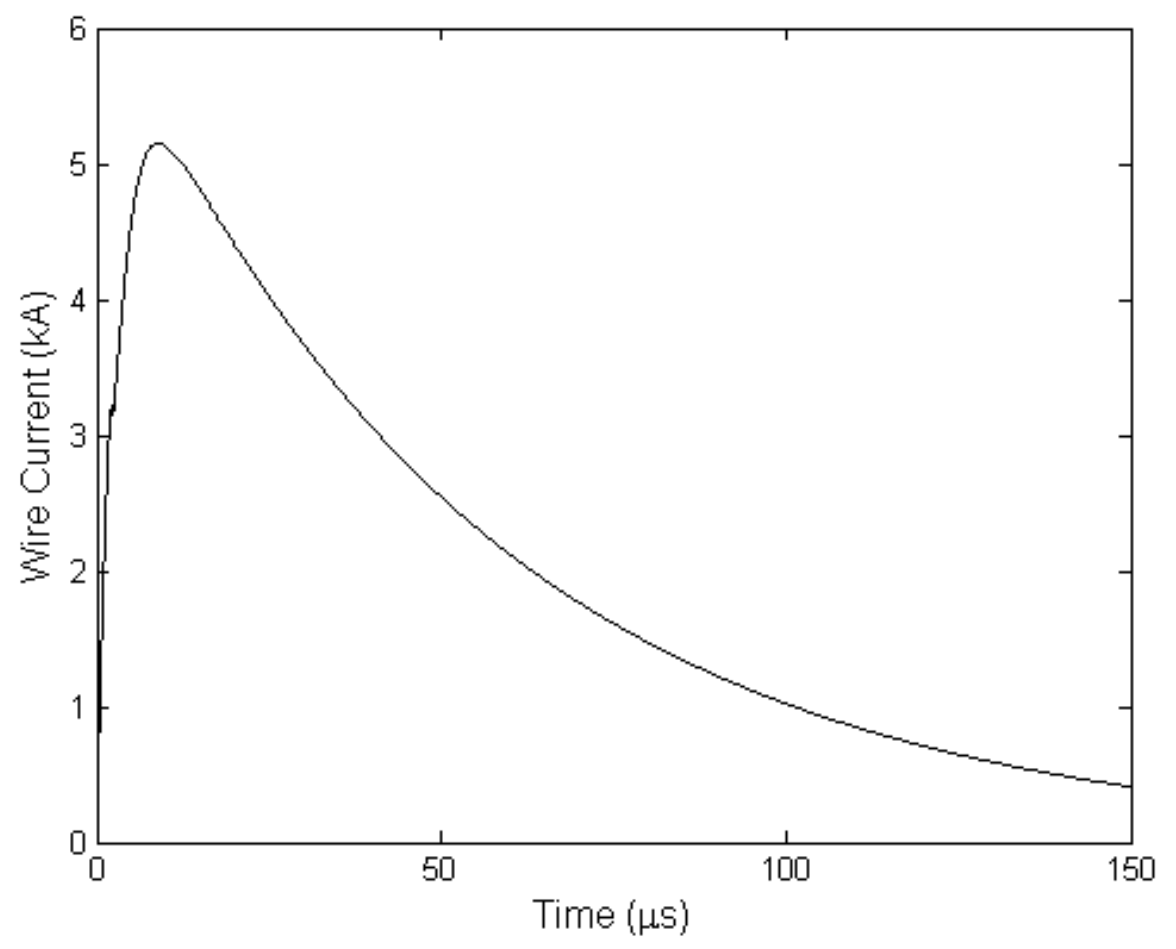

Figure 3.3 Simulated current through exploding wire

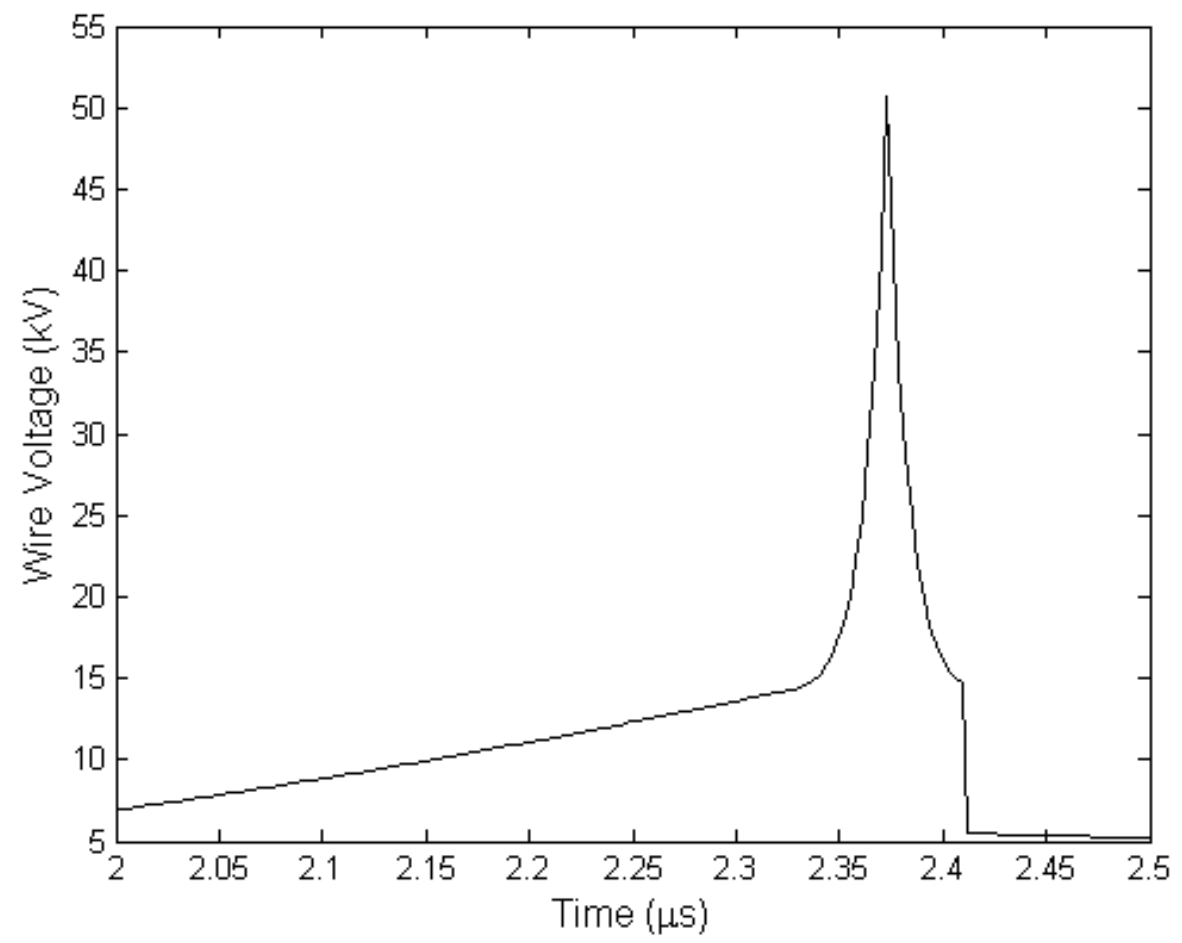

Figure 3.4 Simulated voltage across exploding wire 


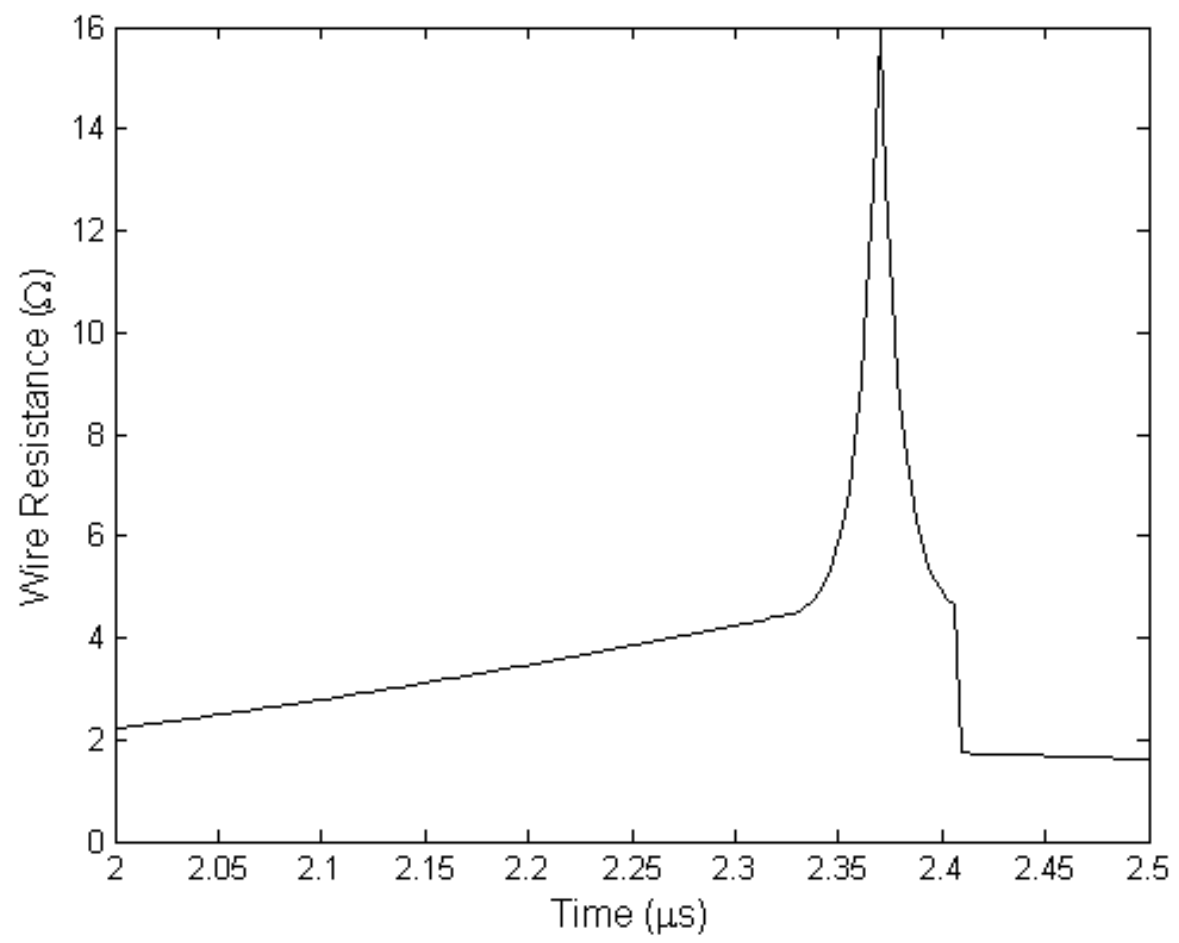

Figure 3.5 Simulated wire resistance

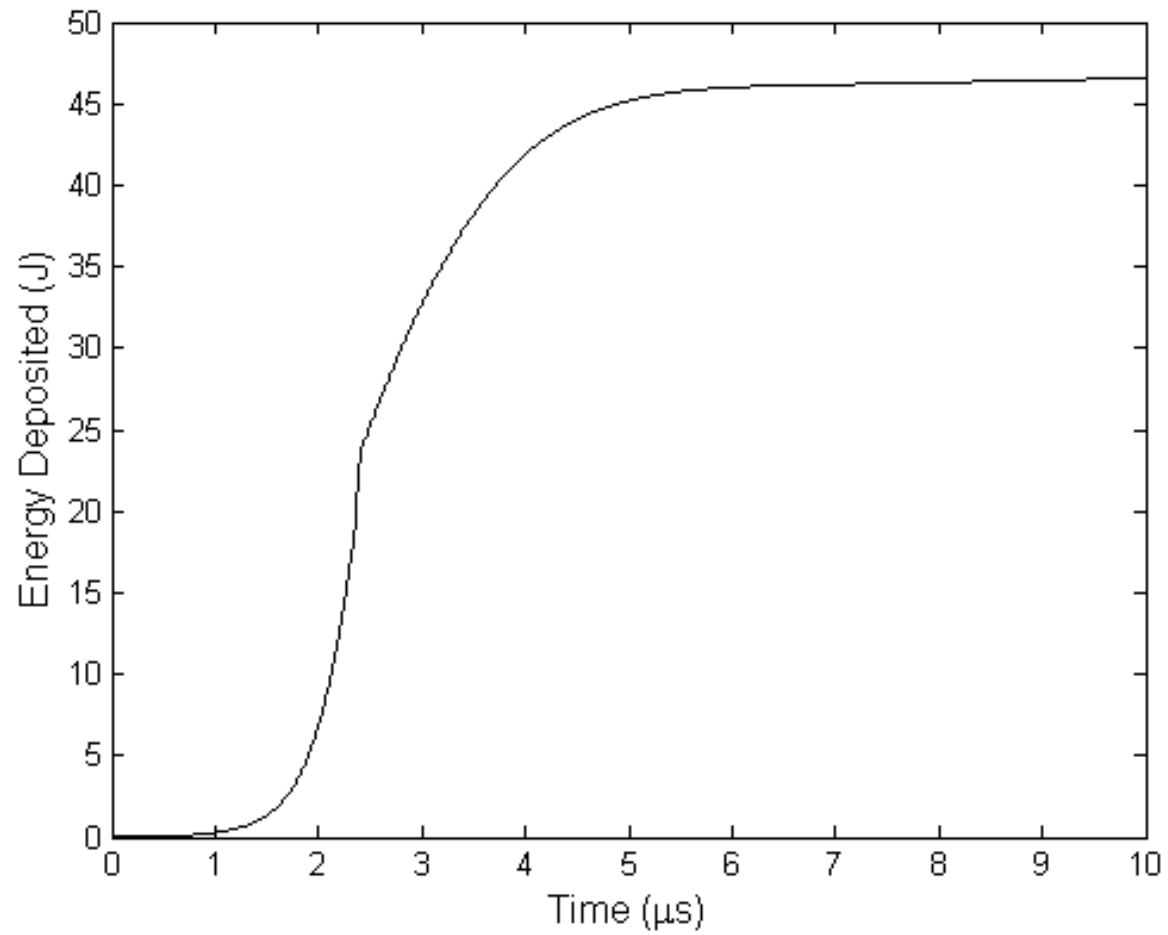

Figure 3.6 Simulated energy deposited into the wire 
This code has been further modified to estimate the electron density of the plasma. To determine this density, the energy deposited in the wire is calculated to determine the energy required to ionize the exploding wire. The number of ionized atoms and the concentration of released electrons are then calculated, leading to an estimate of the electron density within the containing tube for the exploding wire. The simulation calculated an electron density of $3.4 \times 10^{24} \mathrm{~m}^{-3}\left(3.4 \times 10^{18} \mathrm{~cm}^{-3}\right)$. This assumes complete ionization of the wire, which is a reasonable assumption given the large energy deposited into the wire. It also assumes these electrons fill only the confining tube of the plasma geometry. The calculated electron density on the order of $10^{24} \mathrm{~m}^{-3}\left(10^{18} \mathrm{~cm}^{-3}\right)$ is not applicable to later periods of the TAP discharge when it has expanded out of the test fixture. Other analysis of the electron density of the TAP in later stages of evolution will be presented in Chapter 5.

\section{2 Plasma Circuit}

The pulsed power circuit employed at UMC for plasma generation is an RLC circuit with a three-plate pressurized air gap switch, the dynamic wire load, and additional protection and safety circuitry. The basic configuration is shown in Figure 3.7 and the circuit is shown in Figure 3.8.

The circuit employs an $11 \mu \mathrm{F}$ bank of five $2.2 \mu \mathrm{F}$ high voltage capacitors manufactured by Aerovox, Inc. For these experiments, this bank was charged to $30 \mathrm{kV}$ for a total energy storage of $4.4 \mathrm{~kJ}$. The image on the right in Figure 3.8 shows the capacitor bank, which is housed in a grounded steel cart below the other circuit components. A high current copper pipe bus connects the positive terminal of each capacitor to the circuit to minimize resistance and inductance. 


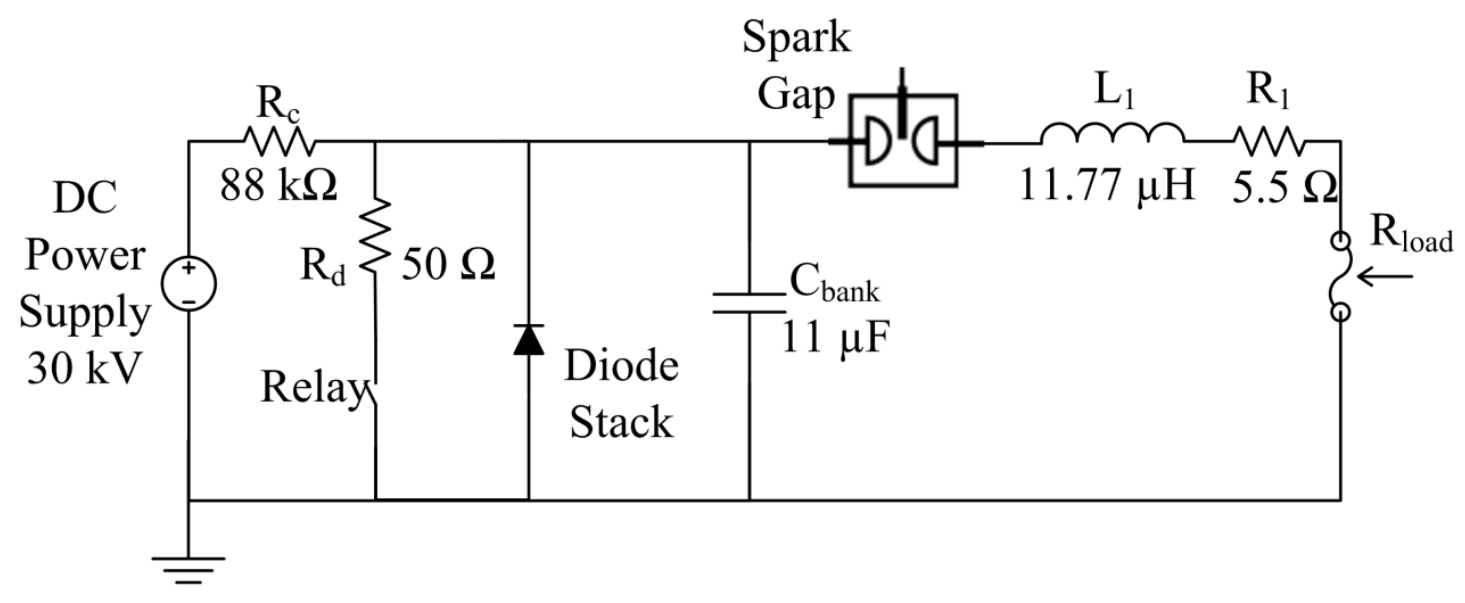

Figure 3.7 Diagram of plasma circuit
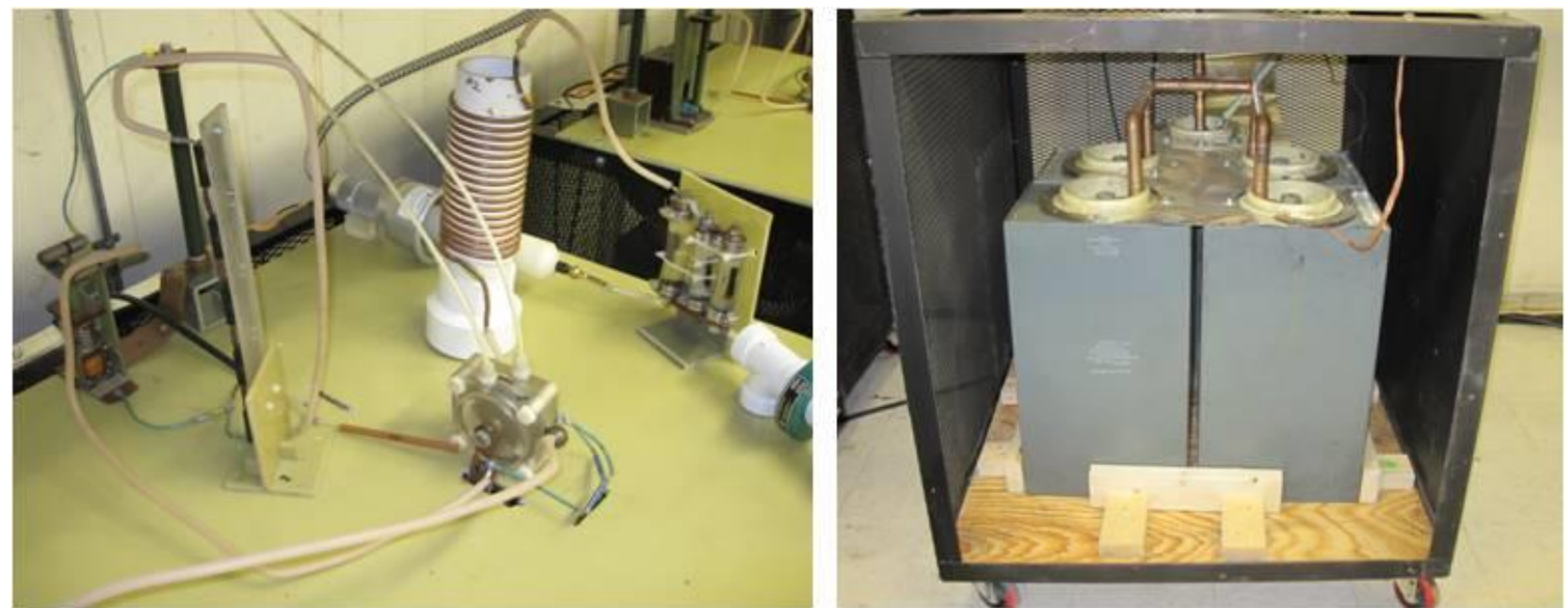

Figure 3.8 Plasma circuit (left) with high voltage capacitor bank (right)

The high voltage capacitor bank is charged with a $50 \mathrm{kV}, 6 \mathrm{~mA}$ Glassman constantvoltage, constant-current power supply through an $88 \mathrm{k} \Omega$ ceramic charging resistor. The circuit is also equipped with two safety features: a shorting relay and a protection diode stack. The shorting relay is designed to handle a fully charged capacitor bank discharge through a $50 \Omega$ ceramic resistor to ground. The circuit is shorted through the relay and through a shorting rod directly across the capacitors when not in use. To protect the power supply and the capacitors from transient current reversals, a stack of high voltage diodes was added to the circuit across the capacitor bank. 
A L-3 communications, T-670 series three-plate spark gap switch was used in this circuit. Spark gaps are commonly used in pulsed power applications due to their quick closure times, low breakdown jitter, and high breakdown current capability [2]. A basic spark gap consists of two electrodes separated by a distance $d$, which can hold off an electric potential $V$. The maximum electric field held off for a particular gap spacing is described by Paschen's Law [3, 4]. Equation 3.1 is Paschen's Law for air, where $X[\mathrm{~m}]$ is the spacing between the electrodes, $p$ [Torr] is the gas pressure, and $T[\mathrm{~K}]$ is the temperature [3].

$$
\mathrm{E}_{\max }=\frac{\mathrm{V}_{\max }}{X}=2420\left(\frac{293}{T}\right)\left(\frac{p}{760}\right)+608 \sqrt{\frac{1}{X}\left(\frac{293}{T}\right)\left(\frac{p}{760}\right)}
$$

Since different gases have different insulating properties, the relative electric strength of the insulating medium must be accounted for when using gases other than air in spark gap switches. A Paschen curve, such as the one shown in Figure 3.9, depicts the relationship between peak breakdown voltage and the product of gas pressure and electrode gap spacing for various gases [5]. For more background on the well-studied field of high voltage spark gap switches, please refer to [2-7].

The T-670 switch was designed for a $7.5^{*} 10^{-3} \mathrm{~m}$ gap spacing [6]. For these experiments, the switch was pressurized with compressed air to hold off the $30 \mathrm{kV}$ charge from the capacitors. When applying the trigger voltage to the mid plane of the spark gap, the electric field between the outer electrodes is enhanced. This results in the gas breakdown between the electrodes, and the capacitors discharge into the load of the circuit, which in this case is an exploding wire. The T-670 specifications call for the operating voltage to be approximately $75 \%$ of the self-breakdown values, calling for a pressure of 10 psi of compressed air to hold off $40 \mathrm{kV}$ and thus allowing for safe 
operation at $30 \mathrm{kV}$. The $\mathrm{T}-670$ also requires a trigger voltage of $50 \%$ or more of the operating voltage for proper field enhancement and reliable switch closure.

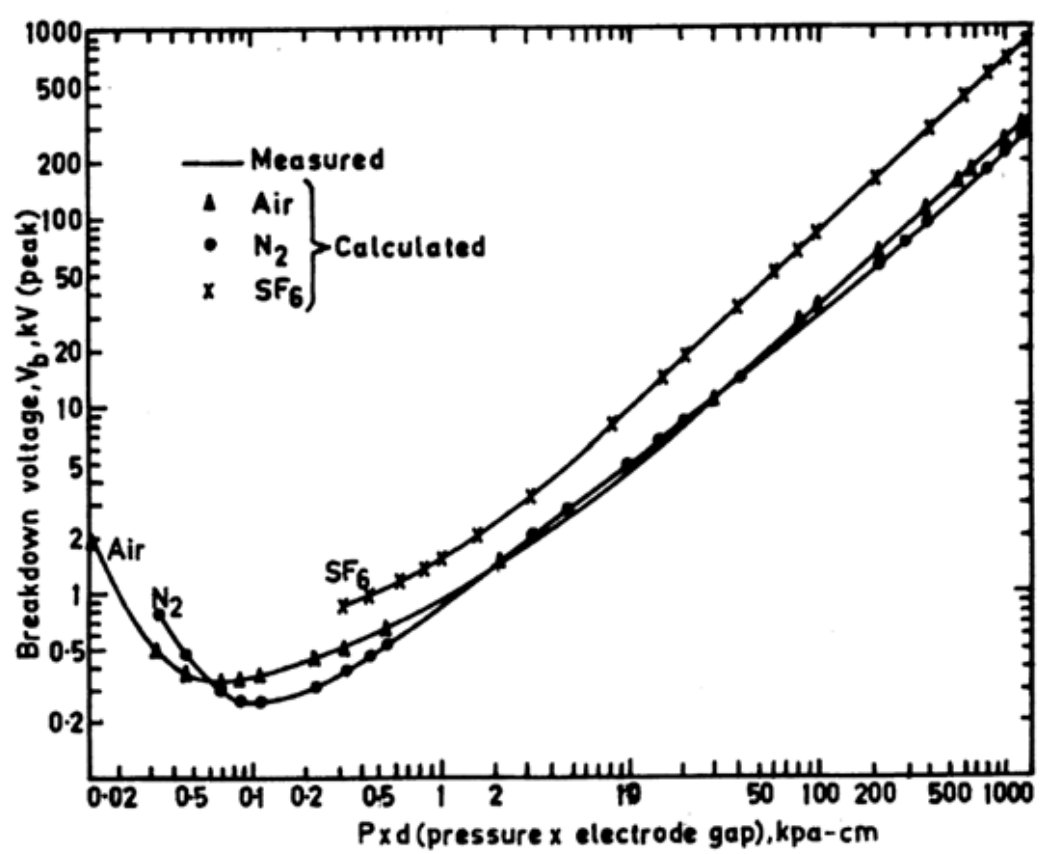

Figure 3.9 Paschen breakdown curve [5]

For these experiments the switch was pressurized to 13 psi with compressed air to ensure voltage breakdown did not occur before the trigger. At an operating voltage of $30 \mathrm{kV}$, a triggering voltage of at least $15 \mathrm{kV}$ is required to adequately trigger breakdown. The triggering circuit which provides this pulse to the trigger electrode will be presented in Section 3.7 and Appendix B.

In order to protect the capacitor bank as well as limit the amplitude and extend the pulse width of the current through the wire, a discrete inductor and resistor were included in the circuit. In practice, the pulse width of the current through the wire is approximately $46 \mu$ s with the $11.77 \mu \mathrm{H}$ inductor and a $5.5 \Omega$ resistor. These elements were sized with the assistance of the circuit modeling presented previously. The air core inductor consists of a $6.35^{*} 10^{-3} \mathrm{~m}(0.25 ")$ copper pipe wrapped in a single layer around a $0.089 \mathrm{~m}(3.5$ ") diameter PVC and can be seen in the center of the left image in Figure 3.8. To prevent 
flashover, a $6.35^{*} 10^{-3} \mathrm{~m}\left(0.25^{\prime \prime}\right)$ rubber insulating hose was wrapped along with the copper pipe, separating each winding. This inductor was measured to be $11.77 \mu \mathrm{H}$. This inductance is designed to slow the pulse through the exploding wire resulting in a longer duration energy deposition into the plasma.

An aqueous-electrolyte resistor was used in this circuit due to its high power rating, cost efficiency, and tunable resistance. Aqueous resistors can be used in pulsed electric fields of $1.5 \mathrm{MV} / \mathrm{m}$ and have less than $10 \%$ variation in resistance [8]. The resistivity of the electrolyte solution is a function of temperature, but the single shot nature of these experiments reduced the variability in resistance to negligible levels. The resistance, $\mathrm{R}[\Omega]$, of an aqueous-electrolyte resistor is given by Equation 3.2 where $l[\mathrm{~m}]$ is the length of the resistor, $A\left[\mathrm{~m}^{2}\right]$ is the electrode area, and $\rho[\Omega \mathrm{m}]$ is the resistivity of the solution which is a function of the concentration of the electrolyte in solution [8].

$$
R=\frac{\rho l}{A}
$$

Three aqueous resistors were placed in parallel to form an equivalent resistance of less than $10 \Omega$. Each of these resistors had a minimal length of approximately $0.07 \mathrm{~m}$, a fixed electrode area of $5 * 10^{-4} \mathrm{~m}^{2}$, and the same concentration of electrolyte solution. Sodium thiosulfate was used as the electrolyte because it has a lower resistivity than the more typical electrolyte, copper sulfate.

These resistors were cleaned and their electrolyte changed as a matter of circuit maintenance every 3-4 months. The final equivalent resistance of the three resistor network is typically between 5.0- $6.5 \Omega$. 


\section{3 Plasma Test Fixture Geometry}

The plasma circuit drives a 40 AWG silver-plated copper wire causing the wire to explode and form the initial plasma discharge. The test fixture, shown in Figure 3.10 and Figure 3.11 , is $10.16^{*} 10^{-2} \mathrm{~m}(4 ")$ thick polycarbonate with a $1.27^{*} 10^{-2} \mathrm{~m}(1 / 2$ ") diameter hole. The walls of the hole constrict the radial expansion of the exploding wire plasma discharge. Two lengths of $40 \mathrm{AWG}$ wire connect the brass anode with the stainless steel cathode screen.

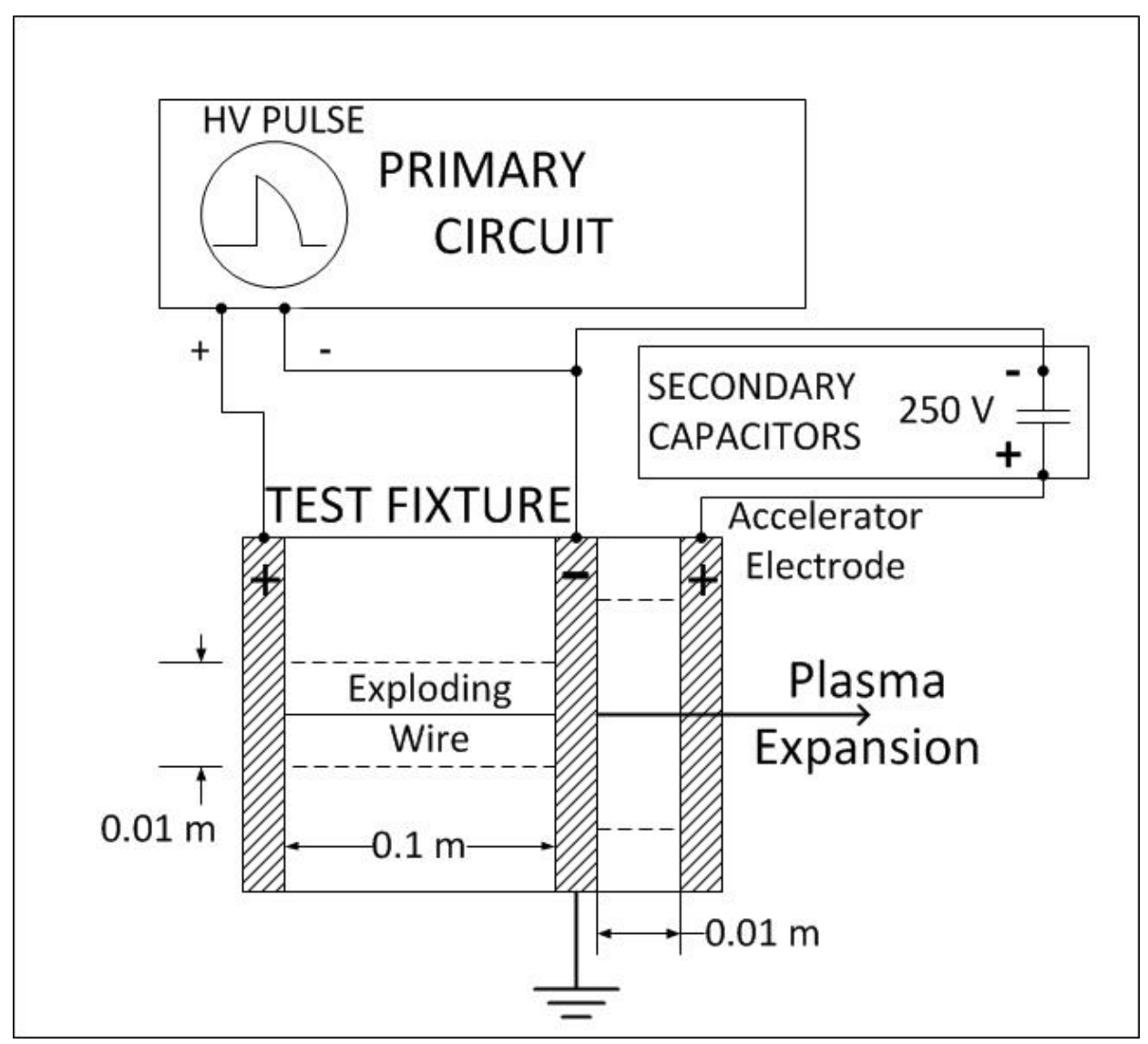

Figure 3.10 Toroidal air plasma fixture geometry

A $1.27 * 10^{-2} \mathrm{~m}$ thick piece of plastic separates the cathode screen from the accelerator grid. This spacer has a $2.54 * 10^{-2} \mathrm{~m}$ diameter hole centered on the tube housing the wire to allow for the plasma to expand and travel through both screens. During expansion, when the area between the cathode screen and the accelerator screen is filled with 
plasma, the secondary capacitors discharge their stored energy through the plasma. The 300 A plasma current provided by the secondary capacitors has a pulse width of approximately $4 \mathrm{~ms}$. The bulk of the TAP exits the accelerator region, detaching from a stationary discharge between the screens. After the bulk of the plasma has separated, the capacitors continue to discharge through the remaining plasma in the accelerator region. The plasma generation test stand is described in further detail in Appendix D along with CAD drawings.

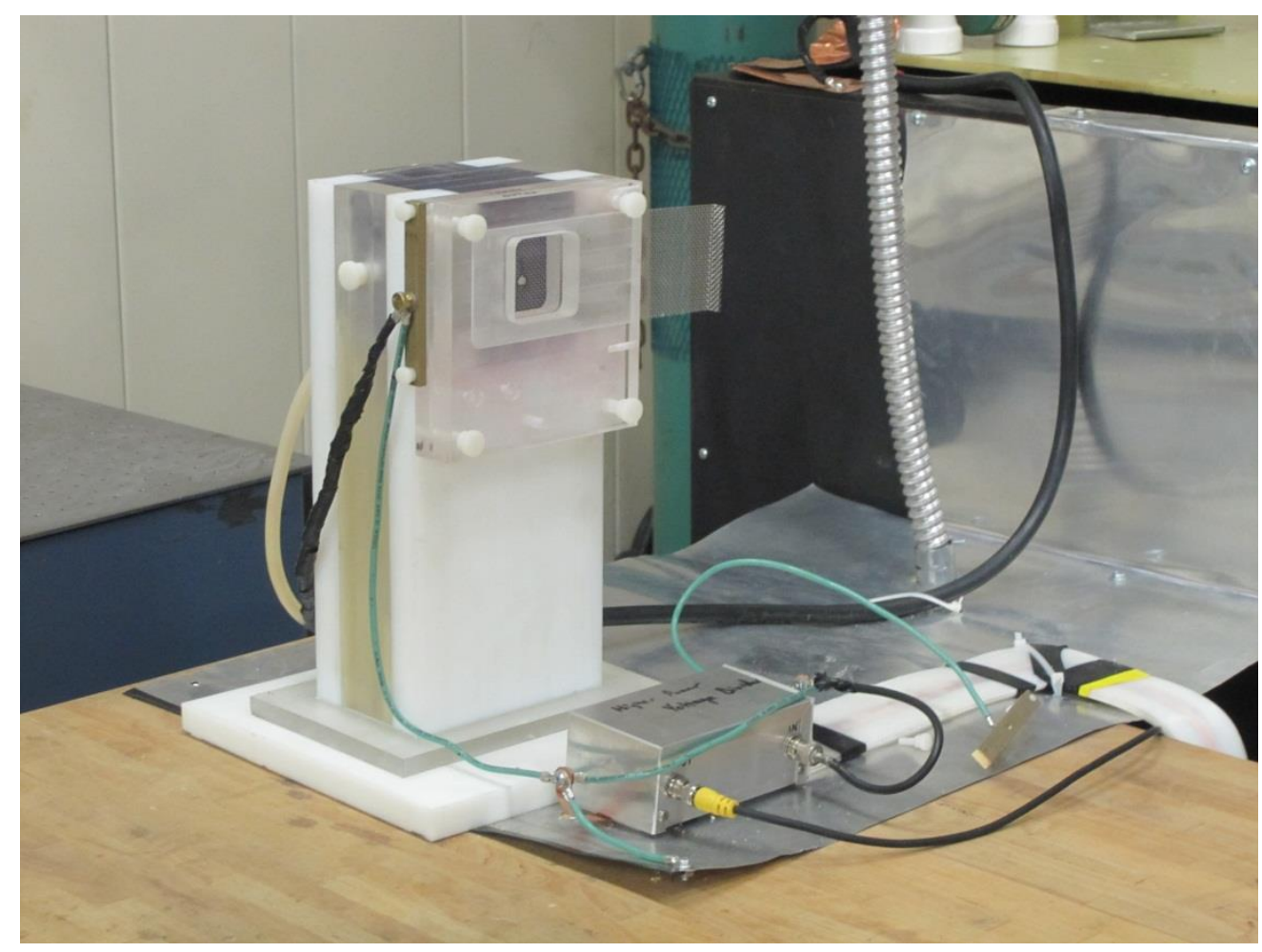

Figure 3.11 Toroidal air plasma fixture assembly

\section{4 Accelerator Circuit}

An accelerator circuit was designed to impart energy into the plasma in its early stages. The energy from these capacitors is discharged directly into the plasma once the plasma fills the area between two screens: the high voltage cathode from the plasma circuit, and the accelerator grid. As will be described in Chapter 5, this additional energy 
has proven to enhance the size and duration of the plasma and is critical in forming the toroidal shape.

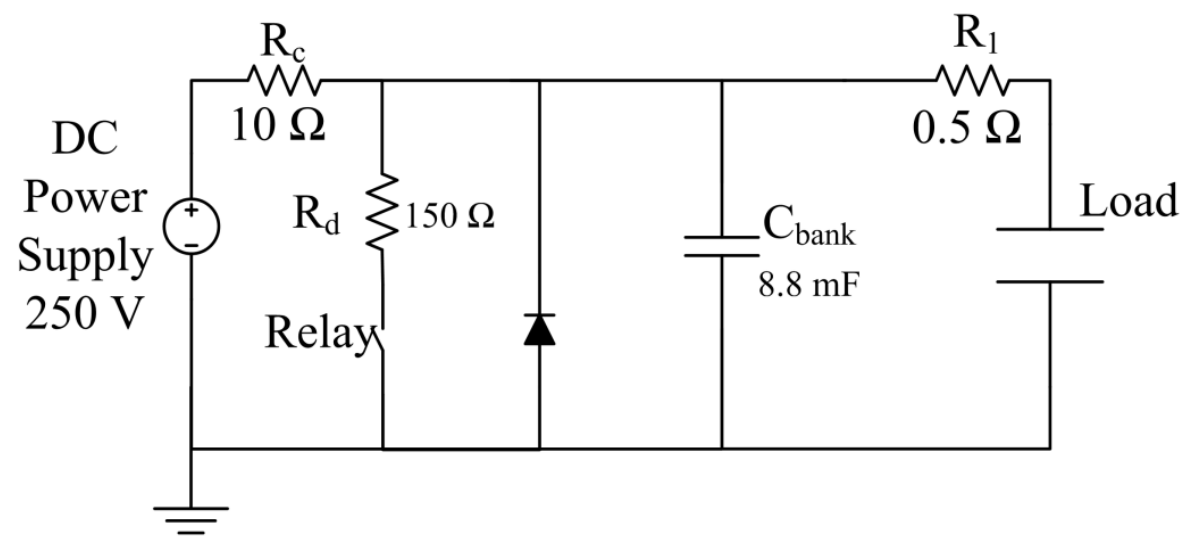

Figure 3.12 Accelerator circuit diagram

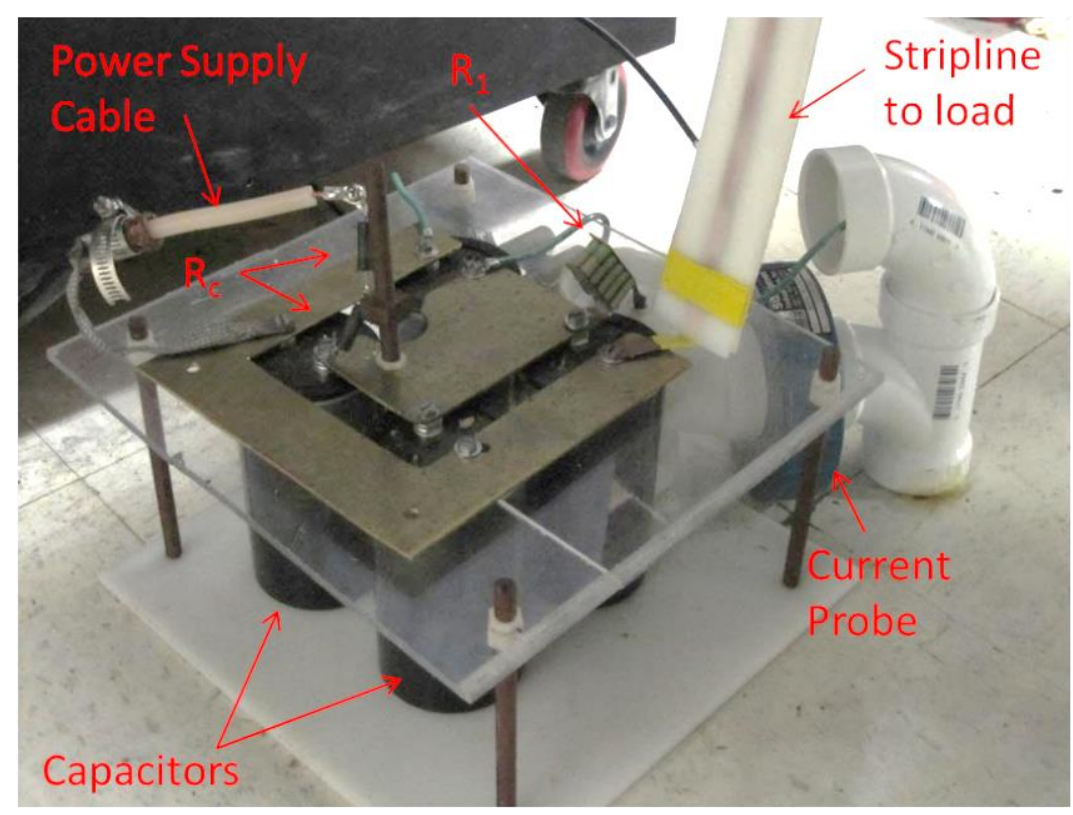

Figure 3.13 Accelerator circuit

The accelerator circuit, shown in Figure 3.12 and Figure 3.13, consists of four electrolytic capacitors totaling $8.8 \mathrm{mF}$, and a load resistor of $0.5 \Omega$ in series with two stainless steel screens held approximately $0.016 \mathrm{~m}$ apart by the plasma fixture geometry. The capacitors are charged to $250 \mathrm{~V}$ through a $10 \Omega$ resistor by a Powerten, Inc. 
constant-current, constant-voltage Power Supply (Model No. 163C-30033). The power supply is protected from reverse currents by a HVR-1X3 high voltage diode. The accelerator circuit is housed as close as possible to the plasma fixture and is connected to the plasma fixture by a copper strip transmission line to minimize stray inductance. This

was done to ensure fast delivery of the stored energy into the plasma. The accelerator circuit is also equipped with a shorting relay designed to handle a fully charged discharge through a $150 \Omega$ resistor to ground.

\section{5 Exploding-Wire-Triggered Shock Tube}

Shock tubes are a common and well-studied method of producing shock waves [9-11]. A shock tube typically consists of two sections separated by a diaphragm. When the diaphragm ruptures, the pressurized gas from the driver section expands into the lower pressure driven section and propagates down the shock tube forming a shock wave [9-11]. For this research rupture of the diaphragm was achieved by driving a large current pulse through a thin wire attached to the diaphragm, exploding the wire and sending the shock wave down the tube and out the open exit of the driven end.

The driver section was pressurized to $210 \mathrm{kPa}$ (30.5 psi), and the diaphragm was ruptured by driving a large, fast-rising current pulse through a thin copper wire, $9.8 * 10^{-2} \mathrm{~m}$ in length attached to the diaphragm. This vaporized the wire as shown in Figure 3.14, which triggered a failure of the diaphragm and sent a shock wave down the tube. The $210 \mathrm{kPa}$ operating pressure was chosen based on the results of a series of experiments which tested different operating pressures on a single $0.127 \mathrm{~mm}$ thick diaphragm. The conclusion from these experiments was that $210 \mathrm{kPa}$ was the maximum pressure possible which did not lead to self-rupture (from over pressure), but was able to 
rupture the diaphragm with the exploding wire. For timing considerations of the interaction experiments, it was critical that the rupture of the diaphragm be triggered by the exploding wire, and not self-rupture. Additional details on the triggered rupture of the shock wave are given in [12].

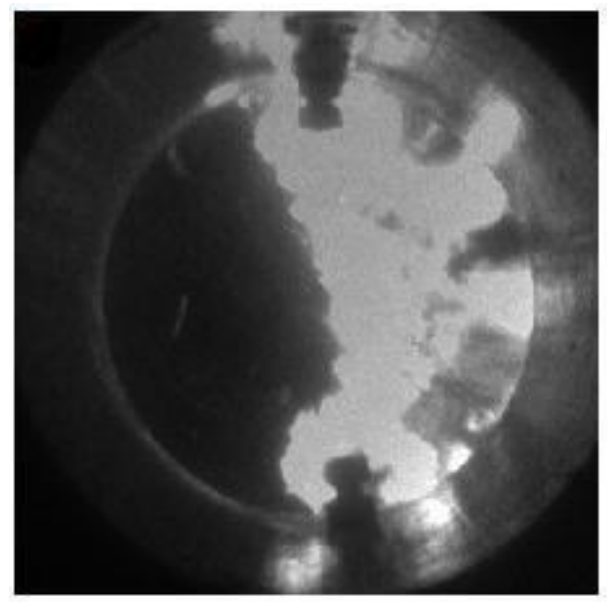

Figure 3.14 Discharge from exploding wire which ruptures the diaphragm

A $0.2 \mathrm{~m}(8$ ") schedule-80 PVC shock tube was assembled in the laboratory for the purpose of generating a robust and reliable shock wave. As shown in Figure 3.15, the tube is divided into two sections: a $0.61 \mathrm{~m}(2 \mathrm{ft})$ driver section and a $1.52 \mathrm{~m}(5 \mathrm{ft})$ driven section. Flanges were mounted on each section for coupling. We used a $1.27 * 10^{-4} \mathrm{~m}$ (5 mil) thick aluminized mylar film as the diaphragm material separating the two sections. A single $1.27 * 10^{-4} \mathrm{~m}$ thick diaphragm under a slow pressure rise self-ruptures near $275 \mathrm{kPa}$ (40 psig). A gas inlet was installed in the tube, along with a digital pressure gauge to monitor the pressure during the experiments. Two high voltage leads were added to the driver for the electrical connections for the exploding wire.

On the driven section, two Kistler 603B1 pressure transducers were mounted flush with the inner wall to measure the pressure changes traveling along the tube after the diaphragm ruptures. The sensors were mounted $0.33 \mathrm{~m}$ apart and $0.89 \mathrm{~m}$ from the 
diaphragm as shown in Figure 3.15. A picture of the shock tube is shown in Figure 3.16. A similar construction of this shock tube was previously presented in [13].

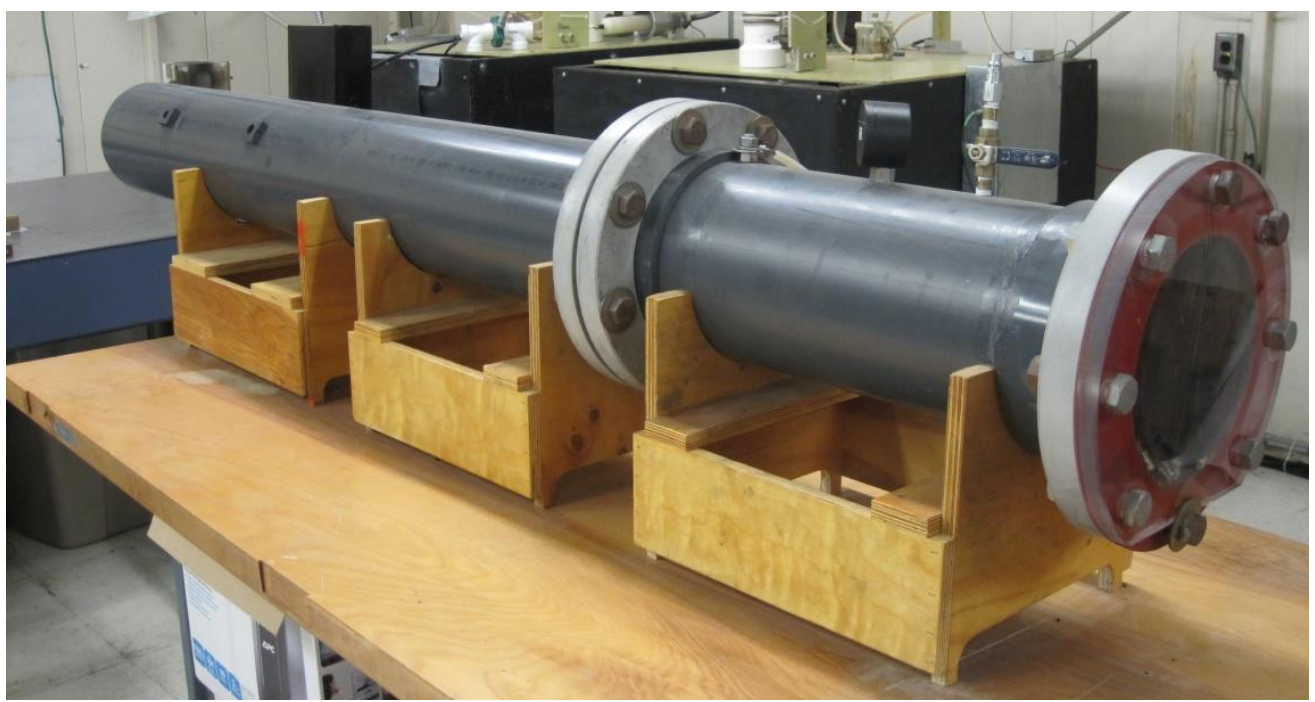

Figure 3.15 Shock tube assembled for the experiments

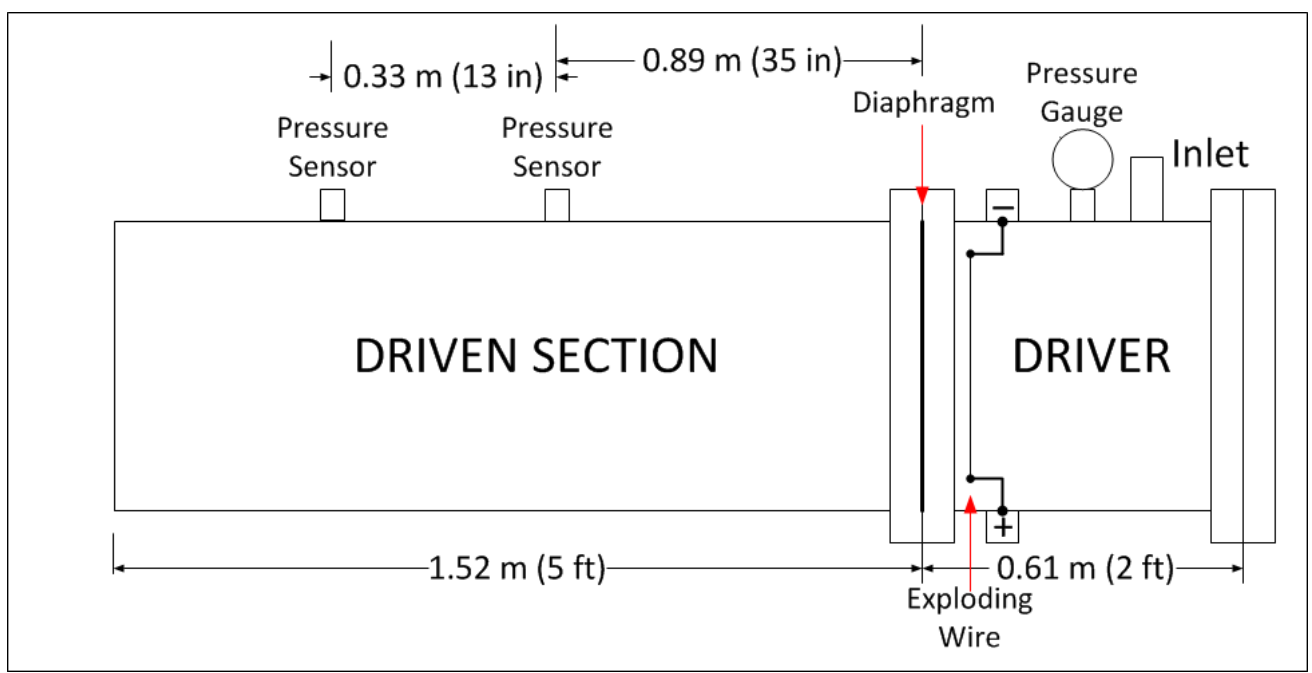

Figure 3.16 Diagram of shock tube with dimensions

\section{6 Shock Tube Circuit}

The shock tube circuit, shown in Figure 3.17, is similar in topology to the plasma circuit. Again, the primary components are the capacitor bank, the power supply and its components for protection, a three-plate pressurized gas switch, an aqueous-electrolyte resistor, the air-core inductor, and the load. 
The capacitor bank and spark gap switch are identical to the ones employed in the plasma circuit. The shock tube circuit is charged with a Varian Power Supply which has a negative polarity. Thus, the capacitor bank in the shock tube circuit is charged to $-30 \mathrm{kV}$ with respect to the ground and the protection circuit polarity is reversed when compared to the plasma circuit.

The circuit was designed to rupture the diaphragm effectively by the quick expansion of the exploding wire. The air-core inductor in the circuit is $0.6 \mu \mathrm{H}$, and the aqueouselectrolyte resistor network was set to approximately $5 \Omega$. The trigger wire is a $9.8 * 10^{-2} \mathrm{~m}$ long, $30 \mathrm{AWG}$ copper wire attached to the diaphragm. These elements were sized to facilitate a fast current rise through the exploding wire, consistently rupturing the diaphragm from the wire burst, approximately $5 \mu$ s after switch closure.

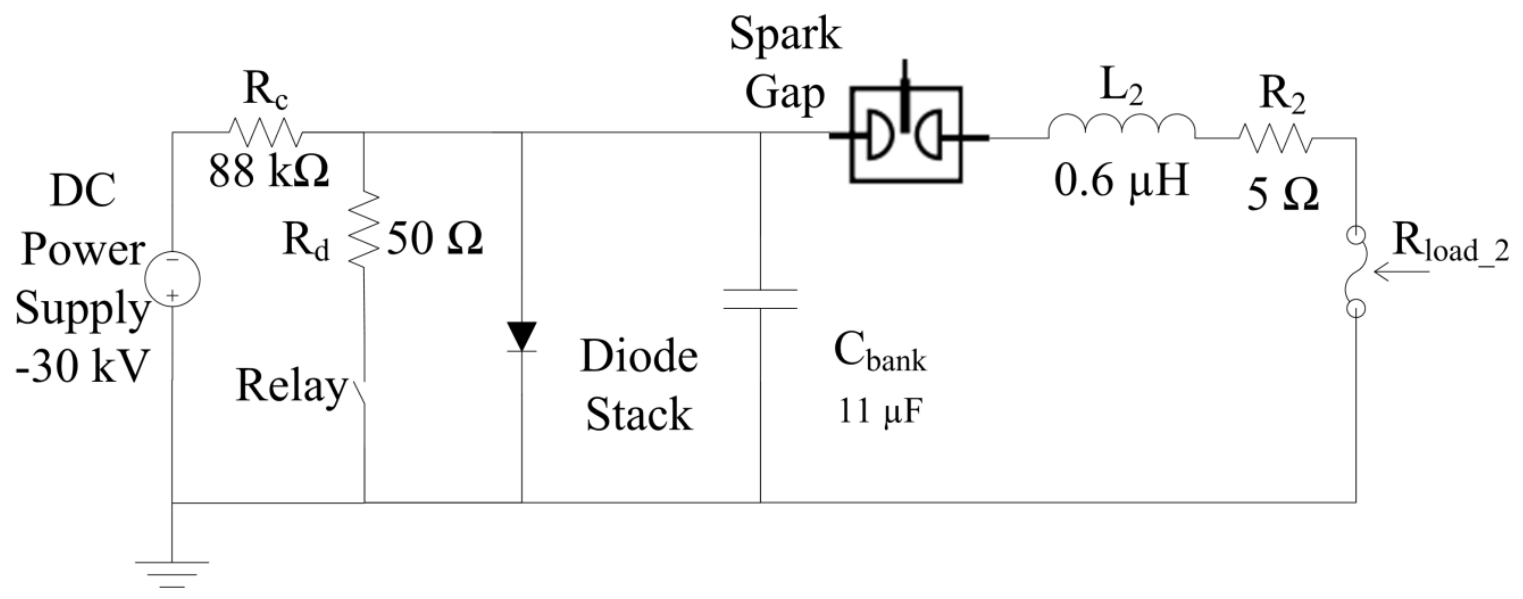

Figure 3.17 Shock tube circuit

The trigger jitter of the system in forming the shock wave is of critical importance to the experimental investigation. If the timing jitter is too high, the arrival of the shock wave within a pre-defined, fully diagnosed region in space (the exit of the tube), would be too inconsistent, and interaction results would not be reproducible. The target system jitter was $1 \mathrm{~ms}$ to reliably interact with a multi-millisecond duration air plasma with a 
shock wave. This was the primary advantage of rupturing the diaphragm with an exploding wire. The shock tube circuit was designed with the minimal trigger jitter of $32 \mu \mathrm{s}$. The full characterization of the timing jitter was measured by the pressure sensors equipped in the shock tube and is discussed in Chapter 4.

\section{7 Triggering and Control System}

The system which triggered each event in this experiment has been designed to minimize system jitter. Both the circuit and the shock tube circuit were, by design, triggered in the same manner. The following is a description for the shock tube circuit, but the same methodology is applied to the plasma circuit. This system is shown as a block diagram in Figure 3.18.

First, the spark gap switch is pressurized to 13 psi with compressed air. Then, the capacitor bank is charged slowly to $30 \mathrm{kV}$. The spark gap is designed to hold off this voltage until it receives a high voltage pulse from the trigger circuit, disturbing the electric field within the switch. At the push of a button on the digital delay generator, a +TTL pulse is sent into the control box triggering a fiber optic circuit that transmits a light pulse through a $10 \mathrm{~m}$ fiber optic cable. This pulse of light is received by a fiber optic receiver circuit, which immediately sends a $+\mathrm{TTL}$ pulse to the high voltage trigger circuit (described in Appendix B). Within the high voltage triggering circuit, the MSD 6420 capacitive discharge ignition system creates a medium voltage $(\sim 400 \mathrm{~V})$ pulse, which is stepped up to $40 \mathrm{kV}$ by an ignition coil and delivered to the midplane of the spark gap switch. The high voltage pulse on the third plate enhances the electric field in the spark gap to trigger breakdown of the switch, at which time the switch transfers the energy stored in the capacitor bank through the circuit inductor and into the dynamic wire load. 
The practical switching time from the manual trigger on the delay generator to the high voltage trigger for the spark gap is approximately $2,150 \mu \mathrm{s}$. In the case of the shock tube circuit, the exploding wire ruptures the diaphragm within the shock tube, releasing the pressurized air in the driver section. The pressurized air expands down the shock tube, forming a planar wave and exiting into the open air.

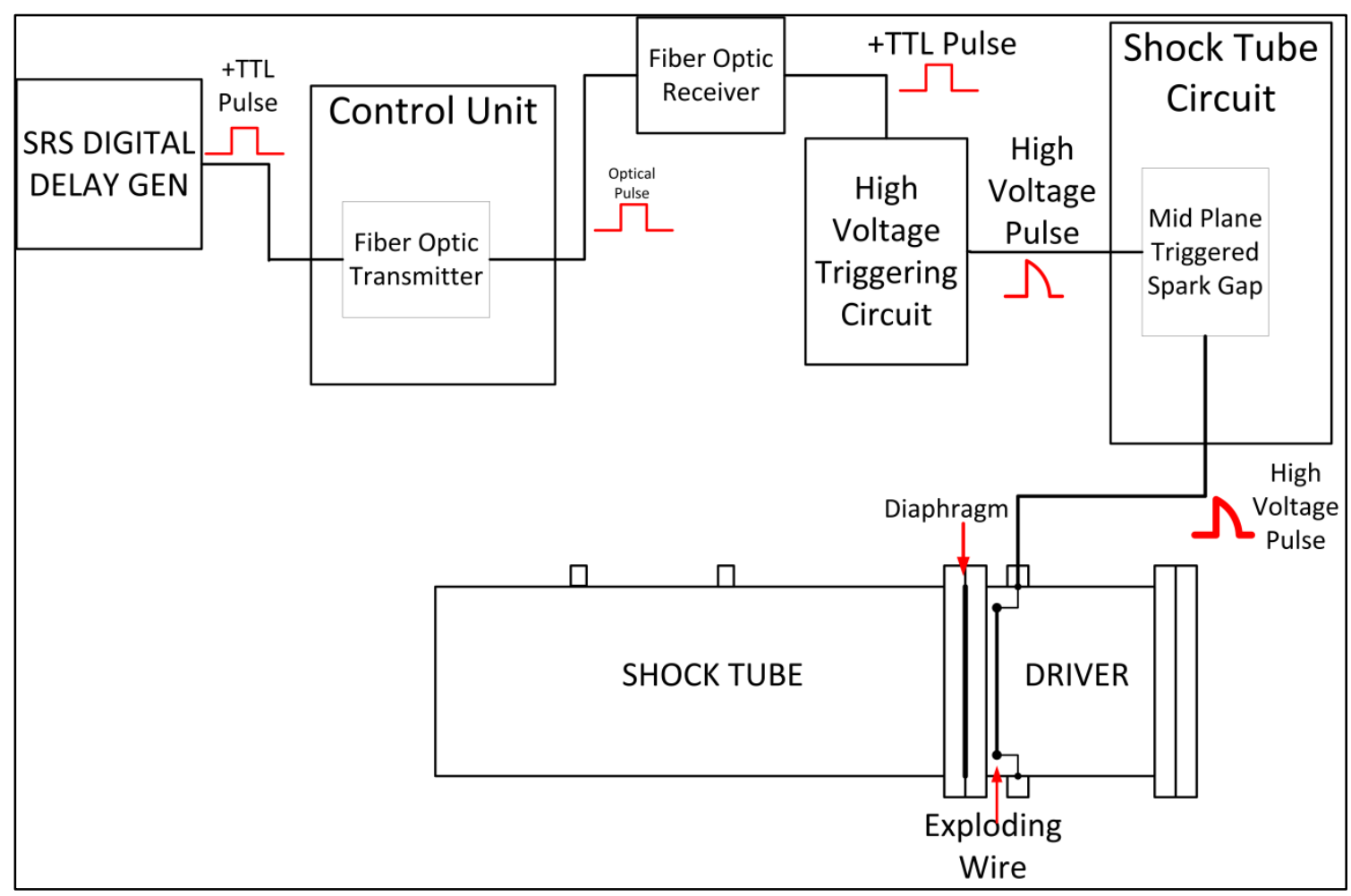

Figure 3.18 Block diagram of triggering system

For the plasma circuit, the wire vaporizes, expands, and forms the TAP (more details given in Chapter 5), and then travels toward the shock tube. The formation and transit time of the plasma is not the same as the formation and transit time of the shock wave. Thus, these two events cannot be triggered simultaneously. Independent triggering systems were used to trigger the shock tube circuit and plasma circuit. The SRS delay generator allows for any arbitrary delay between firing the circuits with 5 ps resolution. In practice, the shock wave transit time was longer than the formation time of the plasma, 
so the signal which ultimately triggers the plasma circuit was sent $2 \mathrm{~ms}$ after the signal which ruptures the diaphragm.

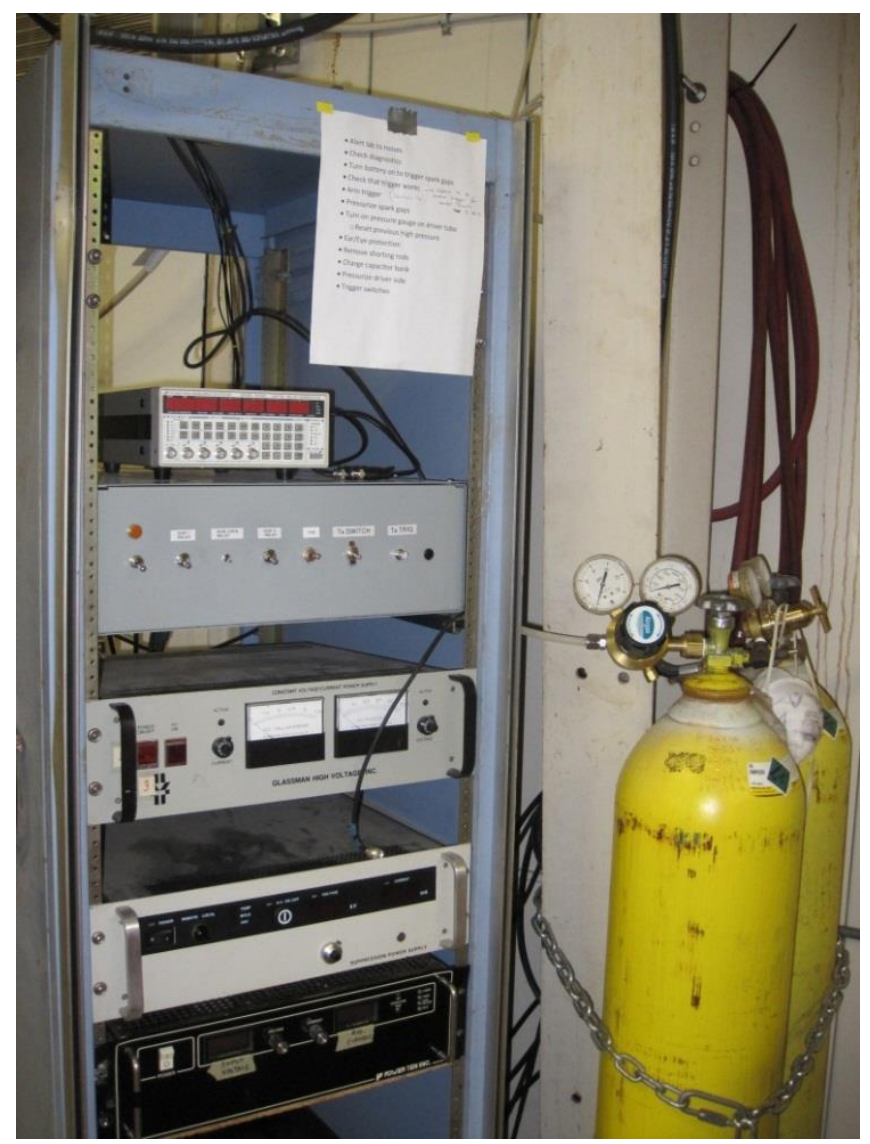

Figure 3.19 Control unit

For safe and reliable operation, the circuits and diagnostic suite were operated from a centralized location. This control unit, shown in Figure 3.19, consists of several rackmounted power supplies necessary to charge the high voltage capacitors, two compressed air cylinders used to pressurize the spark gap switches and the shock tube, a digital delay generator, and a switch box containing the optical trigger transmitter circuits and controls for the circuit shorting relays. The control unit is the focal point during the experiments and was designed for the choice of one of three experimental configurations: firing the plasma source only, firing the shock tube only, or firing both at a desired timing. 
To minimize RF interference, all trigger signals of the system are optical and are transmitted through fibers across the room to the various circuit components and diagnostics. Fiber optic $\mathrm{Tx} / \mathrm{Rx}$ circuits manufactured by Loree Engineering, which convert a +TTL pulse to a light pulse (for transmission) and vice versa (for receiving), are utilized. Each receiver circuit is contained within a small shielded stainless steel enclosure. These Rx circuits were spread around the system to control the trigger of each diagnostic. The control unit is the hub for six synchronous transmitter signals. The SRS DG645 digital delay generator allows for the precise timing and control of sending as many as 4 separate TTL signals. Further explanation of the timing and control of the experiments is given in Appendix B along with a timing diagram.

\section{References for Chapter 3}

1. K. A. O'Connor, "Compact Power Conditioning and RF Systems for a High Power RF Source," Ms. Thesis, Elec. and Comp. Engr., University of Missouri, Columbia, MO, 2008.

2. Hong Tang and Scuka, V., "The Breakdown Mechanism of a Mid-Plane Triggered Spark Gap Trigatron," Dielectrics \& Elect. Insulation, vol. 3, no. 6, pp. 843-848, 1996.

3. D. B. Hopkins, "Design Considerations and Data for Gas-Insulated High Voltage Structures", Symp Eng. Problems of Fusion Research, San Diego, CA, November 1821, 1975.

4. J.D. Cobine, Gaseous Conductors: Theory and Engineering Applications, McGrawHill Book Company, Inc., New York and London, 1941.

5. E. Husain and R. S. Nema, "Analysis of Paschen Vurves for Air, N2, and SF6 using the Townsend Breakdown Equation," Trans. Elect. Insulation, vol. EI-17, no. 4, 1982.

6. 'Series T-670 High Precision Spark Gap Switches,' L-3 Communications Pulse Sciences., San Leandro, CA, 2002.

7. J. Dams, et al., "Computer Aided Design of Three Electrode Spark Gaps", 4th Int. Symp. on HV, pp. 541-544, 1983.

8. R.E. Beverly III and Associates 'Application Notes for Aqueous Electrolyte Resistors'. [Online]. Available: http://www.reb3.com/pdf/r_appl.pdf. (accessed 2011).

9. Dr. Genick Bar-Meir, 'Fundamentals of Compressible Flow' [online]. Available: http://www.potto.org/gasDynamics/node117.php, 2007 
10. University of Purdue, "Shock Tube Background," [online] Accessed: 2011. Available:

https://engineering.purdue.edu/AAE/Academics/Courses/aae3341/Shock\%20Tube/Sh ock\%20Tube\%20Background.docx.

11. K. A. Smith, "Characterization of Transient Pressure Loads in the Reservoir of a Hypersonic Blowdown Tunnel," M.S. thesis, Dept of Aero. Engr., University of Maryland, College Park, 2005.

12. M E.J. Rudroff, et al., "The Generation of Triggered Shock Waves in Shock Tubes with Exploding Wires," Pulsed Power Conf., pp.1072-1076, 2011.

13. A. Lodes, et al., "The Interaction of Shock Waves with High Density Air Plasma," Pulsed Power Conf., pp.1077-1080, 2011. 


\section{CHAPTER 4: DIAGNOSTICS}

The experiments are measured with a diagnostic suite including current and voltage probes, high speed cameras, a single-mirror Schlieren system, and pressure transducers. This suite has been used to characterize the plasma and shock wave independently as well as during interaction. In addition to this equipment, a spectroscopy system has been used to analyze the spectral emission of the plasma discharge.

\subsection{Circuit Diagnostics}

Both the plasma circuit and the shock tube circuit, are monitored during experiments with voltage and current probes. Many different nodes of each circuit have been monitored to ensure proper operation. A typical configuration monitored the voltage across and current through the wire load of the plasma circuit as well as the voltage across the wire load of the shock tube.

A Tektronix TDS 2024C oscilloscope was used to capture and monitor the data from the voltage and current probes. It operates with a $200 \mathrm{MHz}$ bandwidth, a sample rate of $2 \mathrm{GS} / \mathrm{s}$, a 2500 point record length, and a fifth external input [1]. The oscilloscope has four channels and is triggered by sending a + TTL signal to the fifth input (EXT IN). When additional channels are required, a Tektronix DPO 7254 oscilloscope was used. It operates with a $2.5 \mathrm{GHz}$ bandwidth, a maximum sample rate of $20 \mathrm{GS} / \mathrm{s}$, and a maximum record length of $12,500,000$ points[2].

A Northstar High Voltage PVM-6 probe, shown at the left of Figure 4.1, featuring 1000x attenuation was used to monitor the voltage across the wire load. This probe is capable of measuring a maximum DC voltage of $60 \mathrm{kV}$, or a pulsed voltage of $100 \mathrm{kV}$ at a maximum frequency of $80 \mathrm{MHz}$ [3]. Figure 4.2 is the differential voltage across the 
plasma-generating exploding wire measured with two PVM-6 Northstar High Voltage probes. The voltage across the exploding wire rises from $0 \mathrm{~V}$ to more than $40 \mathrm{kV}$ in $2.4 \mu \mathrm{s}$ and begins to rapidly decay. As shown, the signal rings during the rapid decrease as the probe is not able to recover quickly enough to measure the signal with high accuracy.

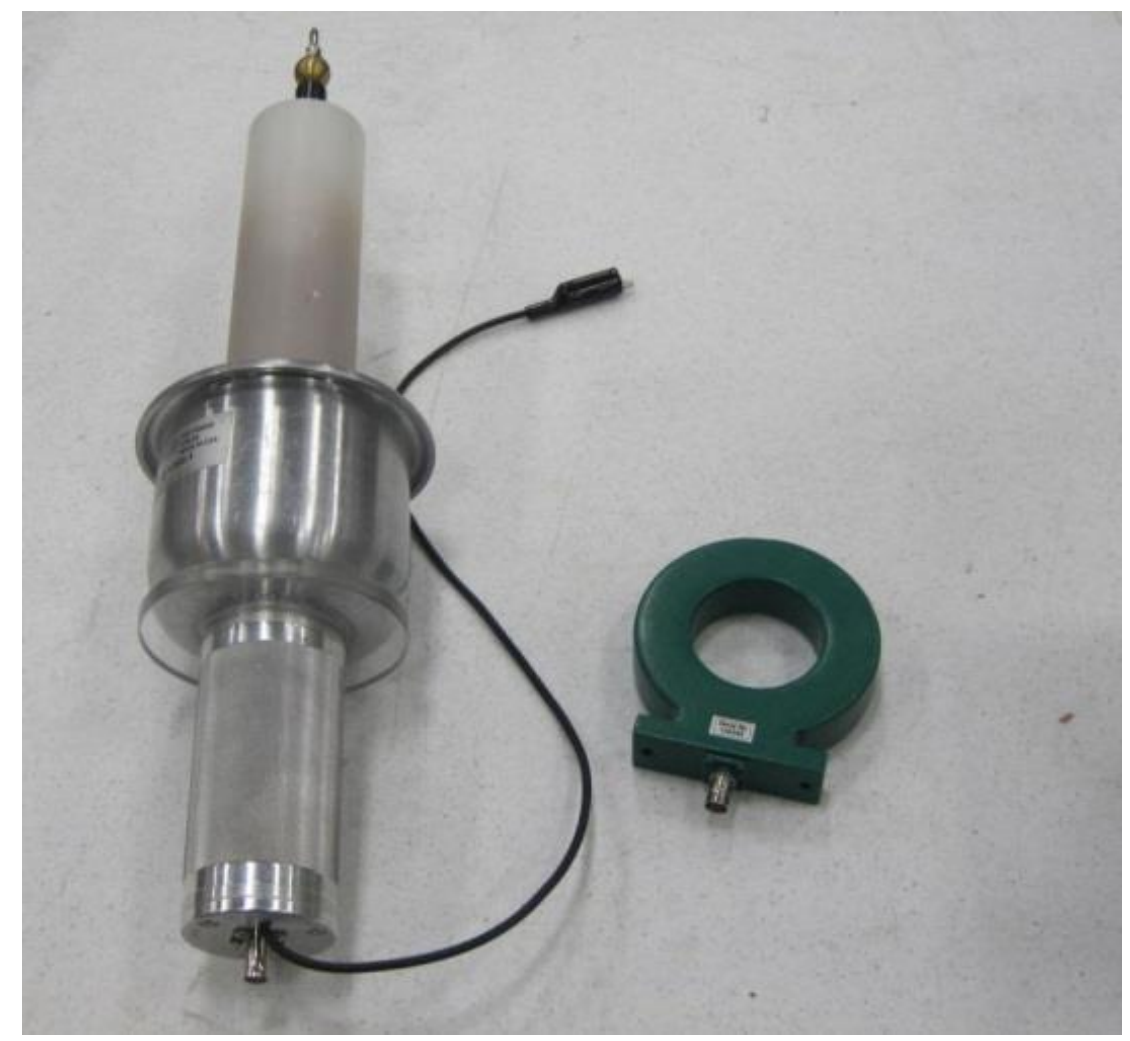

Figure 4.1 Circuit diagnostics, Northstar high voltage probe (left), Pearson current probe (right)

Figure 4.3 shows a typical experimental waveform of the voltage pulse applied to the exploding wire in the shock tube as measured by a Northstar High Voltage PVM-6 probe. The voltage reaches a peak of $-24.1 \mathrm{kV}$, with a re-strike occurring at approximately $11 \mu \mathrm{s}$ after switch closure. 


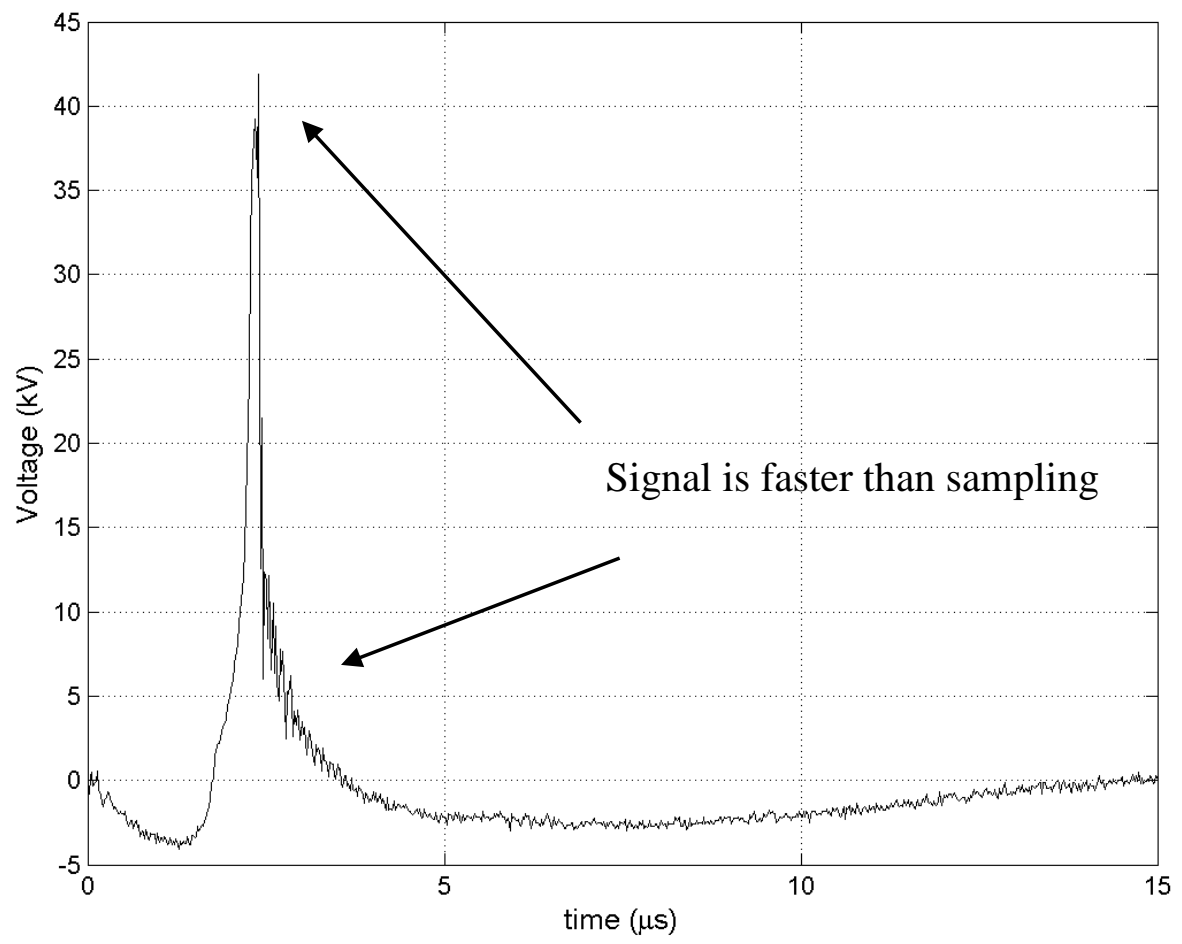

Figure 4.2 Voltage across the exploding wire of the plasma circuit

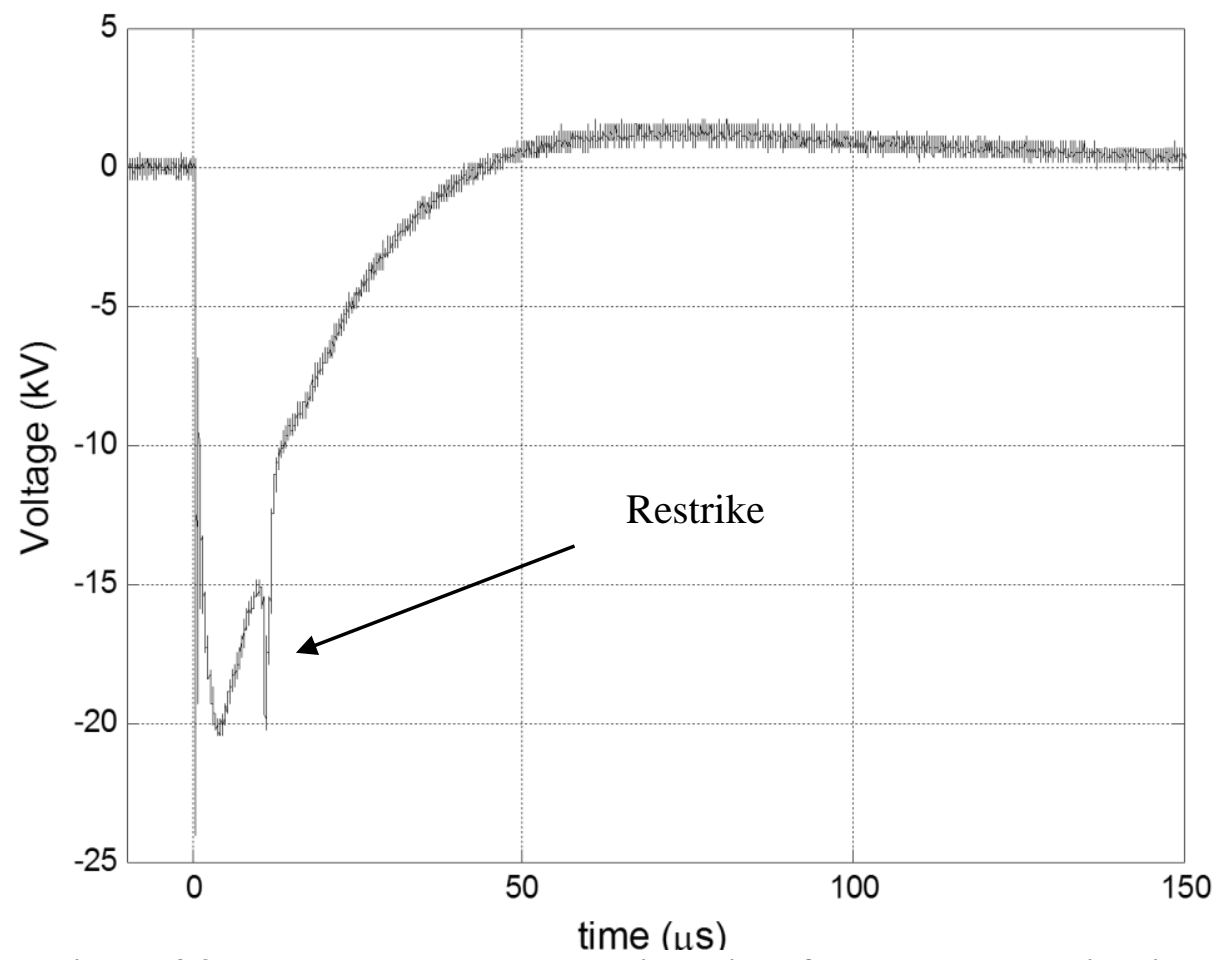

Figure 4.3 Voltage across the exploding wire of the shock tube circuit 
A custom made voltage divider, shown in Figure 4.4, is used to measure the voltage between the accelerator screens. This diagnostic was designed to withstand the large current and high power of the accelerator circuit by utilizing ten parallel high-power ceramic Caddock resistors, each rated for $2 \mathrm{~W}$ of average power. The final voltage division ratio is $117.5: 1$. The input leads are connected across the accelerator circuit screens, and the output leads are connected to an oscilloscope via a $50 \mathrm{Ohm}$ BNC cable. The circuit is mounted in an aluminum housing to minimize RF interference.

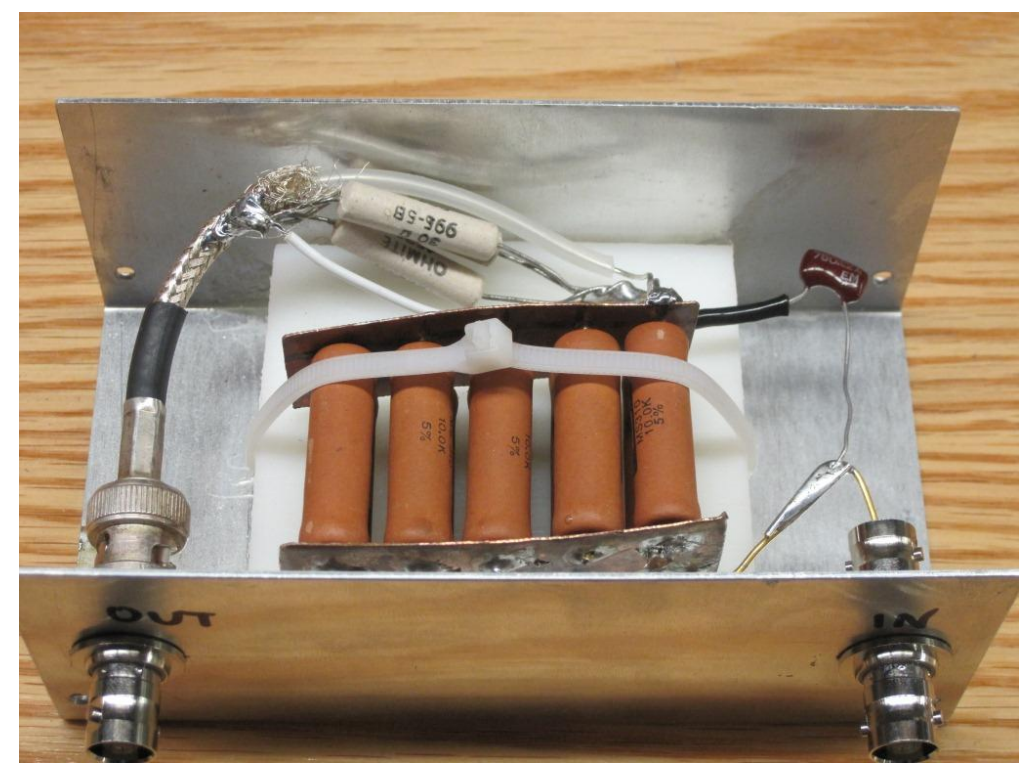

Figure 4.4 Voltage divider used to observe the voltage across the accelerator screens

Figure 4.5 is a typical voltage waveform from this diagnostic. The diagnostic is heavily influenced by noise in the initial stages of wire vaporization, but at approximately $18.6 \mu \mathrm{s}$, the voltage drops to $50-100 \mathrm{~V}$ and maintains those levels for over a millisecond. This is a good measure of the amount of time necessary for the exploding wire to expand and fill the accelerator region. 


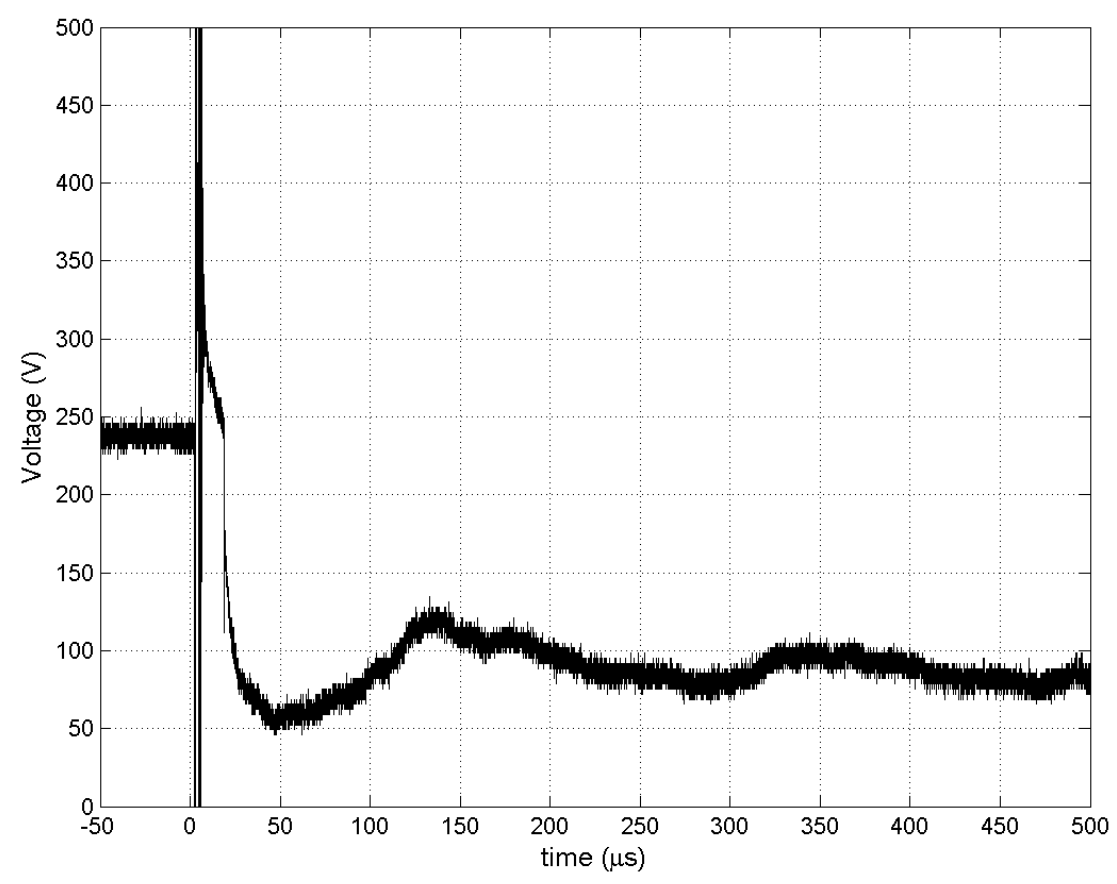

Figure 4.5 Voltage across accelerator screens

A Pearson Electronics 101 current probe, shown in the right of Figure 4.1, is used to measure the current through the exploding wire. It is capable of measuring a peak current of $50 \mathrm{kA}$ with a sensitivity of $0.01 \mathrm{~V} / \mathrm{A}$ and a useable rise time of $100 \mathrm{~ns}$ [4]. It is connected to the high impedance terminal of the oscilloscope with a $20 \mathrm{~dB}$ attenuator and a $50 \Omega$ terminator.

Figure 4.6 is the current through the exploding wire of the plasma circuit as measured by the Pearson 101 Current Probe. The current rises from $0-6.16 \mathrm{kA}$ in $11.6 \mu$ s with a 10- $90 \%$ rise time of $6.9 \mu \mathrm{s}$ and a $45 \mu$ s pulse width.

A Stangenes SI-5009 current probe is also utilized in these experiments, which has a maximum measurable current of $200 \mathrm{kA}$ and a $0.001 \mathrm{~V} / \mathrm{A}$ sensitivity [5]. Figure 4.7 shows a typical experimental waveform of the current through the exploding wire of the shock tube circuit measured by the Stangenes SI-5009 current probe, with a peak current of $6.76 \mathrm{kA}$ and confirming re-strike at approximately $11 \mu \mathrm{s}$ after switch closure. 


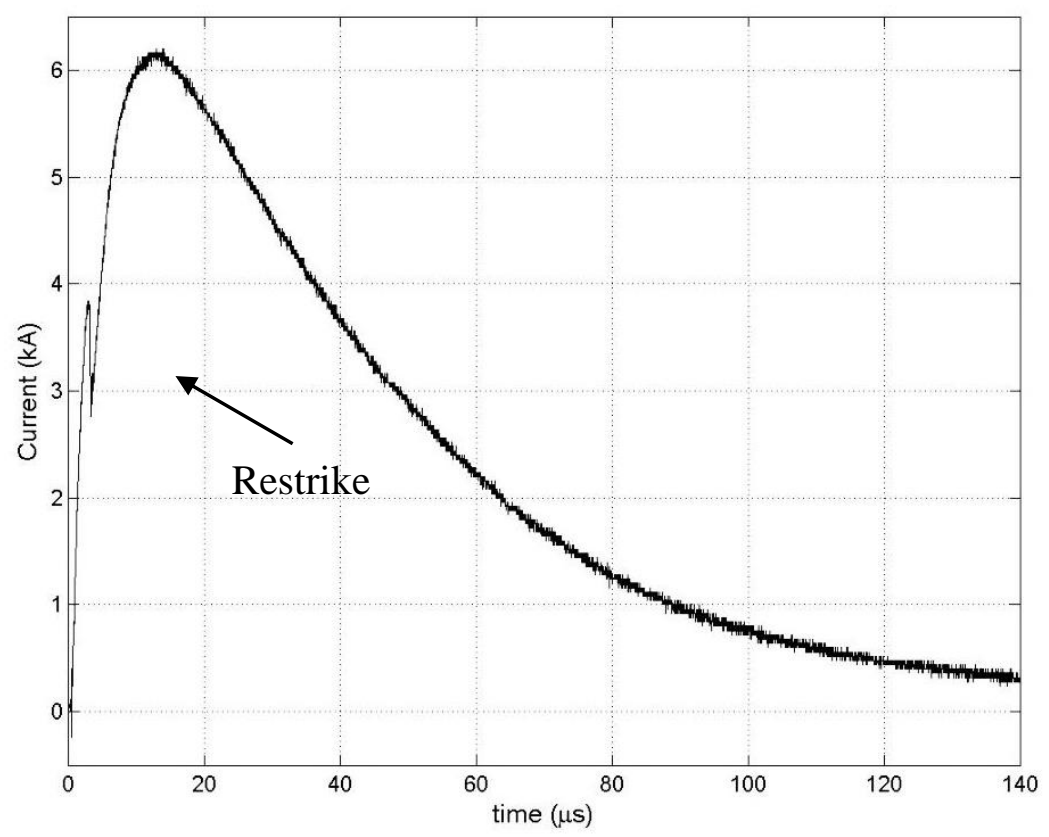

Figure 4.6 Current through the exploding wire of the plasma circuit

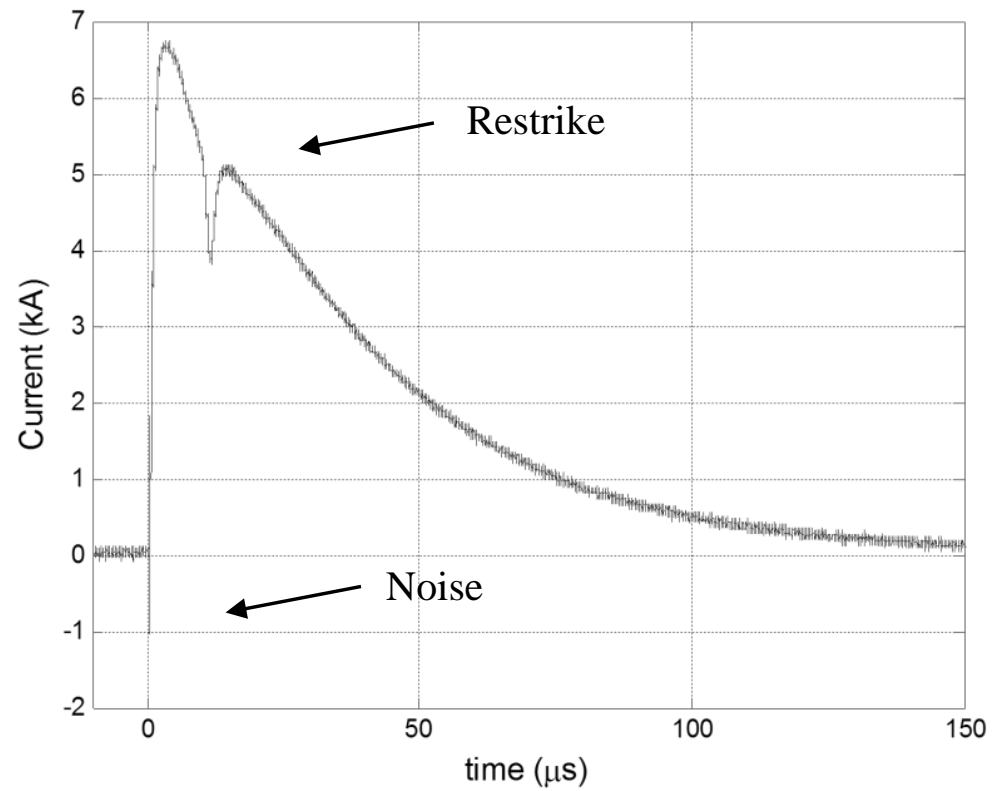

Figure 4.7 Current through the exploding wire of the shock tube circuit

Figure 4.8 is the current flowing between the accelerator screens as measured by a Stangenes SI-5009 Current Probe. After an initial period of noise, the current of the accelerator screen rises up to a maximum of $536 \mathrm{~A}$ and stays above $300 \mathrm{~A}$ for over $1 \mathrm{~ms}$. 


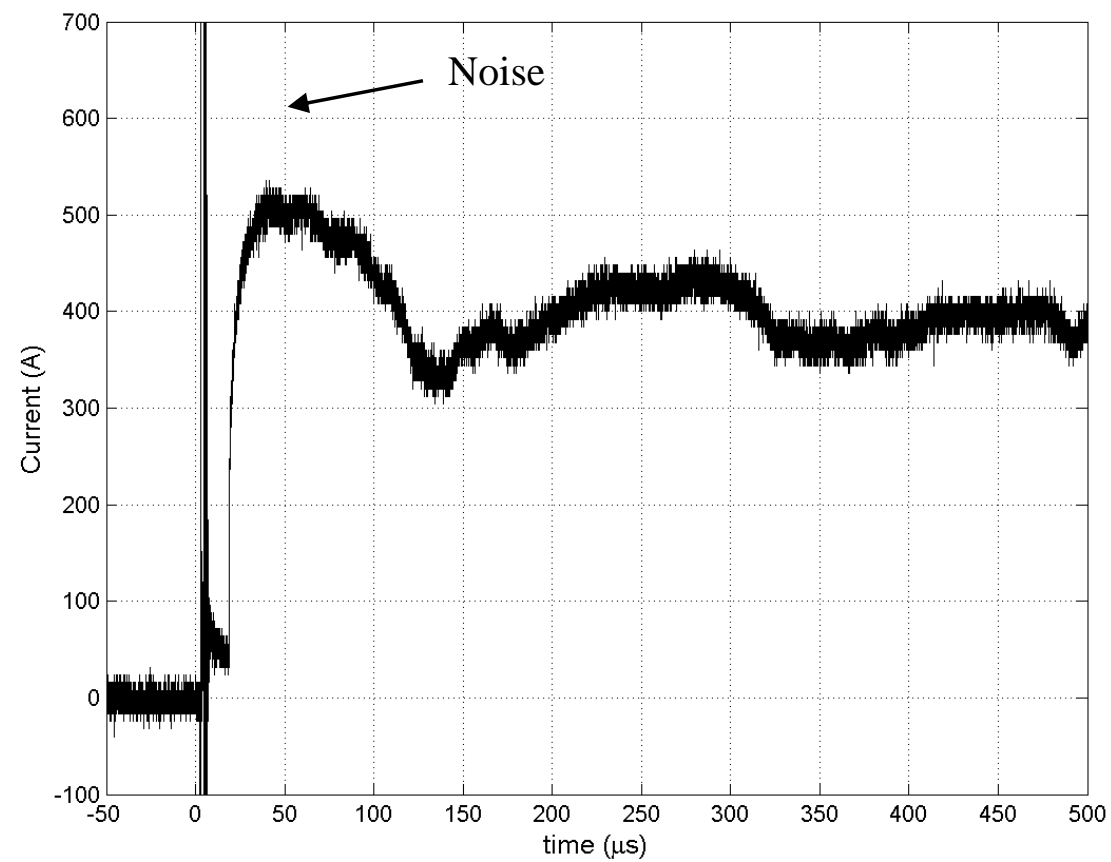

Figure 4.8 Current delivered by the accelerator circuit

\subsection{High Speed Cameras}

Three high speed cameras were employed throughout these experiments at UMC: a Vision Research Phantom v7.3, an SVSI GigaView, and a DRS Ultra 8, shown from left to right in Figure 4.9.

The Phantom v7.3 has a maximum resolution of $800 \times 600$, a 6,688 fps frame rate, a minimum shutter speed of $2 \mu \mathrm{s}$, and a maximum recorded file size of $8 \mathrm{~GB}$. At reduced resolution, the v7.3 can image up to $222,222 \mathrm{fps}$. The sensor is 14-bit monochrome with a $22 * 10^{-6} \mathrm{~m}$ pixel size. The typical settings employed by UMC are $512 \times 256$ resolution, with a $21,052 \mathrm{fps}$ frame rate and a $2 \mu$ s exposure. The camera was typically configured to keep a continual buffer of recorded frames until a trigger is received, after which, a set number of post-trigger frames are recorded. Total maximum record time with these settings is over 1.4 seconds [6]. 

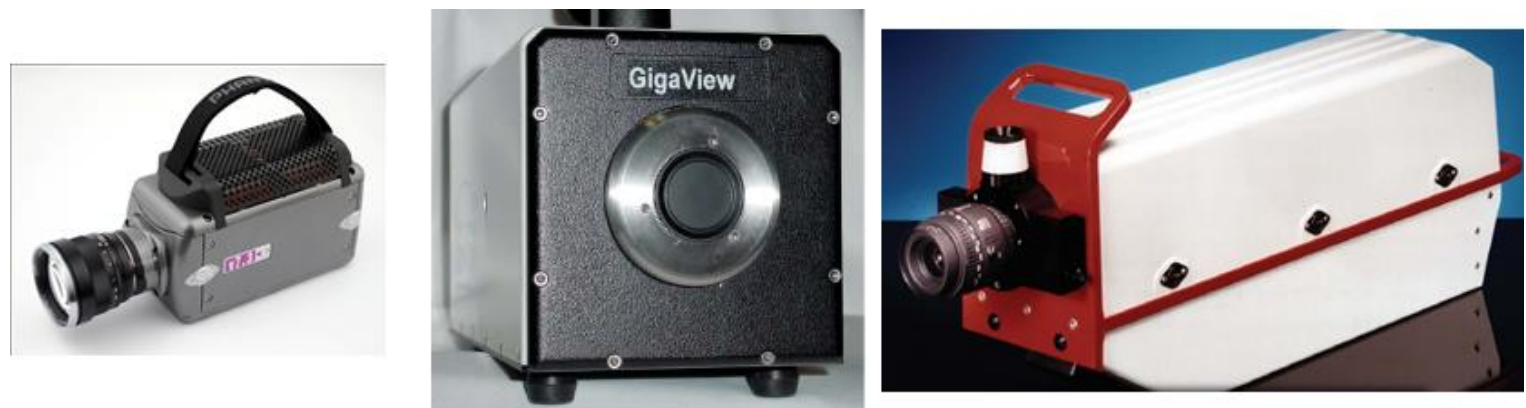

Figure 4.9 High speed cameras at UMC, Phantom v 7.3 (left), SVSI GigaView (center), and DRS Ultra 8 (right).

The SVSI GigaView high speed camera has a maximum resolution of 1280 x 1024 with $65 \mathrm{fps}$ and a minimum shutter speed of $2 \mu \mathrm{s}$. At reduced resolutions, the maximum frame rate of the GigaView is 4,000 fps. The sensor is 10-bit monochrome with a $12 \times 12^{*} 10^{-6} \mathrm{~m}$ pixel size. UMC typically configured this camera at $320 \times 256$ for a 2,130 frame rate with a $9 \mu$ s exposure over a total record time of $0.1 \mathrm{sec}$ [7].

The DRS Ultra 8 is used for capturing higher speed events. The Ultra 8 is capable of capturing up to $100,000,000 \mathrm{fps}$ for a maximum of eight frames. Each frame has a 520 x 520 resolution with a 12-bit range. Each frame operates on a separate channel, and the camera may be configured to capture any sequence of 8-frames at any custom timing diagram. The Ultra 8 has an inherent 100 ns delay with a range of exposure times and interframe times of $10 \mathrm{~ns}-1 \mathrm{~ms}[8]$.

\subsection{Image Processing Analysis of the TAP}

For further analysis of the high speed images of the TAP, a program was created in MATLAB® to track the size and shape of the plasma discharge as it propagates. This program is presented in detail with implementation in Appendix C, but several details of the algorithm must be discussed in this section to understand the analysis.

A normal torus (donut) is typically described by a major radius, $\mathrm{R}$, and a minor radius, $r$. The shape is formed by revolving the small circle of radius $r$, around the larger 
circle of radius $\mathrm{R}$. Rather than using the traditional minor and major radii, the video analysis describes the TAP by its height and width from a side axis perspective. These dimensions are shown in Figure 4.10. These dimensions are used for convenience when the camera is positioned in the side axis perspective. The algorithm has been modified to characterize the minor and major radii of the TAP for the experiments in which the camera is in the on-axis perspective (Section 5.3.5).

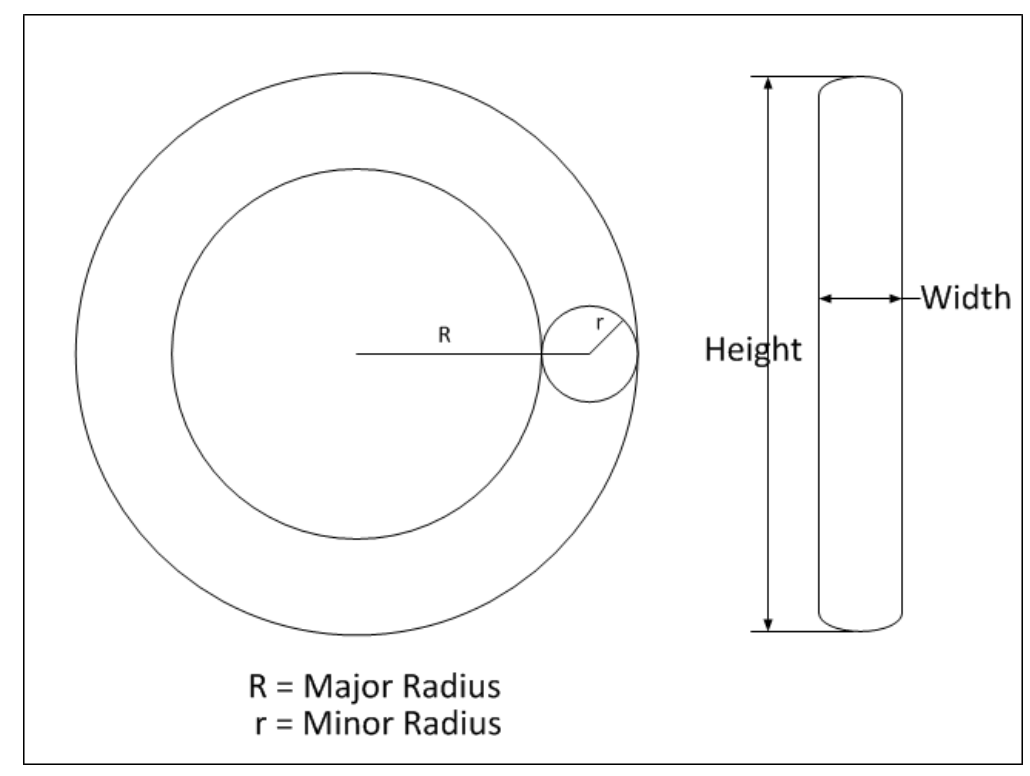

Figure 4.10 Toroid dimensions of the image processing analysis of the TAP

The program was written to track the height, width, area, and distance traveled of the TAP throughout its lifetime. The algorithm was designed to analyze the formation and evolution of the TAP from a side-on view. The program calculates each of these four functions for every frame recorded by the Phantom 7.3 camera. The algorithm for height calculates the longest column of pixels that are identified as plasma by a predetermined brightness threshold. The algorithm for width likewise calculates the widest row of pixels that are characterized as plasma. The algorithm to calculate the area of the plasma counts 
the number of illuminated pixels. Finally, the algorithm also tracks the distance travelled of the tallest column of pixels.

As each image is processed, these four calculated variables are stored in an array for plotting and analysis. These four variables are tracked as time evolves in each frame of a given video file. This allows for the direct comparison of separate experiments, which have separate videos.

Due to several basic assumptions and limitations of the image processing program, the results presented in Chapter 5 and Chapter 6 are approximations. The algorithm will calculate the results from the video for each execution, but the values calculated are smaller than those in the laboratory.

Additionally, due to blooming effects in the early frames of each video, the plasma is analyzed as larger in the opening frames than is realistic. This is an optical and digital effect due to the temporary saturation of the pixels of the CCD in the high speed camera. The plasma is brightest during its initial expansion, but the camera lens aperture settings were configured to record the size, shape, and duration of the TAP once it has detached from the plasma fixture. During these later stages, the discharge emits light at a lower intensity, and thus the camera lens was configured for low light environments. During the intense emission of the early stages of the plasma discharge, the CCD pixels saturate, thus the data calculated from the early portions of each video are inaccurate, and the first $750 \mu \mathrm{s}$ of the data is omitted. The reader is referred to Appendix C for the details on the implementation of these algorithms and a discussion on sources of inaccuracy. 


\subsection{Schlieren Imaging}

Schlieren imaging is an effective method of producing qualitative images of refractive-index gradient fields $[9,10]$. Changes in the refractive index of a gas are typically due to density changes, temperature variations, or changes in flow [9]. These refractions can be visualized through Schlieren techniques.

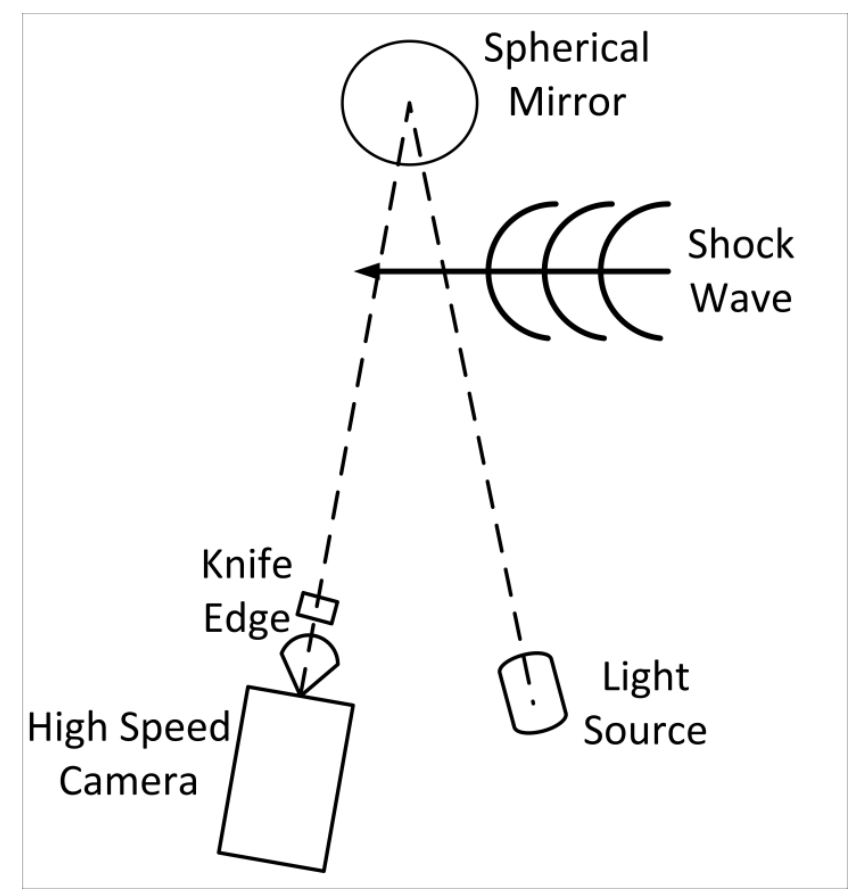

Figure 4.11 Schlieren system at UMC

A single mirror Schlieren system was added to the experimental test bench in the laboratory to observe the shock wave, the TAP discharge, and any changes to either resulting as time evolves. The Schlieren system, shown in Figure 4.11, has four major components: a single point light source, a $0.15 \mathrm{~m}$ spherical mirror, a knife edge, and a high speed camera.

The single-point light source in the system is created by passing the light from an ultra-bright red LED through a magnifying lens. The spherical mirror then collects the image of the light source and focuses it onto the razor blade knife edge. If any changes in 
the refractive index occur in the area between the light source and the mirror, the light focused on the knife-edge will be partially blocked, creating a visual effect that is observed by the high speed camera. This allows for the viewing of disturbances created by the plasma and the shock wave. Each component must be carefully aligned for optimum imaging. Thus, the system is fixed to several optical rails and is re-adjusted after every experiment. A typical shock wave front produced by this test bench is shown through a Schlieren image in Figure 4.12.

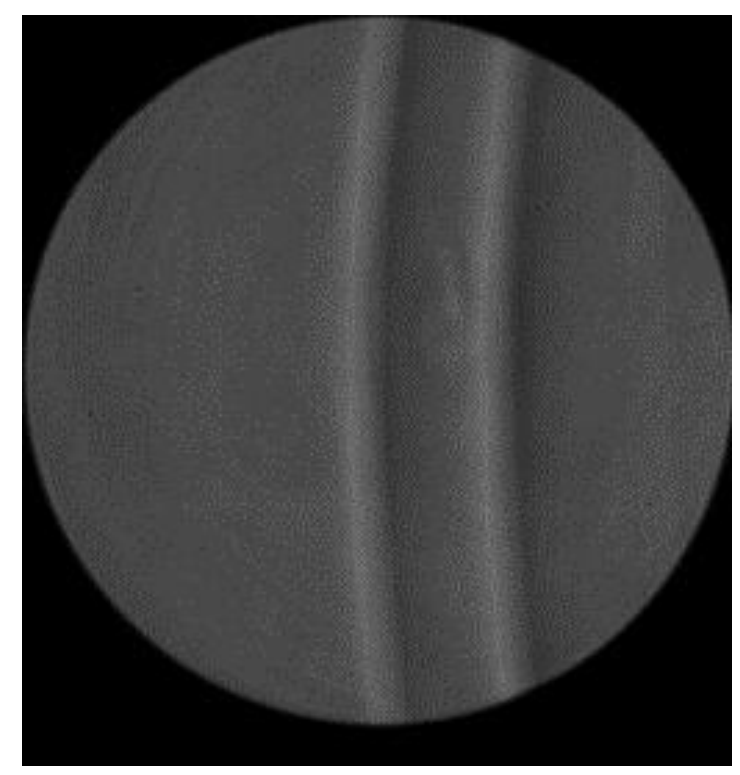

Figure 4.12 Schlieren image of the shock wave

\subsection{Pressure Diagnostics}

Pressure sensors were another useful diagnostic in these experiments. Two Kistler 603B1 charge based pressure transducers were utilized to characterize the shock wave propagating from the shock tube. The sensors have a $1 \mu$ s rise time and are calibrated for two pressure ranges ( $0-150 \mathrm{psi}$, and 0-1500 psi) and provide a linear response within $\pm 1 \%$ of the calibration. The Kistler sensors are conditioned by corresponding Kistler 5010B amplifiers, which are equipped with a $180 \mathrm{kHz}$ low pass noise filter [11, 12]. The 5010B 
amplifiers are single channel, outputting a voltage pulse proportional to a single sensor's calibrated pressure to an oscilloscope. Typical calibration settings of one channel are $10 \mathrm{psi} / \mathrm{V}$ or $20 \mathrm{psi} / \mathrm{V}$.

The pressure wave, as observed by the first pressure sensor at $0.3 \mathrm{~m}$ from the exit of the tube, has a peak value of approximately $10 \mathrm{psi}$, as shown in Figure 4.13. This is in good agreement with calculations presented in [13].

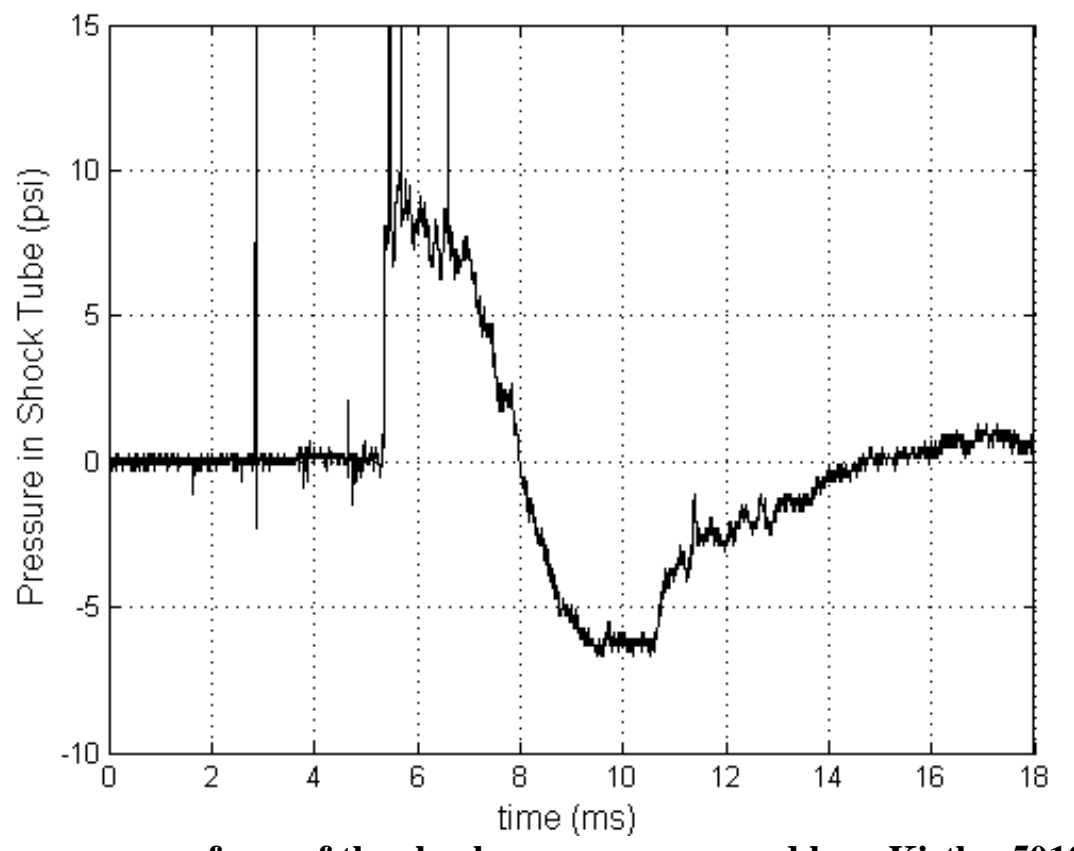

Figure 4.13 Pressure waveform of the shock wave as measured by a Kistler 5010B inside the tube $0.3 \mathrm{~m}$ from the exit

Numerous experiments were conducted to assess the practical timing jitter of the shock wave. The observed timing jitter of the entire shockwave production is $32 \mu \mathrm{s}$. A sample of four experimental results of the shock wave generation jitter tests are presented in Figure 4.14. These data account for the jitter of the whole system, from the positive TTL signal produced by the delay generator to the shock wave impulse on the pressure transducer located at the end of the shock tube. This delay is about $4,280 \mu$ s, as shown in Figure 4.14. 

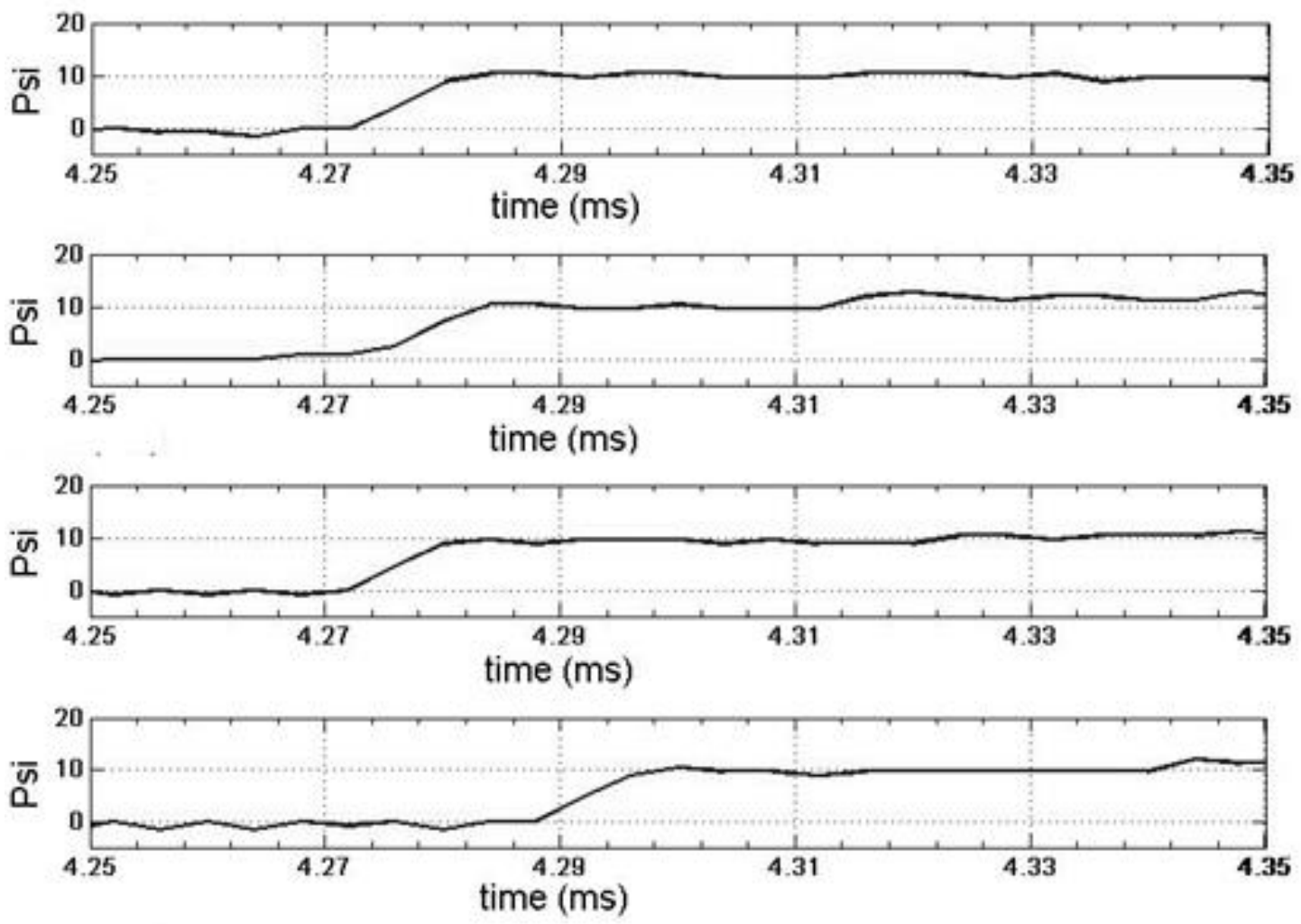

Figure 4.14 Arrival of shock wave front at the end of the tube, four trials are shown

Additionally, three Endevco piezoresistive pressure transducers have been utilized to accurately characterize the shock wave outside of the tube. The model $8530 \mathrm{C}-15$ pressure transducer is calibrated for the $0-15$ psi range and requires signal conditioning to facilitate maximum range and wideband frequency response. The Endevco model 136 three channel system was used for this purpose $[14,15]$. Like the Kistler system, the output of the three channels is connected via BNC cable to an oscilloscope.

These sensors were used to measure the shock wave outside of the shock tube at the front planar surface of the plasma test fixture during experiments with and without the plasma. The experiments, discussed in Chapter 8, required the lower pressure range of the Endevco 8530C-15 to accurately characterize the mitigation effects of the plasma on the shock wave. A typical shock wave front (without a plasma) $27.5^{*} 10^{-2} \mathrm{~m}$ from the shock 
tube exit as measured by one channel of the Endevco system is shown in Figure 4.15. As shown, the Endevco sensors, and to a lesser extent the Kistler sensors, are sensitive to noise from the RF emitted by the pulsed power circuits. A data smoothing algorithm, described in detail in Appendix E, is used to analyze the data more accurately. Additional details about the experimental setup are given in Chapter 8 along with the results.

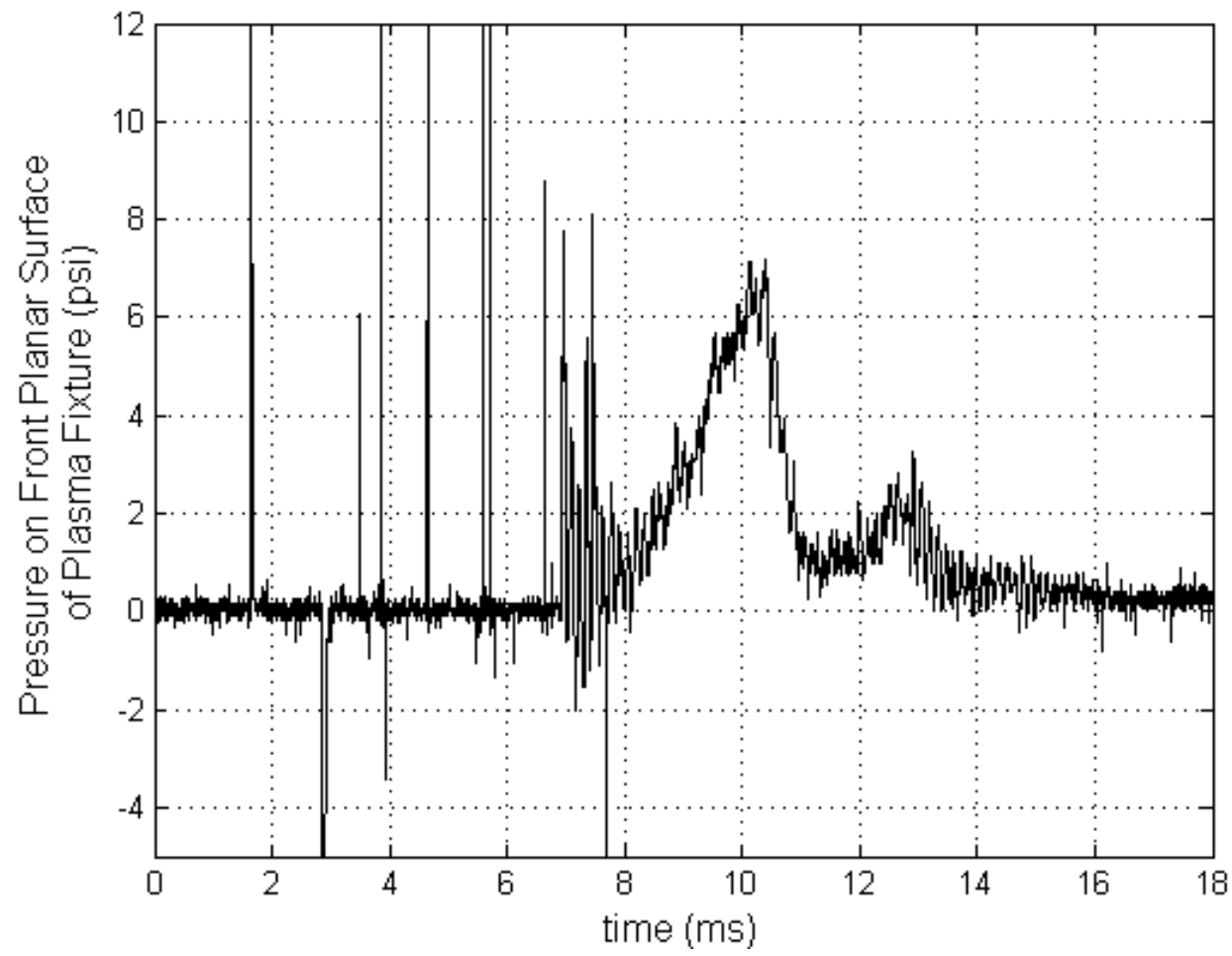

Figure 4.15 Pressure waveform of the shock wave as measured by an Endevco 8530C-15 mounted on the front planar surface of the plasma fixture (this trial is without plasma interaction).

\subsection{Spectroscopy System}

Another very useful diagnostic in observing and characterizing air plasmas is the measurement of the spectral emission. An optical multichannel analyzer was configured into a spectrographic system for the purpose of characterizing air plasma discharges. This diagnostic system consists of an intensified CCD camera mounted to a spectrograph. The entrance slit of the spectrometer is outfit with a SMA mount for a fiber optic cable for 
light collection. The system is controlled via a PC to collect the data. The spectrograph used is an Acton Research Corporation SpectraPro-300i, with three diffraction gratings. The intensified CCD attached to the Spectra Pro is a Princeton Instruments ICCD-MAX with a $1,024 \times 256$ pixel screen and a pixel size of $26 \times 26^{*} 10^{-6} \mathrm{~m}$. The timing and gating of the CCD is controlled by a ST-133 controller with a programmable timing generator. These components communicate and collect data onto a PC via the WINSPEC $®$ software. Light is collected via a $400 * 10^{-6} \mathrm{~m}$ quartz silica fiber optic cable. After the calibration procedure, described below, is performed, the system is able to efficiently observe a spectrum from $200-1,000 * 10^{-9} \mathrm{~m}$. This system is housed in a shielded enclosure to protect it from radio frequency (RF) electromagnetic emissions that result from firing the high voltage circuits.

Since the spectrograph system has varying efficiencies at different wavelengths, a two-step process is necessary for accurate calibration and data analysis. First, a wavelength calibration was performed in the WINSPEC software with a Newport 6035 mercury-argon pen lamp. This allows the software, the CCD, the spectrograph, and the controller to be spectrally in sync. The second step of calibration was to characterize the spectral efficiency of the system. Additionally, the CCD was removed, a flat field correction was performed, and the CCD was realigned with the spectrometer.

Each component of the system (e.g. the optical fiber, the diffraction grating, the CCD) has different spectral transmission and detection efficiency profiles. An experimental method of characterizing the total system spectral efficiency is to measure a known source and compare the results to the known emission profile of the source [16]. 
For this method, two standard spectral irradiance lamps with overlapping emission profiles were used.

The Newport 63945 deuterium lamp runs at $30 \mathrm{~W}$ with a spectral range of 200- $400 * 10^{-9} \mathrm{~m}$. The Newport model number 63355 quartz tungsten halogen (QTH) runs at $200 \mathrm{~W}$ and has a spectral irradiance range of $250-2,400 * 10^{-9} \mathrm{~m}$. Both lamps can be traced to NIST standards.

The system, including the optical fiber, was used to measure the emission of both the QTH lamp and the deuterium lamp. UMC used the emission data to calibrate the system and accurately measure the spectral emission of the plasma source. A secondary method of calibration for this system is also explored and analyzed in [17].

All data were exported from the WINSPEC $®$ software into MATLAB® where the necessary processing and correction of the data was performed. Processing of the spectrum involves correcting the data for both the flat field characterization and the calibration. This process accounts for the aperture limitations of the CCD-spectrograph mounting, and averages the five data points from the experimental repetition. The aperture of the mount vignettes the ICCD pixel range, limiting practical light collection. For the $3 * 10^{5}$ groove $/ \mathrm{m}$ diffraction grating blazed at $500 * 10^{-9} \mathrm{~m}$ used in these experiments, this limitation restricts practical light collection to spectral windows of about $200 * 10^{-9} \mathrm{~m}$. Because of this, data from outside the $200 * 10^{-9} \mathrm{~m}$ width for a given trial were discarded. Additionally, the vertical pixel columns of the CCD were summed for each wavelength.

The data processing procedure yields one intensity value per wavelength, with five data points for each wavelength from the experiment repetition. These five intensities 
were averaged and corrected against calibrated data as demonstrated by Equation 4.1.

The flatfield characterization and the lamp outputs and calibrations were normalized before being applied to the discharge emission data.

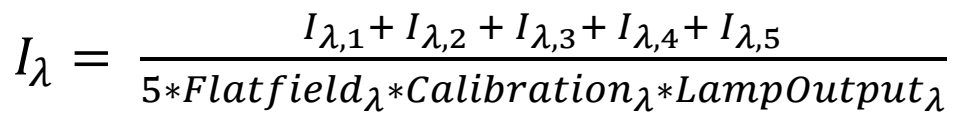

\section{References for Chapter 4}

1. Tektronix, Inc., "Digital Storage Oscilloscopes," TDS2000C series datasheet, 2010.

2. Tektronix, Inc., "Digital Phosphor Oscilloscopes," DPO7000 series datasheet, 2012.

3. North Star High Voltage, "PVM Series High Voltage Probes to $60 \mathrm{kV}$ DC," PVM-6 datasheet, [online] http://www.ppmpower.co.uk/high_voltage_probes_dividers/

4. Pearson, Inc., "Pearson Current Monitor Model 110A," 110A datasheet, Pearson Electronics, Inc., Palo Alto, CA 94303

5. Stangenes Industries Inc., "Current Transformers," Pulse Current Transformers, [online] http://www.stangenes.com/curtransformers.shtml

6. Vision Research, Inc., "Phantom v7.3," Vision Research, Inc., 2011. [Online]. Available: http://www.visionresearch.com/Products/High-Speed-Cameras/v73/

7. Southern Vision Systems, Inc., "GigaView Operator's Manual”, SVSI, Madison, AL 35758, Dec. 9, 2008.

8. DRS Technologies, "DRS's Ultra 8 High-Speed Imaging System," Ultra-8 datasheet, DRS Data \& Imaging Systems, Inc., Oakland, NJ 07436.

9. G.S. Settles, Schlieren and Shadowgraph Techniques, Spring-Verlag, 2001.

10. G.S. Settles, et al., "Full-scale Schlieren Flow Visualization," Flow Visualization VII, pp. 2-13, 1995.

11. Kistler Instrument Corporation, "Miniature, High-Frequency, Charge Output Pressure Sensors," Type 603 datasheet, Kistler Instrument Corporation, 2003, Amherst, NY 14228

12. Kistler Instrument Corporation, "Charge Amplifier," Type 5010B datasheet, Kistler Instrument Corporation, 2009, Amherst, NY 14228

13. M.E. J. Rudroff, et al., "The Generation of Triggered Shock-Waves in Shock-Tubes with Exploding Wires," Pulsed Power Conf., pp.1072-1076, 2011.

14. Endevco Corporation, "Piezoresistive Pressure Transducer," Model 8530C -15, -50, 100 datasheet, Endevco Corporation, San Juan Capistrano, CA 92675.

15. Endevco Corporation, "DC Amplifier," Model 136 datasheet, Endevco Corporation, San Juan Capistrano, CA 92675.

16. C. O. Laux, "Optical Diagnostics and Radiative Emision of Air Plasmas," PhD. Dissertation, Dept. Mech. Engr., Stanford University, 1993.

17. A. Lodes, et al., "Spectroscopic Measurements of a Toroidal Air Plasma" Pulsed Power Conf., pp.1277-1281, 2011. 


\section{CHAPTER 5: TOROIDAL AIR PLASMA DISCHARGE}

The toroidal air plasma (TAP) source was studied extensively with regard to volume, duration, and electron density. The primary discharge is formed in open air from a current-driven exploding wire and expands into a toroidal shape as it detaches from the apparatus. Images from the high speed cameras show temporal and spatial evolution of the discharge as it expands. The TAP is observed to be self-sustaining beyond approximately $10 \mathrm{~ms}$ while traveling $0.02 \mathrm{~m}$ or more from the test fixture. The toroidal ring expands to approximately $0.12 \mathrm{~m}$ in diameter. This chapter will discuss the typical formation, evolution, and characterization of the TAP.

\subsection{Formation and Evolution of the TAP}

Figure 5.1 is a sequence of high speed images from a typical discharge of the toroidal air plasma. The high speed video was recorded at $21,052 \mathrm{fps}$ with a period of $47.50 \mu \mathrm{s}$ by the Vision Research Phantom v7.3 using a $2 \mu \mathrm{s}$ exposure. The camera was aligned orthogonal to the plasma's direction of travel. These images shown in Figure 5.1 are $475 \mu \mathrm{s}$ apart. The first frame starts at 'first light' taken to be the point of wire vaporization. For the next $1.5 \mathrm{~ms}$, the discharge expands out in a mushroom shaped cloud similar to that formed in an explosion. At about $1.5 \mathrm{~ms}$ after vaporization, the plasma detaches from the assembly and continues its forward momentum away from the fixture. The plasma cloud further develops into a toroidal shape, as shown in the latter images of Figure 5.1 and Figure 5.3, and in the alternate video images shown in Figure 5.4. This toroid continues to travel away from the fixture, up to $0.39 \mathrm{~m}$, over an elapsed time of $12.4 \mathrm{~ms}$. 

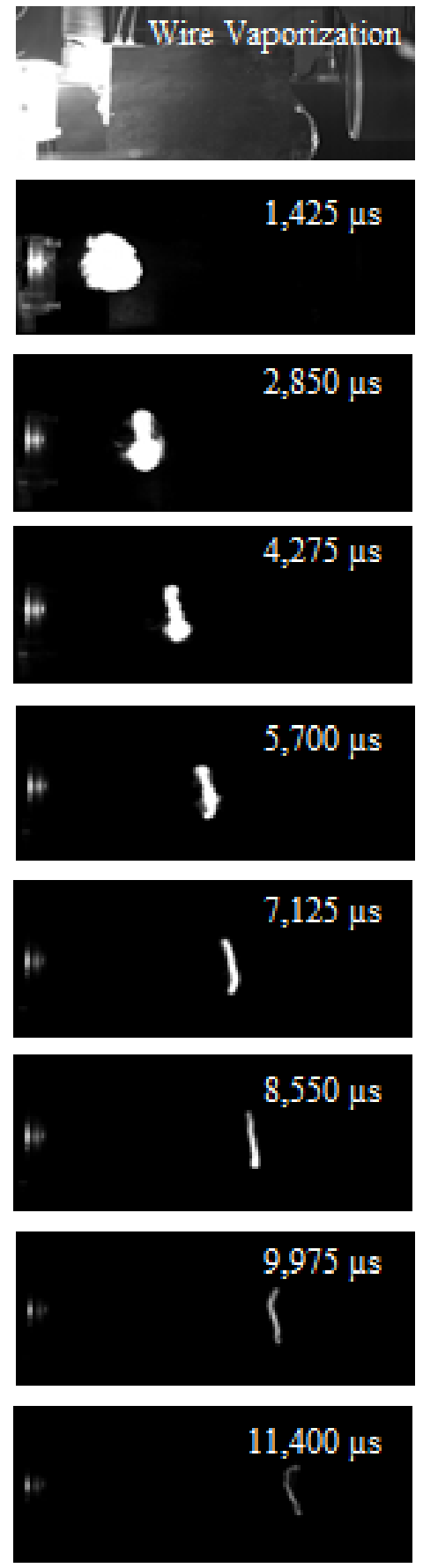

$12,825 \mu \mathrm{s}$
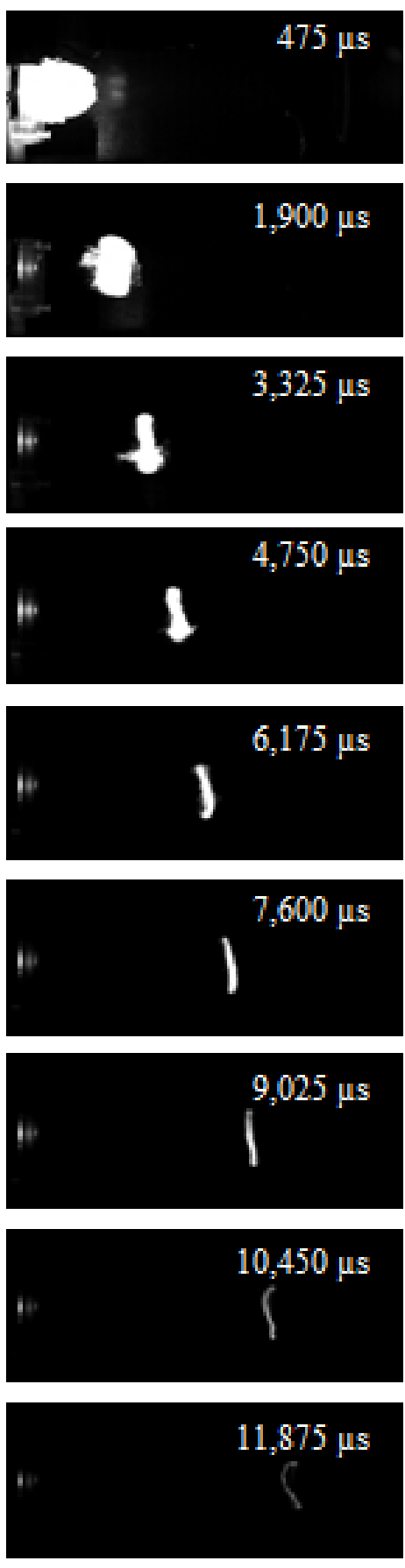

$13,300 \mu \mathrm{s}$
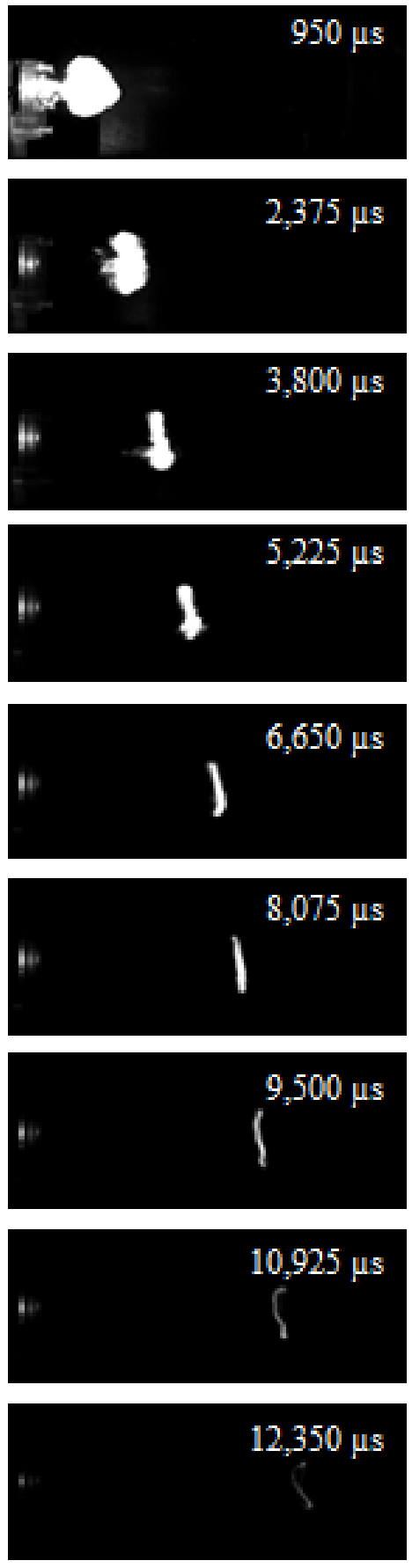

$13,775 \mu \mathrm{s}$
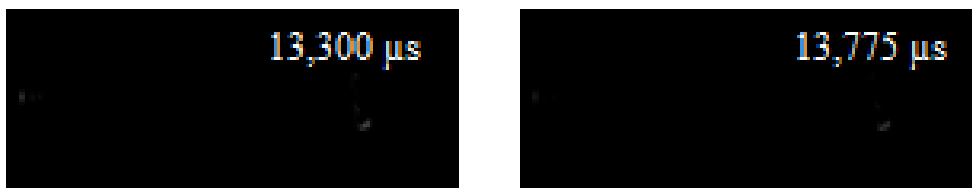

Figure 5.1 High speed images of the TAP. This discharge was produced with the circuit and test fixture conditions described in Section 3.2 and 3.3 (4.4 kJ stored energy, $30 \mathrm{kV}$ charge voltage, $6 \mathrm{kA}$ discharge current, fixture length $0.1 \mathrm{~m})$. The discharge traveled approximately $0.4 \mathrm{~m}$ over $12.4 \mathrm{~ms}$. 
As previously discussed, rather than using the traditional minor and major radii, we typically describe the TAP by its height and width from a side axis perspective. These dimensions are shown in Figure 5.2. These dimensions are used for convenience when the camera is positioned in the side axis perspective. The minor and major radii of the TAP will be discussed later in this chapter.

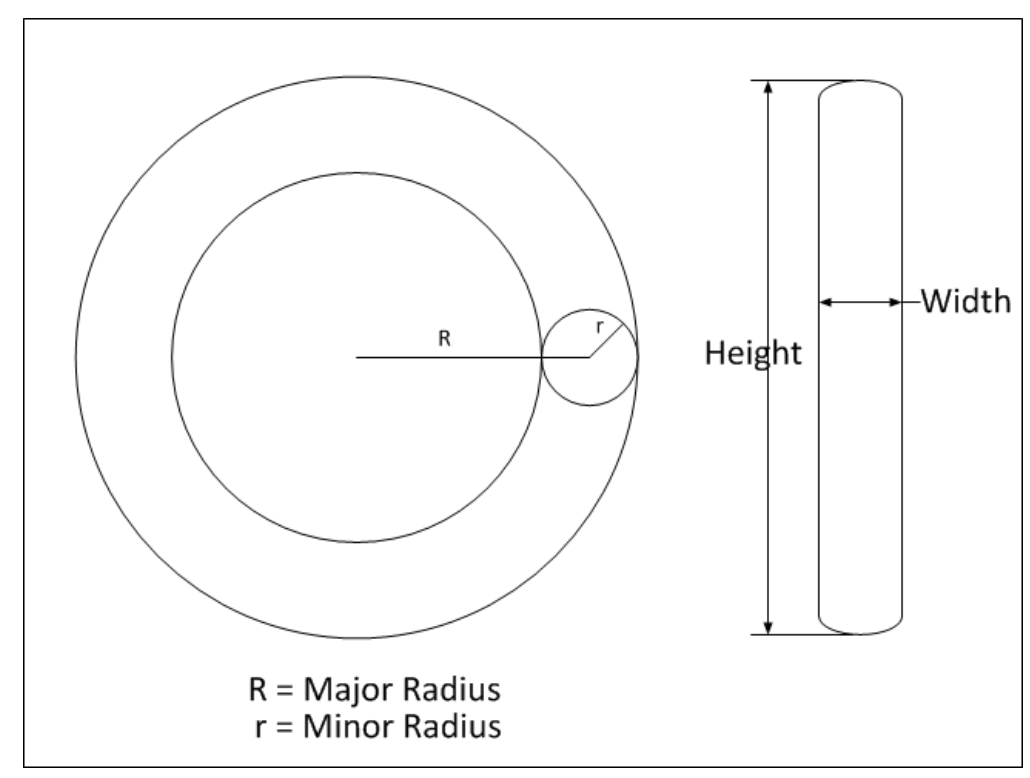

Figure 5.2 Toroid dimensions of the image processing analysis of the TAP

For an enhanced view, Figure 5.3 consists of selected duplicate images of Figure 5.1. Figure 5.3 shows images which are $950 \mu \mathrm{s}$ apart with approximate dimensions of the discharge. Once detached, the average velocity of the plasma is $31.37 \mathrm{~m} / \mathrm{s}$.

In all of these images, the stationary discharge between the two accelerator screens was observed. This discharge is sustained by the secondary electrolytic capacitor bank as previously described. The secondary discharge is present for several tens of milliseconds after the TAP, but the accelerator bank has ceased interacting with the TAP once detachment occurs. 

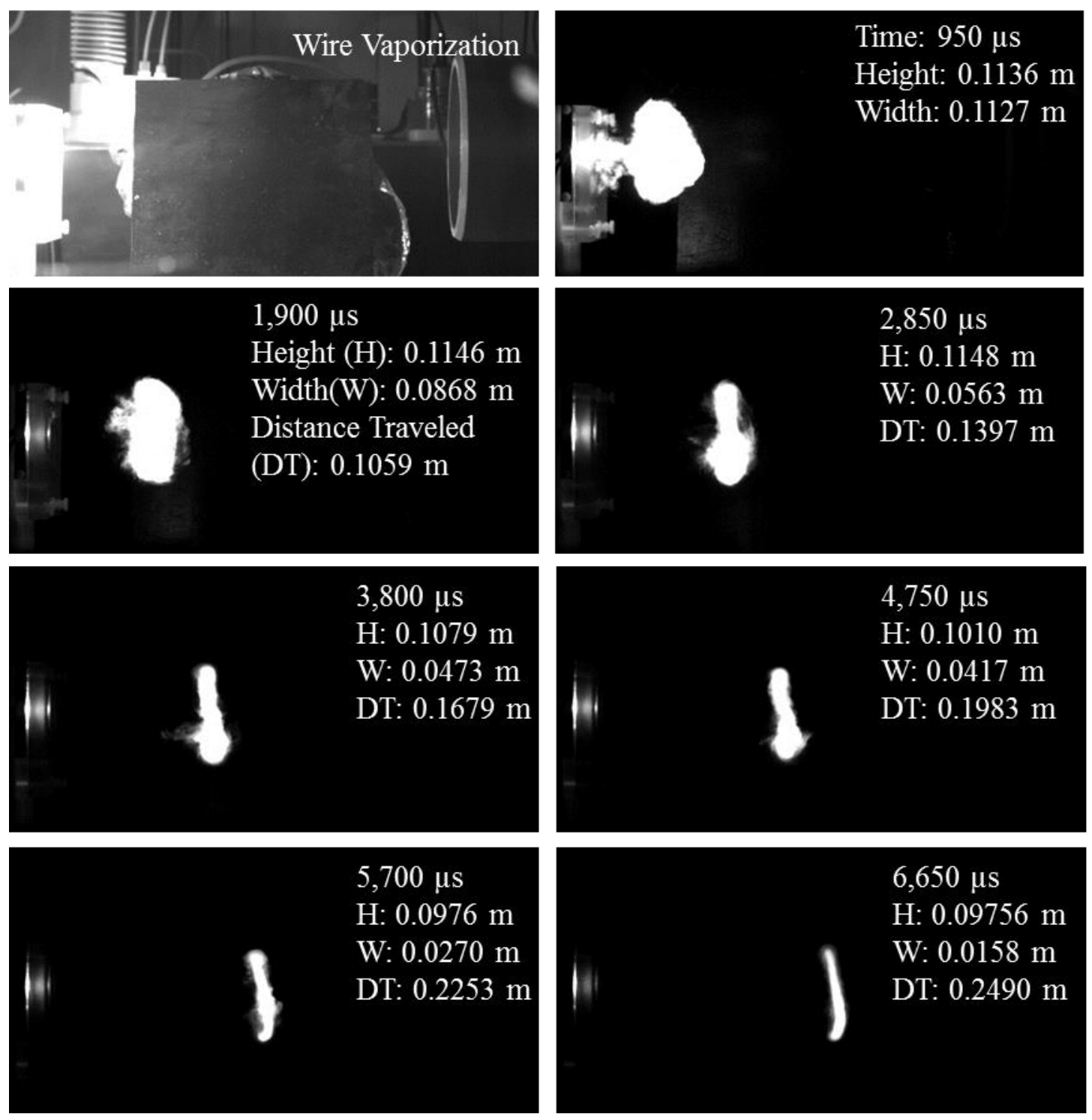

Figure 5.3 Selected images from Figure 5.1 with details on timing and dimensions of the discharge. The height $(\mathrm{H})$, width (W), and distance traveled (DT) are given. This discharge was produced with the circuit and test fixture conditions described in Section 3.2 and 3.3 (4.4 kJ stored energy, $30 \mathrm{kV}$ charge voltage, $6 \mathrm{kA}$ discharge current, fixture length $0.1 \mathrm{~m}$ ).

Figure 5.4 is a set of images from a high speed video where the camera was placed on-axis with the exploding wire of the TAP. This plasma discharge was from a different trial than the preceding experiment shown in Figure 5.1 and Figure 5.3. Again, this video was recorded at $21,052 \mathrm{fps}$ with a $47.50 \mu \mathrm{s}$ period by the Phantom v7.3 at a $2 \mu \mathrm{s}$ exposure. The images shown in Figure 5.4 are 10 frames, or $475 \mu \mathrm{s}$, apart. The first image on the top left is that of 'first light' taken to be the point of wire vaporization. In 
these images, the evolution of the toroidal shape is easily observed in the discharge at approximately $1.4 \mathrm{~ms}$. The major diameter of the toroid is approximately $0.06 \mathrm{~m}$, remaining between $0.05 \mathrm{~m}$ and $0.07 \mathrm{~m}$ throughout its travel. The minor radius of the toroid decreases as time passes and the plasma thins out. The minor radius begins in the fourth frame at $1.82 * 10^{-2} \mathrm{~m}, 1.425 \mathrm{~ms}$ after vaporization, and decreases steadily to $0.6^{*} 10^{-2} \mathrm{~m}$ in the final frame, at $5.225 \mathrm{~ms}$.
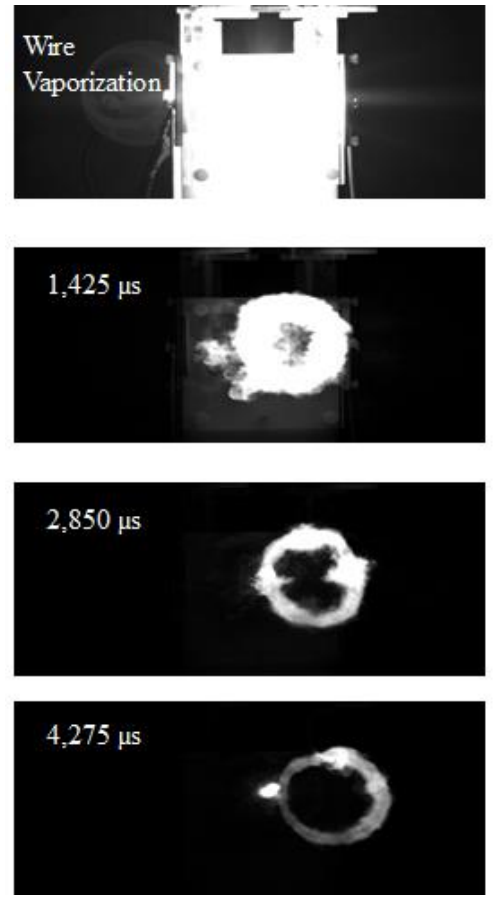
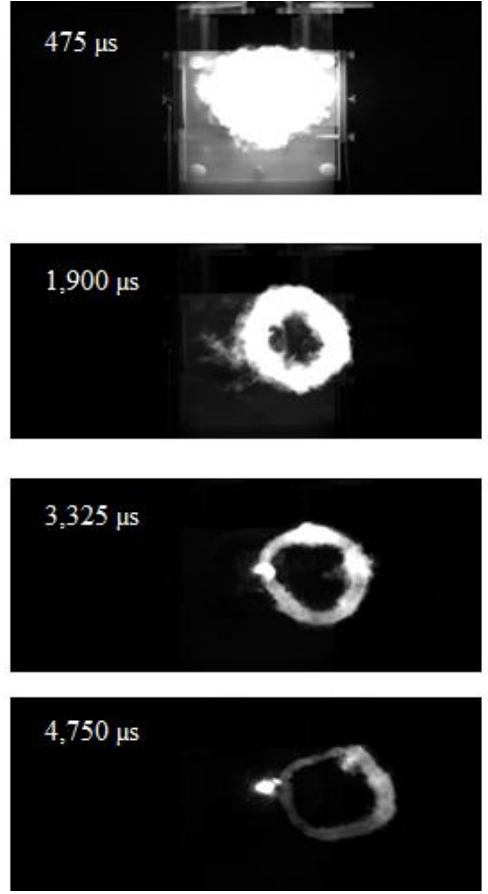
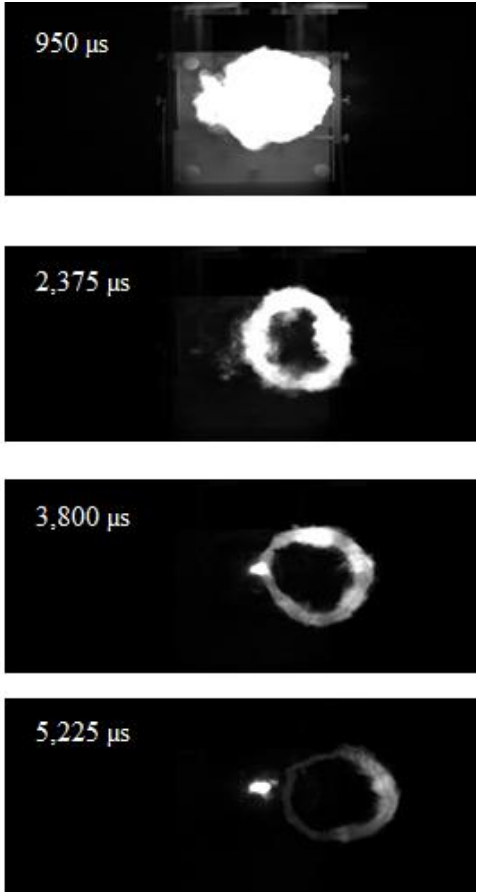

Figure 5.4 High speed on-axis images of the TAP. This discharge was produced with the circuit and test fixture conditions described in Section 3.2 and 3.3 (4.4 kJ stored energy, $30 \mathrm{kV}$ charge voltage, $6 \mathrm{kA}$ discharge current, fixture length $0.1 \mathrm{~m}$ ).

Also of interest is the stationary plasma discharge between the accelerator screens on axis of the plasma fixture. It is important to realize this discharge is always present during each trial, and that in this view, it can be difficult to distinguish between it and the TAP. In the later images of Figure 5.4, it is obvious that the TAP has drifted off axis. This is not uncommon, although the direction of drift varies between trials. 


\subsection{Variability in the TAP}

Despite the complicated nature of the toroidal air plasma discharge, it was reproducible. Variability in longevity, size, direction of travel, transient signals of the current and voltage traces, and other quantitative results was observed between trials. Over two hundred TAP discharges were fired and each was slightly different in these characteristics. These variations are likely due to small variations in surface conditions of the wire, resistance in the pulsed power circuit (the aqueous electrolyte resistor), temperature and humidity in the laboratory, and local instabilities within the TAP. Each experiment was completed in repetition to get a basic understanding of the qualitative results.

The timing at which the TAP fired was of critical importance in the shock wave experiments. Wire vaporization occurred about $2.2 \mathrm{~ms}$ from system trigger, with a jitter within the interframe time of the Phantom camera, which is $47.5 \mu \mathrm{s}$. The expansion, evolution, and formation of the toroid varied, but were still within practical limits for interaction with external events (i.e. shock wave). The size and shape of the plasma is discussed further in the following sections, and it is important to keep in mind that the goal in these experiments was to have a large plasma in a specified region for at least $1 \mathrm{~ms}$.

\subsection{Image Processing Analysis of the TAP}

The image processing algorithm, described in Section 4.3 and Appendix C, will be used throughout this dissertation as a means to compare results of different experiments. The remainder of this section will discuss the results of this image processing analysis on a series of experiments. These successive experiments were performed using the typical 
experimental configuration presented in the previous sections of this chapter (with fixture length of $0.1 \mathrm{~m}$ ). The same data will then be compared to experimental variations on the plasma source in Chapter 6.

\subsubsection{Height}

The height data of four TAP discharges are shown in Figure 5.5. The graph of the height data of these TAP discharges effectively illustrates their variability. The sudden decrease in height of each curve corresponds to the dissipation of the plasma discharge. As shown, the duration of the discharge is variable between experimental trials, ranging from $8,598 \mu$ s to $12,450 \mu \mathrm{s}$ in these trials. Another interesting feature is that the height of each plasma discharge stays relatively constant between $0.124 \mathrm{~m}$ and $0.096 \mathrm{~m}$ for the lifetime of the plasma.

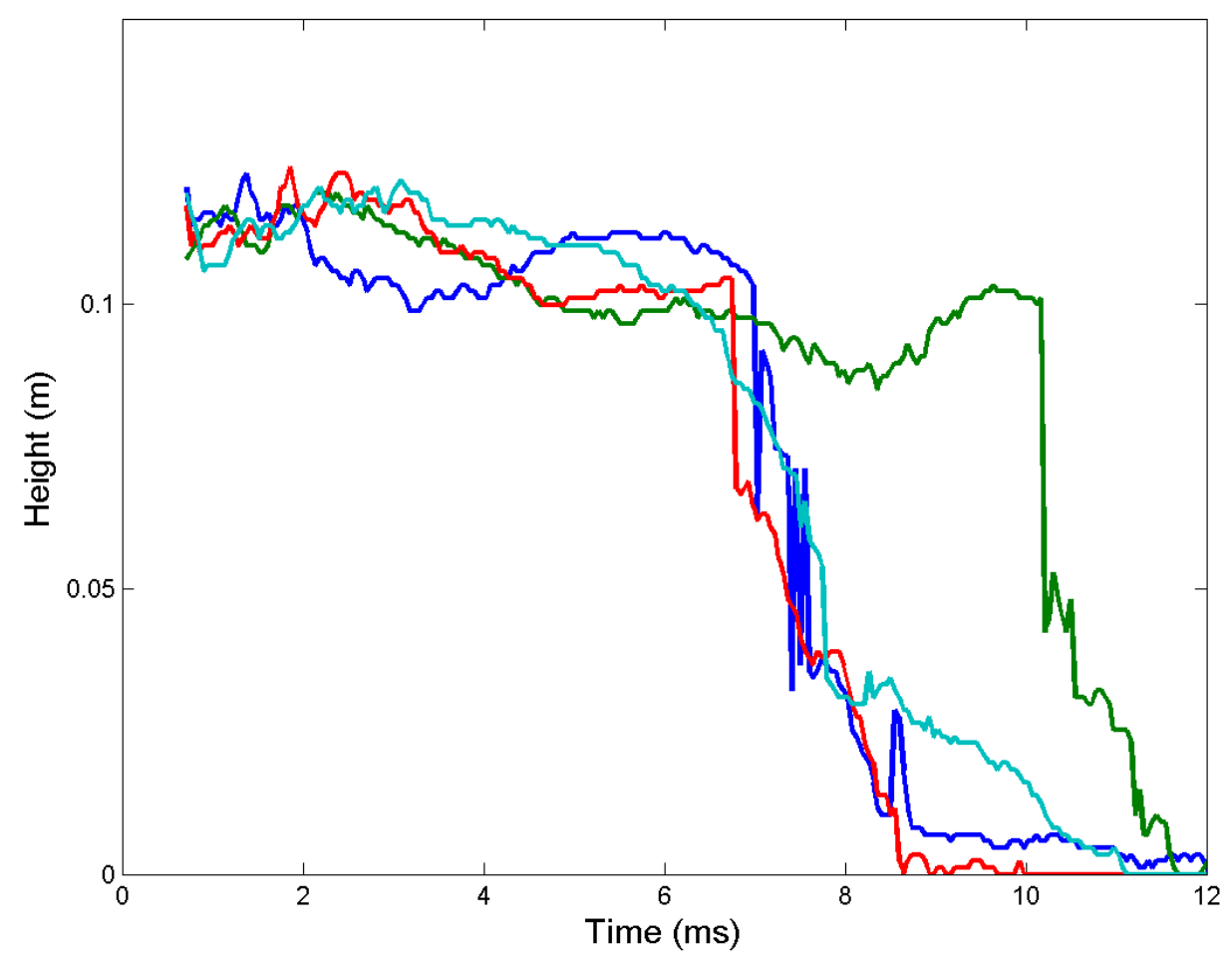

Figure 5.5 Height data of four TAP discharges 


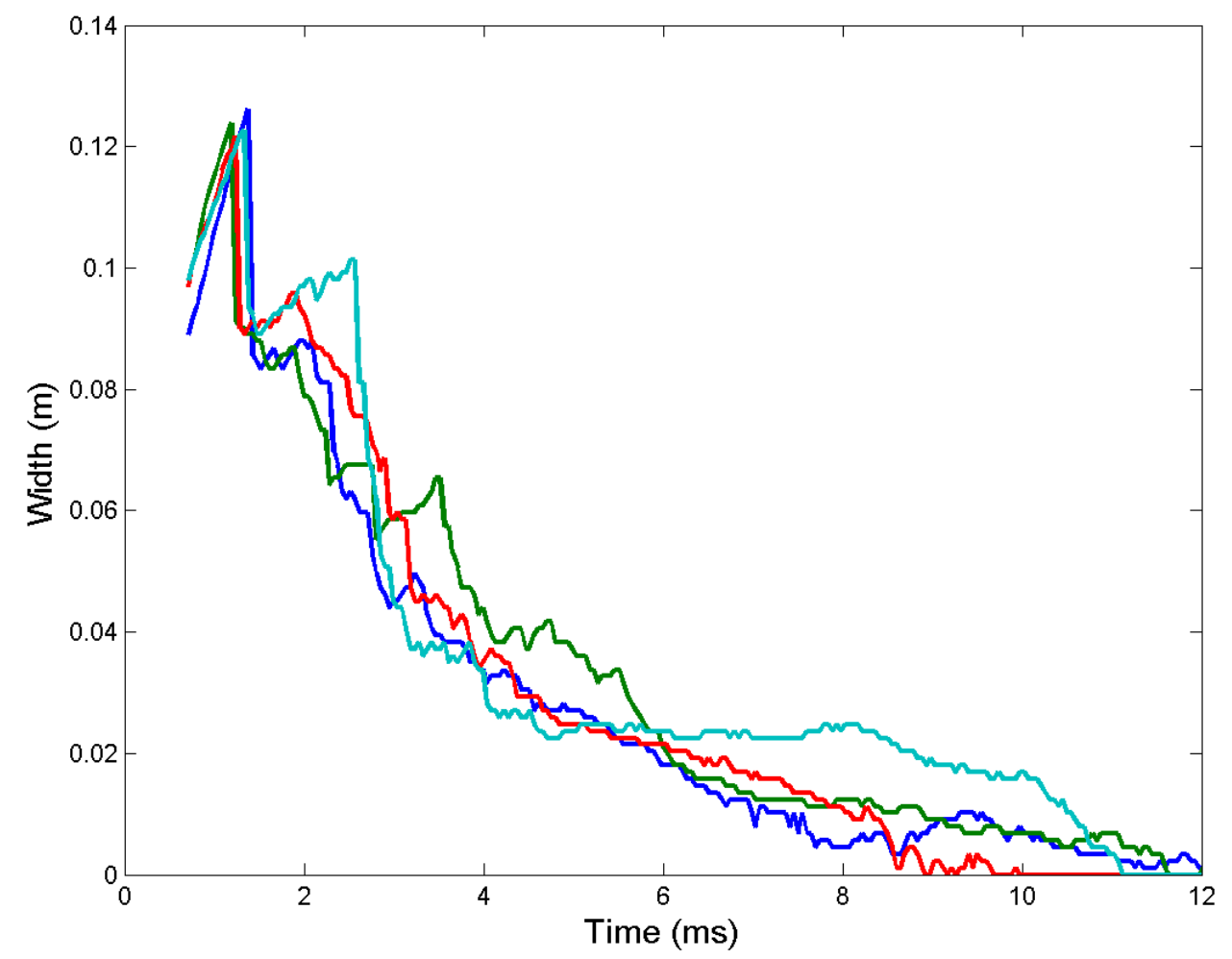

Figure 5.6 Width data of four TAP discharges

\subsubsection{Width}

The width data of four TAP discharges are shown in Figure 5.6. If this figure is reconciled with its corresponding videos, it is apparent that the spike in width around 1250-1350 $\mu$ s coincides when the plasma detaching from the fixture. Data before this peak is omitted as it is significantly influenced by blooming. This graph shows that the width steadily decreases after detachment.

\subsubsection{Cross Sectional Area}

The cross sectional area data of four TAP discharges are shown in Figure 5.7. This is calculated by adding the illuminated pixels in the image processing algorithm, described in Section 4.3 and Appendix C. Beyond the blooming effects of the initial frames of the 
video (data omitted), the plasma discharge decreases steadily in area from about 0.0090 $\mathrm{m}^{2}$ at maximum until it has dissipated.

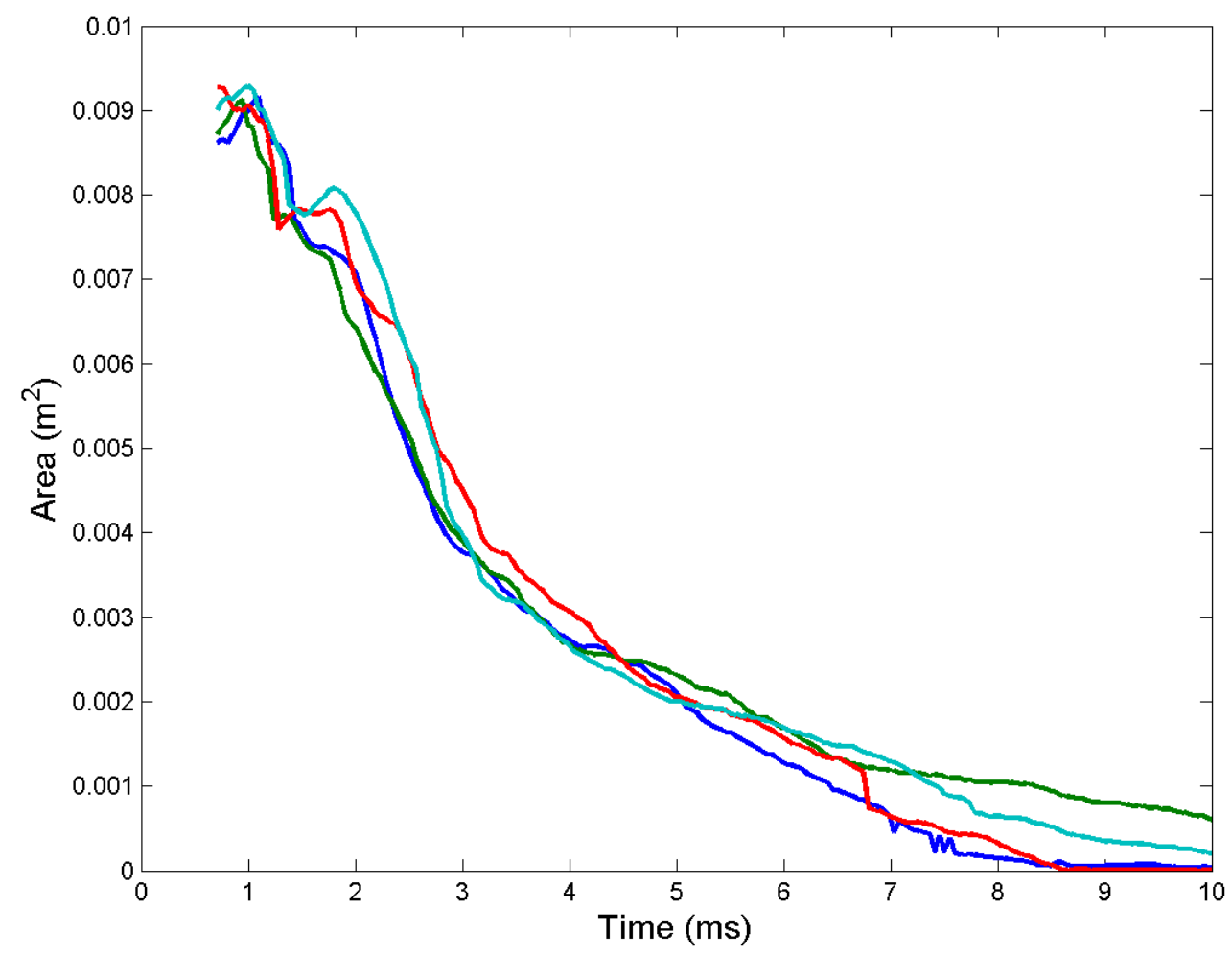

Figure 5.7 Cross sectional area data of four TAP discharges

\subsubsection{Distance Traveled}

The distance which each of the four TAP discharges traveled is shown in Figure 5.8. The plasma travels away from the fixture with a nearly constant velocity, between 20 and $35 \mathrm{~m} / \mathrm{s}$. These curves also confirm the plasma lifetimes seen in the height data. The longest lasting discharge traveled over $0.35 \mathrm{~m}$ from the fixture, and the shortest duration discharge traveled approximately $0.30 \mathrm{~m}$ from the fixture. 


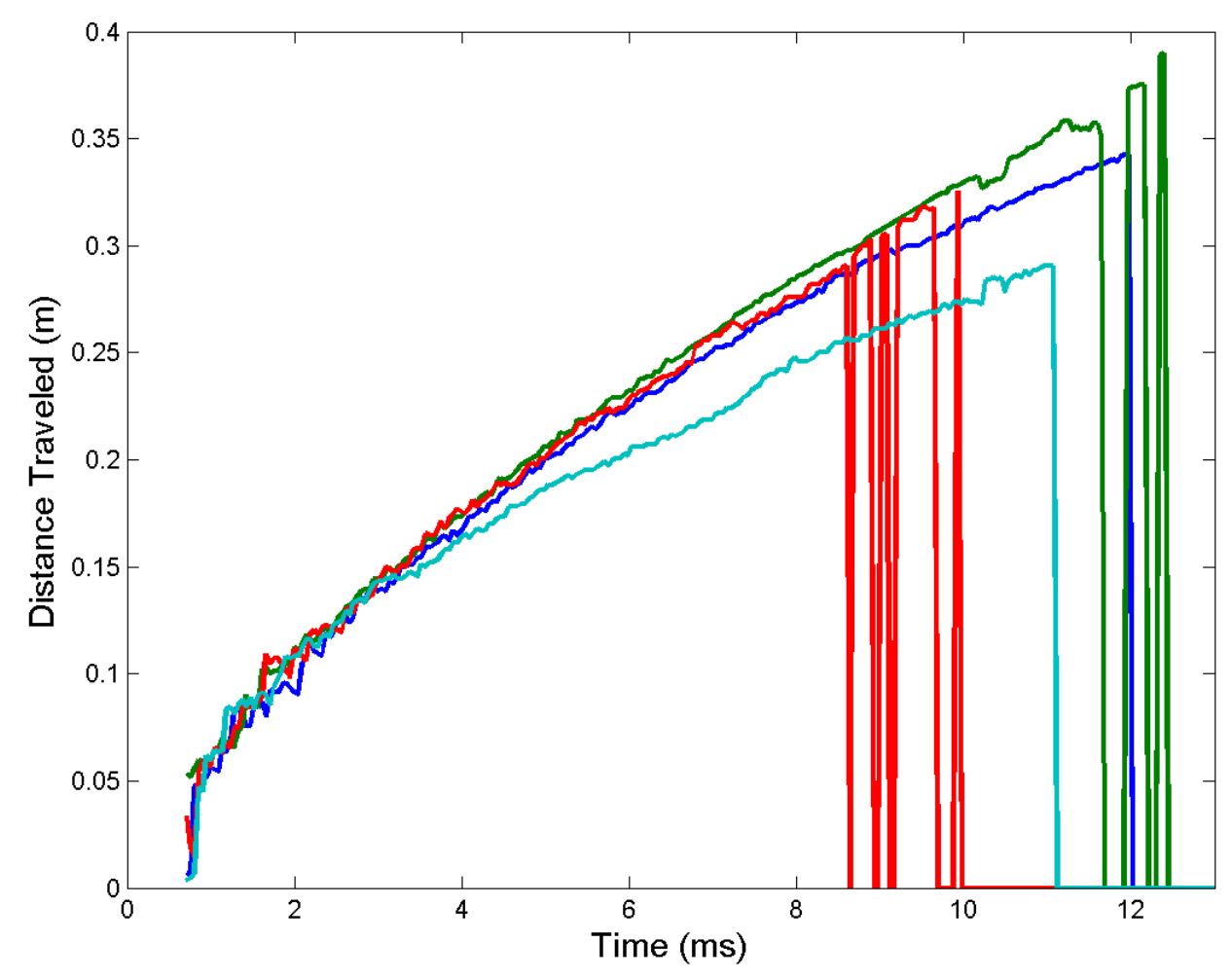

Figure 5.8 Distance traveled data of four TAP discharges

\subsubsection{Major Radius and Minor Radius}

The program was modified to track the major and minor radii of the TAP from a high speed video with an on-axis view. Details on the implementation of this modified version of the image processing algorithm are given in Appendix C. Five experimental trials were conducted with the Phantom 7.3 observing the TAP from an on-axis perspective.

The toroid shape of the TAP is not a perfect circle, but averaging the height and width of the TAP yielded an approximation for the diameter of the toroid. This diameter was then divided by 2 to obtain a radius of the toroid, but this radius is not the major radius of the toroid, as shown in Figure 5.2. This approximate radius was decreased by the size of the minor radius to obtain the major radius of the TAP for each image of the high speed video. These details are further explained in Appendix C. 
The major radii of the TAP discharges are shown in Figure 5.9 along with an average of the data (shown as a thick black line). The TAP major radius was approximately $0.0525 \mathrm{~m}$ at $1 \mathrm{~ms}$, dropped to $0.045 \mathrm{~m}$ at approximately $1.75 \mathrm{~ms}$ until its steady decline starting at approximately $2.75 \mathrm{~ms}$, reaching $0.02 \mathrm{~m}$ near $5 \mathrm{~ms}$ on average. Figure 5.9 shows several trials increase in major radius after an initial decrease, this is observed in the high speed videos as an increase in width of the TAP as it has fully expanded out of the test fixture (note that this is not the same width as in Section 5.3.2, because the high speed camera is in an on-axis view in this case).

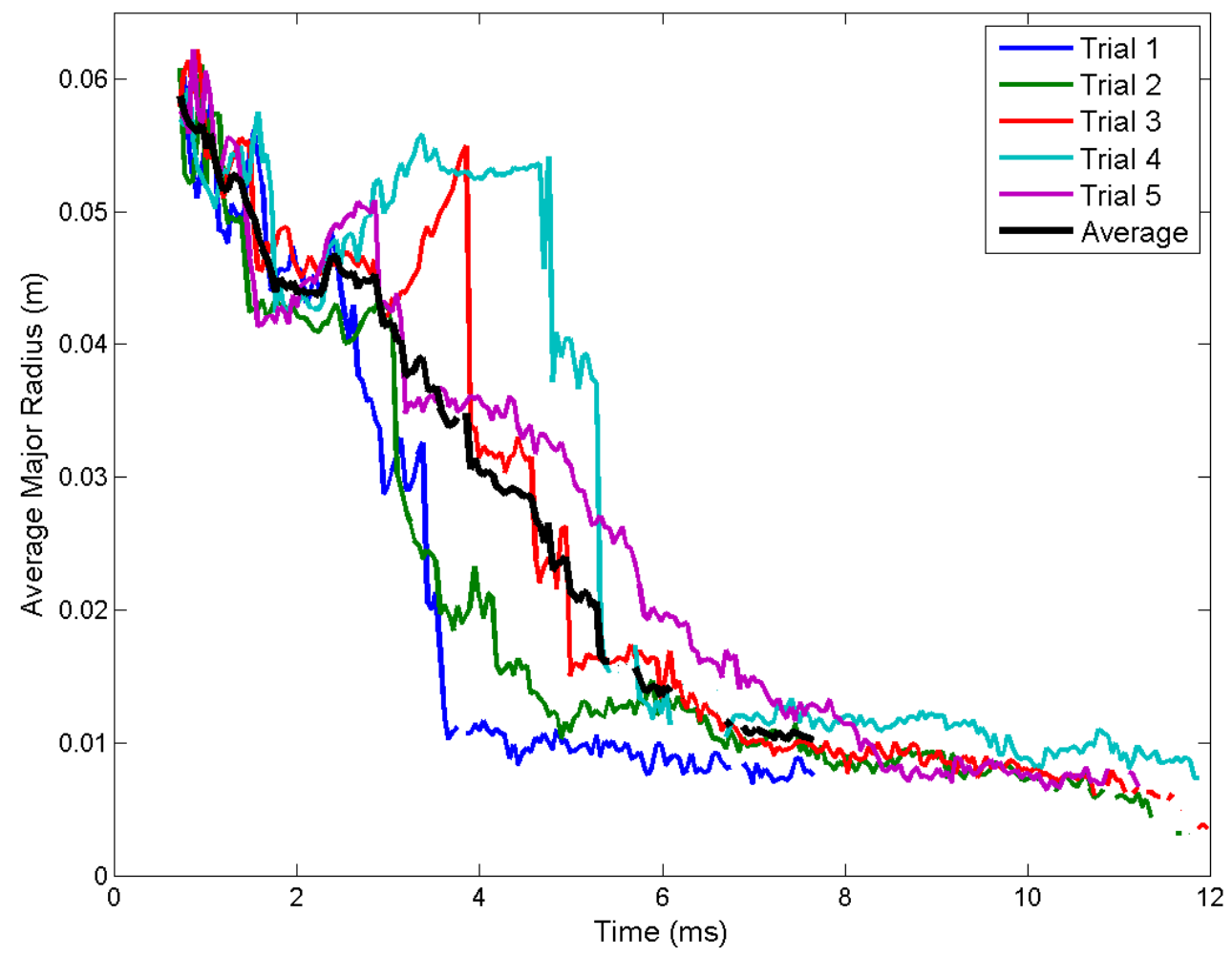

Figure 5.9 Major radius data of the TAP

The minor radii are shown in Figure 5.10 along with an average of the data (shown as a thick black line). The TAP minor radius was approximately $0.025 \mathrm{~m}$ at $1 \mathrm{~ms}$ and dropped to less than $0.005 \mathrm{~m}$ near $6 \mathrm{~ms}$ on average. The inflection in the minor radius of the TAP near $1.5 \mathrm{~ms}$ is associated with the detachment and formation of the toroid shape. 


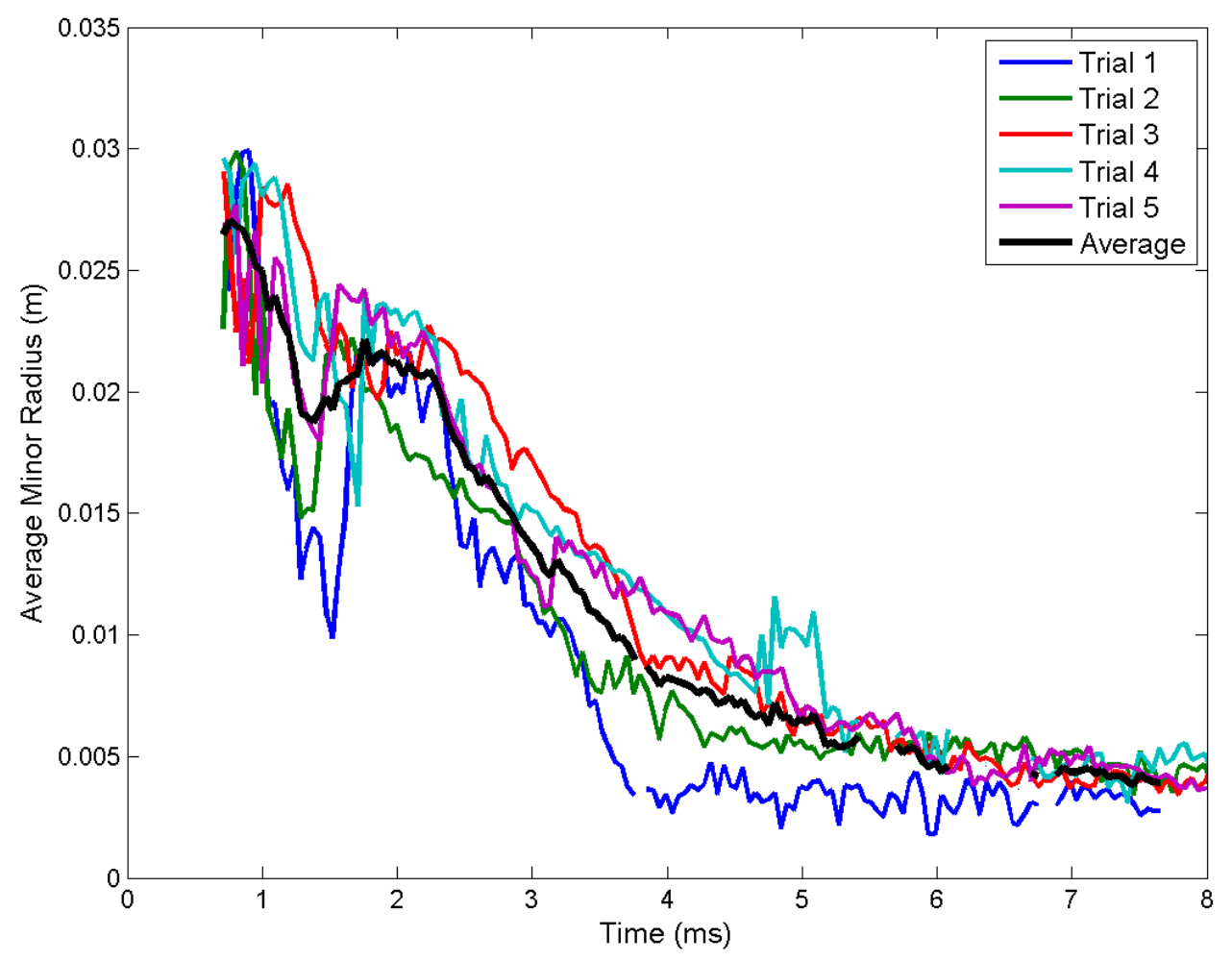

Figure 5.10 Minor radius data of the TAP

\subsection{Interaction Considerations}

When reviewing the background theory of interaction completed by previous researchers presented in Chapter 1, it is apparent that the plasma should be as large in volume as possible when interacting with the shock wave. While the TAP is less dense in its center than at its edges, and thus not ideal for mitigation of a shock wave, it is the best plasma source yet in terms of size and duration.

The experiments were designed to interact the shock wave with the TAP after it detaches from the stationary fixture. In practice, this interaction occurred approximately $2,500 \mu \mathrm{s}$ from plasma vaporization. Thus, it was important to characterize the plasma in this stage of its evolution. As the preceding sections have presented, this is when the plasma is at its largest height and width. At 2,500 $\mu$ s the plasma is $0.11-0.12 \mathrm{~m}$ in height, 
and $0.08-0.10 \mathrm{~m}$ in width from the side axis perspective of the Phantom camera. From the on-axis experiments, the TAP is approximately a torus with a $4.5-6^{*} 10^{-2} \mathrm{~m}$ major radius when the shock wave arrives.

\subsection{Accelerator Region Investigation}

Early experiments with the TAP, discussed in Section 2.2.4, determined that the accelerator region had a significant effect on the formation of the toroidal shape and the duration of the plasma. These experiments were duplicated with the addition of the Phantom 7.3 high speed camera and the image processing algorithm. A series of experiments were conducted to characterize the effects of the accelerator region on the plasma size and duration. Four accelerator conditions were tested and compared with five discharges per condition. The four configurations were:

1. Without accelerator grid

2. With accelerator grid, $\mathrm{V}=0$

3. With accelerator grid, $\mathrm{V}=100$

4. With accelerator grid, $\mathrm{V}=250 \mathrm{~V}$

The results of these experiments are shown in Figure 5.11 and Figure 5.12. The area data is shown in Figure 5.11, and the distance traveled data is shown in Figure 5.12. 
The largest effect is observed when comparing the 'without Screen' condition (shown in blue) with the other conditions with the screen. For four of the five trials, removing the accelerator grid resulted in a plasma discharge which did not form a toroid and had significantly reduced duration as observed by the high speed cameras. Charging the accelerator circuit and discharging energy into the plasma increases the area of the plasma discharge, at least in the first $3 \mathrm{~ms}$ (Figure 5.11), but does not have as significant of an effect on the duration of the TAP (Figure 5.12). To maximize the size of the TAP, the accelerator circuit was charged fully to $250 \mathrm{~V}$ for all other experiments. The optimization of the grid separation and voltage was not explored fully during the course of the experiments and needs to be investigated in future work.

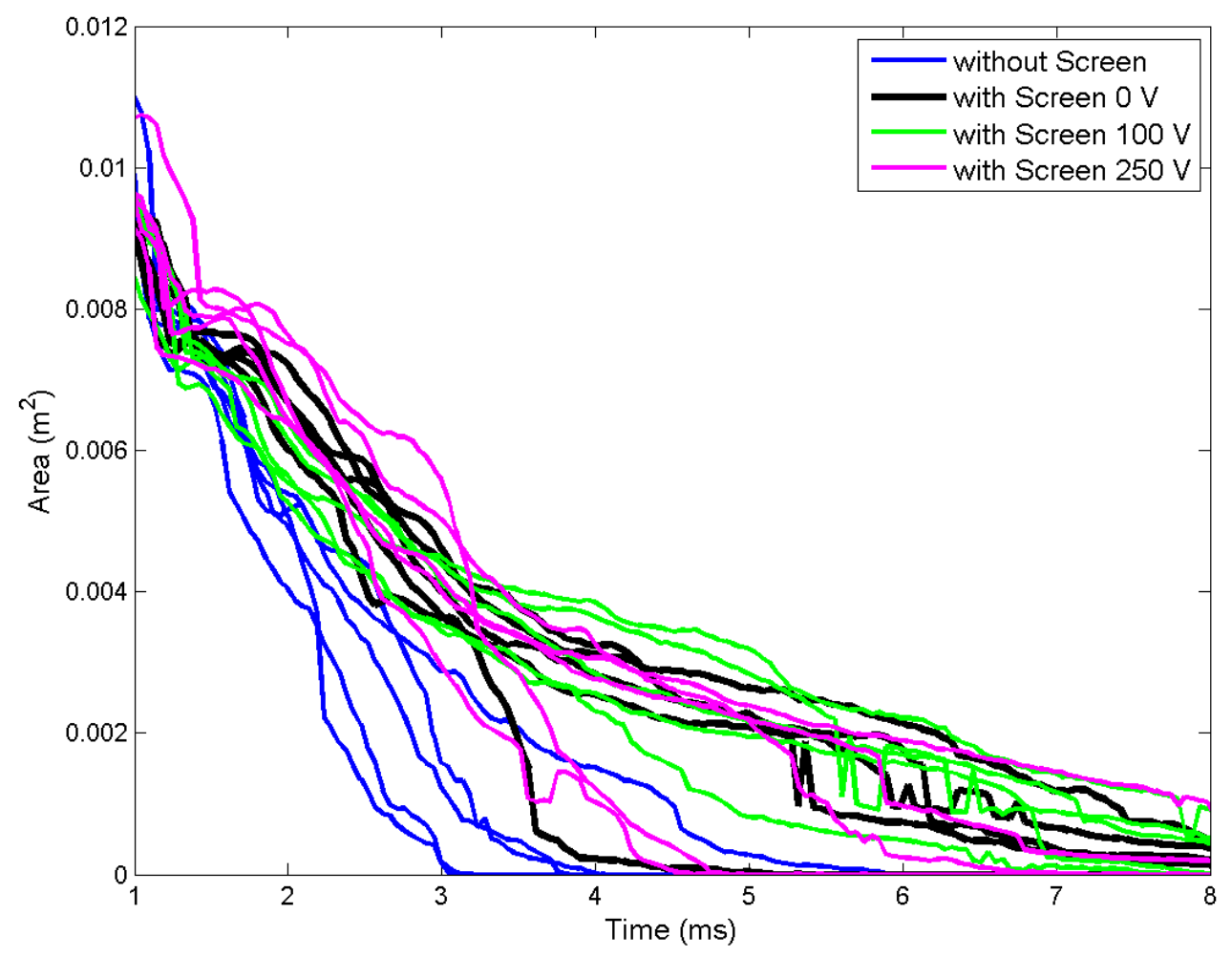

Figure 5.11 Area data for the accelerator region investigation experiments 


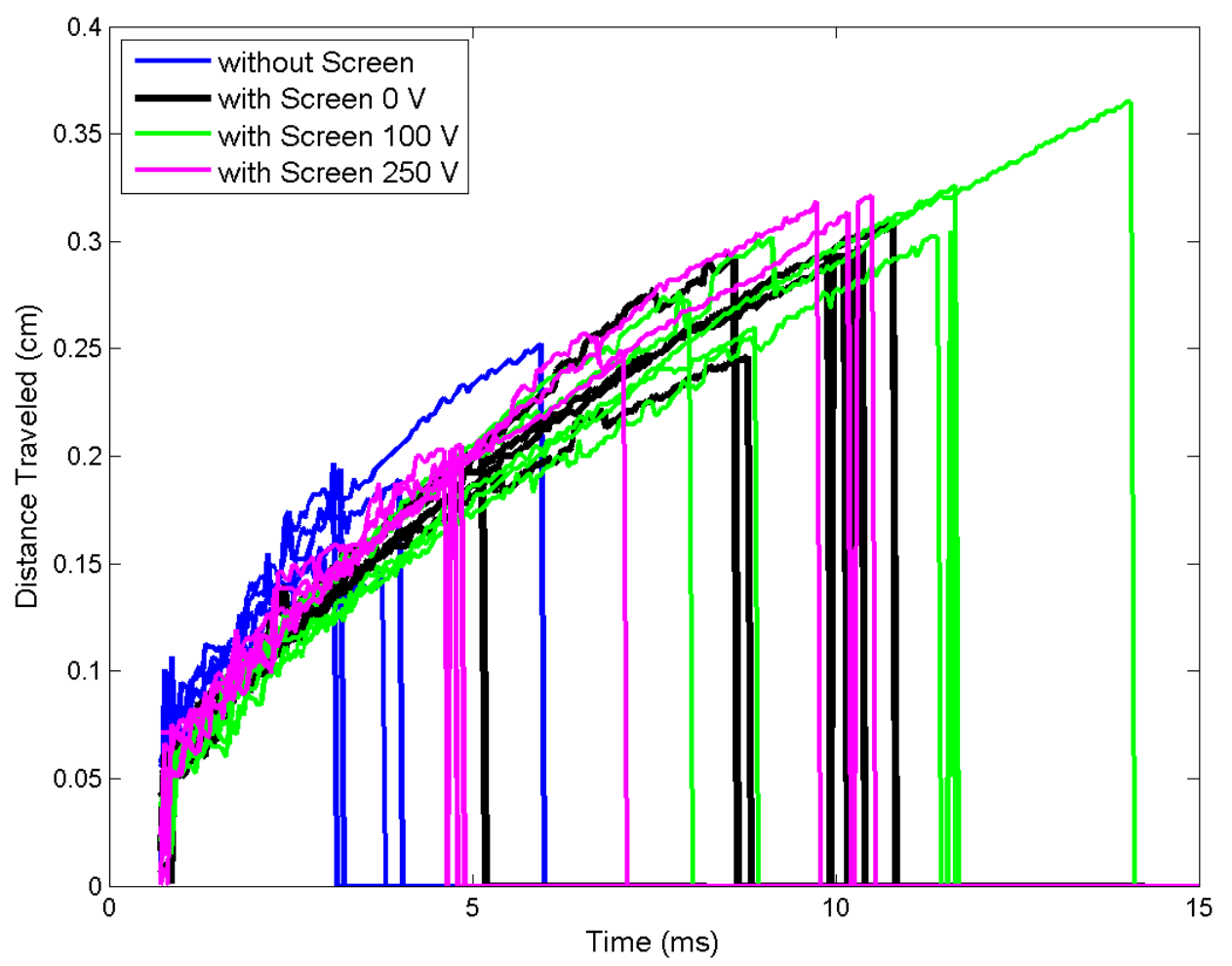

Figure 5.12 Distance traveled data of accelerator region investigation experiments

\subsection{Calculations from the Observed Plasma Current and Voltage Waveforms}

\subsubsection{Comparison of Simulated and Observed Waveforms of the Plasma Circuit}

As discussed in Section 3.1 and Appendix A, a MATLAB ${ }^{\circledR}$ program has been developed at UMC to model the current, voltage, and resistivity of an exploding wire load over time [1]. The model was used to simulate the exploding wire which is used to inject plasma into the accelerator region, and the results were presented in Section 3.1. These simulated results can be directly compared to the observed current and voltage traces presented in Chapter 4.

In an effort to match the simulation parameters to observed data, the modeled circuit resistance was lowered to $4.4 \Omega$ instead of $5.2 \Omega$, and the stray inductance in the circuit was increased to $12.7 \mu \mathrm{H}$ from $12 \mu \mathrm{H}$. As shown in Figure 5.13 and Figure 5.14, these 
parameters more accurately model the physical circuit. From this analysis we can conclude the circuit resistance is likely less than that measured, and the inductance of the circuit is likely higher than measured, expectedly due to the expansion of the plasma discharge. This adjusted simulation calculated an additional $50 \mathrm{~J}$ of energy deposited into the wire, peaking at approximately $100 \mathrm{~J}$.

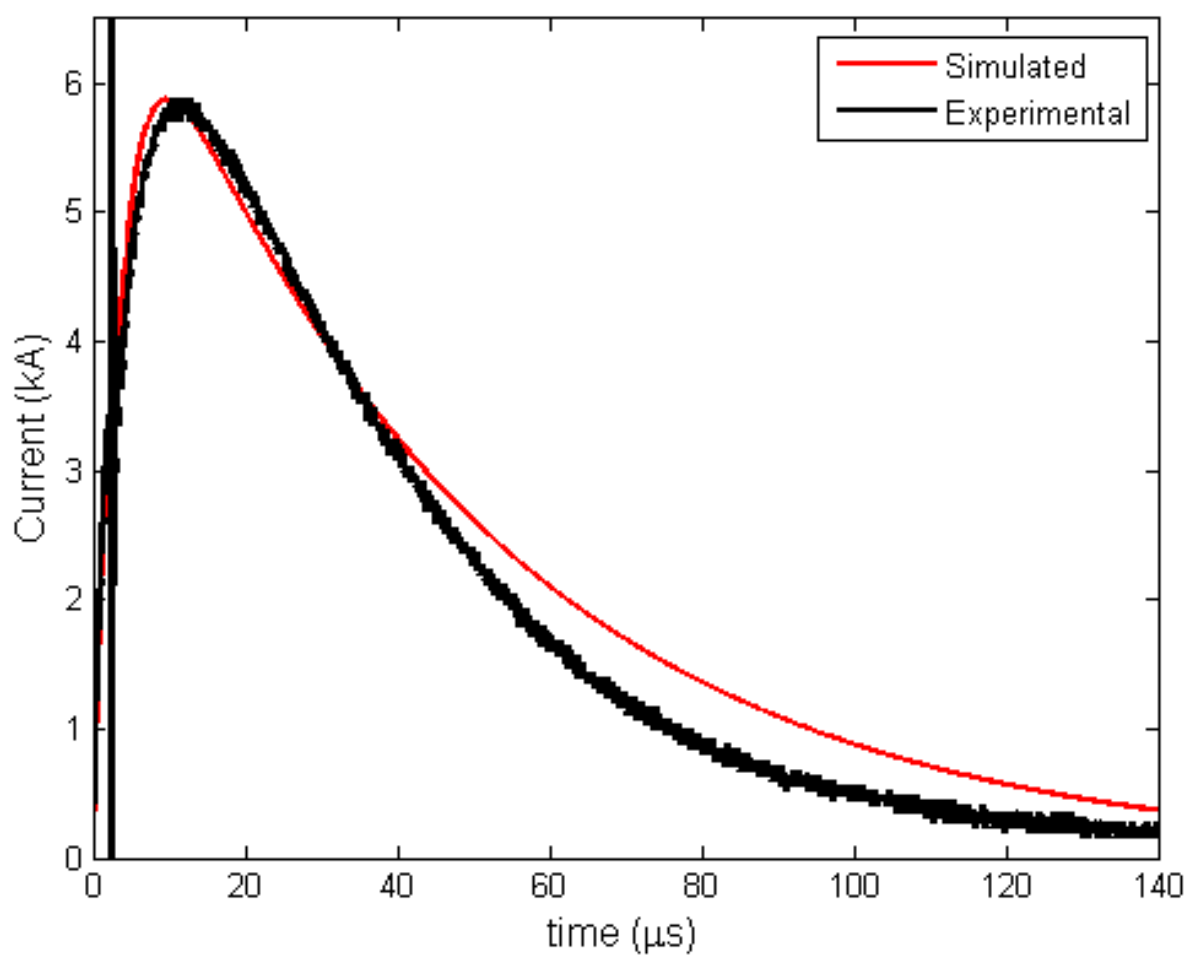

Figure 5.13 Comparison of matched simulation and observed current traces of the exploding wire in the plasma generation circuit 


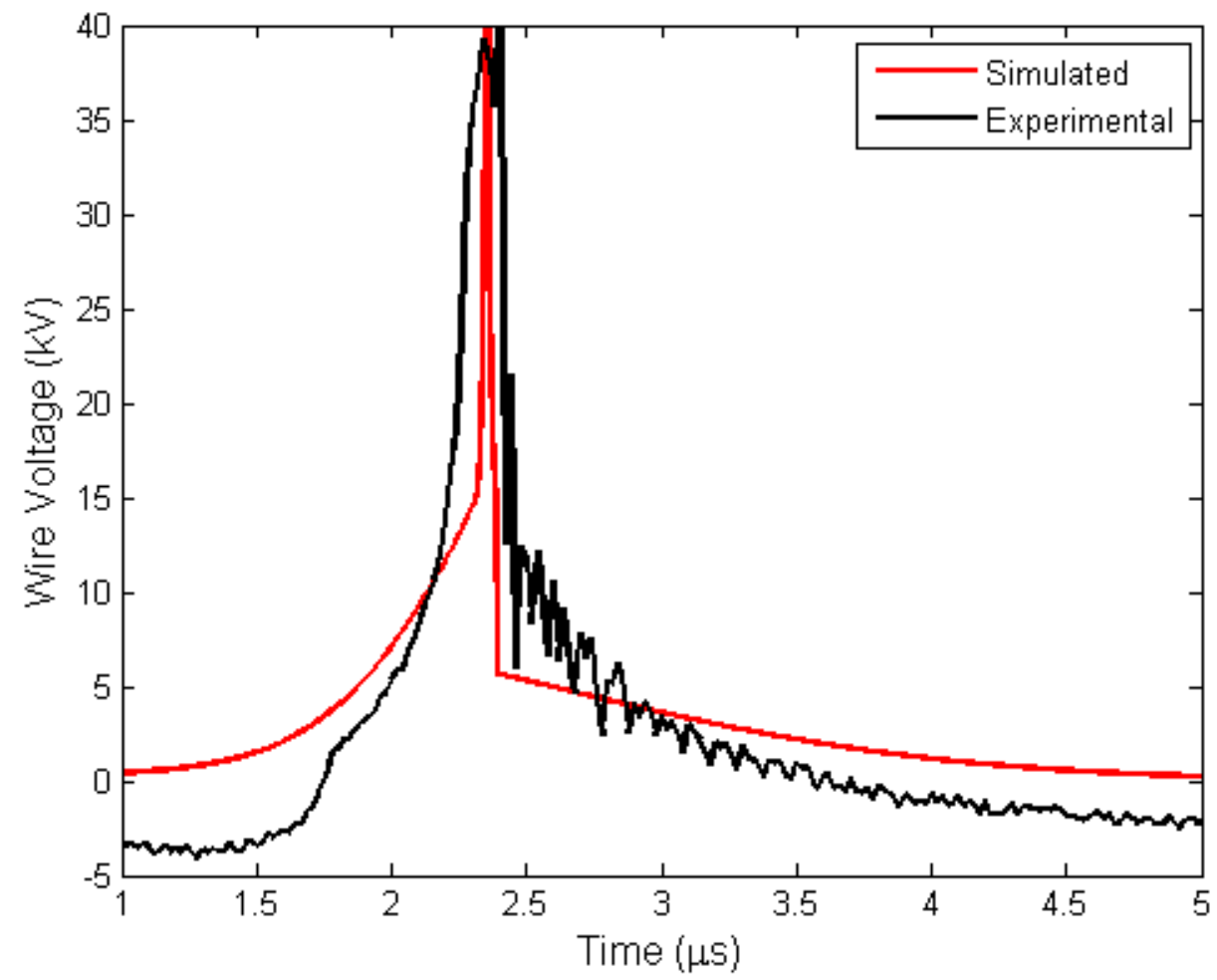

Figure 5.14 Comparison of matched simulation and observed voltage traces of the exploding wire in the plasma generation circuit

\subsubsection{Energy Calculations}

Integrating the measured voltage and current of the exploding wire results in a calculated energy deposited into the wire of approximately $660 \mathrm{~J}$ into the wire over the first $150 \mu$ s, as shown in Figure 5.15. This is over ten times the $50 \mathrm{~J}$ energy deposited that is predicted by simulation. The difference can be explained by the small variations in the observed voltage waveform when compared to the simulated voltage. The measured voltage has periods of negative polarity which the simulation does not predict. These differences are attributed to the changing inductance of the exploding wire as it expands. Attempts at including this changing inductance into the model have created a better experimental match. This dynamic inductance is very difficult to accurately model because the surrounding media is air at atmospheric pressure, and the expansion of the discharge is confined by the test fixture. The simulation is accurate in predicting the 
vaporization of the wire and re-strike but was not designed to accurately predict long duration effects in these conditions. The simulation could model air as a damping medium, but the confining tube geometry causes a complex magnetohydromagnetic effect that would require additional resources to accurately model the experimental results. These effects are not only difficult to model but also difficult to accurately measure, meaning that the actual energy deposited into the discharge from the pulsed power circuit is likely between the $50 \mathrm{~J}$ predicted by simulation and the $660 \mathrm{~J}$ calculated by experimental measurements. The calculated energy deposited shown in Figure 5.15, shows an inflection at approximately $75 \mathrm{~J}$, which corresponds in time with the initial effects that were modeled.

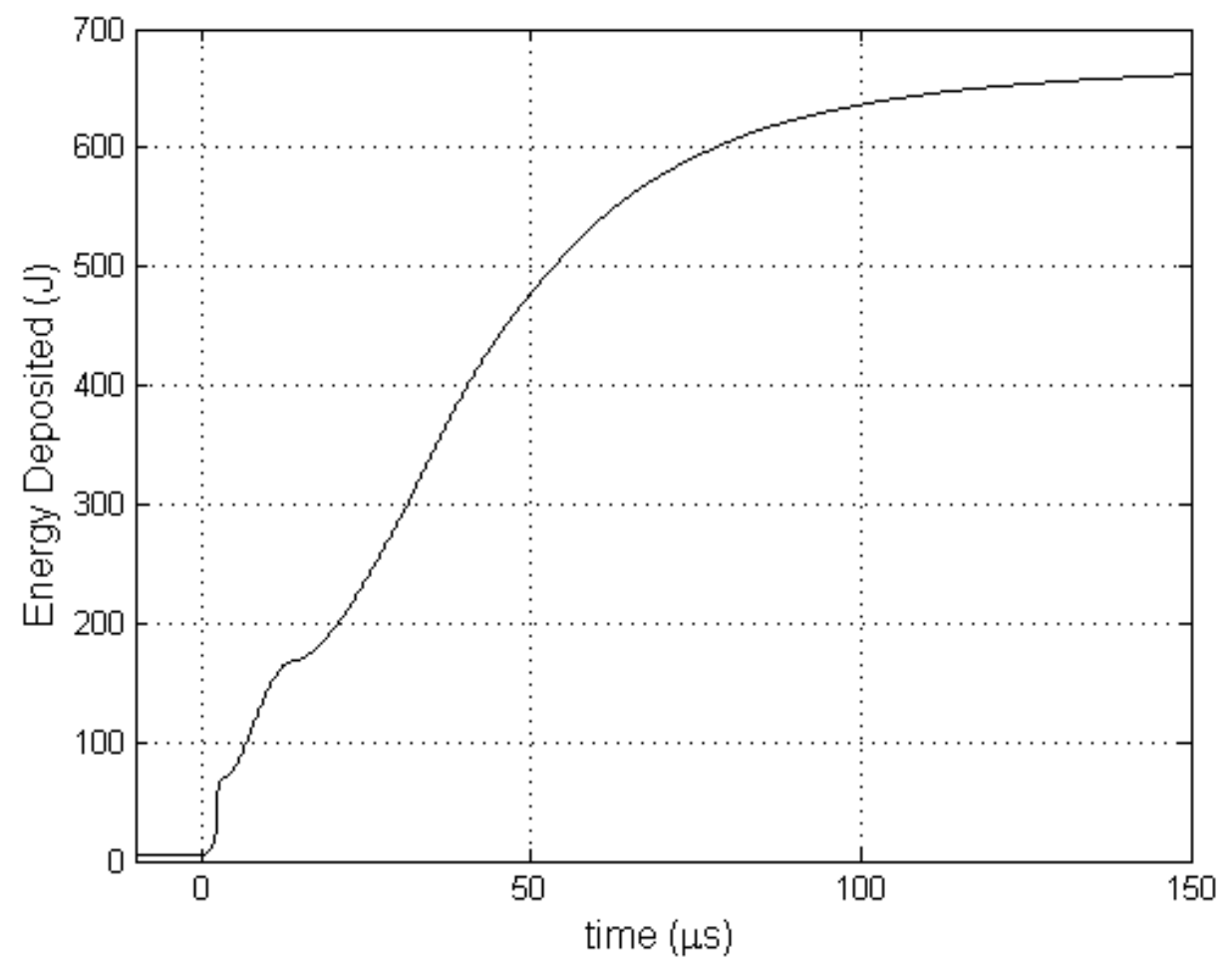

Figure 5.15 Energy deposited into exploding wire as calculated from voltage and current measurements 
The voltage and current monitors on the accelerator circuit were used to calculate an energy deposited into the discharge from the accelerator circuit. Integrating typical current and voltage waveforms of the accelerator circuit, such as those given in Section 4.1, yielded the energy deposited trace shown in Figure 5.16.

As shown in Section 5.1, after an initial period of approximately $18 \mu \mathrm{s}$, the current and voltage waveforms of the accelerator circuit are approximately constant, resulting in a linear energy deposited trace. This condition continues for several milliseconds in duration, fueling the secondary discharge which sustains the current through the accelerator circuit. The data from the first millisecond should accurately reflect the energy deposited into the primary discharge, as the TAP detaches at approximately $1 \mathrm{~ms}$ as shown previously in this chapter. As shown in Figure 5.16, the energy deposited into the discharge from the accelerator circuit over the first millisecond is approximately $32 \mathrm{~J}$.

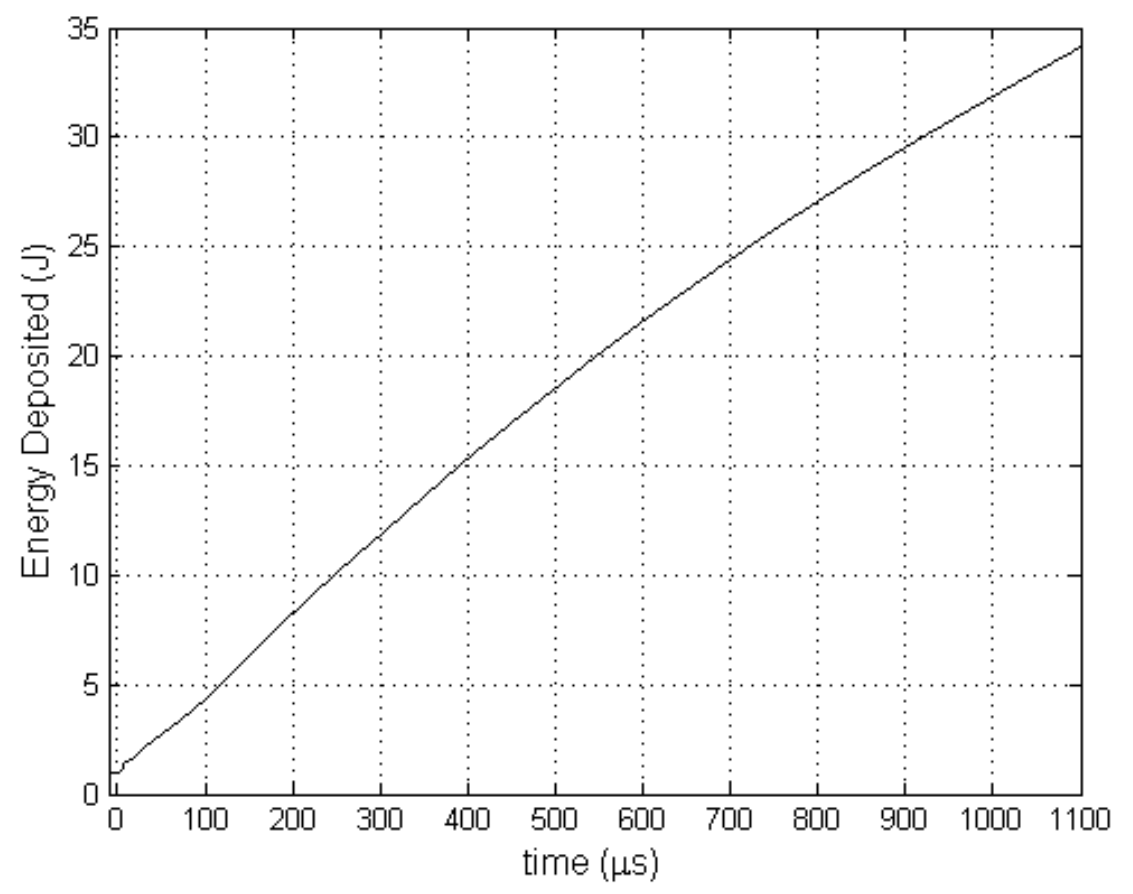

Figure 5.16 Energy deposited into the plasma discharge from the accelerator circuit as calculated from voltage and current measurements 


\subsubsection{Electron Density Calculations}

The electron density $n_{\mathrm{e}}$ can be estimated by measuring the current through an area of the discharge. This can be done for both the current through the exploding wire, and the current between the accelerator screens using Equation 5.1, where $J$ is the current density of the plasma in the area of interest, and $q_{\mathrm{e}}$ is the charge of an electron. The electron drift velocity $v_{\mathrm{d}}$ is estimated from the electric field present in the area of interest [2].

$$
J=n_{e} v_{d} q_{e}
$$

Using the area of the containing tube for the exploding wire and the maximum current that passed through that area $(6.16 \mathrm{kA})$, the peak electron density of the plasma was calculated to be $1.01 * 10^{16} \mathrm{~cm}^{-3}\left(1.01 * 10^{22} \mathrm{~m}^{-3}\right)$ when the wire ignited.

Using the area between the accelerator screens and the maximum current of $432 \mathrm{~A}$, the peak electron density of the plasma in the accelerator region is $1.1 * 10^{14} \mathrm{~cm}^{-3}$ $\left(1.1 * 10^{20} \mathrm{~m}^{-3}\right)$. If accounting for only the metallic cross sectional area of the accelerator screens, then the electron density increases to $2.29 * 10^{14} \mathrm{~cm}^{-3}\left(2.29 * 10^{20} \mathrm{~m}^{-3}\right)$. The plasma between the two screens stays within an order of magnitude of this peak for more than a millisecond, despite the TAP detaching from the region.

\section{References for Chapter 5}

1. K. A. O'Connor, "Compact Power Conditioning and RF Systems for a High Power RF Source," Ms. Thesis, Elec. and Comp. Engr., University of Missouri, Columbia, MO, 2008.

2. L.G. Chrisophourou and D. W. Bouldin, Gaseous Dielectrics V, New York, New York: Pergamon, 1987. 


\section{CHAPTER 6: EXPERIMENTS WITH THE TAP}

After characterizing the TAP, experiments with alterations to the plasma source were conducted to better understand the discharge and to increase the size and duration. Given the novelty of this plasma source and its numerous potential applications, considerable research efforts were focused on scalability.

\subsection{Scaling Experiments}

A series of experiments were conducted to investigate the scalability of the discharge with regard to length of the fixture. The original fixture was $0.10 \mathrm{~m}(4 \mathrm{in})$ in length. A fixture was fabricated which was half this length at $0.05 \mathrm{~m}$ (2 in) and another at twice the original length at $0.20 \mathrm{~m}$ ( 8 in). Figure 6.1 shows the fixtures side by side. The two alternative length fixtures were designed to fit directly into the base in the same manner as the prototypical configuration. All electrical connections, circuit parameters, and diagnostics were operated under the same conditions for each experiment. Changing the length of the fixture also required a corresponding change in the length of the wire.

The results from these experiments were very conclusive; the TAP discharge produced increased in size as the length of the fixture increased. Select images from these experiments are shown in Figure 6.2 as an attempt to visually compare the results in a table of images. 


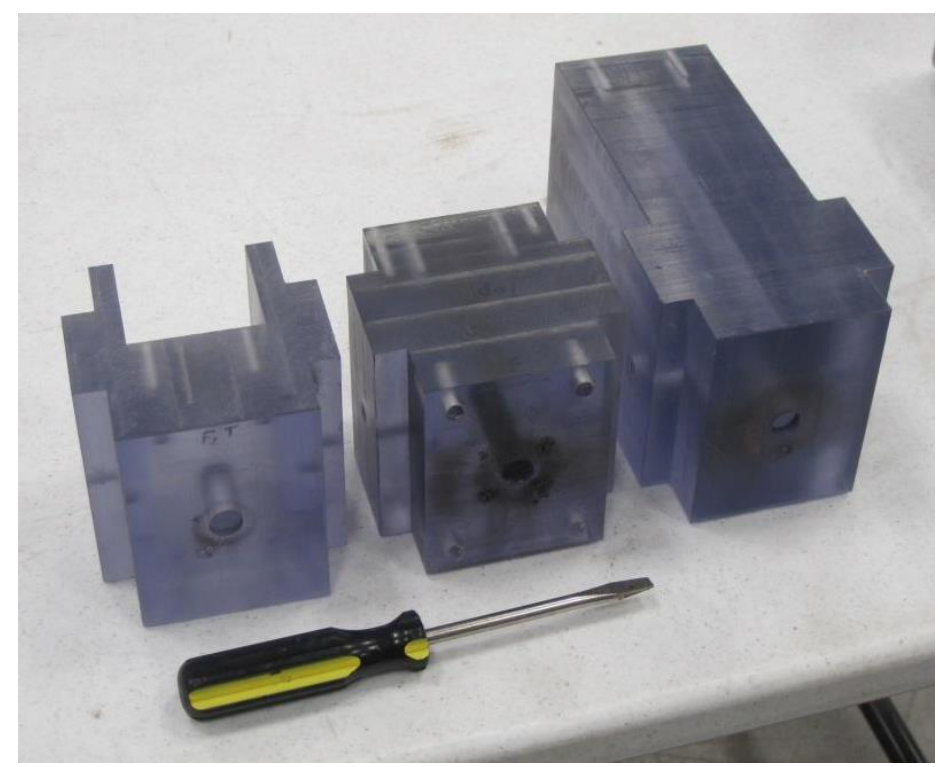

Figure 6.1 Alternative length plasma fixtures

The videos from these trials are even more convincing and were subjected to the same image analysis in MATLAB $®$. The following graphs compare the height, width, area, and distance traveled of the experiments. Figure 6.3 compares the heights of the discharges from these experiments, Figure 6.4 compares the widths, Figure 6.5 compares the areas, and Figure 6.6 compares the distances traveled. From these graphs, it is easy to conclude that the plasmas produced by the $0.20 \mathrm{~m}$ fixture are larger in height, width, and area, than those of the $0.10 \mathrm{~m}$ fixture. Likewise, the plasma discharges from the $0.10 \mathrm{~m}$ fixture are larger than those from the $0.05 \mathrm{~m}$ fixture. The plasma discharges of the $0.05 \mathrm{~m}$ fixture are the shortest in duration, while the $0.10 \mathrm{~m}$ and $0.20 \mathrm{~m}$ fixtures produce plasmas which are comparable in lifetime. 


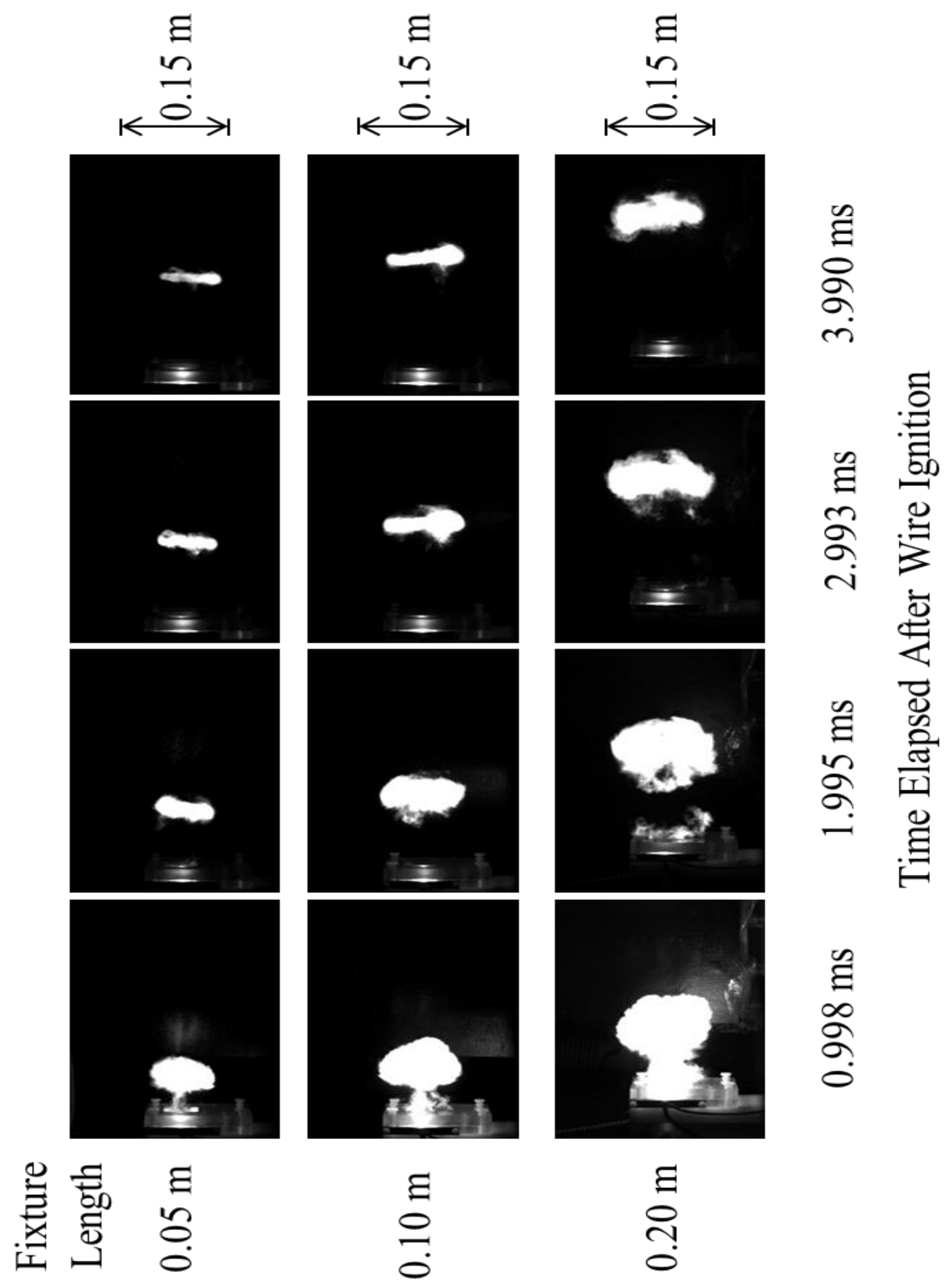

Figure 6.2 High speed images of TAP discharges from the alternative-length experiments 


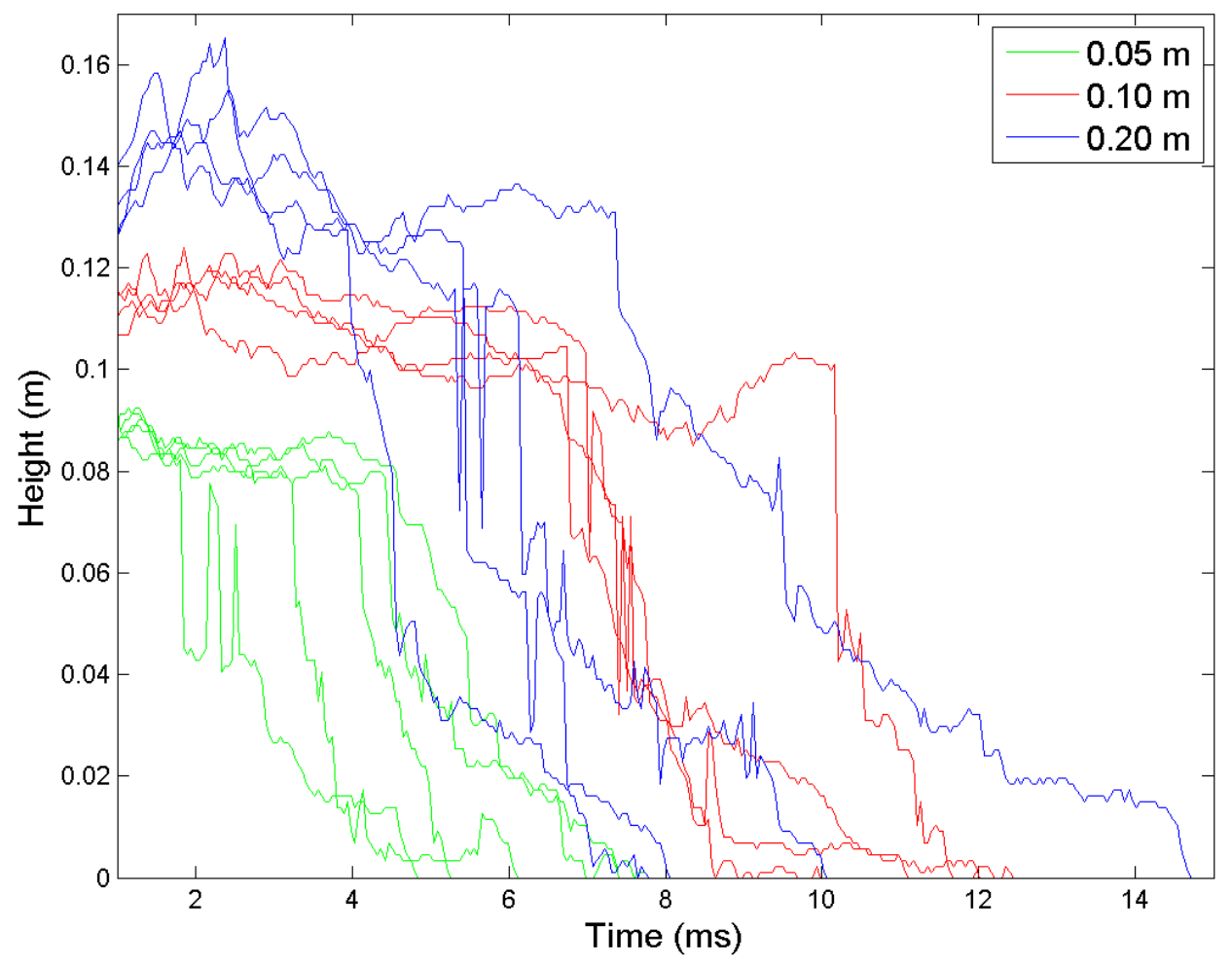

Figure 6.3 Comparison of height data of TAP discharges from alternative-length fixtures

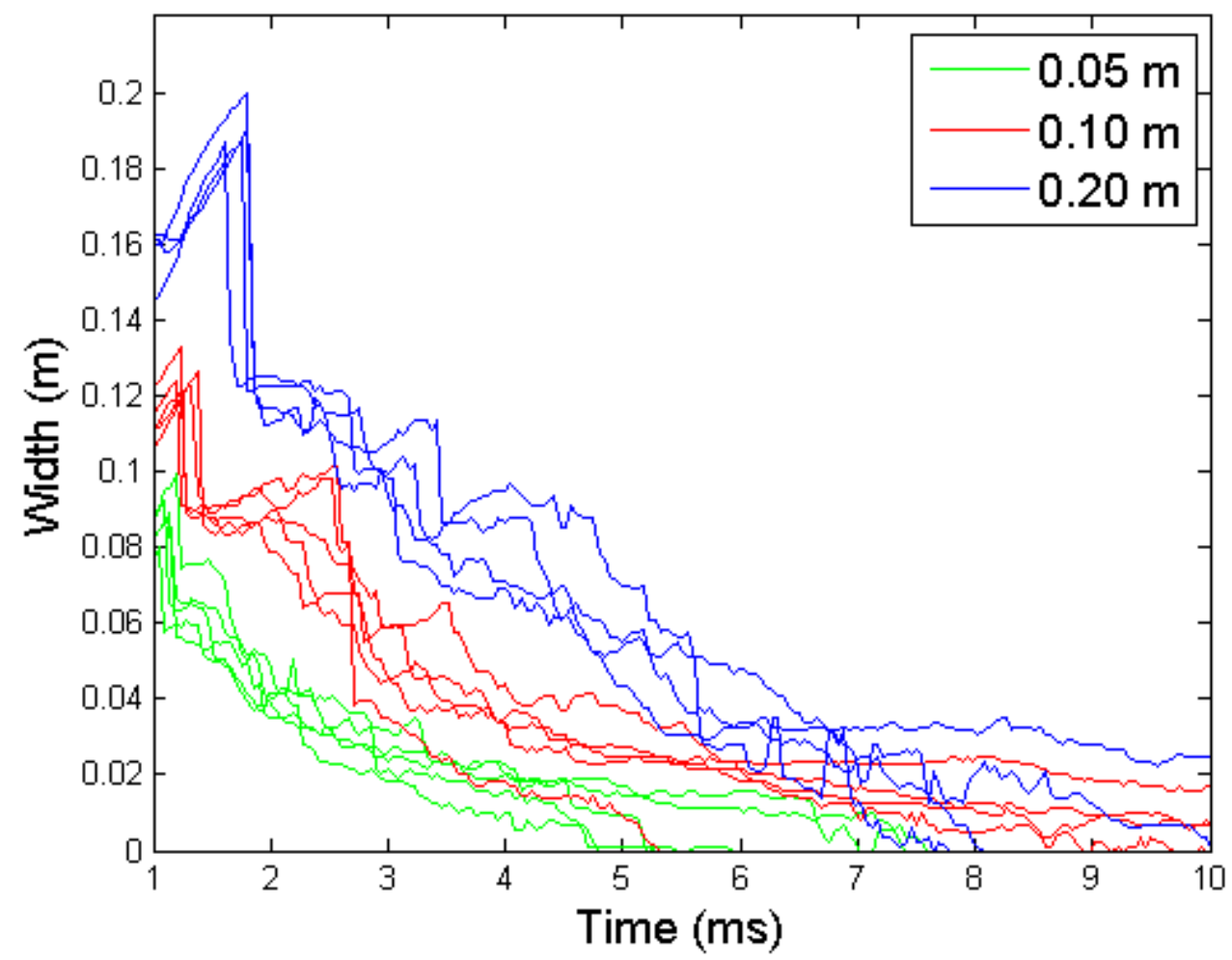

Figure 6.4 Comparison of width data of TAP discharges from alternative-length fixtures 


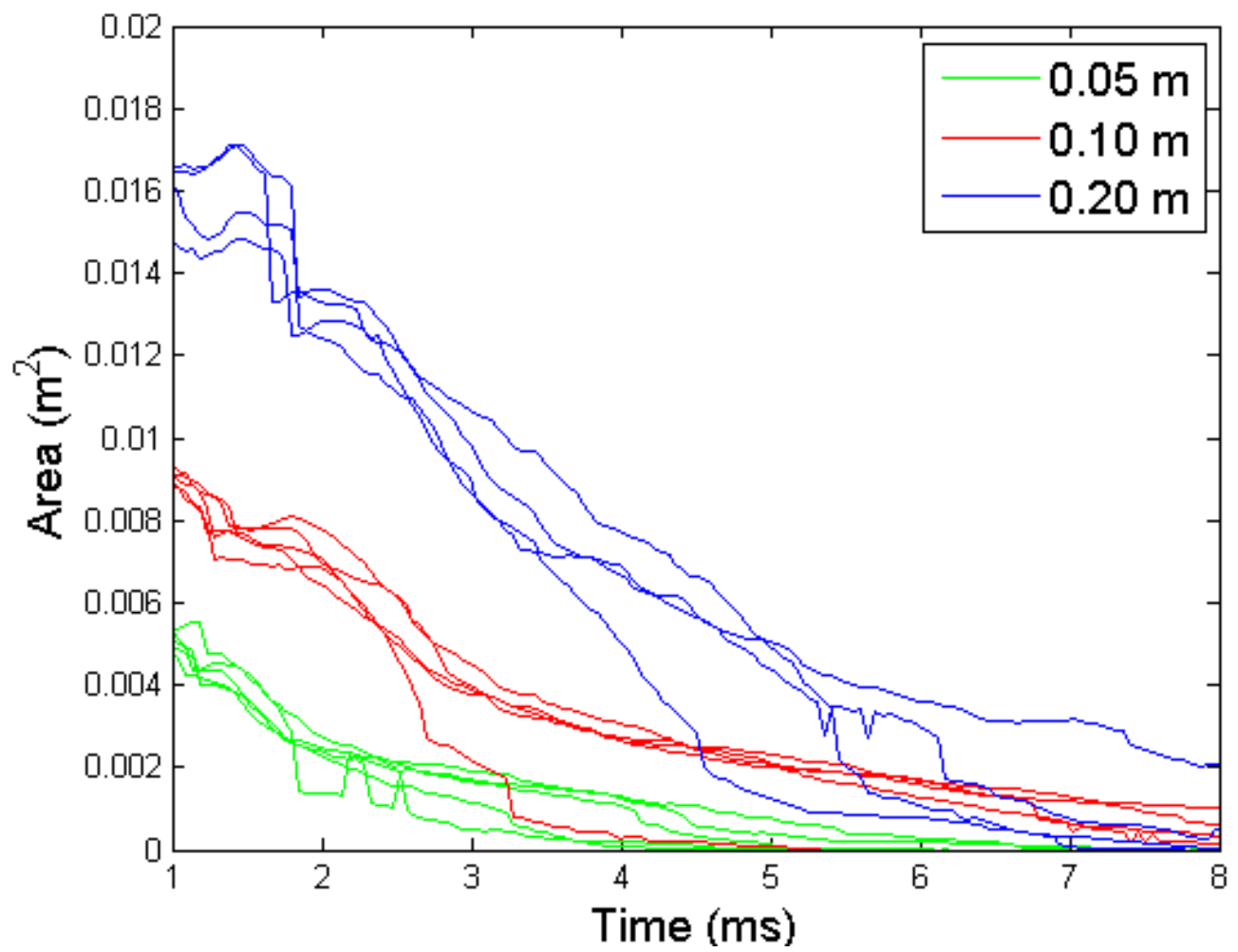

Figure 6.5 Comparison of area data of TAP discharges from alternative-length fixtures

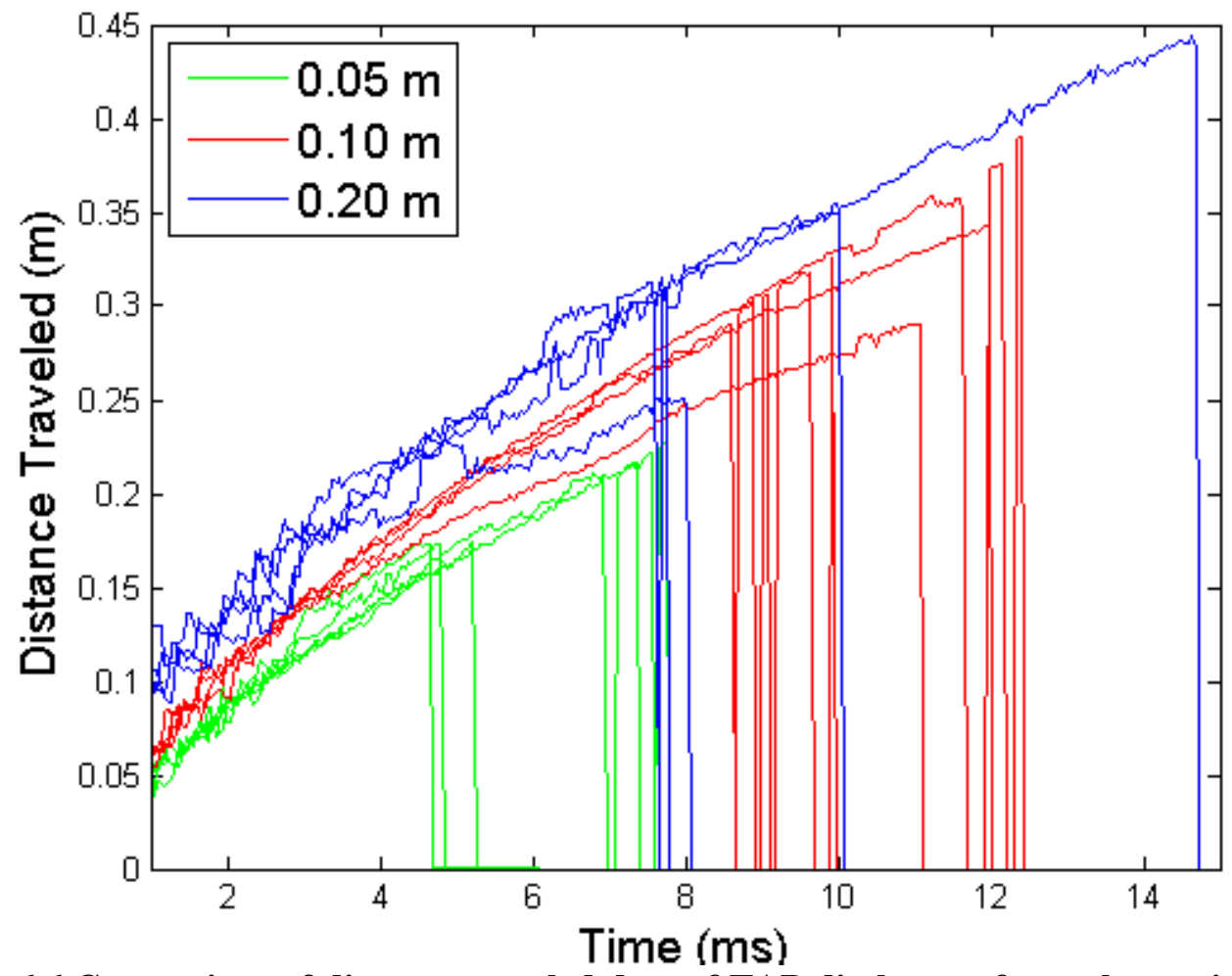

Figure 6.6 Comparison of distance traveled data of TAP discharges from alternative-length fixtures 
Figure 6.7 is the average area of the TAP discharges from these experiments. On average, doubling the length of the fixture more than doubles the area of the TAP discharge. Additionally, the $0.10 \mathrm{~m}$ and $0.20 \mathrm{~m}$ test fixtures result in TAP discharges which are approximately 70\% longer in duration and travel $62 \%$ farther than those from the $0.05 \mathrm{~m}$ fixture.

These experiments would suggest that a longer plasma fixture would produce an even larger plasma discharge, but would not significantly affect plasma duration. As potential applications for the TAP are explored, this conclusion should help maximize the plasma's size.

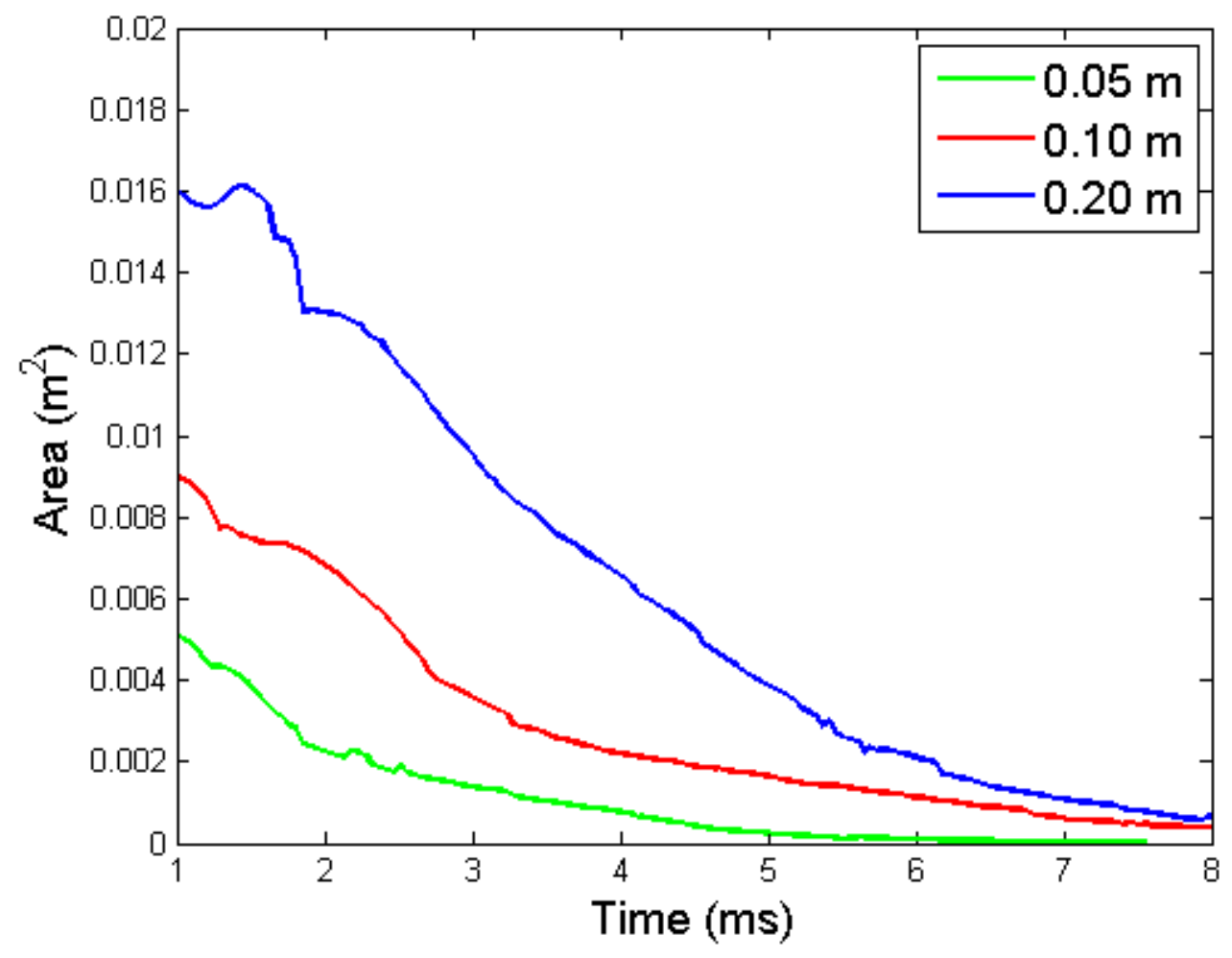

Figure 6.7 Comparison of the average area of TAP discharges from alternative-length fixtures 


\subsection{Multi-Tube Plasma Fixture}

In an attempt at scaling up the plasma discharge, four tubes were added to the plasma fixture. Each tube was wired in parallel using the same electrical connections without resistive or inductive isolation circuitry. A single wire was connected from the anode to the cathode through each tube. This configuration was an attempt to ignite several wires in parallel in isolated tubes with the hypothesis that multiple discharges could occur and increase plasma volume. Figure 6.8 shows a photograph of the plasma fixture with multiple tubes for these experiments.

Two experimental trials were conducted with this configuration. The resultant discharge, shown in Figure 6.9, was very different than the typical TAP. Comparing Figure 6.9 to Figure 5.4, the discharge from the multi-tube plasma fixture is seen to be significantly smaller in size and duration than the prototypical configuration. As from before, it is important to recognize the stationary discharge shown in the middle of each image as a separate discharge from the detached plasma.

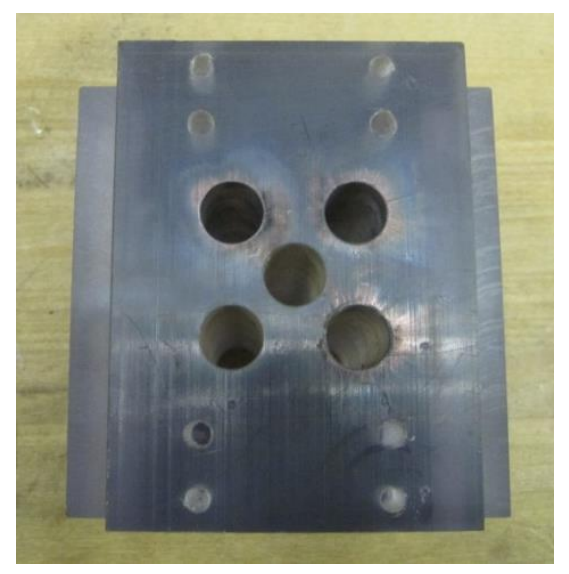

Figure 6.8 Multi-tube plasma fixture 

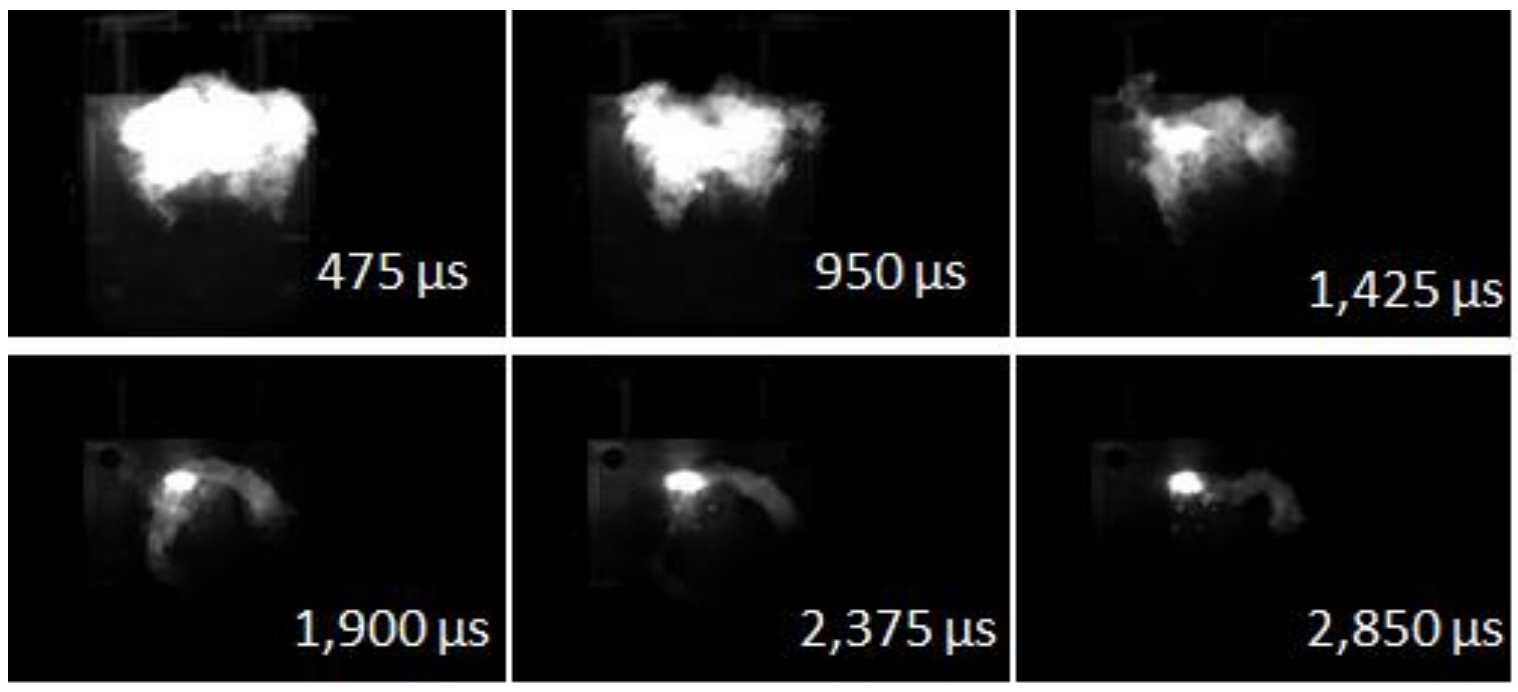

Figure 6.9 On axis images of discharge from the multi-tube plasma fixture

Additionally, experimental evidence showed that only one tube ignited out of the five tubes. This was evident in the carbon deposit pattern left behind on the plastic liners in each tube of the test fixture. This occurred in both trials. It is clear from the results that once a wire starts to heat and expand, it will explode and discharge all the energy rather than allowing the other wires to heat and ignite. In future experiments it should be possible to electrically isolate each tube with a redesigned driving circuit.

\subsection{Series-Wire Fixture Experimentation}

In another attempt at creating parallel TAP discharges, a new fixture was designed and fabricated that had a single length of wire connecting four tubes in series. The test fixture, shown in Figure 6.10, was designed to link each tube in series. This was a significant alteration to the geometry of the configuration of Section 3.3. The high voltage and ground connections are both at the sealed back end of the fixture. The front of the fixture consists of one screen for a top pair of tubes, and one screen for the bottom pair of tubes. The left image of Figure 6.11 shows the front of the fixture where the plasma is allowed to expand out of the chamber's four tubes. The center image shows the 
back of the fixture, and the right image shows the brass plates that are at three separate electric potentials, separated by the fixture itself. The top and bottom sets of holes are further separated in the front by the polycarbonate plasma fixture.
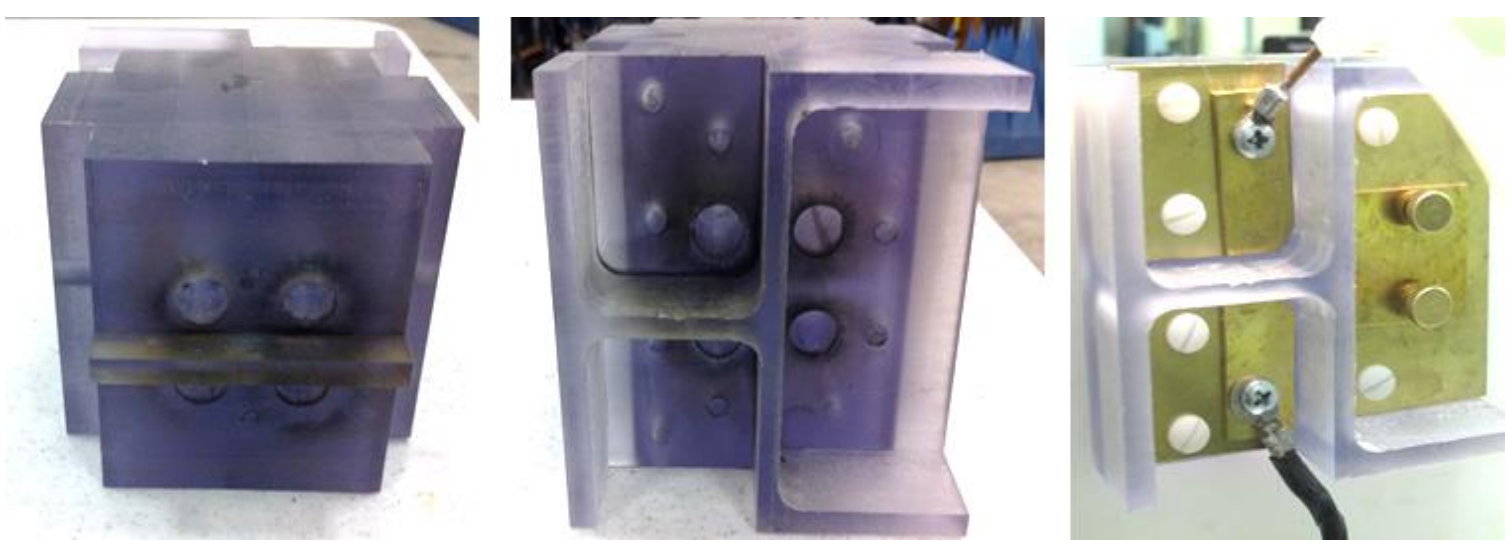

Figure 6.10 Series-wire fixture, front (left), back (center), back with connections (right)

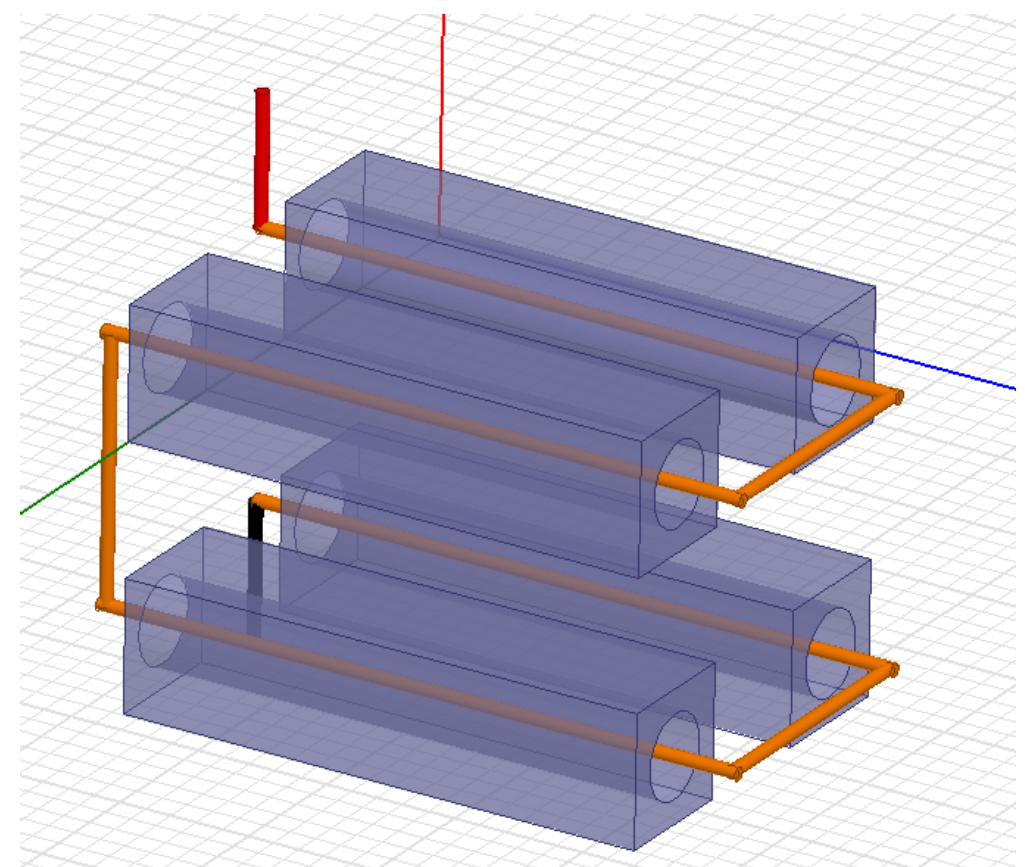

Figure 6.11 Wiring diagram for the series-wire fixture. The wire is shown orange, with the high voltage connection shown red and the ground connection shown black

The wire is strung taut back and forth through the fixture down each tube, as shown in the wiring diagram of Figure 6.11, where the high voltage connection is colored red, and the ground connection is colored black (the wire is colored orange). Different from 
the typical configuration, the wire lengths at the front of the fixture have a non-zero potential. Due to this high potential at the front of the fixture, an accelerator grid was not mounted on the fixture. If an accelerator grid were placed in its typical configuration, then the high voltage front wires would arc to the $250 \mathrm{~V}$ accelerator grid altering the fundamental conditions for the TAP to form.

Experiments with this series-wire fixture proved to ignite all four tubes consistently. The resultant discharge is similar in effect to the TAP. The discharge detaches from the fixture and forms into a toroid as it travels, as shown in Figure 6.12, an image of the discharge as recorded by the SVSI high speed camera 2,345 $\mu$ s after vaporization. Another image of the discharge, Figure 6.13, as recorded by the Phantom high speed camera $380 \mu \mathrm{s}$ after vaporization, depicts that multiple tubes ignite during the early formation of this discharge.

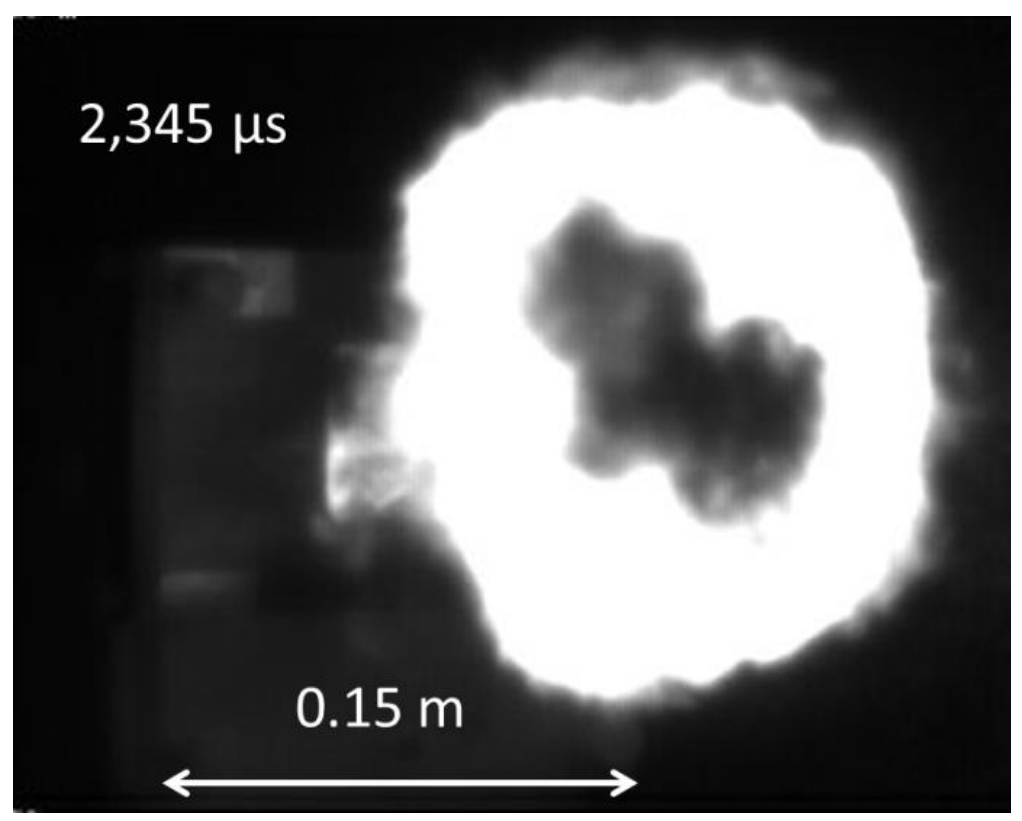

Figure 6.12 Image of the toroidal discharge from the series-wire fixture (scale and timing are shown) 


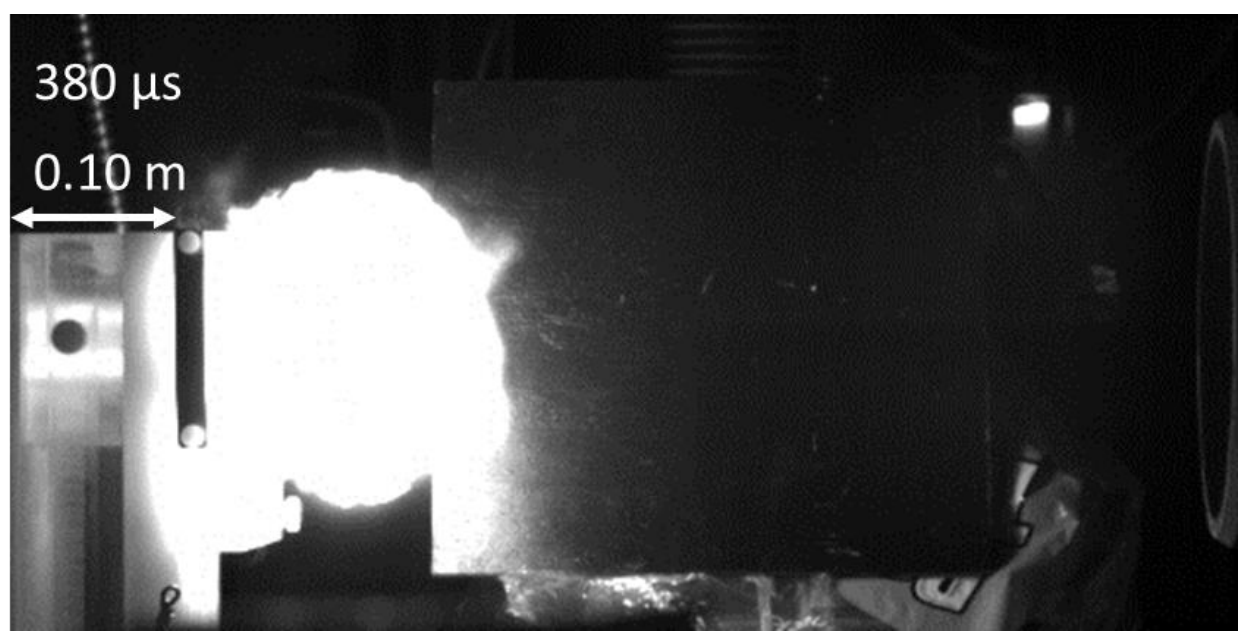

Figure 6.13 Image of the discharge from the series-wire fixture (scale and timing are shown)

The videos of the discharges as recorded by the Phantom high speed camera were analyzed using the image processing algorithm, and the results were compared to those from the prototypical experiments. The following graphs compare the height, width, area, and distance traveled of the discharges over time for each of these experiments. The thin black traces are from videos of the series-wire fixture and the results from the nominal configuration, discussed in Chapter 5, are shown as the thick traces. Figure 6.14 is a comparison of the heights of the discharges from these experiments, Figure 6.15 compares the widths, Figure 6.16 compares the areas, and Figure 6.17 compares the distances traveled.

The geometry of this discharge changes many variables of the experiment compared to the nominal configuration. This fact makes it difficult to make concrete conclusions about the results. The series-wire fixture produces a discharge that is on average $80 \%$ larger in area for the first $1.5 \mathrm{~ms}, 25 \%$ shorter in duration, and $9 \%$ farther in distance traveled, resulting in plasmas that are $46 \%$ faster in velocity than those from the nominal fixture. An important fact to remember is that this experimental fixture lacks an acceleration region to enhance the discharge. If the fixture were redesigned to include an 
acceleration region then perhaps the resultant discharge would be even larger in size and endure longer.

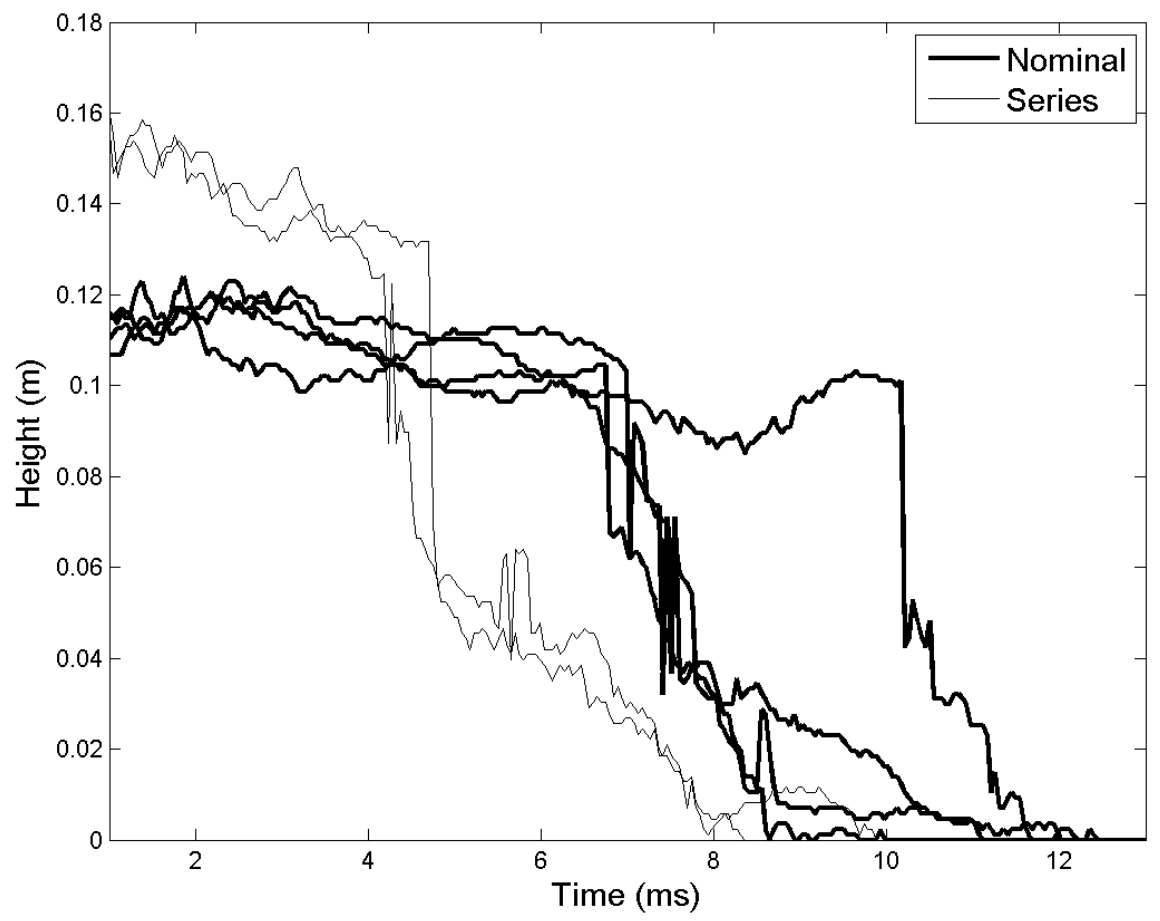

Figure 6.14 Comparison of height data of nominal TAP fixture and from the series-wire fixture

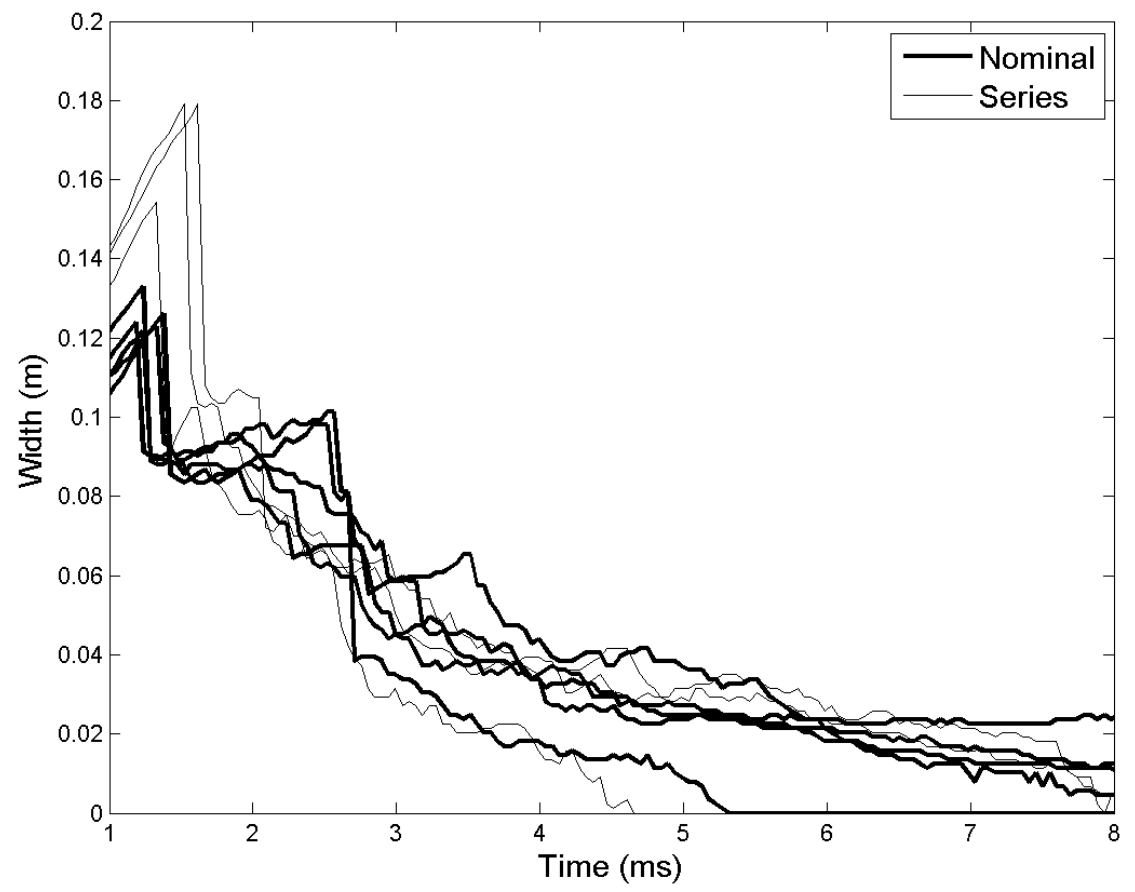

Figure 6.15 Comparison of width data of nominal TAP fixture and from the series-wire fixture 


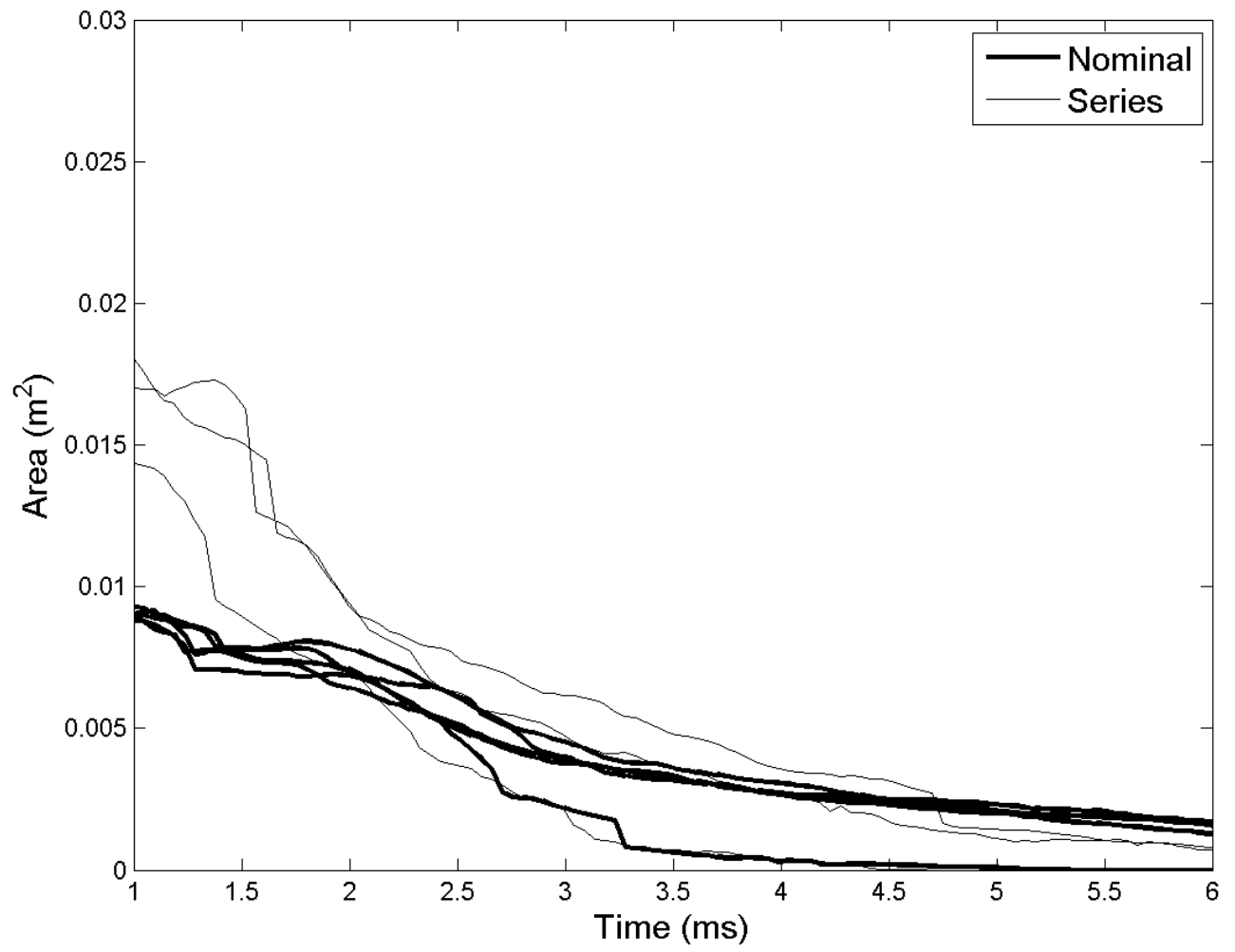

Figure 6.16 Comparison of area data of nominal TAP fixture and from the series-wire fixture

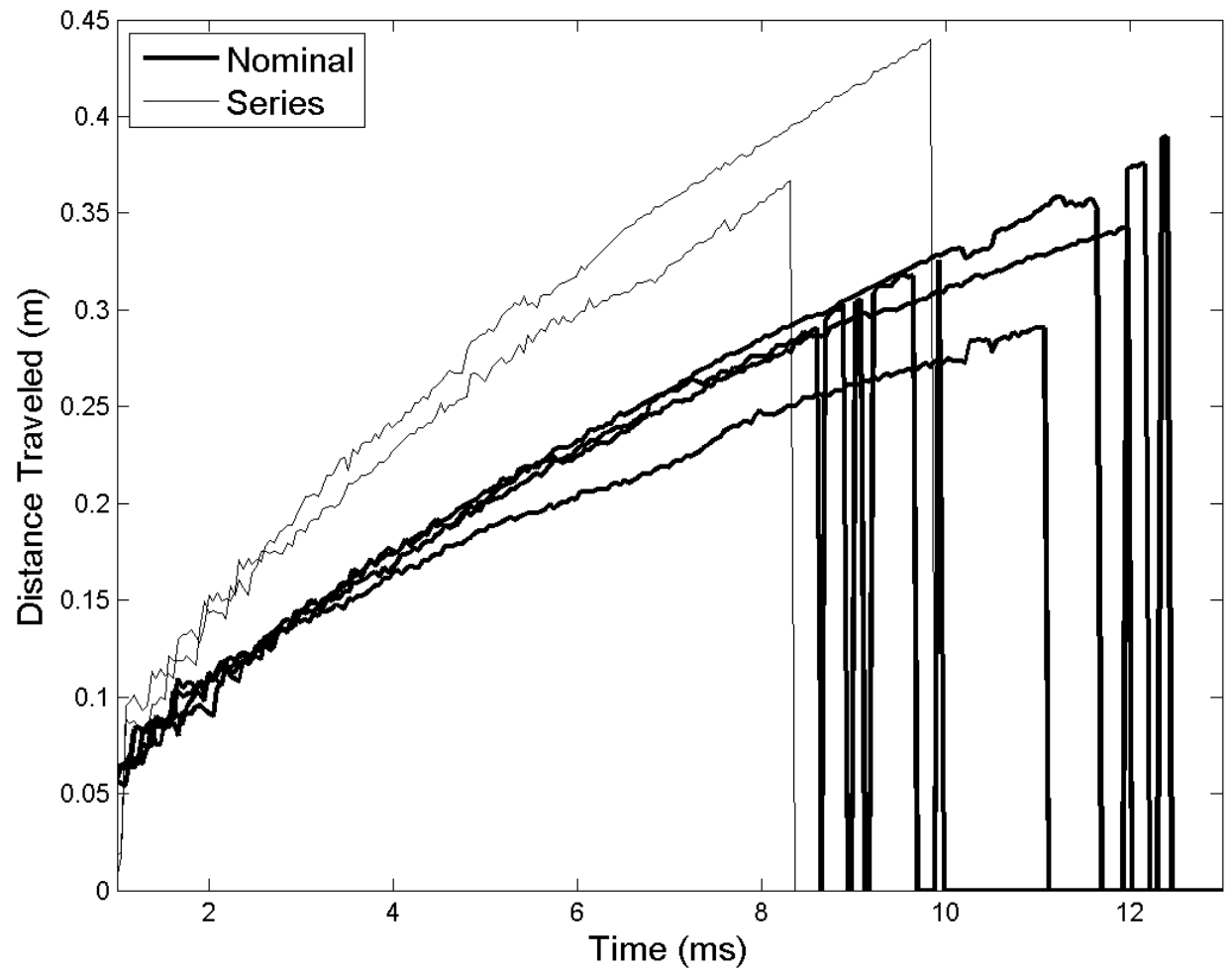

Figure 6.17 Comparison of distance traveled data of nominal TAP fixture and from the series-wire fixture 


\subsection{Energy Scaling Experiments}

A series of experiments were conducted to help understand how the TAP discharge scales with energy. Increasing the energy driven through the wire should increase the energy deposited into the discharge and could have a noticeable effect on the size of the TAP.

For these experiments, another five AeroVox high voltage capacitors were added to the circuit. These additional capacitors were each $2.2 \mu \mathrm{F}$, for a new total capacitance of $22 \mu \mathrm{F}$. All other experimental conditions and circuit parameters remained the same as in the typical configuration outlined in Chapter 3. The larger capacitor bank was charged to $30 \mathrm{kV}$, and the stored energy of $8.8 \mathrm{~kJ}$, formerly $4.4 \mathrm{~kJ}$, was used to drive the wire load in the same manner as all other experiments.

Five experiments were conducted with the higher stored energy. The resultant discharges were recorded by the Phantom v7.3 high speed camera and the video files were analyzed using the image processing algorithm. The discharges increased in height, width, area, and distance traveled when compared with plasmas formed by the nominal $4.4 \mathrm{~kJ}$ stored energy configuration.

The following graphs compare the height, width, area and distance traveled of the discharges over time for each of these experiments. The thick black traces are from videos of the increased energy storage, and the results from the nominal $4.4 \mathrm{~kJ}$ configuration are shown as the thin traces. Figure 6.18 is a comparison of the heights of the discharges from these experiments, Figure 6.19 compares the widths, Figure 6.20 compares the areas, and Figure 6.21 compares the distances traveled. The increased 
energy storage resulted in TAP discharges which were on average 93-177\% larger in area, $27 \%$ longer in duration, and traveled $27 \%$ farther than the typical configuration.

These experiments show that the TAP discharge does increase in size and duration with increased energy deposited. While the stored energy was doubled, these experiments were conducted with the voltage and current probes monitoring a different part of the circuit, not the exploding wire, thus these experiments would need to be repeated in order to obtain an accurate measurement of the voltage and current through the plasma to be able to calculate the energy deposited into the plasma.

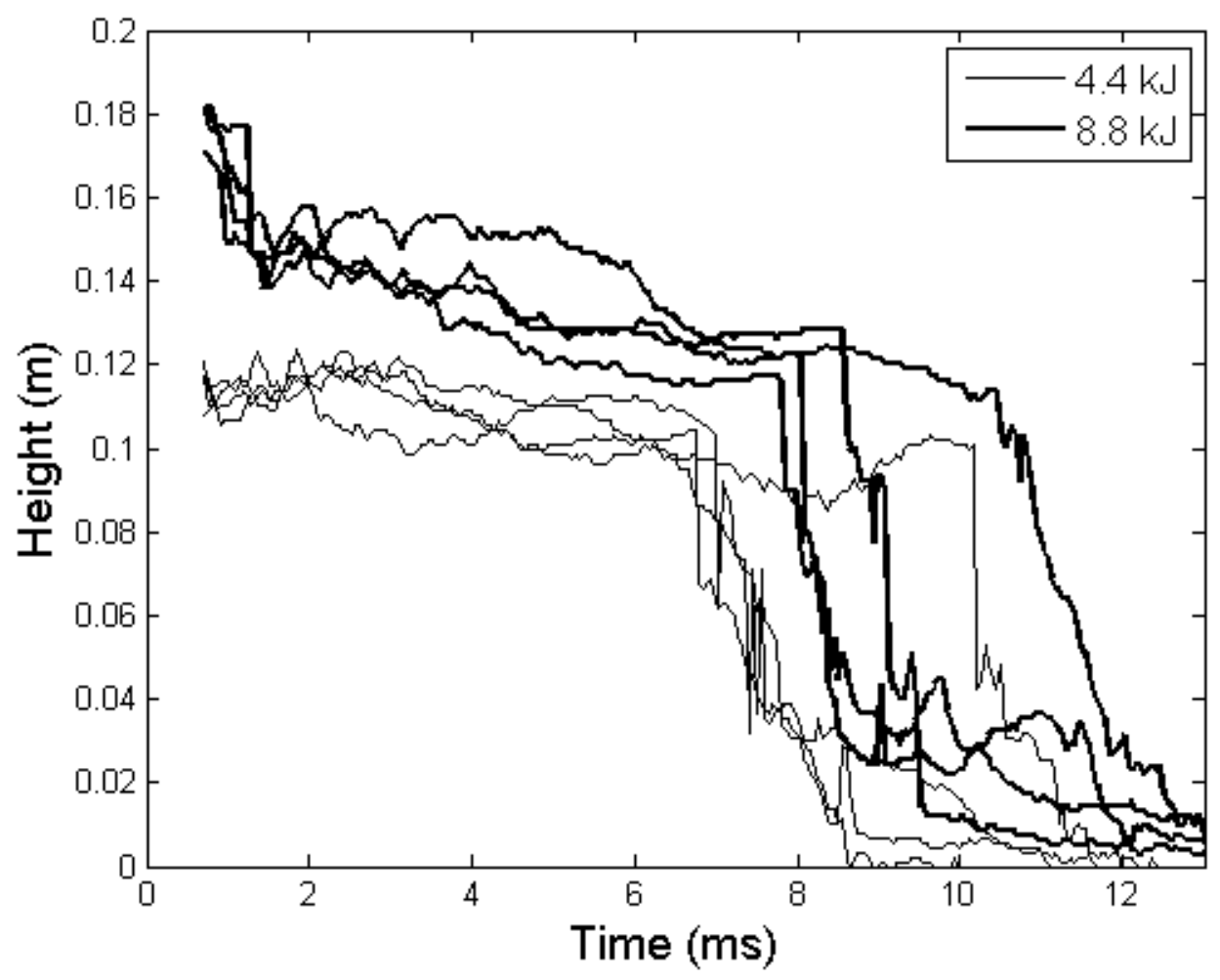

Figure 6.18 Height data of increased energy experiments compared with data from the nominal experiments 


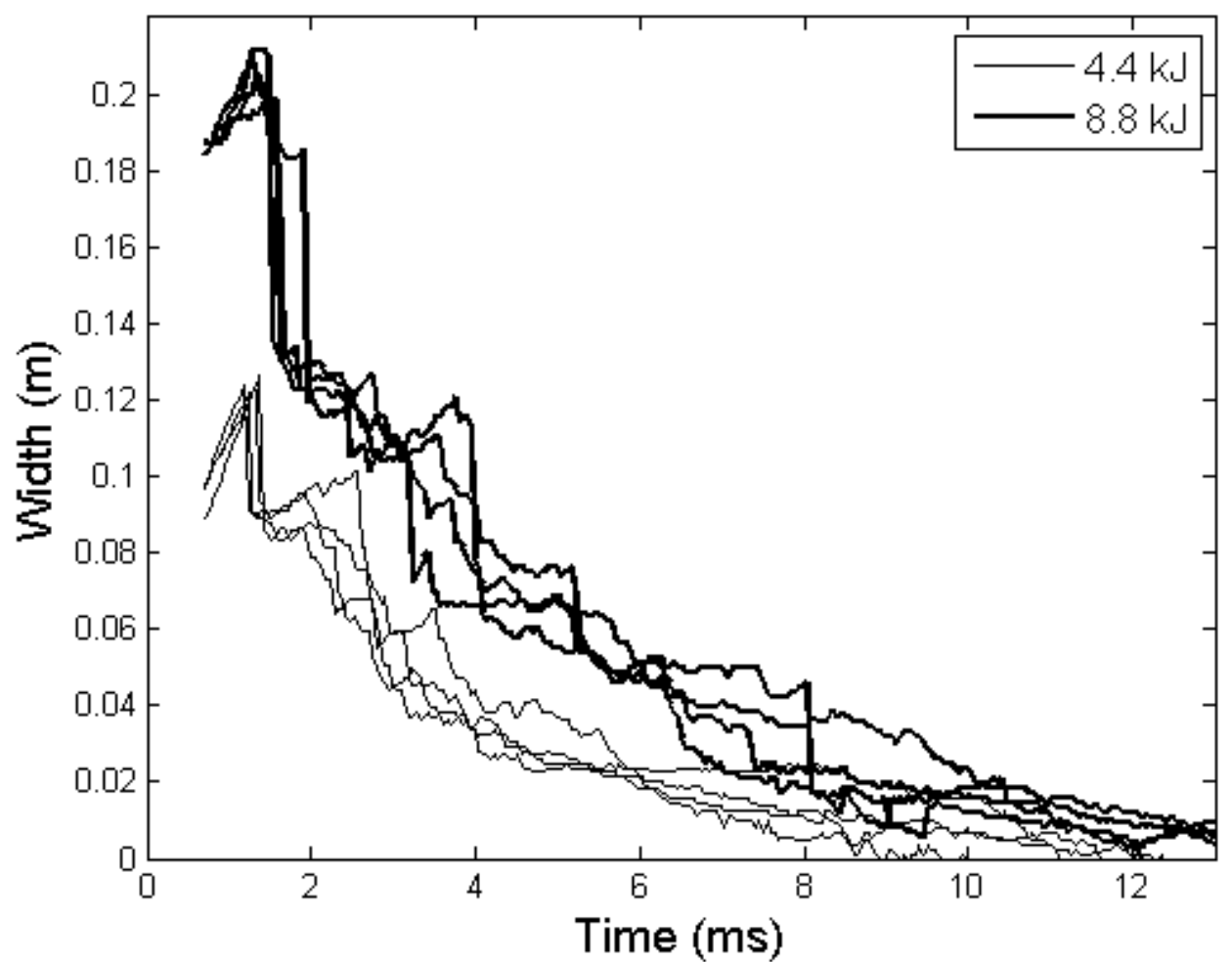

Figure 6.19 Width data of increased energy experiments compared with data from the nominal experiments

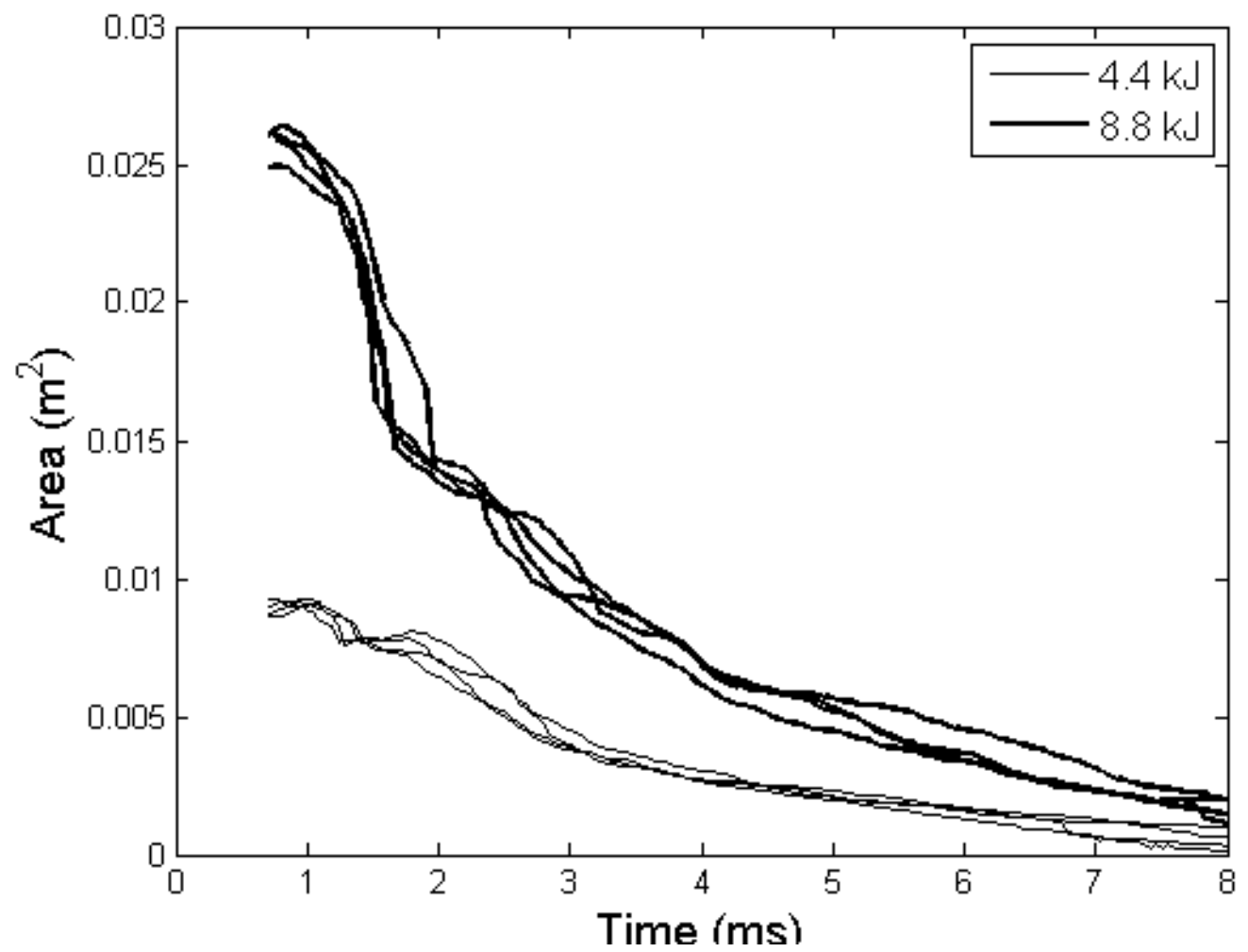

Figure 6.20 Area data of increased energy experiments compared with data from the nominal experiments 


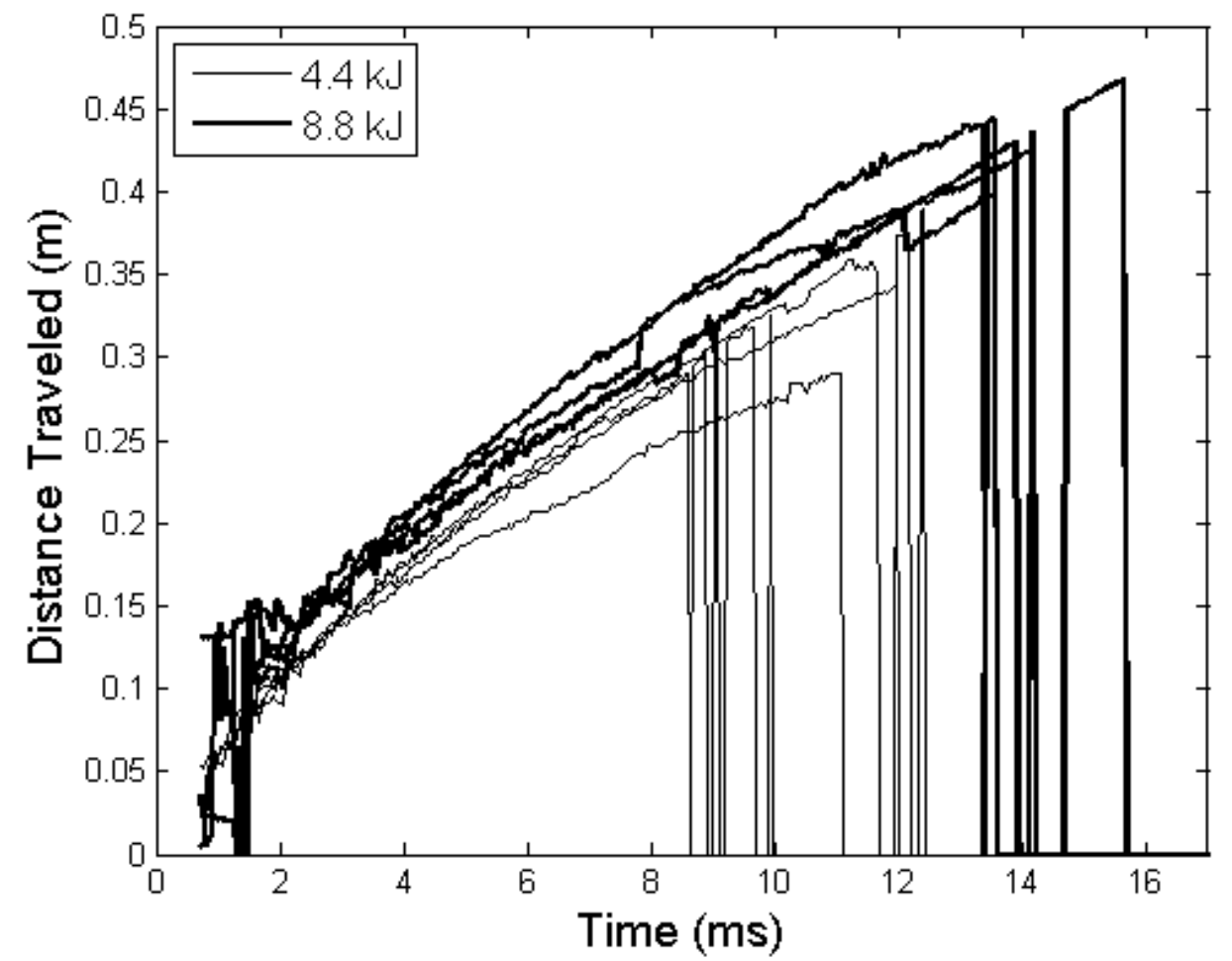

Figure 6.21 Distance traveled data of increased energy experiments compared with data from the nominal experiments

\subsection{Varying of the Load and Other Circuit Parameters}

Circuit inductance, circuit resistance, and the material properties of the wire load are all critical parameters in the process resulting in the vaporization of the wire as discussed in Chapter 2 and Appendix A. Each parameter can affect the action integral, the restrike of the wire load, and the energy deposited into the load. Experiments were conducted to analyze if changing these parameters in the plasma circuit would effect the formation and evolution of the TAP discharge. A portion of this data has been published in [1].

\subsubsection{Wire Material}

To investigate the effect of the wire load on the evolution of the TAP discharge, a series of experiments were conducted with six different wire material and thickness combinations. The wire materials tested were the prototypical 40 AWG silver plated 
copper wire, bare copper wire with thickness 30 AWG and 40 AWG, nickel chromium wire of 28 AWG and 40 AWG, and tungsten wire of 36 AWG. These materials were chosen for availability and variety. Separate experimental trials were conducted using each of the six wire materials.

The discharge created from each was observed with the Phantom 7.3 high speed camera to compare to the nominal TAP discharge (created with a silver plated $\mathrm{Cu}$ 40 AWG wire). The discharges were analyzed using the video processing algorithm discussed in Section 4.3 and Appendix C.

A comparison of the distance traveled data from the $\mathrm{Cu}$ wire experiments is given in Figure 6.22. The $30 \mathrm{AWG}$ (thin solid black lines) was shown to produce a discharge which is $35 \%$ smaller in area on average and are $159 \%$ shorter in duration than those of the typical wire (Cu-Ag 40 AWG). The two 40 AWG wires, one with a $6 \%$ silver plating and one without, produced comparable plasma discharges.

A comparison of the area data of the discharges from the tungsten, nickel chromium, and the silver plated copper wire is shown in Figure 6.23. The 28 AWG nickel chromium wire produced discharges with the largest area. These discharges were on average $154 \%$ larger at $1 \mathrm{~ms}$ beyond vaporization, 261\% larger at $2 \mathrm{~ms}, 326 \%$ larger at $3 \mathrm{~ms}$, and $463 \%$ larger at $4 \mathrm{~ms}$, than those from the $\mathrm{Cu}-\mathrm{Ag}$ wire. Despite this increase in size, discharges from the $\mathrm{NiCr} 28 \mathrm{AWG}$ wire were only a modest $25 \%$ longer duration and traveled $18 \%$ farther. 


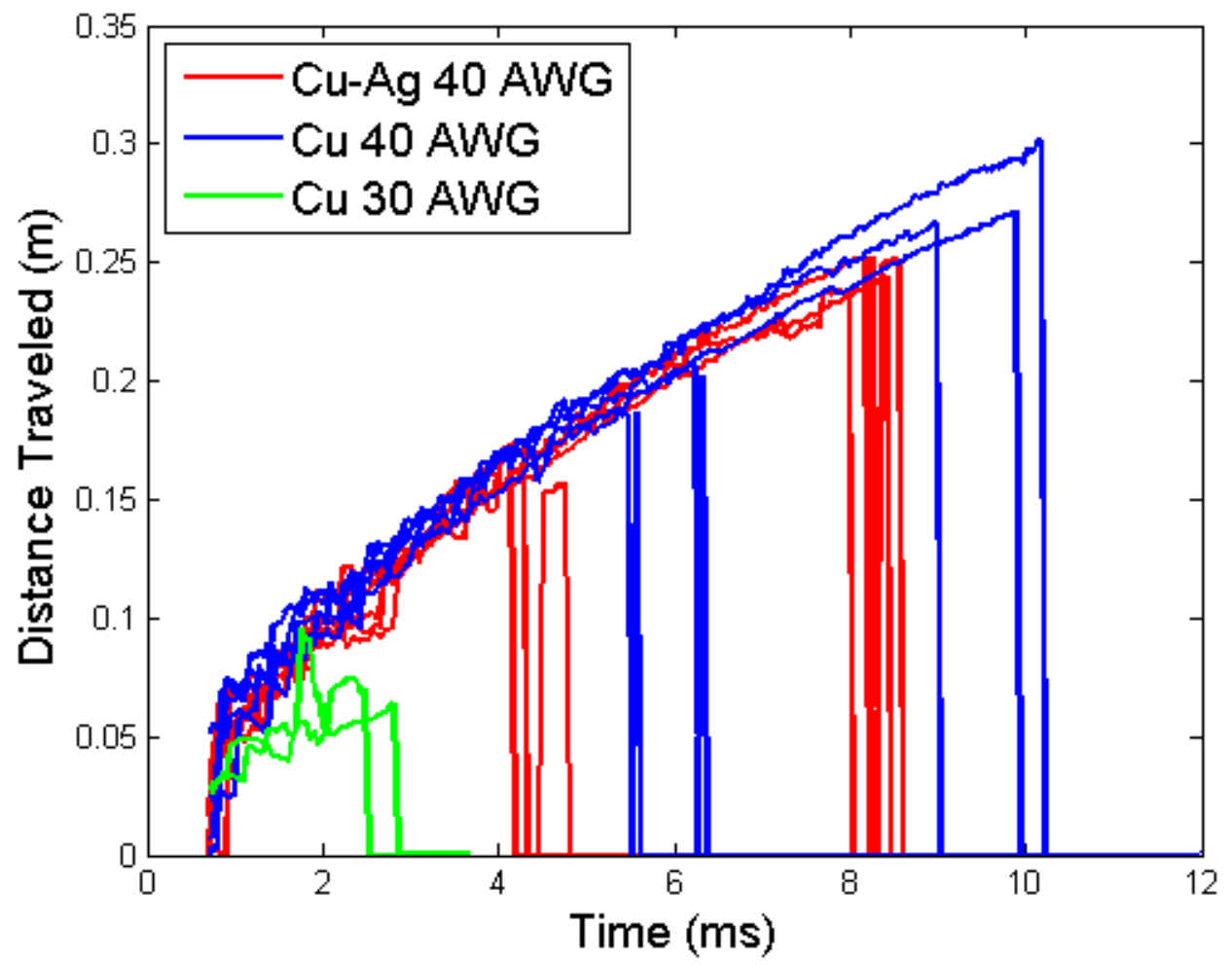

Figure 6.22 Comparison of distance traveled data from different copper wire loads

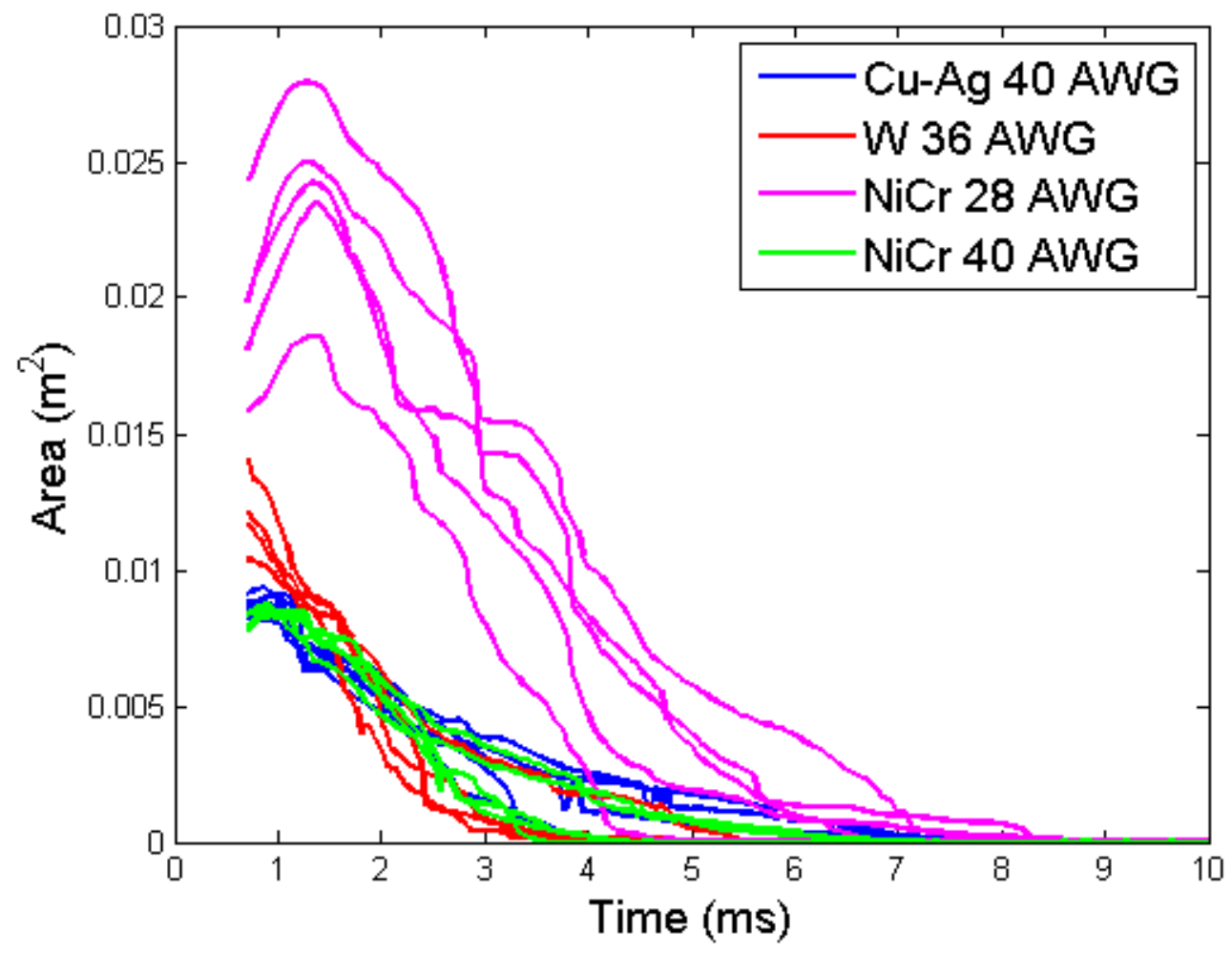

Figure 6.23 Comparison of area data from different wire materials 
It is difficult to draw all-encompassing conclusions from this data. Comparing the different thickness copper wire data would suggest that as the thickness of the wire increases, more of the energy from the circuit is lost in the transition of the additional material. However, this is not the case when comparing the $\mathrm{NiCr}$ wire data, as the 28 AWG wire produced a much larger discharge than the 40 AWG wire. It is important to note that it was difficult to solder the $40 \mathrm{AWG} \mathrm{NiCr}$ wire to the stainless steel high voltage cathode of the plasma fixture, and a poor electrical connection could have resulted in a smaller discharge. The important result of these experiments is that the prototypical silver plated 40 AWG copper wire was not the optimal material for producing the largest TAP discharge.

\subsubsection{Circuit Inductance}

A series of experiments were conducted to investigate the effect of the circuit inductance on the TAP discharge. The typical air core inductor of $11.77 \mu \mathrm{H}$ was replaced with inductors with inductances of $0.6 \mu \mathrm{H}$ and $27.5 \mu \mathrm{H}$. Five experimental trials were conducted with each condition.

Typical current measurements for the varying inductance experiments are shown in Figure 6.24. As shown, the differing inductance affected the peak and width of the current pulse. The $27.5 \mu \mathrm{H}$ inductor resulted in a current pulse delivered to the wire with a $5.48 \mathrm{kA}$ peak current, and a pulse width of $53.55 \mu \mathrm{s}$. The current pulse from the typical configuration had a peak of $6 \mathrm{kA}$ with a pulse width of $46.08 \mu \mathrm{s}$. The $0.6 \mu \mathrm{H}$ resulted in higher current delivered in a smaller pulse width, (6.88 kA, $35.9 \mu \mathrm{s})$. When comparing the three configurations, the current pulse decreased in amplitude and increased in pulse width as the inductance increased as expected from traditional circuit theory. 
Additionally, as shown in Figure 6.25, the current profile associated with restrike for the three inducatance values are different in timing and shape.

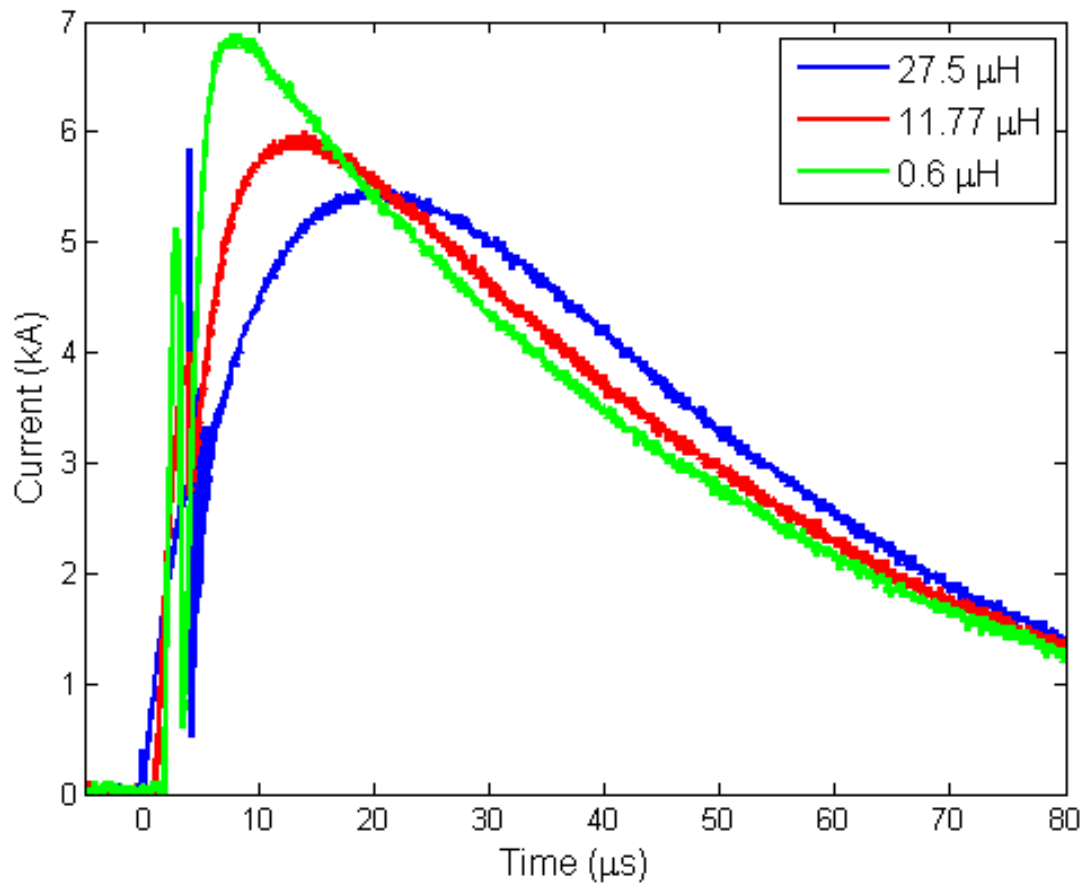

Figure 6.24 Current through the exploding wire for three inductance configurations

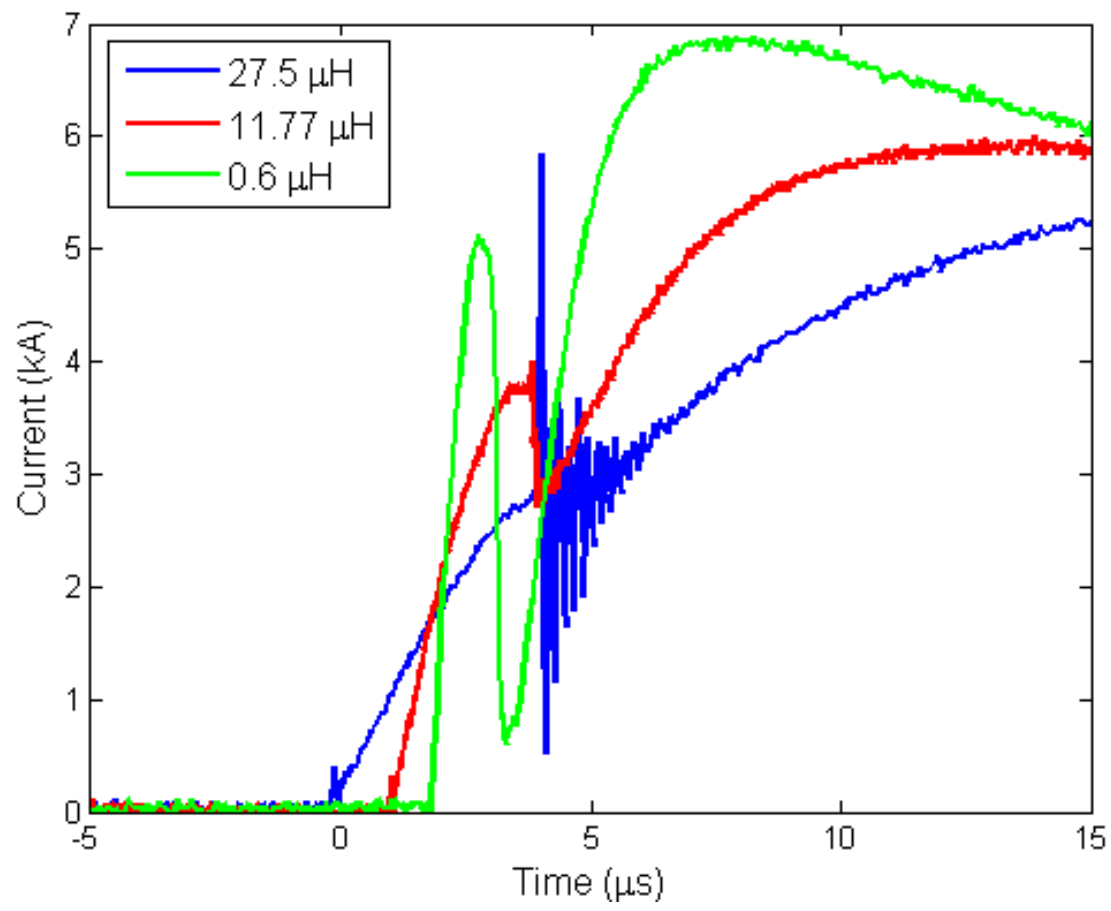

Figure 6.25 Expanded view of the current waveforms of Fig 6.24 
Figure 6.26 depicts the height data from these experiments as calculated by the image processing algorithm. The $27.5 \mu \mathrm{H}$ inductor data are shown as blue lines, the $11.77 \mu \mathrm{H}$ inductor data are shown as red lines, and the $0.6 \mu \mathrm{H}$ inductor data are shown as green lines. As shown, varying the circuit inductance did not result in any significant change in the height or duration of the TAP discharges. Similarly, no significant effect was observed in the distance traveled data.

Changing the circuit inductance from $0.6 \mu \mathrm{H}$ to $27.5 \mu \mathrm{H}$, (a $45 \mathrm{x}$ increase in inductance) resulted in a decrease in peak current of $20.3 \%$, and an increase in rise time of $34.6 \%$. This change in the amplitude and shape of the current pulse did not result in any observed change to the TAP discharge.

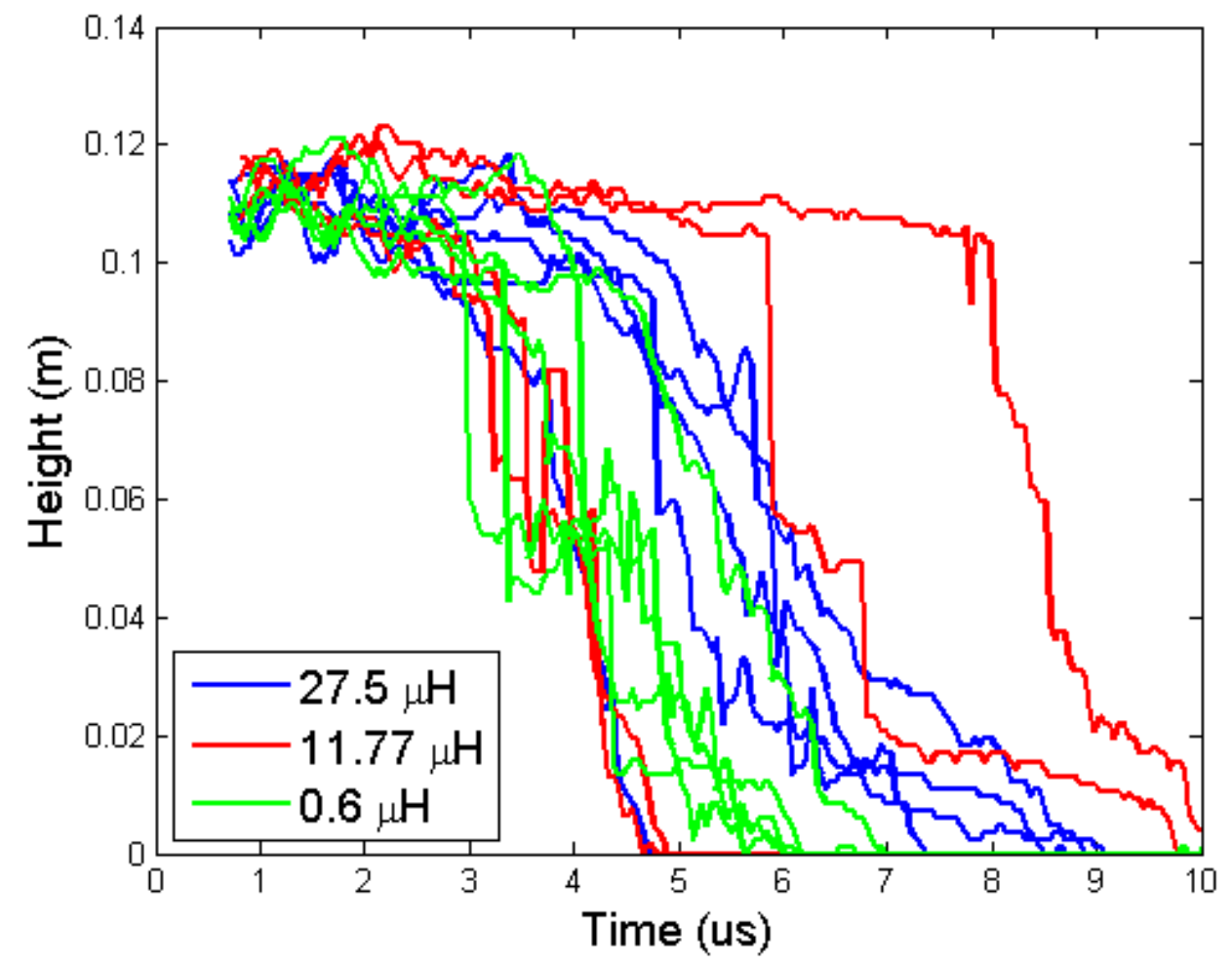

Figure 6.26 Height data of TAP discharges of varying circuit inductance. No significant change in the height, size, or duration of the TAP discharges were observed from altering the inductance value of the circuit. 


\subsubsection{Circuit Resistance}

A series of experiments were conducted to investigate the effect of varying the circuit resistance on the TAP discharge. To vary the circuit resistance, the aqueous-electrolyte resistor was changed from the typical $5.5 \Omega$ up to $20 \Omega$ and down to a negligible resistance (without an aqueous-electrolyte resistor) while holding the inductance at $11.77 \mu \mathrm{H}$.

The current waveforms for the $20 \Omega$ and $5.5 \Omega$ configurations are shown in Figure 6.27. As previously stated, the current pulse from the typical configuration $\left(\mathrm{R}_{1}=5.5 \Omega\right)$ had a peak of $6 \mathrm{kA}$ with a pulse width of $46.08 \mu \mathrm{s}$. The current pulse from the $20 \Omega$ circuit reached a peak of only $2.02 \mathrm{kA}$ with a pulse width of $130.85 \mu \mathrm{s}$.

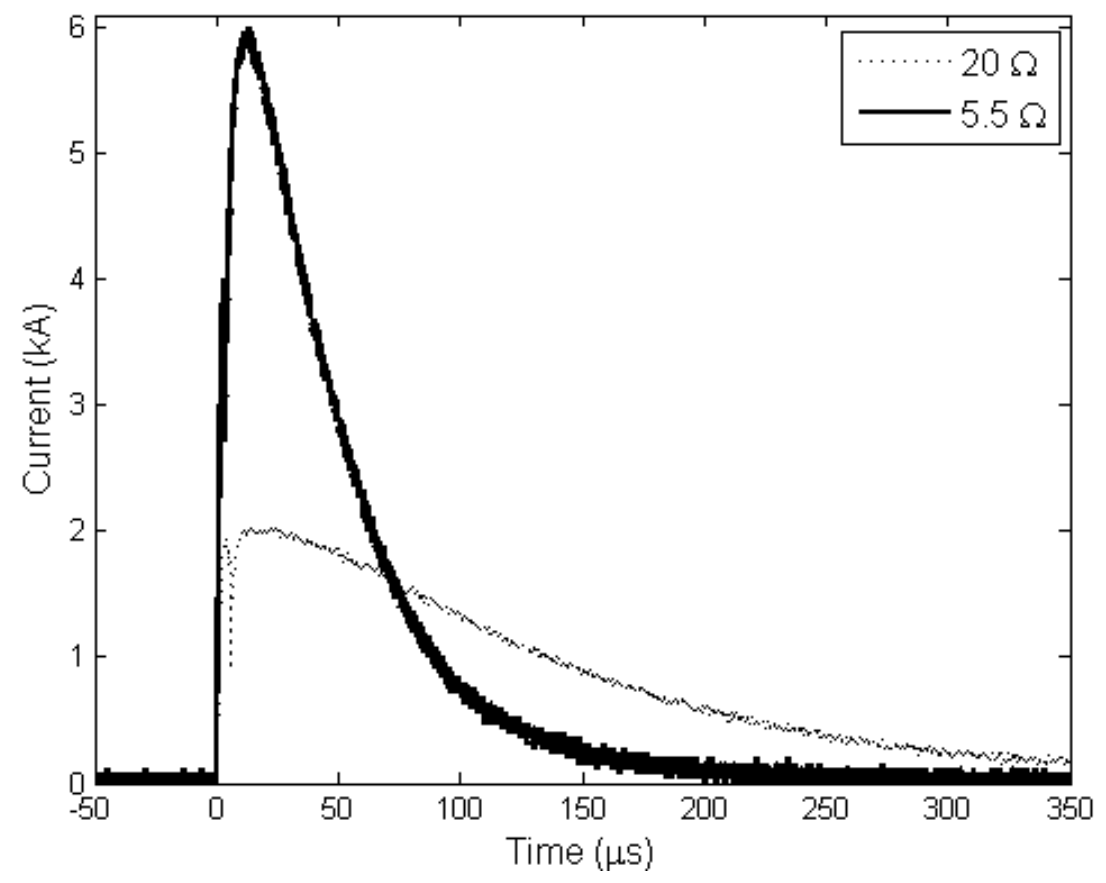

Figure 6.27 Current through the exploding wire for two resistor configurations

Removing the aqueous-electrolyte resistor from the circuit and directly connecting the inductor to the wire load resulted in a stray resistance of approximately $300 \mathrm{~m} \Omega$. This resulted in the circuit becoming underdamped, rather than the typical overdamped. The 
resultant current oscillates four times in $288 \mu \mathrm{s}$, reaching a peak of $23.6 \mathrm{kA}$, as shown in Figure 6.28.

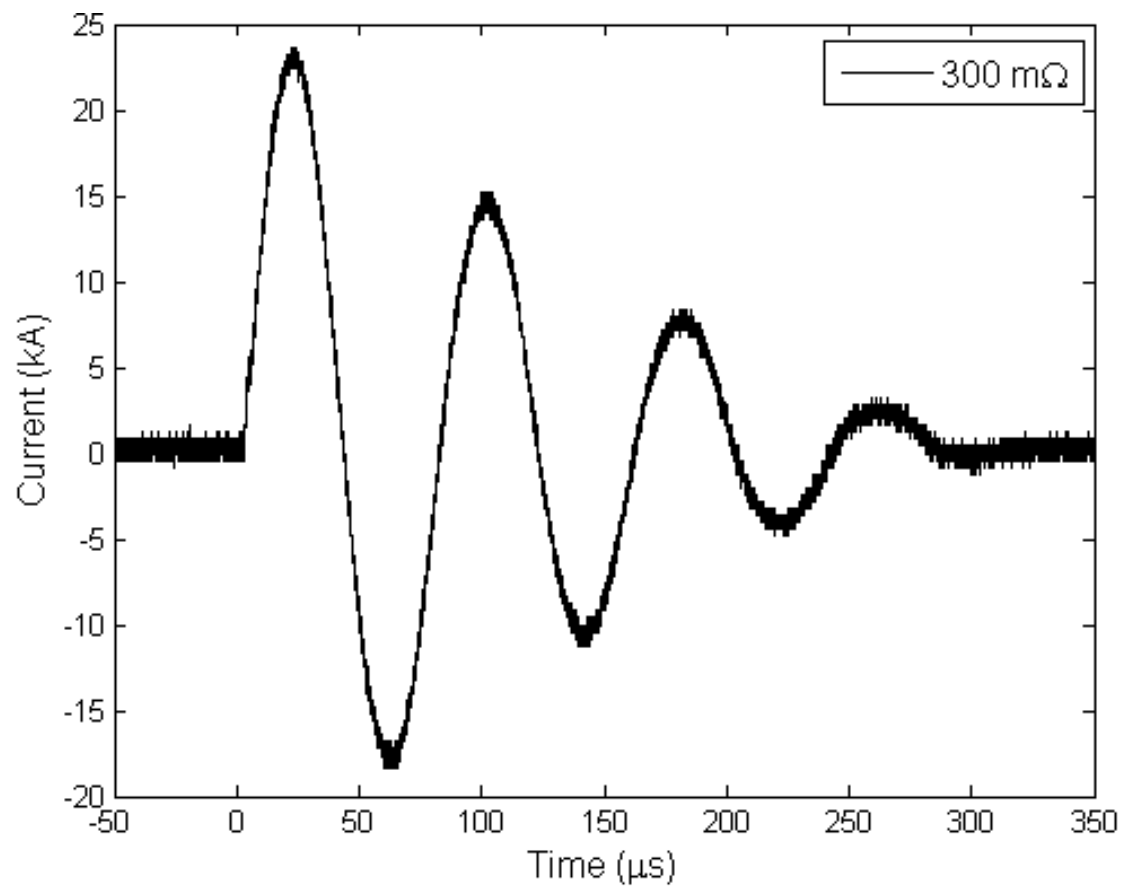

Figure 6.28 Current through the exploding wire for the circuit: $L_{1}=11.77 \mu H, R_{1}=300 \mathrm{~m} \Omega$

These changes yielded significant differences in the size and duration of the TAP discharge. Figure 6.29 is a comparison of the heights of the discharges from these experiments, Figure 6.30 compares the widths, Figure 6.31 compares the areas, and Figure 6.32 compares the distances traveled. The $20 \Omega$ resistor resulted in a shorter duration and smaller TAP when compared to the $5.5 \Omega$ configuration. This trend continued for the $300 \mathrm{~m} \Omega$ configuration to drastic effects. The TAP from the $300 \mathrm{~m} \Omega$ circuit was approximately twice as long in height and duration than those from the $5.5 \Omega$ circuit. Additionally, the $300 \mathrm{~m} \Omega$ discharges traveled approximately twice as far. 


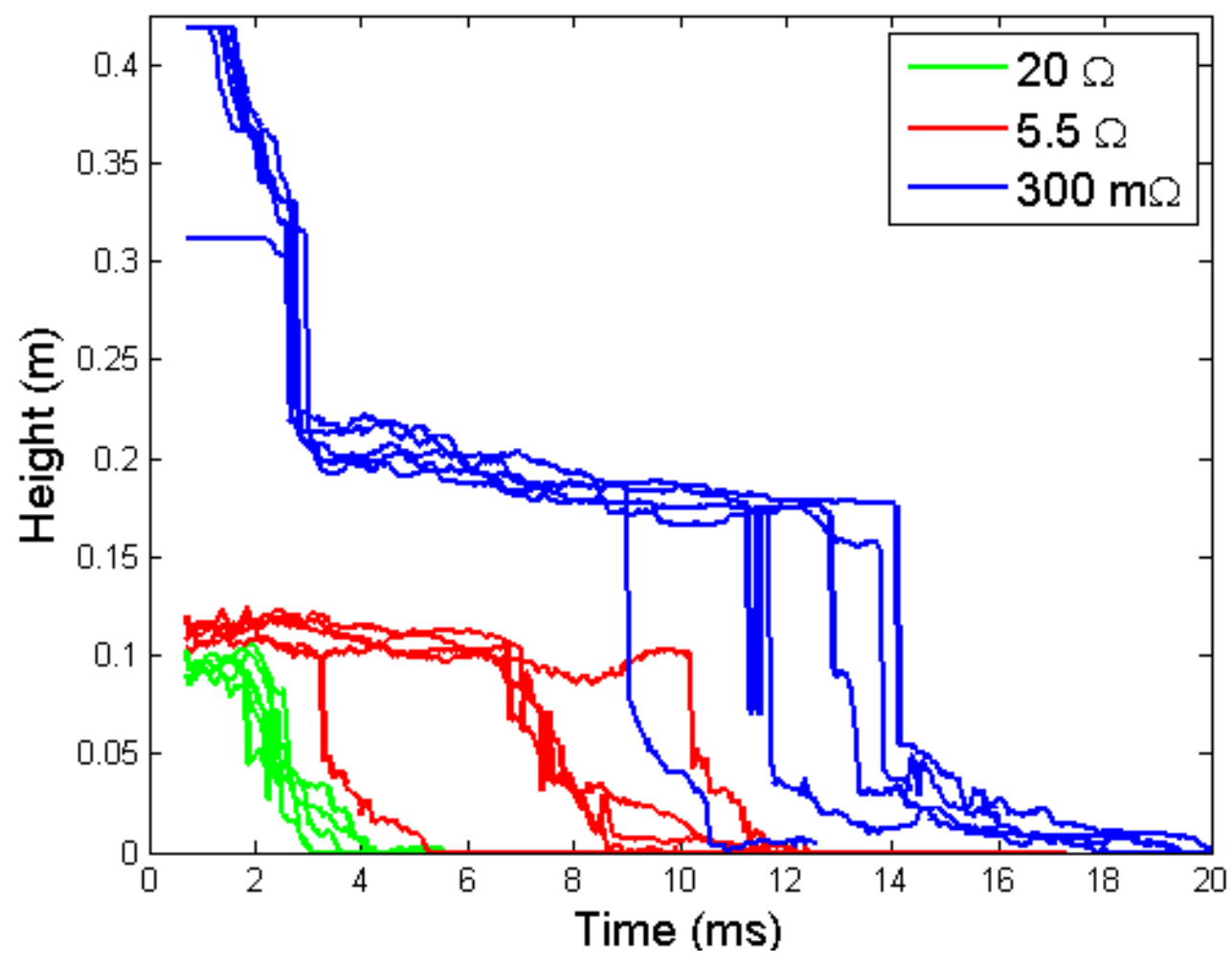

Figure 6.29 Height data of TAP discharges of varying circuit resistance

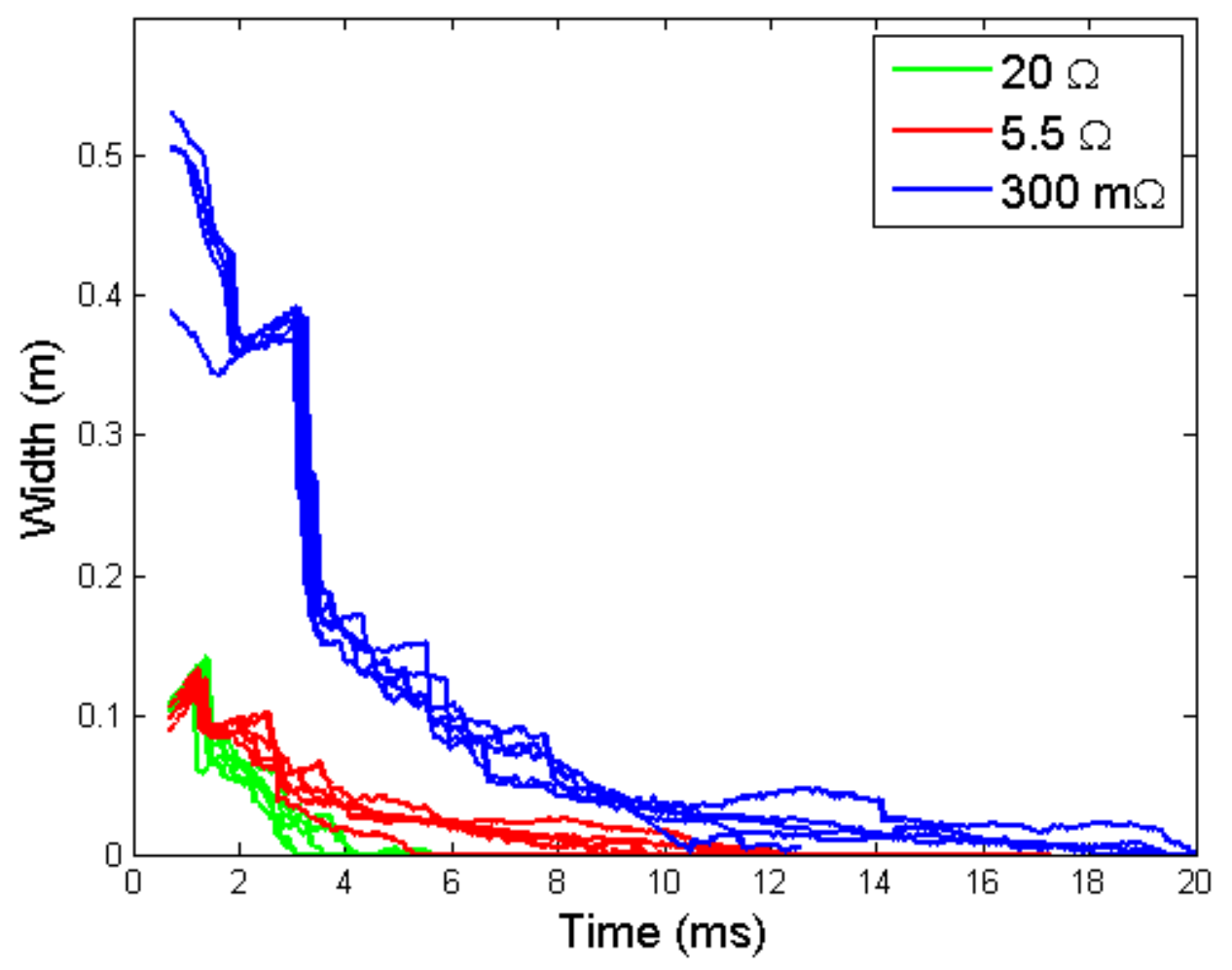

Figure 6.30 Width data of TAP discharges of varying circuit resistance 


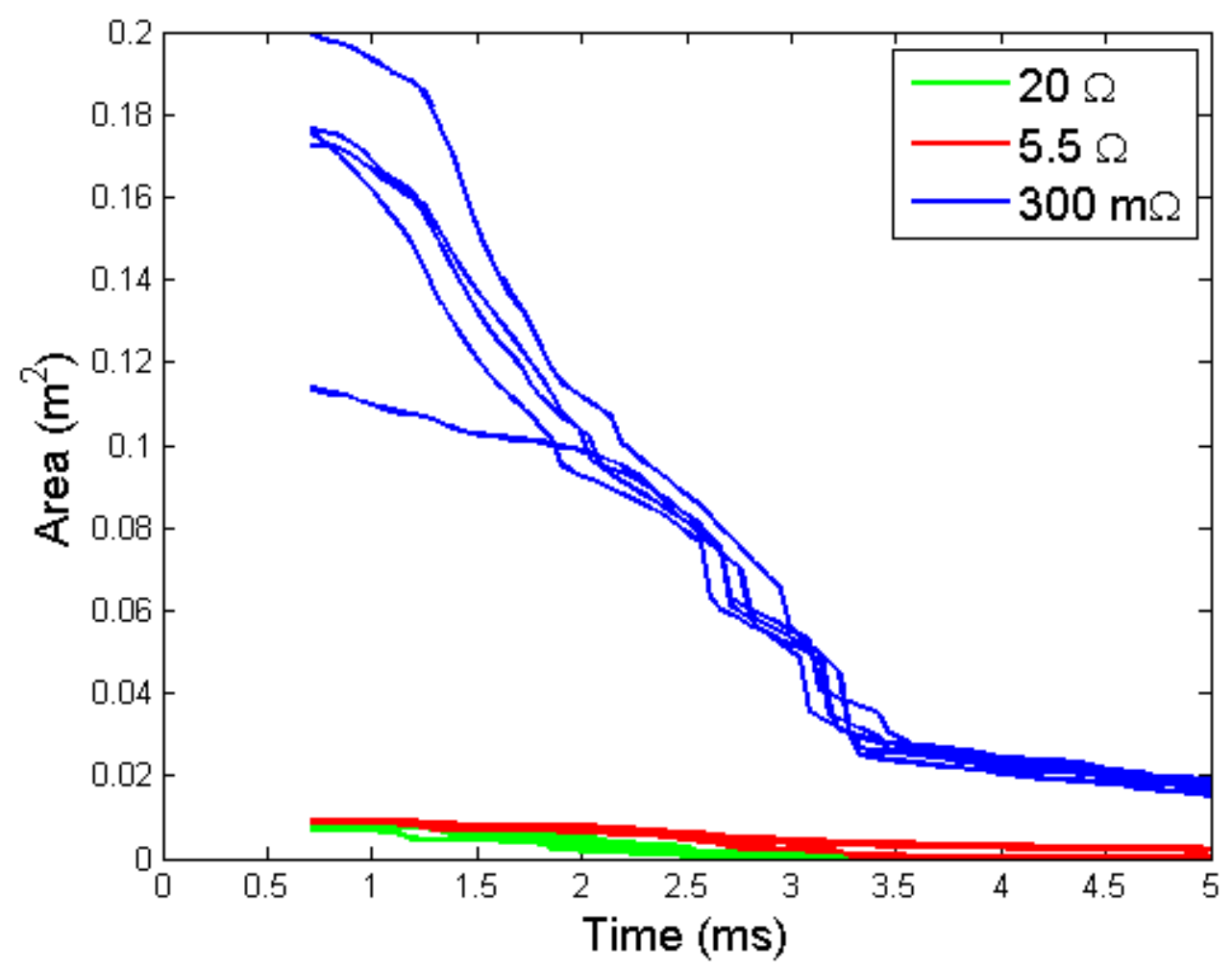

Figure 6.31 Area data of TAP discharges of varying circuit resistance

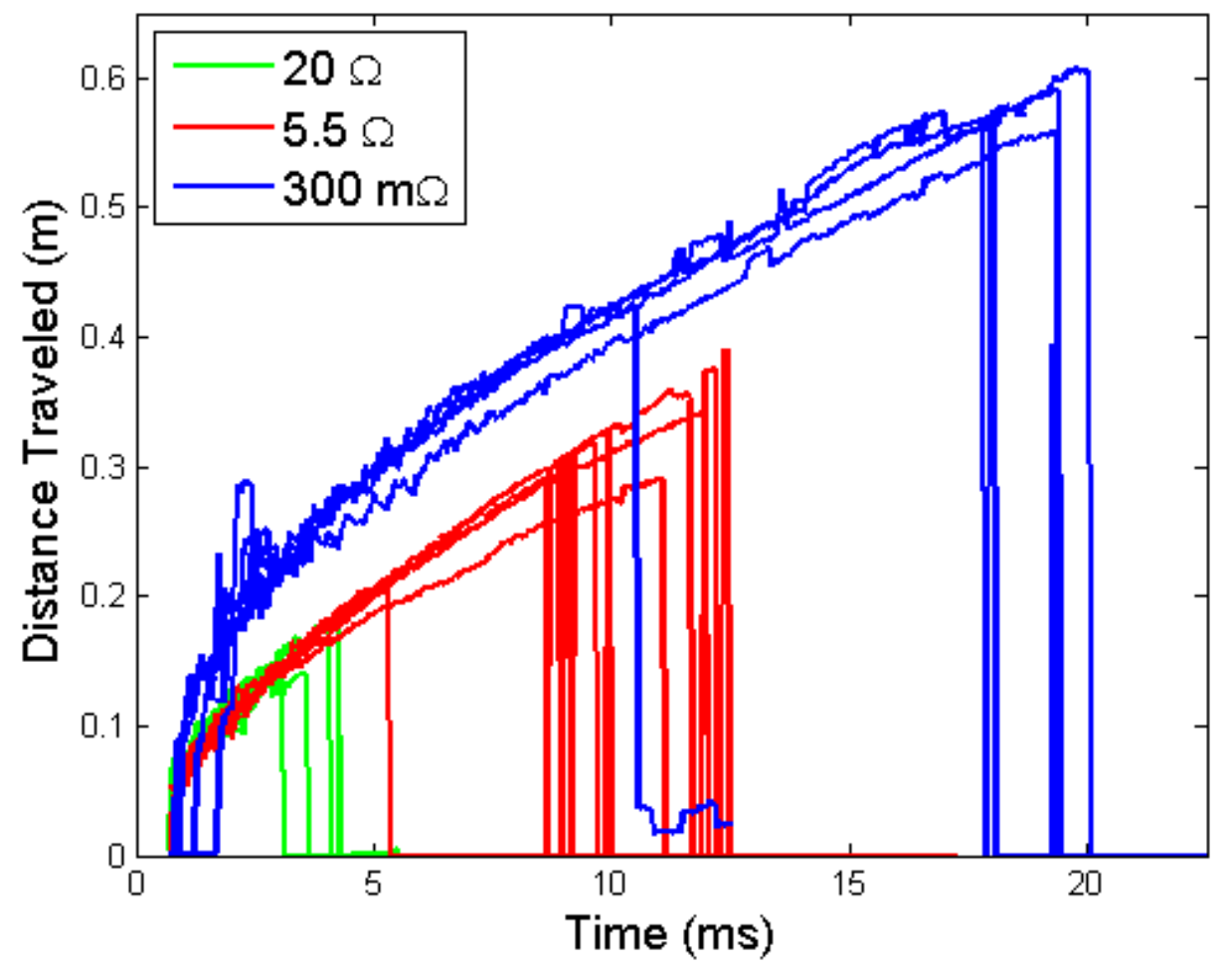

Figure 6.32 Distance traveled data of TAP discharges of varying circuit resistance 

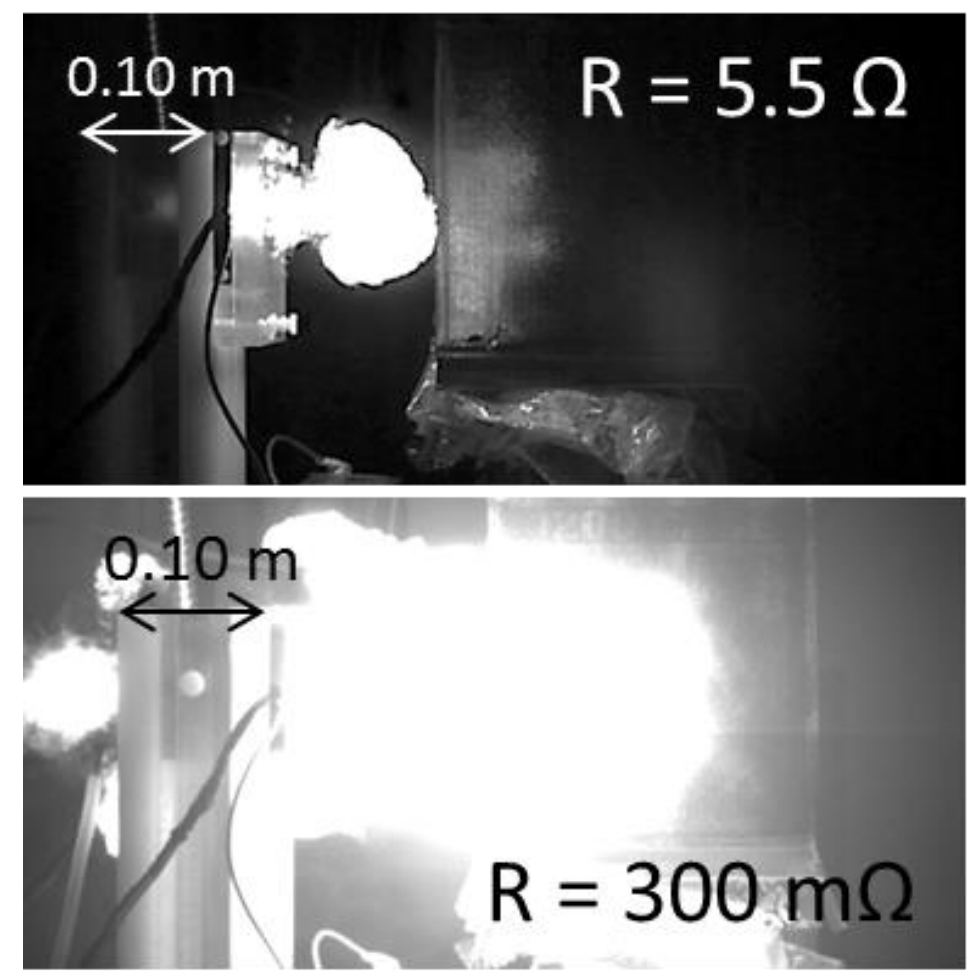

Figure 6.33 High speed images of the TAP $997 \mu$ from wire vaporization for two resistor configurations.

For a more visible comparison of the difference between the typical configuration $\left(\mathrm{R}_{1}=5.5 \Omega, \mathrm{L}_{1}=11.77 \mu \mathrm{H}\right)$ and the underdamped case $\left(\mathrm{R}_{1}=300 \mathrm{~m} \Omega, \mathrm{L}_{1}=11.77 \mu \mathrm{H}\right)$, Figure 6.33 shows high speed images of the TAP from the two conditions $997 \mu$ s after wire vaporization. These images show the dramatic difference in size and luminosity. On average, the underdamped trials produced discharges which were over ten times the size of discharges in the prototypical circuit configuration for the first 4 milliseconds. The underdamped condition produced discharges with $72 \%$ longer duration (up to $20 \mathrm{~ms}$ ) which traveled $76 \%$ farther (up to $0.60 \mathrm{~m}$ ). These are the largest TAP discharges yet observed.

It is clear that the circuit deposited more energy into the exploding wire as a result of removing the resistor in the circuit. Unfortunately, the resultant increase in size of the 
plasma discharge made it unsafe for the diagnostic probes to obtain accurate voltage measurements of the voltage through the exploding wire during these experiments. Thus, the energy deposited into the discharge from the plasma circuit was not calculated from measured results. The simulation program discussed in Section 3.1 and Appendix A has been used to simulate this condition. Changing the simulation parameters to reflect a $300 \mathrm{~m} \Omega$ circuit resistance, resulted in an energy deposited of approximately $727 \mathrm{~J}$. When compared to the other energy analysis previously presented this is over seven times larger than the typical simulated circuit. As discussed in Section 5.6.1, the simulation underestimates the energy deposited into the discharge, but an increased estimate is not possible without being able to measure the voltage and current through the exploding wire for this condition.

Ultimately, removing the aqueous-electrolyte resistor from the circuit created an underdamped circuit condition resulting in further energy to be discharged into the exploding wire. This additional energy significantly increased the volume and duration of the TAP discharge. This enhancement to the TAP cannot be overstated in its ability to increase the applicability of this discharge. This enhanced version of the TAP was large enough to raise safety concerns in the laboratory, thus experiments were limited in this program to the initial discovery and several attempts at using it in shock wave experiments (discussed in Chapter 8). Future experiments should focus on the redesign of some of the laboratory equipment and diagnostics to further characterize this enhanced TAP discharge. 


\subsection{Magnetic Quadropole Experiments}

To attempt to verify the duration of the TAP by means other than light emission, experiments were conducted with a magnetic fixture to alter the TAP's momentum. If the TAP is still a plasma in its later toroidal stages, it will consist of numerous charged particles and should exhibit acceleration due to the magnetic Lorentz force, Equation 2.9.

Four super magnets were configured in a quadrupole geometry to create a magnetic field pattern in the path of the TAP discharge. A plastic ring with a $0.133 \mathrm{~m}$ inner diameter, shown in the left image of Figure 6.34, was fabricated to hold the magnets while also allowing for the TAP to pass directly through the magnetic field. The fixture holds the magnets about $0.07 \mathrm{~m}$ from the axis of the ring and is placed $0.165 \mathrm{~m}$ from the front face of the plasma fixture. The right image of Figure 6.34 shows the view of the Phantom camera with the TAP discharge firing toward the magnetic fixture. In this image, at $2.470 \mathrm{~ms}$ from 'first light,' the TAP has not yet traveled close enough to the magnetic fixture for any change to occur.
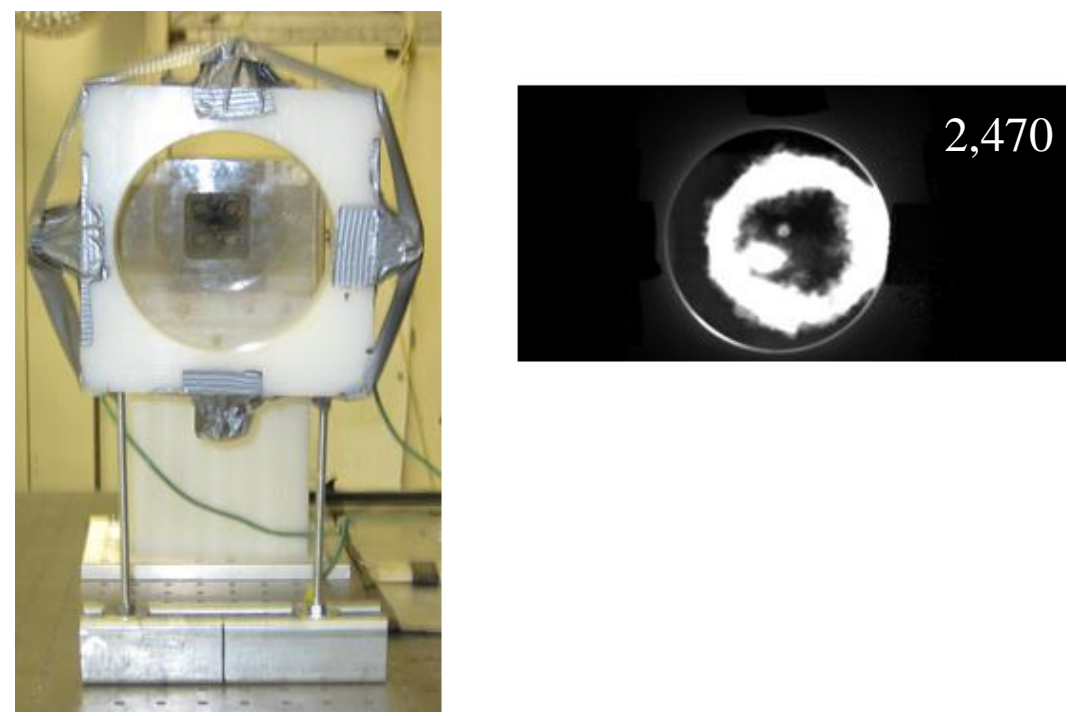

Figure 6.34 Magnetic quadropole fixture, with magnets. The inner ring diameter is approximately $0.133 \mathrm{~m}$ (left). The image on the right is the TAP discharge propagating toward the fixture. 
Figure 6.35 shows images from the Phantom v7.3 of the TAP passing through the magnetic quadropole. These images are $142.5 \mu$ s apart with a $2 \mu$ s exposure. The first image is $3.990 \mathrm{~ms}$ from the first light of the plasma. As shown, the toroid shape collapses toward the axis.
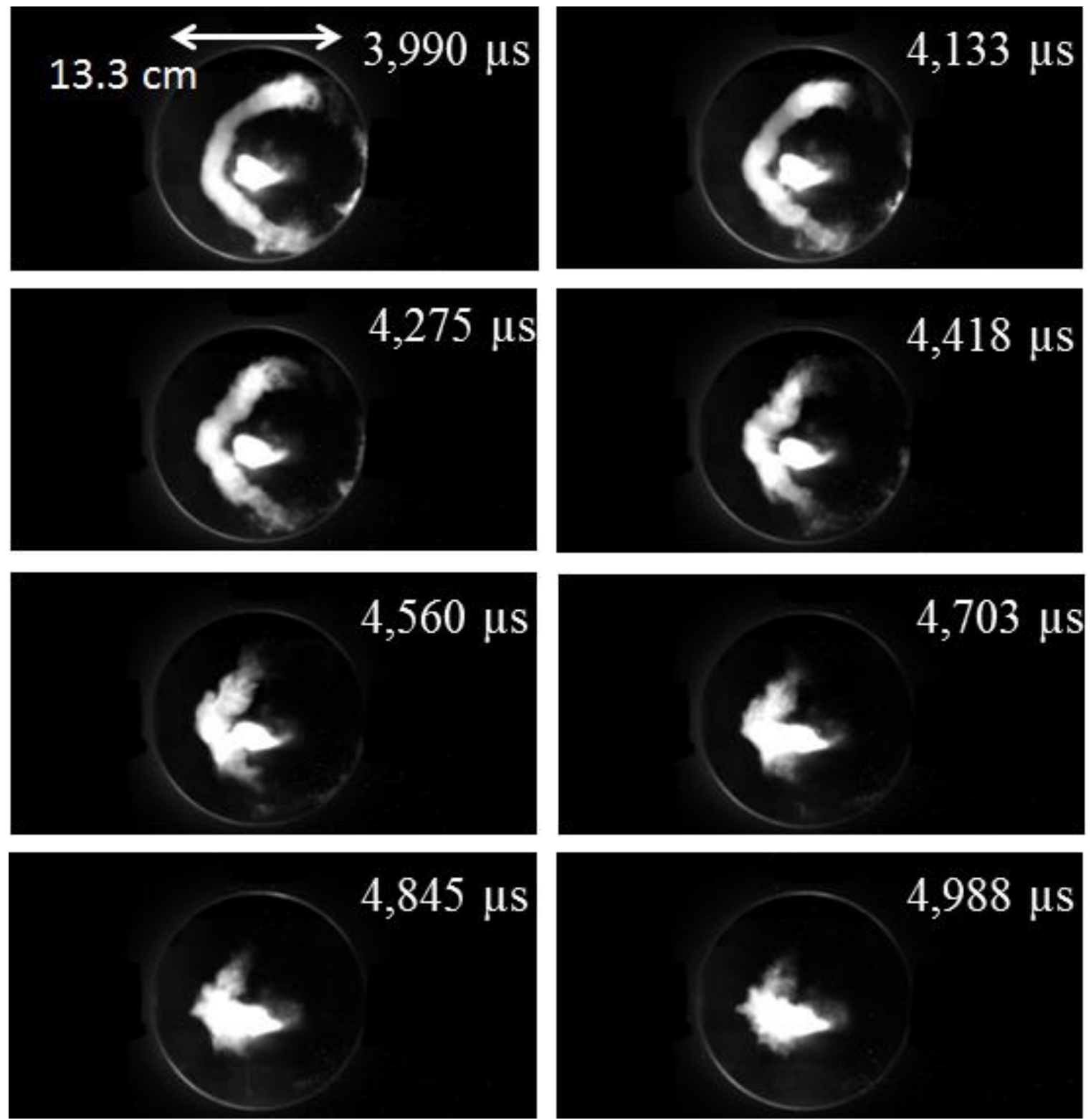

Figure 6.35 Images of the TAP passing through the magnetic quadropole (scaling and timing information are given in the images) 
Figure 6.36 shows images of the same event from the perspective of the SVSI high speed camera. For these experiments, the SVSI high speed camera filmed at a frame rate of 2,130 fps with a $469 \mu$ s interframe time and a $9 \mu$ s exposure. The image on the right in this figure is $0.939 \mathrm{~ms}$ after first light of the plasma. Subsequent images from the SVSI shown in Figure 6.37 depict the TAP traveling through the magnetic fixture, starting at $3.756 \mathrm{~ms}$ and collapsing over the next $2 \mathrm{~ms}$ as time advances.
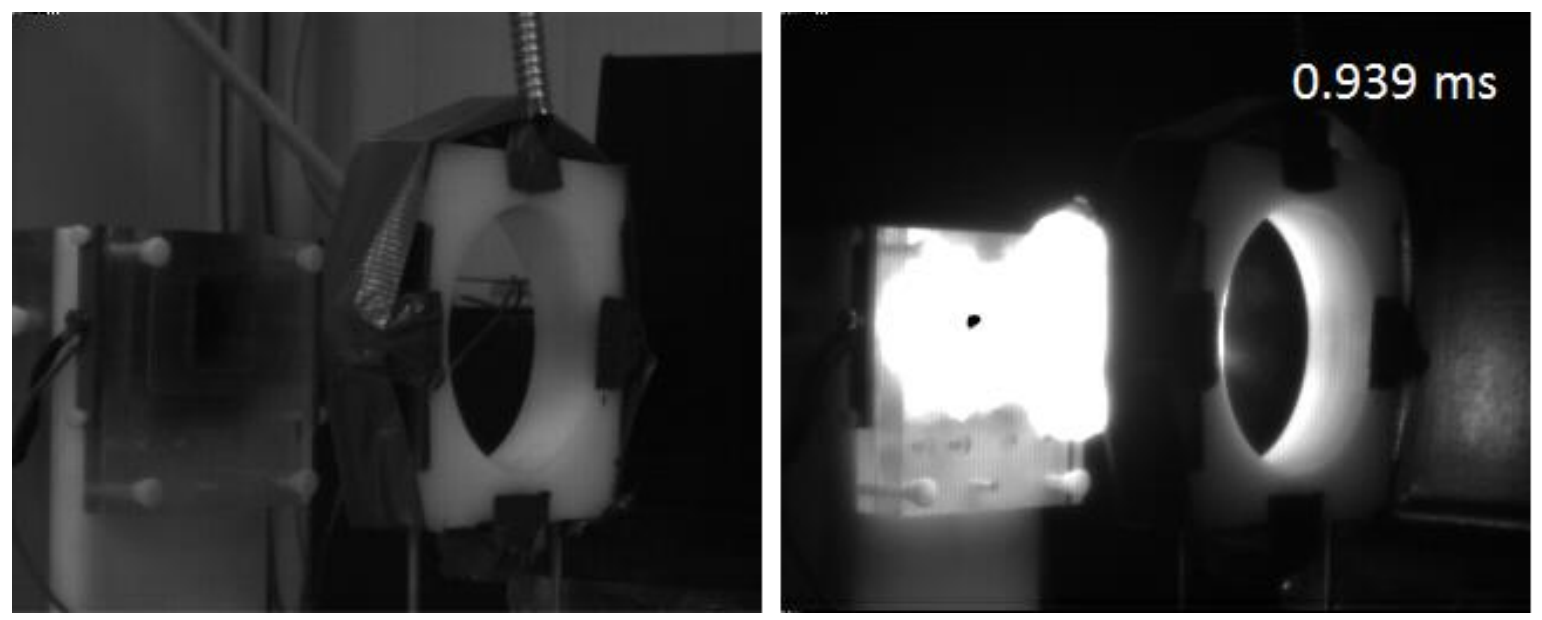

Figure 6.36 TAP expanding toward the magnetic fixture from the perspective of the SVSI

From Figure 6.36, and Figure 6.37 shown on the next page, it is apparent that the TAP has many charged particles several milliseconds after formation. The plasma is seen to collapse as it passes through the magnetic quadrupole, demonstrating Lorentz forces on the plasma. 

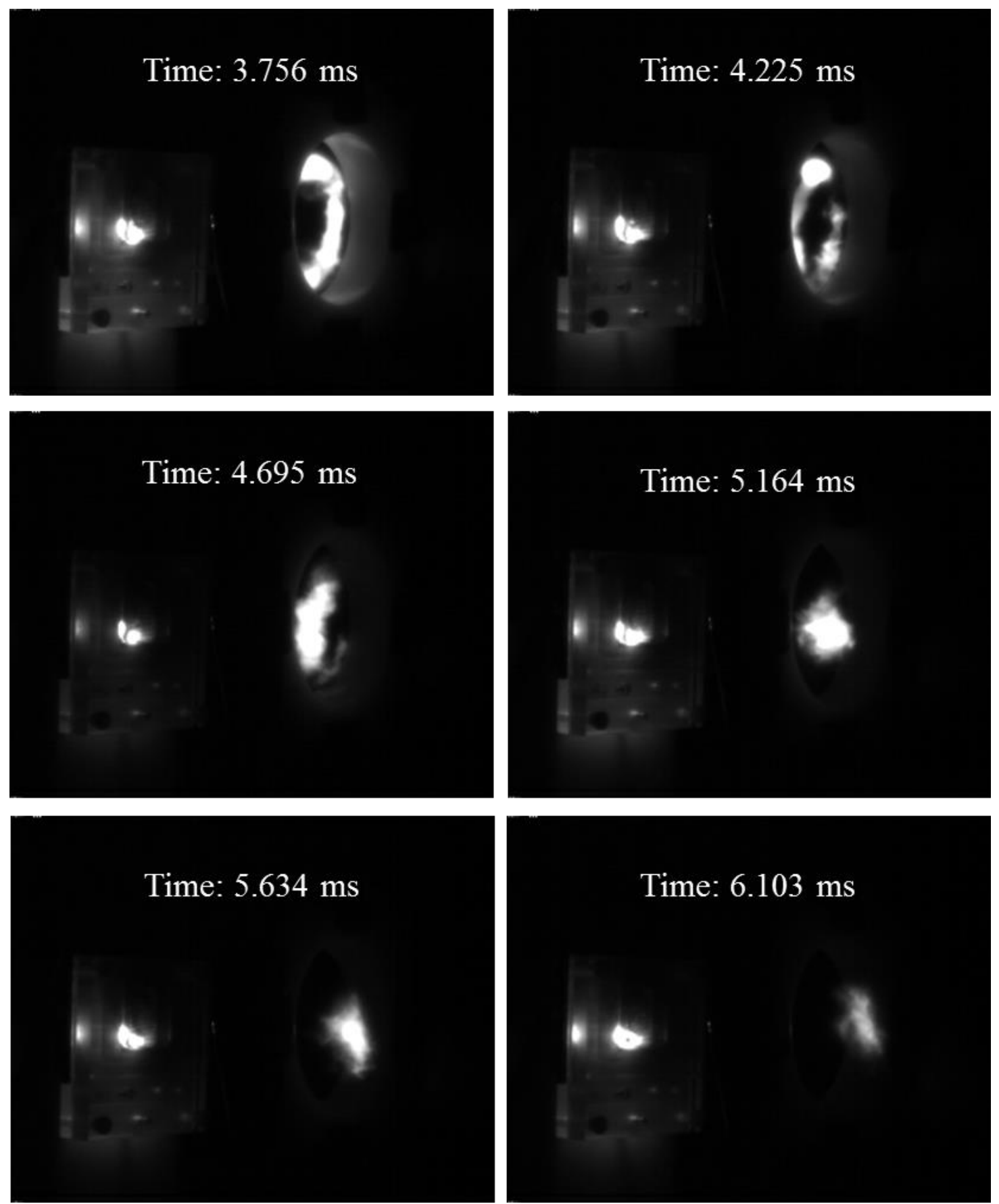

Figure 6.37 High speed images of the TAP passing through the magnetic quadropole 


\subsection{Measuring the Shock Wave from the Plasma-Generating Exploding Wire}

A series of experiments were conducted with the Kistler 603B1 pressure sensors to measure the pressure front from the exploding wire of the plasma circuit. The two sensors were shielded from RF signals and placed at various locations in front of the plasma fixture. Figure 6.38 depicts the initial placement of the sensors along with a coordinate system (dimensions are in meters). The sensors were placed at the following locations: $(0,0.15),(0.075,0.15),(0.15,0.15),(0.23,0.15),(0,0.305),(0.075,0.305),(0.15,0.305)$, $(0.23,0.305)$.

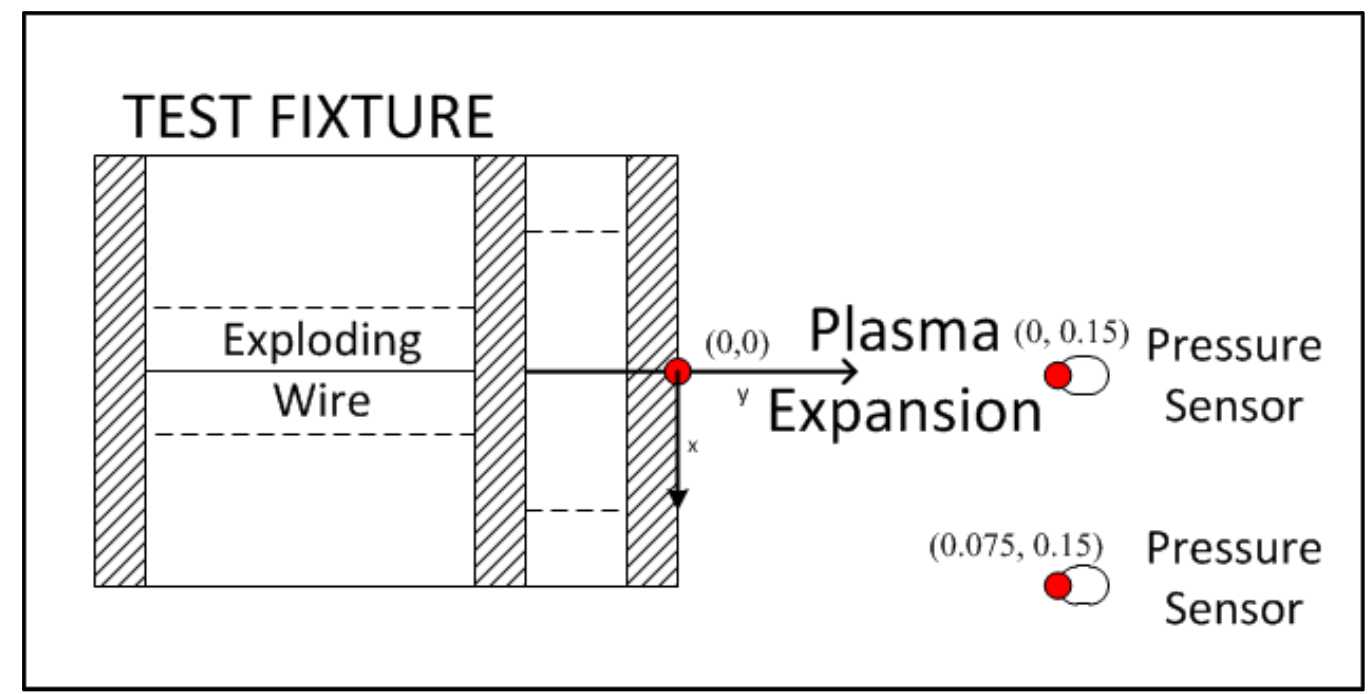

Figure 6.38 Diagram of pressure sensor placement for exploding wire shock wave measurements

The pressure wave fronts of these experiments are shown in Figure 6.39 and Figure 6.40 where the $\mathrm{x}$-axis is the time from vaporization of the exploding wire. A table of the peak amplitude and time of arrival is given for each experiment condition in Table 6.3. The magenta colored line labeled as the 'No Screen' condition was an experimental trial in which the accelerator screen was removed. As discussed in Section 5.5 this condition resulted in a smaller and shorter duration plasma that does not form a toroidal shape. 


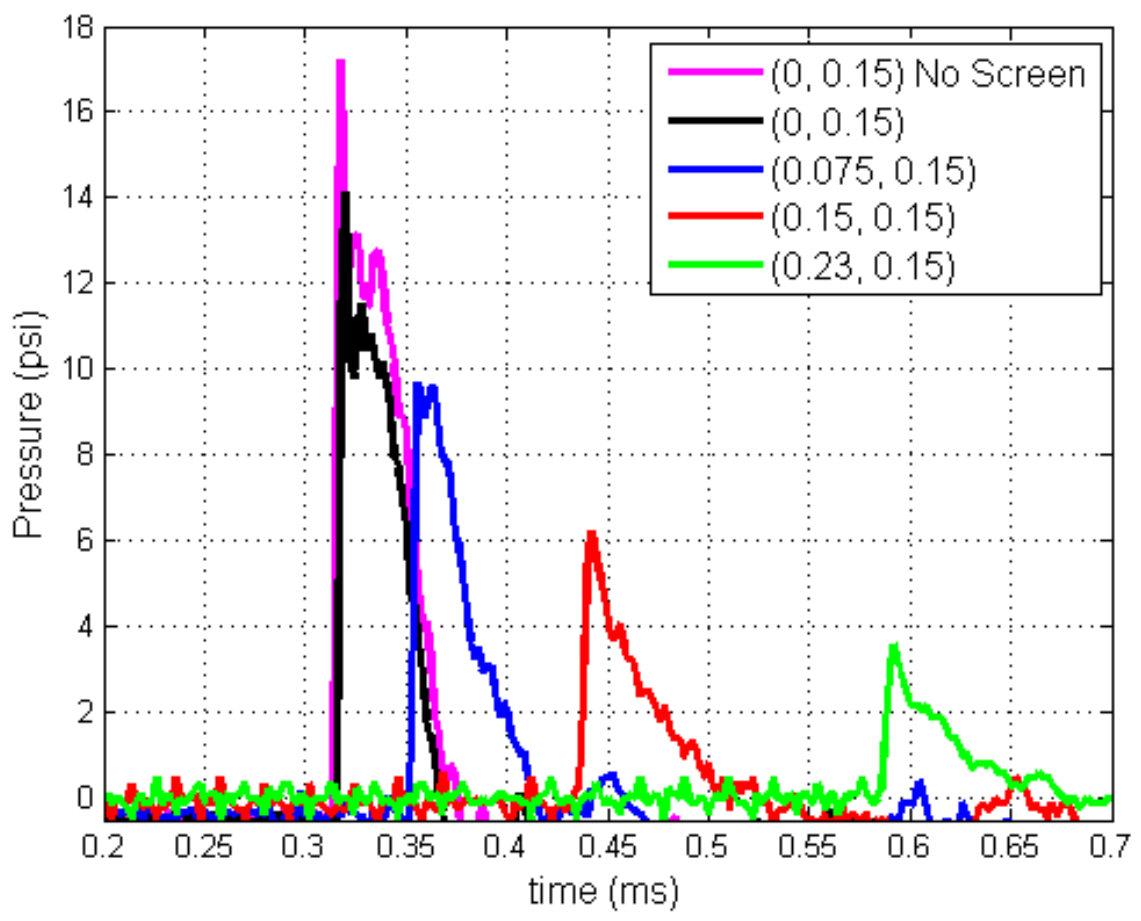

Figure 6.39 Pressure waveforms of the shock wave from the plasma-generating exploding wire. The legend describes the location of the pressure sensor (units are in $\mathrm{m}$, coordinate system is shown in Figure 6.38). More data shown in Figure 6.40.

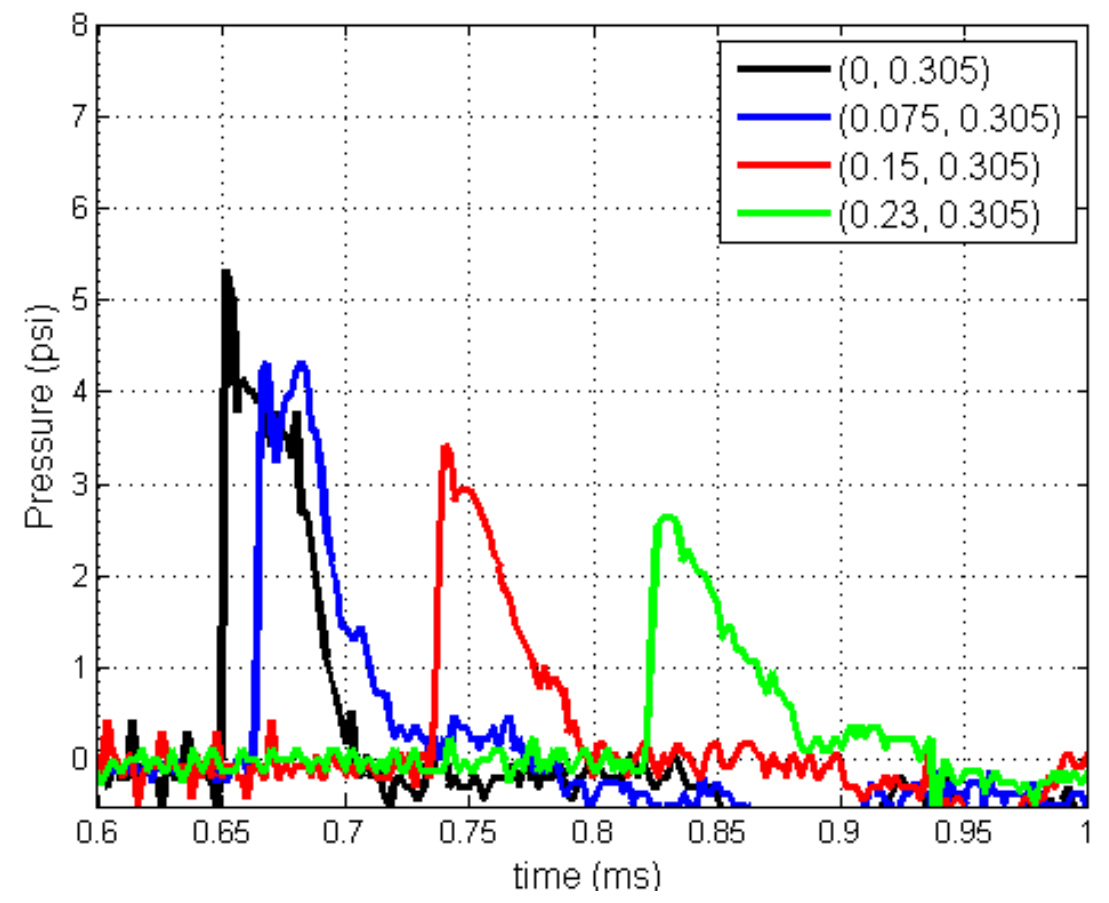

Figure 6.40 Pressure waveforms of the shock wave from the plasma-generating exploding wire (continued data from Figure 6.39). The legend describes the location of the pressure sensor (units are in $\mathrm{m}$, coordinate system shown in Figure 6.38). 
These results show a pressure front as high as $13 \mathrm{psi}$ at approximately $0.15 \mathrm{~m}$ from the plasma test fixture. This shock wave spread and decrease in amplitude is proportional to the square of the distance from the plasma fixture. The front has passed the region of interaction approximately $850 \mu \mathrm{s}$ after wire vaporization. This is at least $1.5 \mathrm{~ms}$ before the desired interaction time, and the primary shock wave has not yet exited the shock tube. Therefore, the pressure front from the exploding wire plasma is considered to not affect the interaction of the primary shock wave and the TAP discharge.

Table 6.1 Peak amplitude and time of arrival data of the pressure wave from the plasma circuit exploding wire. The shock wave amplitude decrease is proportional to the square of the distance from the plasma fixture.

\begin{tabular}{|r|r|r|r|}
\hline $\begin{array}{c}\text { Pressure Sensor } \\
\text { Placement }(\mathrm{m}) \\
\text { (*denotes no } \\
\text { accelerator } \\
\text { screen) }\end{array}$ & $\begin{array}{c}\text { Peak } \\
\text { Amplitude } \\
\text { (psi) }\end{array}$ & $\begin{array}{c}\text { Time } \\
\text { of } \\
\text { Arrival } \\
\text { (ms) }\end{array}$ \\
\hline $0^{*}$ & $0.15^{*}$ & 17.22 & 0.318 \\
\hline $0^{*}$ & $0.15^{*}$ & 17.58 & 0.314 \\
\hline 0 & 0.15 & 13.02 & 0.32 \\
\hline 0 & 0.15 & 13.02 & 0.322 \\
\hline 0.075 & 0.15 & 9.6 & 0.362 \\
\hline 0.075 & 0.15 & 9 & 0.354 \\
\hline 0.15 & 0.15 & 6.24 & 0.442 \\
\hline 0.15 & 0.15 & 6.48 & 0.452 \\
\hline 0.23 & 0.15 & 3.6 & 0.592 \\
\hline 0.23 & 0.15 & 3.72 & 0.576 \\
\hline 0 & 0.305 & 5.34 & 0.652 \\
\hline 0 & 0.305 & 5.46 & 0.66 \\
\hline 0.075 & 0.305 & 4.32 & 0.668 \\
\hline 0.075 & 0.305 & 4.56 & 0.674 \\
\hline 0.15 & 0.305 & 3.42 & 0.74 \\
\hline 0.15 & 0.305 & 3.42 & 0.742 \\
\hline 0.23 & 0.305 & 2.64 & 0.828 \\
\hline 0.23 & 0.305 & 3 & 0.826 \\
\hline
\end{tabular}




\section{References for Chapter 6}

1. A. Lodes and R.D. Curry, "Critical Circuit Parameters in Producing a Toroidal Air Plasma,” Power Modulator \& High Voltage Conf, pp. 562-565, 2012. 


\section{CHAPTER 7: SPECTROSCOPY MEASUREMENTS}

A useful diagnostic in observing and characterizing air plasmas is the measurement of the spectral emission. Observing and analyzing the spectral emission of a plasma can yield insights into the plasma temperature, plasma charge density, electron density, and composition of the plasma [1-4]. An optical multichannel analyzer was configured into a spectrographic system for the purpose of characterizing the air plasma discharge as described in Section 4.6. Spectral emission data of the TAP discharge is presented and analyzed in this chapter.

\subsection{Local Thermal Equilibrium (LTE)}

When describing a plasma system, local thermal equilibrium (LTE) occurs when neighboring groups of electrons and heavy particles (ions and atoms) within the plasma have the same temperature. In LTE, the ion density is described by the Saha-Boltzmann relation, given in Equation 7.1 [5].

$$
n_{S B}=[n(g)]^{+} n_{e} \frac{g(p)}{2[g(g)]^{+}}\left[\frac{h^{2}}{2 \pi m_{e} k T_{e}}\right]^{3 / 2} \exp \left[\frac{\chi(p)}{k T_{e}}\right]
$$

Here $n_{s b}(p)$ and $g(p)$ are the Saha-Boltzmann population density and the statistical weight respectively of level $p$. Also, $[n(g)]^{+}$and $[g(g)]^{+}$are the population density and the statistical weight of the ground state $g$ of the ion having charge greater than that of level $p$ by 1 , and $\chi(\mathrm{p})$ is the ionization potential of level $p$. In this equation $k$ is the Boltzmann's constant, $h$ is Planck's constant, $T_{e}$ is the temperature of the gas, $\mathrm{m}$ is the mass of the electron, and $n_{\mathrm{e}}$ is the electron density.

Local groups of particles in a LTE plasma are able to efficiently exchange energy over short mean free paths and collision times such that only small deviations from a Maxwellian distribution can occur $[1,6]$. A plasma in LTE can be characterized by a 
small number of parameters which may be deduced from measurements such as spectral intensities of the emitted radiation $[6,7]$. Similar research has been conducted to establish a list of criteria for when LTE is an appropriate assumption for different types of laboratory plasmas [5-8]. The spectroscopic measurements described in this chapter assume that the TAP discharge is in LTE.

The present TAP discharge is assumed to be in LTE for two major reasons. First, atmospheric pressure air plasmas, like the TAP, are often thought to be in local thermodynamic equilibrium due to the high number of interspecies collisions [8]. Second, the TAP discharge is a multi-millisecond duration discharge, and electron recombination, diffusion, and attachment are all present over this long duration [1-3, 6].

\subsection{Spectral Emission of the TAP}

Using the Acton Research Corporation SpectraPro-300i spectrograph system, the spectral emission profile of the TAP was measured [10]. The experiment was repeated over a four-window, 200-1000*10-9 $\mathrm{m}$ spectrum, consisting of five tests for each win...dow. The center wavelengths observed were $300 * 10^{-9} \mathrm{~m}, 500 * 10^{-9} \mathrm{~m}, 700 * 10^{-9} \mathrm{~m}$, and $900 * 10^{-9} \mathrm{~m}$. The CCD was gated over the first $5 \mathrm{~ms}$ of the emission lifetime of the TAP.

The center wavelength of the spectrograph was the only change of the experimental setup during these tests. All other settings with the system, other diagnostics, and the conditions of the circuit and test fixture were held constant throughout the experiments.

Figure 7.1 shows the corrected spectral emission of the TAP over the $200-800 \mathrm{~nm}$ range. Data from the $800-1000 * 10^{-9} \mathrm{~m}$ range were measured as minimal, and are not 
displayed. Spectral data in the range $200-400 * 10^{-9} \mathrm{~m}$ were corrected with the deuterium lamp. Data from the $400-1000 * 10^{-9} \mathrm{~m}$ range were corrected with the QTH lamp.

The data show that the most intense emission of the TAP is in the $350-400 * 10^{-9} \mathrm{~m}$ range. This would suggest the air plasma has a very strong oxygen II emission [11]. A peak also occurs near the nitrogen I lines at roughly $528 * 10^{-9} \mathrm{~m}$ [12]. Another local maximum can be seen near $650^{*} 10^{-9} \mathrm{~m}$, which would suggest a contribution by the hydrogen Balmer- $\alpha$ line an emission line used to study the electron density of the TAP later in this chapter [13].

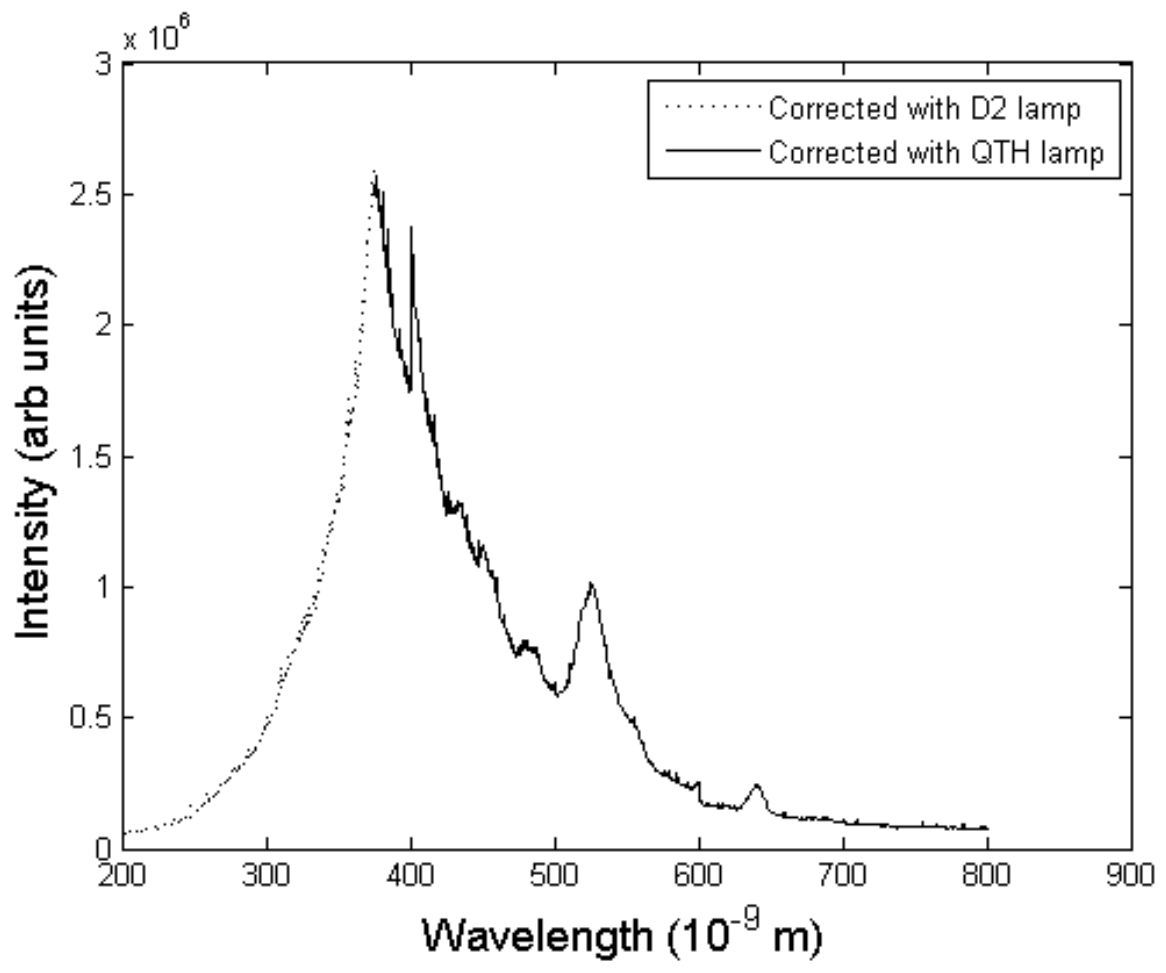

Figure 7.1 Corrected spectral emission of the TAP

This emission data can also be used to calculate a black body temperature of the TAP. By assuming LTE, the plasma can be modeled as a black body source [2, 3]. The radiation of a black body source is described by Planck's Law in Equation 7.2 [2, 3].

$$
I=\frac{2 h v^{3}}{c^{2}}\left[\exp \left(\frac{h v}{k T}\right)-1\right]^{-1}
$$


The intensity, I, of the radiation field, measured in $\left[\mathrm{W} \mathrm{m} \mathrm{mr}^{-1}\right]$, is frequency and temperature dependent $[2,3]$. The temperature of a black body radiation is related to its maximum photon flux at a certain wavelength, described in Wein's displacement law, Equation $7.3[2,3]$. The spectrum peaks at $375^{*} 10^{-9} \mathrm{~m}$ corresponding to a black body temperature of the TAP of 7,733 K using Equation 7.3 [10].

$$
\lambda_{\max }=\frac{2.9 * 10^{3}}{T}
$$

\subsection{Temperature Measurements of the TAP}

Additional experiments were conducted to measure the temperature of the TAP discharge. The spectroscopy system was thoroughly recalibrated to increase spectral resolution. The spectrometer was taken apart and cleaned, the fiber realigned and remounted, and the iCCD was refocused on the exit slit of the spectrometer. Then, Newport pencil calibration lamps model $6035(\mathrm{Hg}-\mathrm{Ar})$ and model $6032(\mathrm{Ne})$ were used to measure the optimal spectral resolution of the $1200 \mathrm{~g} / \mathrm{mm}$ grating of the system. The FWHM of emission lines from these calibration lamps were found to be $0.2-0.4^{*} 10^{-9} \mathrm{~m}$. This high resolution grating is center-blazed at $300 * 10^{-9} \mathrm{~m}$, restricting practical diagnostic application to wavelengths below $500 * 10^{-9} \mathrm{~m}$.

The spectral emission of the TAP discharge was observed in the spectral range of 362- $404 * 10^{-9} \mathrm{~m}$. This range was chosen for calibration purposes, as the $\mathrm{Hg}$ - $\mathrm{Ar}$ lamp has two strong emission lines in this region $\left(365.02 * 10^{-9} \mathrm{~m}\right.$ and $\left.404.66 * 10^{-9} \mathrm{~m}\right)$. The iCCD was gated for the initial $200 \mu$ s of the plasma discharge, and the data was calibrated after collection in MATLAB $®$ by using data collected from the Hg-Ar lamp and data from the QTH lamp. Five experimental trials were averaged, and the calibrated, averaged data is shown in Figure 7.2. 
The collected spectrum was then analyzed for identifiable atomic transition lines using the NIST atomic spectral database [14]. Many spectral features were identified as Fe I or Cr I transitions. As noted in Chapter 3, the high voltage cathode and the accelerator grid are both composed of stainless steel, which is primarily composed of iron and has components of chromium as well. These features are reasonable to expect, as the fiber is positioned to collect light approximately $0.02 \mathrm{~m}$ from the accelerator screen (the location of the TAP discharge during the initial $200 \mu \mathrm{s}$ ).

Eleven Fe I lines were chosen for an analysis commonly known as a Boltzmann plot which is used to obtain an estimate of the temperature of the iron ions $[15,16]$. Other features were identified in the collected spectrum, but were discarded because of close proximity to other Fe I lines (blending) or other likely radiation sources (chromium lines). The lines chosen are annotated by arrows in Figure 7.2 and the information associated with each atomic transition is given in Table 7.1 from the NIST atomic spectral database [14].

For a plasma in LTE, the populations of the energy levels for a particular species at a given position within the plasma are given by the Boltzmann equation, Equation 7.4,

$$
\frac{N_{j}}{N^{Z}}=\frac{g_{j} \exp \left(-\frac{E_{j}}{k T}\right)}{Q^{Z}(T)}
$$

where the superscript $z$ refers to the ionization of the species, $N_{j}, E_{j}$, and $g_{j}$ are the population $\left[\mathrm{m}^{-3}\right]$, energy $[\mathrm{eV}]$, and degeneracy (unitless) of the upper level of the species respectively, $\mathrm{N}^{\mathrm{z}}$ is the number density of the species $\left[\mathrm{m}^{-3}\right], \mathrm{k}$ is Boltzmann's constant $\left[\mathrm{e} \mathrm{K}^{-1}\right], \mathrm{T}$ is the electron temperature $[\mathrm{K}]$ and $\mathrm{Q}^{\mathrm{Z}}[\mathrm{T}]$ is the partition function of the species, which becomes a constant in the following analysis [1]. 


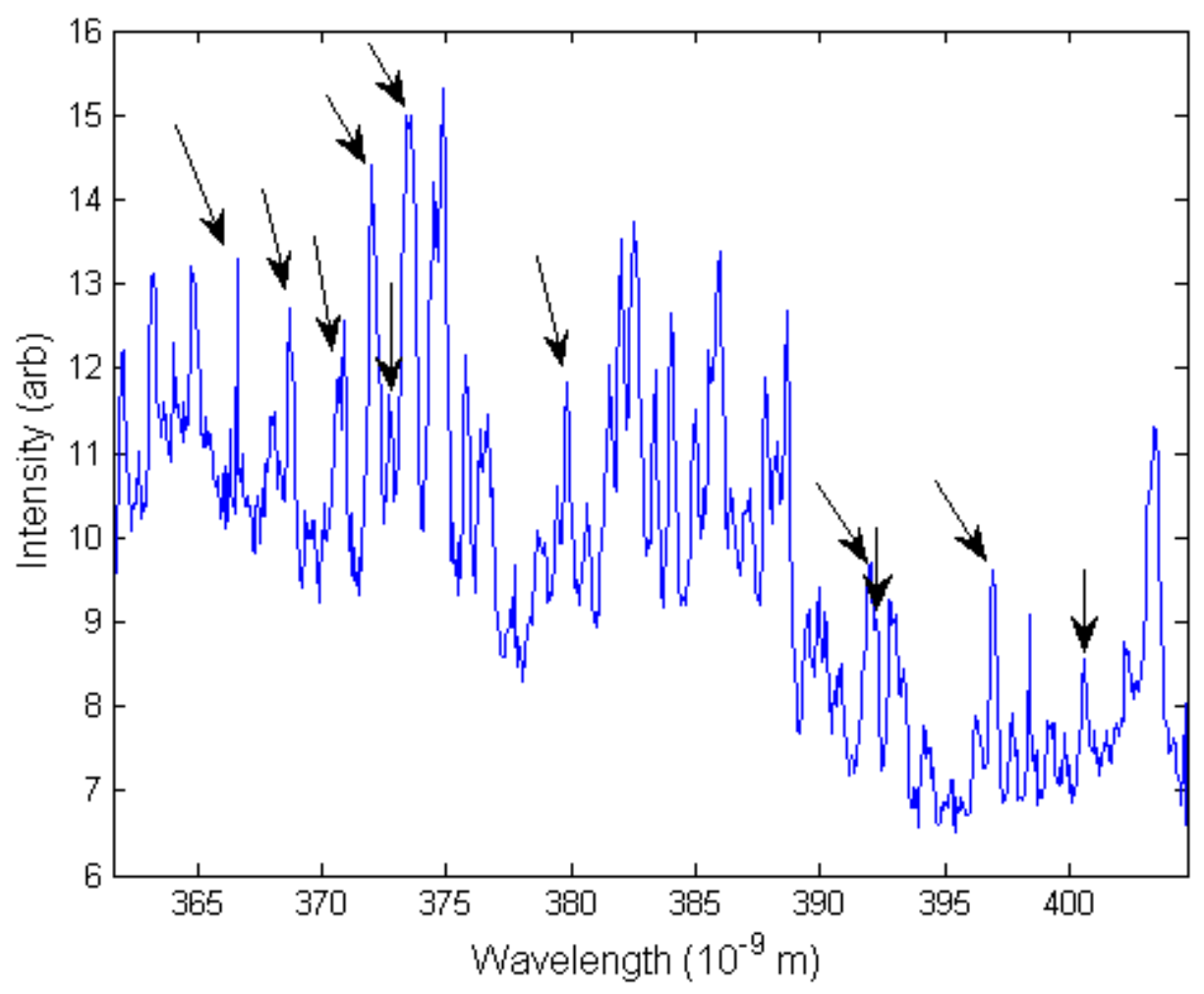

Figure 7.2 Calibrated average spectrum of the TAP for the initial 200 us after wire vaporization. The black arrows annotate Fe I lines chosen for Boltzmann plot analysis.

Table 7.1 Wavelength, transition probabilities, lower and upper energy levels, and upper level degeneracy for the Fe I emission lines used for Boltzmann analysis [14]

\begin{tabular}{|c|c|c|c|c|}
\hline Wavelength & $\begin{array}{c}\text { Transition } \\
\text { Probabilities }\end{array}$ & $\begin{array}{c}\text { Lower } \\
\text { Energy } \\
\text { Level }\end{array}$ & $\begin{array}{c}\text { Upper } \\
\text { Energy } \\
\text { Level }\end{array}$ & $\begin{array}{c}\text { Upper } \\
\text { Level } \\
\text { Degeneracy }\end{array}$ \\
\hline$\lambda_{\mathrm{ij}}$ & $\mathrm{A}_{\mathrm{ij}}$ & $\mathrm{E}_{\mathrm{i}}$ & $\mathrm{E}_{\mathrm{j}}$ & $\mathrm{g}_{\mathrm{j}}$ \\
\hline $\mathrm{nm}$ & $10^{\wedge} 8 \mathrm{~s}^{-1}$ & $\mathrm{eV}$ & $\mathrm{eV}$ & \\
\hline 367.99131 & $1.38 \mathrm{E}-02$ & 0.0000 & 3.3683 & 9 \\
\hline 368.74564 & $8.00 \mathrm{E}-02$ & 0.8590 & 4.2204 & 9 \\
\hline 370.55658 & $3.21 \mathrm{E}-02$ & 0.0516 & 3.3965 & 7 \\
\hline 372.25627 & $4.97 \mathrm{E}-02$ & 0.0873 & 3.4170 & 5 \\
\hline 372.76188 & $2.24 \mathrm{E}-01$ & 0.9582 & 4.2833 & 5 \\
\hline 373.33173 & $6.48 \mathrm{E}-02$ & 0.1101 & 3.4302 & 3 \\
\hline 379.95473 & $7.31 \mathrm{E}-02$ & 0.9582 & 4.2204 & 9 \\
\hline 392.02578 & $2.60 \mathrm{E}-02$ & 0.1213 & 3.2830 & 3 \\
\hline 392.29115 & $1.08 \mathrm{E}-02$ & 0.0516 & 3.2112 & 9 \\
\hline 396.9257 & $2.26 \mathrm{E}-01$ & 1.4849 & 4.6076 & 7 \\
\hline 400.52417 & $2.04 \mathrm{E}-01$ & 1.5574 & 4.6520 & 5 \\
\hline
\end{tabular}


By taking the natural logarithm of Equation 7.4, and simplifying terms, a linear equation can be written, given in Equation $7.5[15,16]$

$$
\ln \left(\frac{I_{\lambda} \lambda_{i j}}{A_{i j} g_{j}}\right)=-\left(\frac{1}{k T}\right) E_{j}+C
$$

where $I_{\lambda}$ is the measured intensity at the wavelength of transition, $\lambda_{i j}$ is the wavelength of the transition $(\mathrm{nm}), \mathrm{A}_{\mathrm{ij}}$ is the transition probability $\left(10^{8} \mathrm{~s}^{-1}\right)$, and $\mathrm{C}$ is a constant. Given a set of intensity data, a linear plot (the so called Boltzmann Plot) can be generated and the temperature of the plasma may then be deduced from the slope $[15,16]$.

This analysis was conducted on the eleven Fe I transitions observed in the TAP discharge, and a plot of the results is given in Figure 7.3. The eleven data points were fit to a line corresponding to a plasma temperature of $6,127 \pm 885 \mathrm{~K}$. The linear fit was very reasonable with an $\mathrm{R}^{2}$ value of 0.9503 , suggesting a strong fit to Boltzmann distribution within the plasma during this time period. This plasma temperature is also within $20 \%$ of the black body temperature calculated in the previous section.

Again, this analysis is an approximate analysis to obtain the plasma temperature because we are under the assumption the plasma is in LTE in this location and time. In this case, the energy state of the Fe ions is being used to calculate the temperature of those ions, which is equivalent to the plasma temperature assuming a Boltzmann distribution and LTE. 


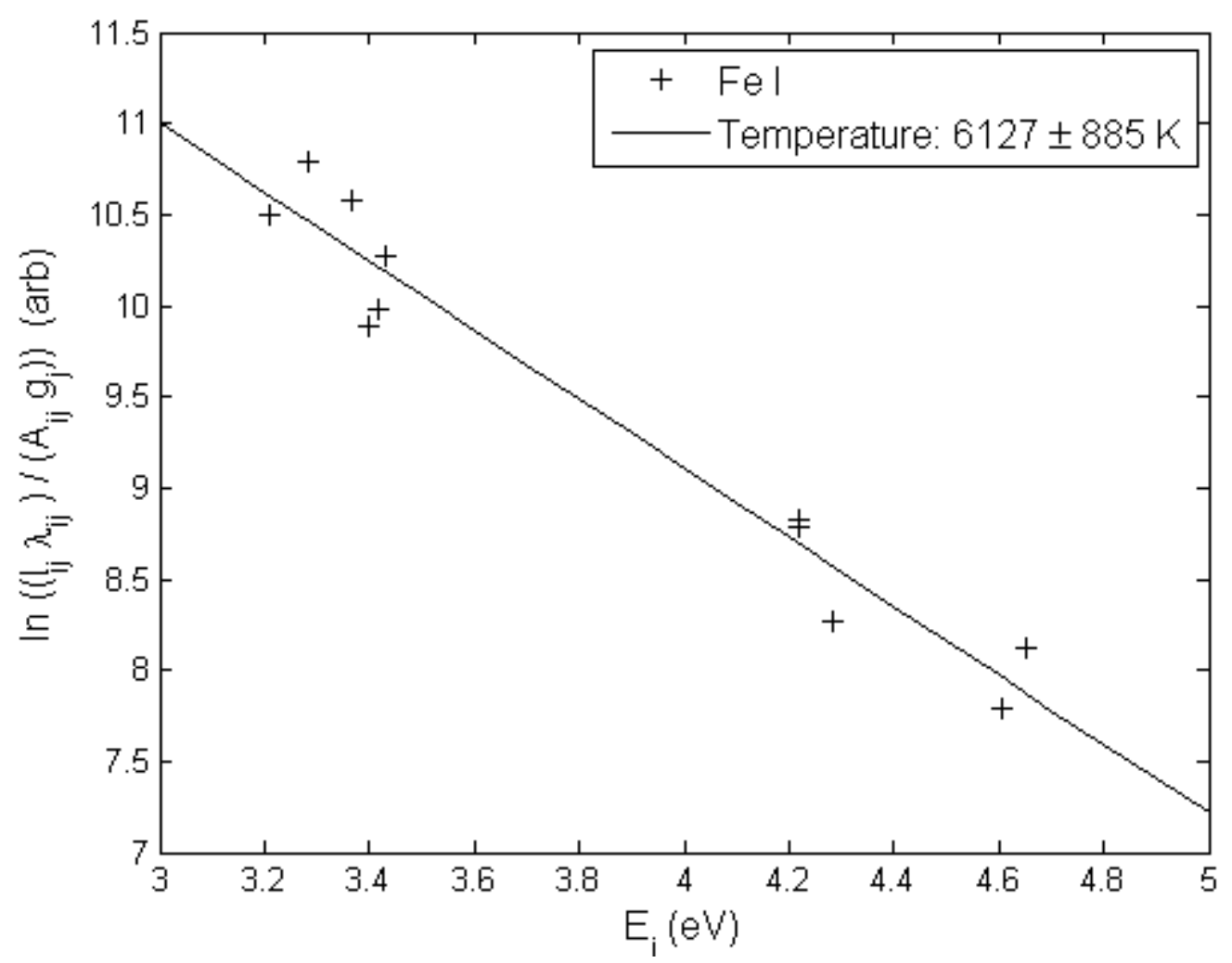

Figure 7.3 Boltzmann plot of intensity measurements of Fe I transition lines from the TAP discharge. The linear fit corresponds to a plasma temperature of $6127 \pm 885 \mathrm{~K}$.

\subsection{Stark Broadening Measurements of the TAP}

As discussed in Chapter 2, the mitigation effect that a plasma has on a shock wave is dependent on the electron density of the discharge. As such, great efforts have been made to estimate and characterize the electron density of the plasma. Initially, broadening measurements were explored, which is why the spectrometer was calibrated as mentioned in the previous section.

The Stark effect is the pressure broadening of spectral lines by Coulomb forces from charged particles [1 - 4]. This broadening is typically very strong in plasmas. As a diagnostic, Stark broadening can be a means to estimate the electric field present inside a plasma, which is proportional to the charged particle density by an analysis heavily rooted in quantum mechanics and perturbation theory. Hydrogen lines are often studied 
for this diagnostic as the shifts by the particle-produced fields are large and thus an approximation method, known as the quasistatic approximation, is valid [1 - 4]. From this analysis, the electron density $n_{\mathrm{e}}$, in $\mathrm{m}^{-3}$, can be approximated from the halfwidth, in $\mathrm{nm}$, of the hydrogen Balmer-alpha line $\mathrm{H}-\alpha$ at $656.28 \mathrm{~nm}$ by Equation 7.6 [4].

$$
\Delta \lambda_{\frac{1}{2}} \approx 2.8 * 10^{-17} * n_{e}^{0.72}
$$

After several experiments and analysis of the emission profile of the TAP near $\mathrm{H}-\alpha$ at $656.28 * 10^{-9} \mathrm{~m}$, it was determined that the spectroscopy system could not detect observable emission in this spectrum. The high resolution grating chosen for the system $\left(1.2^{*} 10^{6}\right.$ grooves $\left./ \mathrm{m}\right)$ is center-blazed at $300 * 10^{-9} \mathrm{~m}$, thus as the system is moved to observe the spectrum near $H-\alpha$, the grating's efficiency is very small. While $H-\alpha$ should be an emitter in the TAP discharge because it is an air plasma, hydrogen is not a very common element in air and hydrogen transitions are not very intense emitters (especially when compared to Fe I transitions). Several attempts were made to try to increase the hydrogen content of the discharge by humidifying the air immediately surrounding the test fixture, but no emission lines were successfully observed. It is likely that the spectroscopy system, when using the high resolution grating, is not able to observe the low intensity levels of the $\mathrm{H}-\alpha$ transitions. Section 7.2 observed a local maximum near $\mathrm{H}-$ $\alpha$ using the low resolution $3.0^{*} 10^{5}$ grooves/m grating which is center blazed at $500 * 10^{-9} \mathrm{~m}$. These previous measurements would suggest that a different high-resolution grating might be able to obtain a Stark broadening measurement of $\mathrm{H}-\alpha$ and obtain an estimate of the electron density. Due to the limited resources of this research program, this measurement is left to future investigations. 
Other commonly used Stark broadening transition lines were also explored. The H- $\beta$ and $\mathrm{H}-\gamma$ transition lines at $486.1 * 10^{-9} \mathrm{~m}$ and $434.1 * 10^{-9} \mathrm{~m}$ respectively were also not observable by the system. In future work, a replacement grating would greatly improve the capabilities of the system, possibly allowing these transition lines to be easily observed.

One transition line that could possibly be observed by the current system is the Fe I transition line at $538.34 * 10^{-9} \mathrm{~m}$. This line is commonly used for Stark broadening measurements and has a well-known Stark width coefficient $[15,17,18]$. This Fe I transition line might be observable by the system due its relatively high intensity of emission. However, the system cannot be calibrated in that spectrum with either of the Newport calibration pen lamps currently available. It should be possible to calibrate the system with Newport Xenon lamp 6033, if this measurement were pursued in the future.

\subsection{Saha-Eggert Calculations of the Electron Density}

With stark broadening measurements difficult to obtain, the electron density of the TAP was estimated by using the relative intensities of neutral atomic emission lines (Fe I) and singly charged ionic lines (Fe II). This relationship is known as the Saha-Eggert Equation and is given in Equation $7.7[15,16,19,20]$

$$
n_{e}=4.3 * 10^{15} \frac{I_{\lambda}^{0} g_{j}^{+} A_{i j}^{+} \lambda_{i j}^{0}}{I_{\lambda}^{+} g_{j}^{0} A_{i j}^{0} \lambda_{i j}^{+}} T^{3 / 2} \exp \left[\frac{-\left(V^{+}+E_{j}^{+}-E_{j}^{0}-\Delta V^{+}\right)}{k T}\right]
$$

where the superscripts $(0,+)$ represent the neutral atom and singly charged ion respectively, $\mathrm{V}^{+}$represents the ionization potential of the lower ionization stage $(7.9 \mathrm{eV}$ for $\mathrm{Fe}$ ), and $\Delta \mathrm{V}^{+}$is a correction to the ionization potential due to plasma interactions that can be neglected for the first ionization stage (in higher stages it must be used) [19]. 
For this analysis, the spectral emission of the TAP discharge was observed in the spectral range of $254-297 * 10^{-9} \mathrm{~m}$. This spectrum included many Fe II emission lines for the analysis and also includes two $\mathrm{Hg}$ emission lines for calibration $\left(253.65^{*} 10^{-9} \mathrm{~m}\right.$ and $\left.596.73 * 10^{-9} \mathrm{~m}\right)$. The iCCD was gated for the initial $200 \mu$ s of the plasma discharge, and the data was calibrated after collection in MATLAB ${ }^{\circledR}$ by using data collected from the Hg-Ar lamp and data from the Dueterium lamp, as discussed in Section 5.6.1. The calibrated spectrum is shown in Figure 7.4.

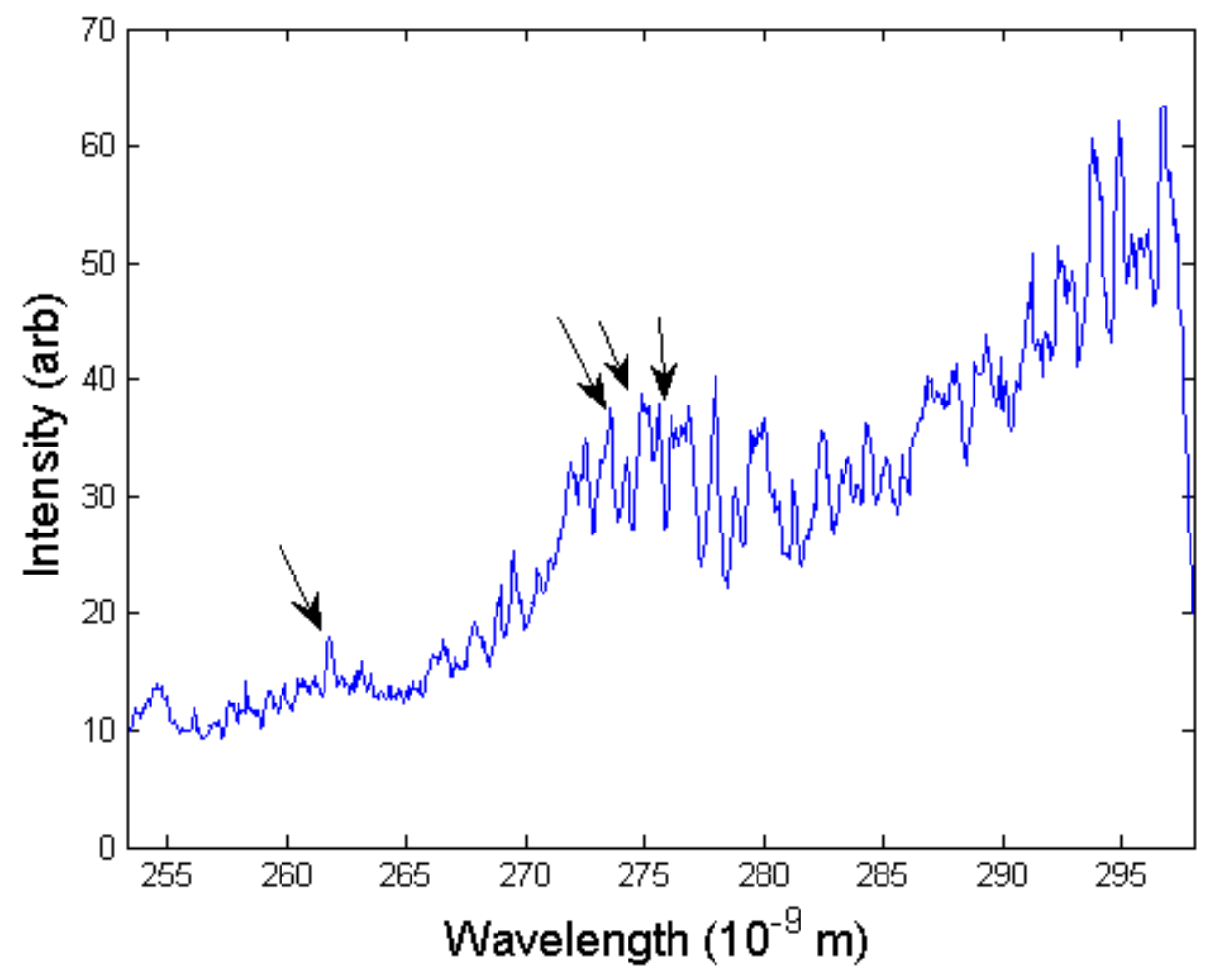

Figure 7.4 Calibrated spectrum of the TAP for the initial 200 us after wire vaporization. The black arrows annotate Fe II lines chosen for Saha-Eggert analysis 
Table 7.2 Wavelength, transition probabilities, lower and upper energy levels, and upper level degeneracy for the Fe II emission lines used for the Saha-Eggert Equation [14]

\begin{tabular}{|c|c|c|c|c|}
\hline Wavelength & $\begin{array}{c}\text { Transition } \\
\text { Probabilities }\end{array}$ & $\begin{array}{c}\text { Lower } \\
\text { Energy } \\
\text { Level }\end{array}$ & $\begin{array}{c}\text { Upper } \\
\text { Energy } \\
\text { Level }\end{array}$ & $\begin{array}{c}\text { Upper } \\
\text { Level } \\
\text { Degeneracy }\end{array}$ \\
\hline$\lambda_{\mathrm{ij}}$ & $\mathrm{A}_{\mathrm{ij}}$ & $\mathrm{E}_{\mathrm{i}}$ & $\mathrm{E}_{\mathrm{j}}$ & $\mathrm{g}_{\mathrm{j}}$ \\
\hline $\mathrm{nm}$ & $10^{\wedge} 8 \mathrm{~s}^{-1}$ & $\mathrm{eV}$ & $\mathrm{eV}$ & \\
\hline 261.7617 & $4.88 \mathrm{E}-01$ & 0.08278 & 4.8179 & 6 \\
\hline 273.9548 & $2.21 \mathrm{E} 00$ & 0.09863 & 5.51071 & 8 \\
\hline 274.9321 & $2.26 \mathrm{E} 00$ & 1.04047 & 5.54876 & 8 \\
\hline 275.5737 & $2.15 \mathrm{E} 00$ & .98633 & 5.48413 & 10 \\
\hline
\end{tabular}

The collected spectrum was then analyzed for identifiable atomic transition lines using the NIST atomic spectral database [14]. Many spectral features were identified as Fe I or Fe II transitions. Four Fe II transition lines, given in Table 7.2, were chosen for the Saha-Eggert analysis. Each Fe II transition was analyzed along with each Fe I transition line (the same eleven used in the Boltzman plot analysis of Table 7.1). These 44 combinations produced the calculated electron densities shown in Table 7.3, ranging from a minimum of $3.57 * 10^{14} \mathrm{~cm}^{-3}\left(3.57 * 10^{20} \mathrm{~m}^{-3}\right)$ to a maximum of $1.22 * 10^{15} \mathrm{~cm}^{-3}$ $\left(1.22 * 10^{21} \mathrm{~m}^{-3}\right)$ with an average of $7.36 * 10^{14} \mathrm{~cm}^{-3}\left(7.36 * 10^{20} \mathrm{~m}^{-3}\right)$.

This analysis is only meant as an estimate of the electron density as there are many inaccuracies that effect this calculation. The efficiency of the spectroscopy system in the ultraviolet spectrum degrades as the observed wavelength is decreased, as the fiber used for collection decreases in efficiency. The relatively low intensity of Fe II lines could also be a source of inaccuracy, as any nearby Fe I transition lines may be saturating the measurement. The average electron density of $10^{14} \mathrm{~cm}^{-3}\left(10^{20} \mathrm{~m}^{-3}\right)$ calculated is considered a good order of magnitude estimation for the TAP discharge. 
Table 7.3 Electron density $\left(\mathrm{cm}^{-3}\right)$ calculations of the 44 combinations of Fe I and Fe II emission lines using the Saha-Eggert Equation and the measured intensity of the TAP

\begin{tabular}{|c|c|c|c|c|c|}
\hline & & \multicolumn{4}{|c|}{ Calculated Electron Density $\left(\mathrm{m}^{-3}\right)$} \\
\hline & & \multicolumn{4}{|c|}{ Fe II Transition Lines } \\
\hline & $\lambda(\mathrm{nm})$ & 261.762 & 273.955 & 274.932 & 275.574 \\
\hline \multirow{11}{*}{$\begin{array}{c}\text { Fe I } \\
\text { Transition } \\
\text { Lines }\end{array}$} & 367.991 & $1.15 \mathrm{E}+21$ & $8.64 \mathrm{E}+20$ & $6.80 \mathrm{E}+20$ & $1.08 \mathrm{E}+21$ \\
\hline & 368.746 & $9.58 \mathrm{E}+20$ & $7.17 \mathrm{E}+20$ & $5.64 \mathrm{E}+20$ & $9.00 \mathrm{E}+20$ \\
\hline & 370.557 & $6.06 \mathrm{E}+20$ & $4.54 \mathrm{E}+20$ & $3.57 \mathrm{E}+20$ & $5.69 \mathrm{E}+20$ \\
\hline & 372.256 & $6.94 \mathrm{E}+20$ & $5.19 \mathrm{E}+20$ & $4.09 E+20$ & $6.52 \mathrm{E}+20$ \\
\hline & 372.762 & $6.45 \mathrm{E}+20$ & $4.83 \mathrm{E}+20$ & $3.80 \mathrm{E}+20$ & $6.06 \mathrm{E}+20$ \\
\hline & 373.332 & $9.48 \mathrm{E}+20$ & $7.10 \mathrm{E}+20$ & $5.59 \mathrm{E}+20$ & $8.91 E+20$ \\
\hline & 379.955 & $1.01 \mathrm{E}+20$ & $7.53 \mathrm{E}+20$ & $5.93 \mathrm{E}+20$ & $9.45 \mathrm{E}+20$ \\
\hline & 392.026 & $1.22 \mathrm{E}+21$ & $9.10 \mathrm{E}+20$ & $7.16 \mathrm{E}+20$ & $1.14 \mathrm{E}+21$ \\
\hline & 392.291 & $7.91 \mathrm{E}+20$ & $5.92 \mathrm{E}+20$ & $4.66 \mathrm{E}+20$ & $7.43 \mathrm{E}+20$ \\
\hline & 396.926 & $7.38 \mathrm{E}+20$ & $5.52 \mathrm{E}+20$ & $4.35 \mathrm{E}+20$ & $6.93 \mathrm{E}+20$ \\
\hline & 400.524 & $1.12 \mathrm{E}+21$ & $8.39 \mathrm{E}+20$ & $6.60 \mathrm{E}+20$ & $1.05 \mathrm{E}+21$ \\
\hline
\end{tabular}

Simulations in Section 3.1 predicted an electron density of $10^{18} \mathrm{~cm}^{-3}\left(10^{24} \mathrm{~m}^{-3}\right)$ for a fully ionized exploding wire discharge. This Saha-Eggert analysis suggests that the discharge is not fully ionized. Calculated estimates of the electron discharge were given in Section 5.6.3 which utilized the observed current and voltage waveforms. The electron density of the exploding wire discharge was estimated as $10^{16} \mathrm{~cm}^{-3}\left(10^{22} \mathrm{~m}^{-3}\right)$ from the pulsed power circuit diagnostics during wire vaporization and $10^{14} \mathrm{~cm}^{-3}\left(10^{20} \mathrm{~m}^{-3}\right)$ from the accelerator circuit diagnostics when the plasma has expanded between the accelerator screen and the high voltage cathode. The collection fiber was placed approximately $0.02 \mathrm{~m}$ beyond the accelerator screen, thus the estimated $10^{14} \mathrm{~cm}^{-3}\left(10^{20} \mathrm{~m}^{-3}\right)$ electron density calculated from the Saha-Eggert analysis agrees well with the calculations from the accelerator circuit diagnostics. 


\section{References for Chapter 7}

1. H. Griem, Plasma Spectroscopy, New York: McGraw Hill, 1964. pp. 29-31, 63-81, 107, 288-290.

2. T. Fujimoto, Plasma Spectroscopy, Oxford, New York: Oxford University Press, 2004, pp. 25-2, 205-212,213-235,236-256.

3. I. H. Hutchinson, Principles of Plasma Diagnostics, Cambridge, UK: Cambridge University Press, pp. 245-252.

4. H. J. Kunze, Introduction to Plasma Spectroscopy, Heidelberg, Germany: Springer 2009, pp. 155-166, 195-197.

5. T. Fujimoto and R. W. P. McWhirter, "Validity Criteria for Local Thermodynamic Equilibrium in Plasma Spectroscopy," Physical Review A, vol. 42, no. 11, 1990.

6. H. R. Griem, "Validitity of Local Thermal Equilibrium in Plasma Spectroscopy," Physical Reivew, vol. 131, no. 3, 1963.

7. K.T.A.L. Burm, "Deviations from Thermal Equilibrium in Plasmas," Phys. Lett. A, vol. 328, pp. 489-492, 2004

8. C. O. Laux, et al., "Optical Diagnostics of Atmospheric Pressure Air Plasmas," Plasmas Sources Sci \& Tech., vol. 12, pp. 125-138, 2003.

9. C. O. Laux, "Optical Diagnostics and Radiative Emision of Air Plasmas," PhD. Dissertation, Dept. Mech. Engr., Stanford University, 1993.

10. A. Lodes, et al., "Spectroscopic Measurements of a Toroidal Air Plasma" Pulsed Power Conf.,pp.1277-1281, 2011.

11. C. E. Moore, Tables of Spectra of Hydrogen, Carbon, Nitrogen, and Oxygen, J. W. Gallagher, ed., CRC Press, Inc., Boca Raton, FL, 1993.

12. C. E. Moore, "Selected Tables of Atomic Spectra, Atomic Energy Levels and Multiplet Tables - N I, N II, N III", Nat. Stand. Ref. Data Ser., NSRDS-NBS 3 (Sect. 5), pp. 80, 1975.

13. P. Zhao, et al., "Remeasurement of the Rydberg Constant," Physical Review A, vol. 34, no. 6, 1986.

14. NIST Atomic Spectra Database, http://physics.nist.gov

15. J.A. Aguilera and C. Aragon, "Characterization of a Laser-Induced Plasma by Spatially Resolved Spectroscopy of Neutral Atom and Ion Emissions. Comparison of Local and Spatially Integrated Measurements," Spectrochemica Acta Part B, vol. 59, pp 1861-1876, 2004.

16. B. Li and H. Li, "Discussion on Emission Spectroscopy Measurements from a Dense Electrothermal Launcher Plasma," Int. Symp. Ballistics, Interlaken, Switzerland, pp. 203-210, 2001.

17. J.A. Aguilera, et al., "Spatial Characterization of Laser-Induced Plasmas by Deconvolution of Spatially Resolved Spectra," J. Appl. Optics, vol. 42, no. 30, pp. 5938-5946, 2003.

18. C. Aragon and J.A. Aguilera, "Determind of the Local Electron Number Density in Laser-Induced Plasmas by Stark-Broadened Profiles of Spectral Lines, Comparitive results from $\mathrm{H \alpha}$, Fe I, and Si II lines," Spectrochemica Acta Part B, vol. 65, pp. 395-400, 2010. 
19. J. S. Cowpe, "Optical Emission and Mass Spectrometric Diagnostics of LaserInduced Plasmas," PhD Dissertation, School of Computing, Science and Engineering, University of Salford, UK, 2008.

20. S.H. Nam and Y. J. Kim, "Excitation Temperature and Electron Number Density Measured for End-On-View Inductively Coupled Plasma Discharge," Bull. Korean Chem. Soc., vol. 22, no. 8, pp. 827-832. 2001. 


\section{CHAPTER 8: INTERACTION EXPERIMENTS}

A block diagram of the interaction experiments is shown in Figure 8.1. High speed imaging and Schlieren imaging were used to observe the interaction and verify proper temporal and spatial interaction occurred between the shock wave and the TAP. These high speed videos yielded interesting qualitative results including changes in velocity, size, shape and duration of the TAP discharge as a result of interaction with a shock wave.

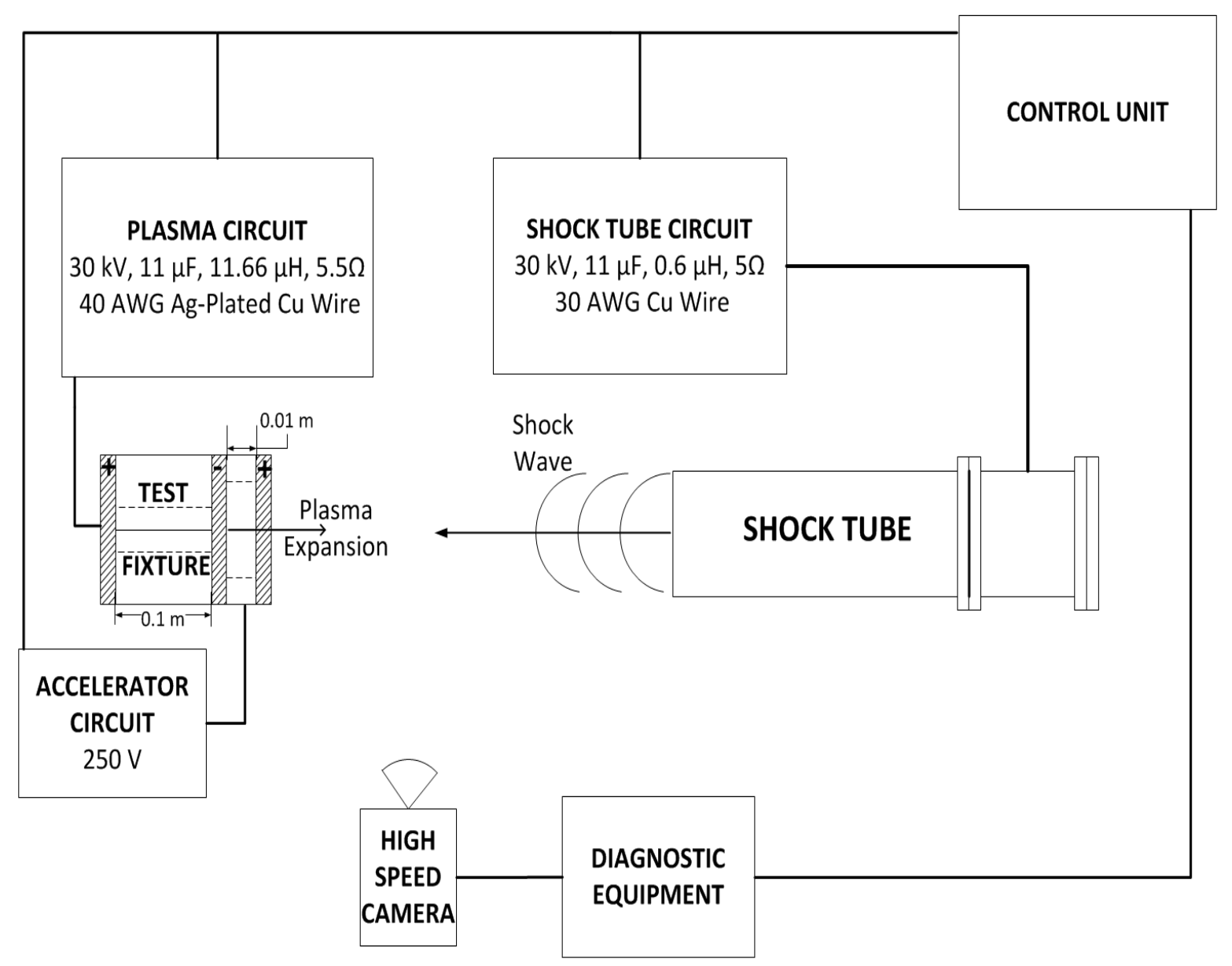

Figure 8.1 Experimental test bench 


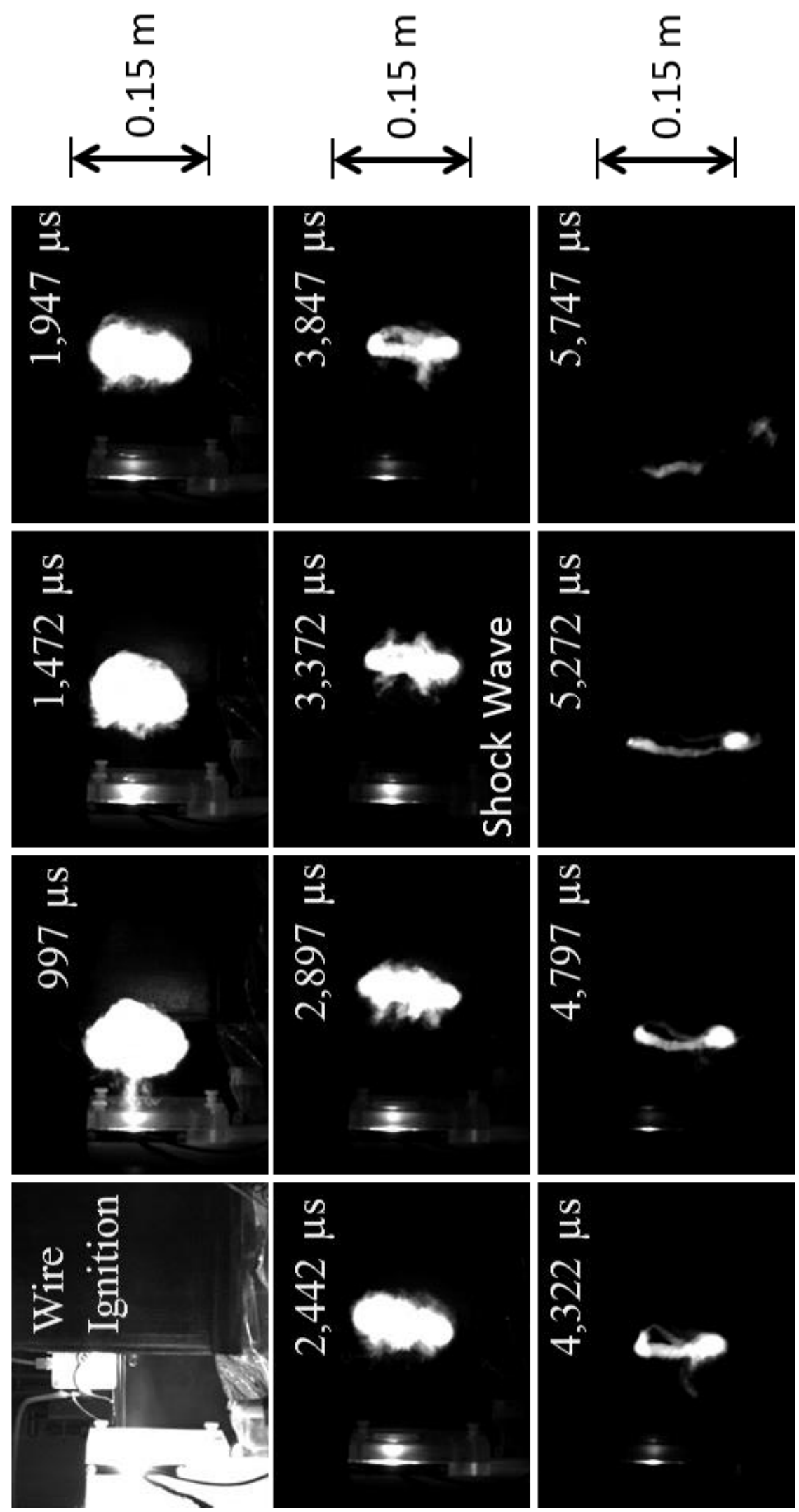

Figure 8.2 High speed images of a shock wave interacting with the TAP 
Figure 8.2 on the previous page, is a sequence of images from a high speed video taken with the Phantom v7.3. The selected images are labeled with the elapsed time after first light when the exploding wire is first ignited. For the first $2.5 \mathrm{~ms}$ after wire vaporization, the plasma discharge evolves, detaching from the fixture and propagating toward the shock tube. The shock front reaches the TAP at about $2.5 \mathrm{~ms}$ from first light, halting the TAP's propagation and slightly destabilizing the detached plasma discharge. As the TAP begins to form its toroid shape, the shock wave and subsequent reflections carry the TAP back toward the plasma fixture, reducing the plasma's size.

Figure 8.3 is a series of Schlieren images where the shock front travels through the TAP. The first image shows the shock front just before it collides with the plasma. The following images are $450 \mu \mathrm{s}, 900 \mu \mathrm{s}$, and $1,125 \mu \mathrm{s}$ later in time, respectively. These images show the TAP traveling back toward the plasma fixture while decreasing in size. Similar results have been published in [1].

Additional interaction experiments were conducted with the addition of the three Endevco $8530 \mathrm{C}-15$ pressure sensors. The three sensors were used to quantitatively characterize the effects the plasma had on the pressure front of the shock wave. These sensors were mounted behind the interaction region downstream from the shock wave to measure the pressure at the front plane of the plasma fixture. Each sensor was mounted $6 \mathrm{~cm}$ from the center axis of the exploding wire. One sensor was mounted toward the camera (Channel 2), one was mounted away from the camera (Channel 4), and one was mounted directly below the axis of the wire (Channel 3). 

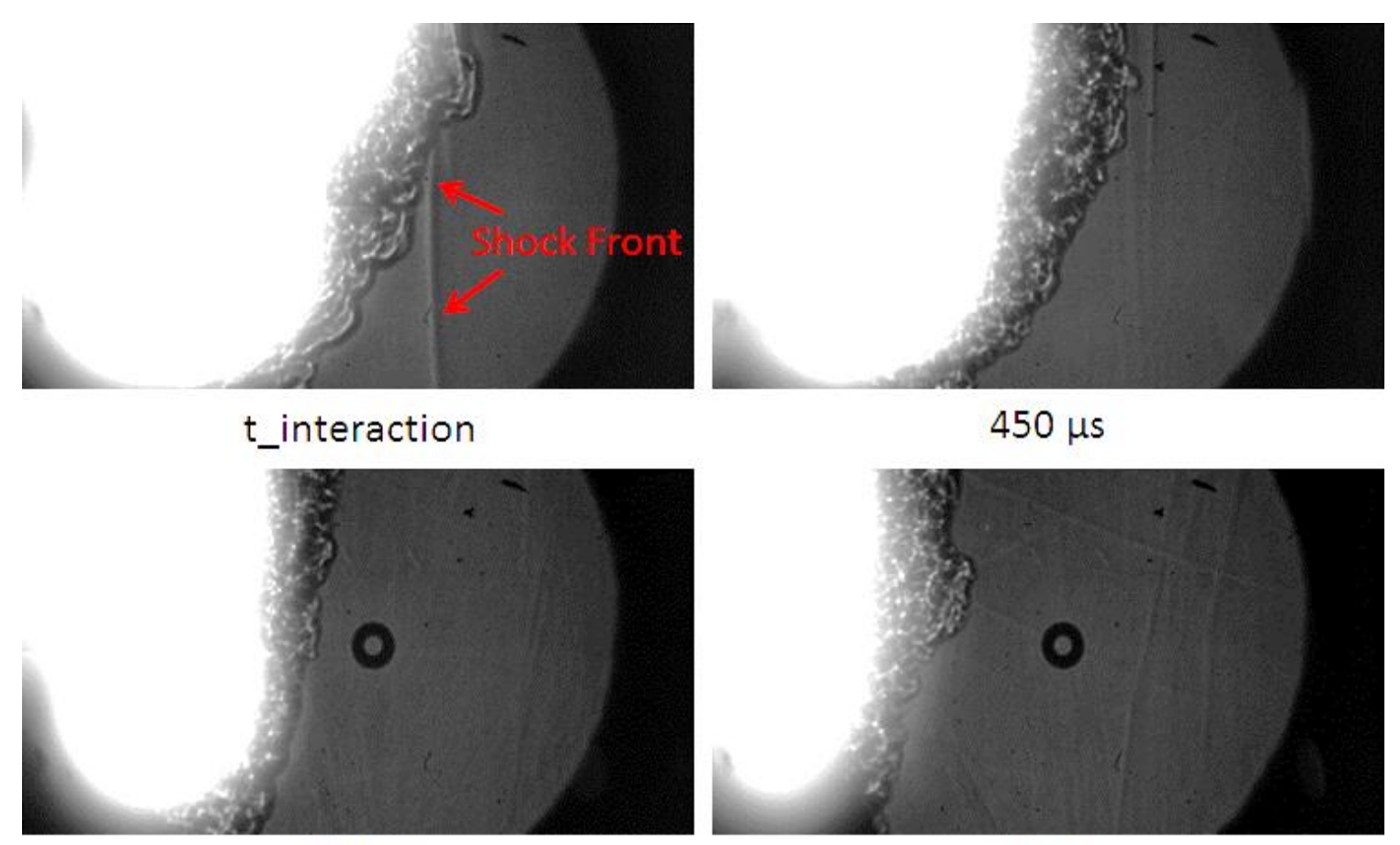

$900 \mu \mathrm{s}$

$1125 \mu \mathrm{s}$

Figure 8.3 Schlieren images of a shock wave interacting with the TAP. The shock wave is annotated with a red arrow in the first image. As time evolves beyond interaction, the TAP reverses velocity and decreases in size.

As discussed in Section 4.5, the Endevco pressure sensors are calibrated for a pressure range of $0-15$ psi, but are sensitive to the RF noise of the pulsed power circuits. Within the first exploratory trials, one of the pressure sensors (Channel 2) was damaged and was no longer responsive during subsequent experiments. This damage may have been a result of electrostatic discharge into the sensor or from a collection of particulates on the sensor's diaphragm deposited from the experiments, or directly from the shock wave. The resource constraints of this research program did not allow for the sensor to be repaired for use in further experimentation.

Pressure data collected from the other two pressure sensors showed inconsistent results. Several interaction experiments were conducted under the same experimental conditions, and the results were compared with experiments in which no plasma was discharged (shock wave only). Sample pressure waveforms are shown in Figure 8.4 and 
Figure 8.5. The waveforms in thick blue lines are the shock front from an experiment without a plasma interaction. The red waveforms are data from interaction experiments. Figure 8.5 is a selection of the data in Figure 8.4, to more easily compare the pressure fronts. Discarding noise effects, the TAP discharge resulted in a $28.8 \%$ reduction in peak amplitude from $8.32 \mathrm{psi}$ in the shock wave only data to $5.92 \mathrm{psi}$ in the interaction data.

MATLAB ${ }^{\circledR}$ was used to analyze all of the data from these experiments. A smoothing algorithm was utilized to eliminate the RF noise in the signals, and the data was corrected for the variations in the shock wave. The details of this analysis are given in Appendix E. The waveforms in Figure 8.4 and Figure 8.5 are shown unprocessed.

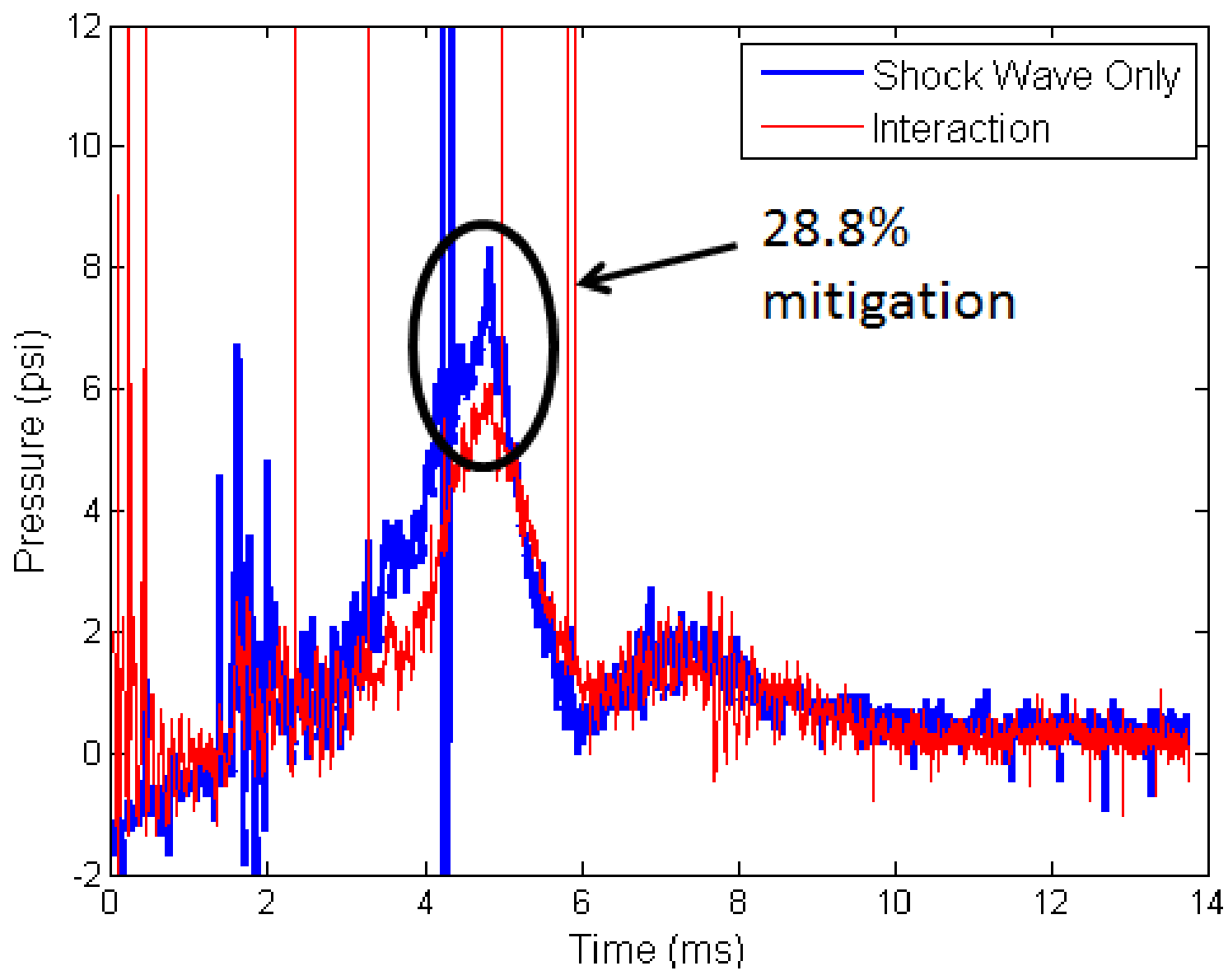

Figure 8.4 Example unprocessed pressure waveforms. This graph compares an unmitigated shock wave (blue) with a shock wave which has interacted with a TAP discharge (red). A $28.8 \%$ reduction in peak amplitude of the shock wave was observed. An expanded view of this data is given on the next page. 


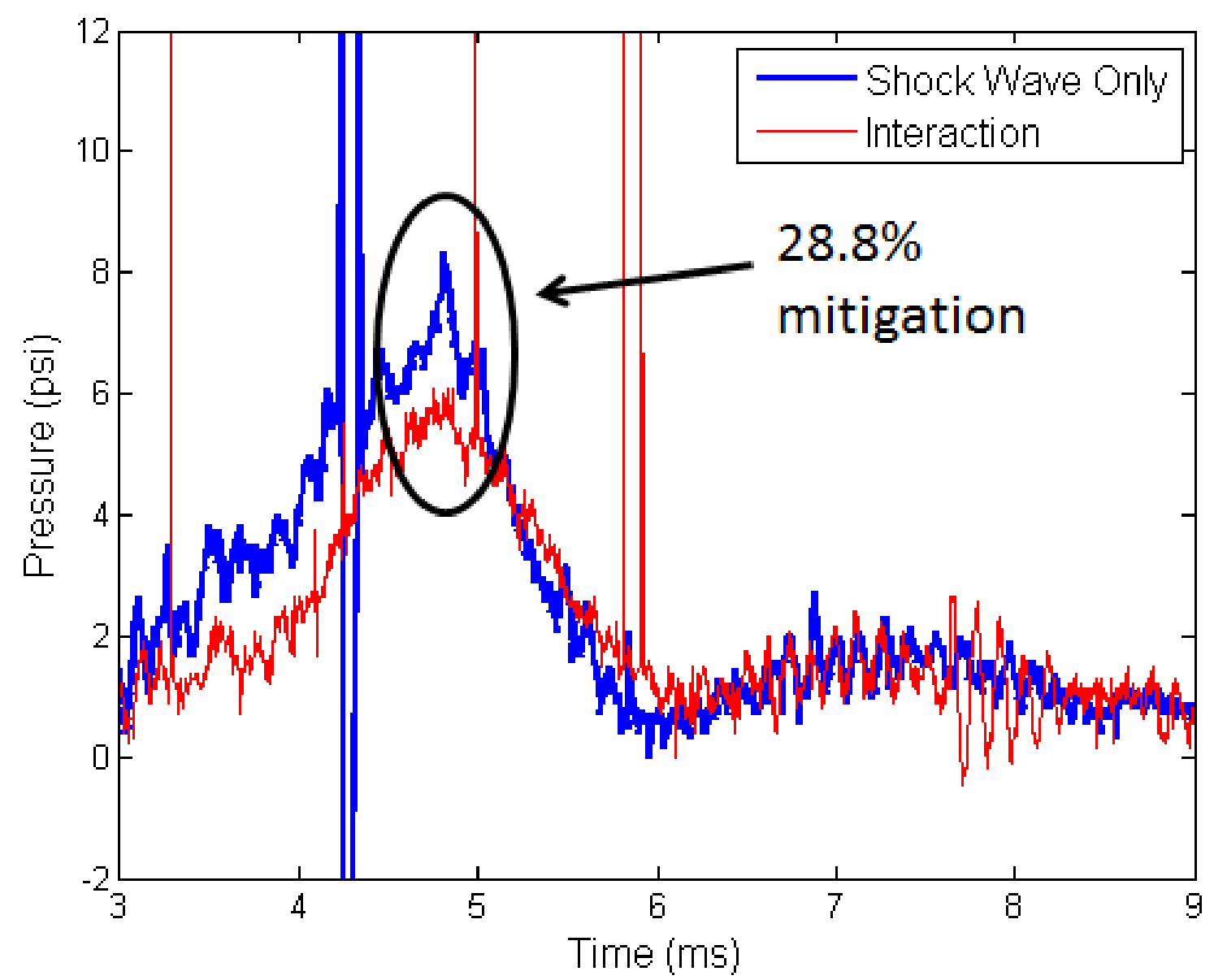

Figure 8.5 An expanded view of the data presented in Figure 8.4. This graph compares an unmitigated shock wave (blue) with a shock wave which has interacted with a TAP discharge (red). A $28.8 \%$ reduction in peak amplitude of the shock wave was observed.

A total of eight shock-wave only experiments and nine interaction experiments were conducted. Seven of the nine interaction experiments were conducted with the nominal TAP discharge (characterized in Chapter 5). For the other two experiments, the shock wave interacted with a larger TAP discharge resulted from removing the resistance in the plasma generation circuit described in Section 6.5.3. This plasma discharge was over ten times as large as the nominal TAP discharge for the first $4 \mathrm{~ms}$ after wire vaporization. This discharge was the largest observed over all experiments conducted. Unfortunately, due to its significantly bigger size and energy, the discharge was also more stressful to the pressure sensors. This discharge damaged one of the two working pressure sensors 
(Channel 3), leaving only one viable pressure sensor. Again, resource constraints of this program did not allow for the sensor to be repaired for use in further experimentation.

Between the two working pressure sensors, the shock wave was reduced in amplitude on 6 of the 7 nominal TAP discharge experiments. There was as much as a $27 \%$ difference in readings between the two sensor positions. On most of the experiments Channel 4 (the sensor placed $0.06 \mathrm{~m}$ off the axis of the exploding wire, to the right) observed the higher reduction in shock wave peak amplitude. On some of the experiments, one of the sensors observed a negative reduction (a gain in peak pressure) but the other sensor (Channel 3, placed $0.06 \mathrm{~m}$ below the axis of the exploding wire) observed a positive reduction. In one experiment, both sensor placements observed negative reduction. The complete amplitude reduction data set is given in Table 8.1.

Table 8.1 Amplitude reduction of shock wave from interaction with the TAP discharge

\begin{tabular}{|r|c|r|r|}
\hline $\begin{array}{c}\text { Experiment } \\
\text { Number }\end{array}$ & $\begin{array}{c}\text { Maximum } \\
\text { Pressure } \\
\text { Reduction } \\
\text { observed }\end{array}$ & $\begin{array}{c}\text { Plasma } \\
\text { Mitigation CH 3 } \\
\text { (low sensor) }\end{array}$ & $\begin{array}{c}\text { Plasma } \\
\text { Mitigation CH 4 } \\
\text { (right sensor) }\end{array}$ \\
\hline 2 & $11.44 \%$ & $\mathbf{1 1 . 4 4 \%}$ & $-3.15 \%$ \\
\hline 3 & $32.96 \%$ & $5.97 \%$ & $\mathbf{3 2 . 9 6 \%}$ \\
\hline 4 & $24.02 \%$ & $12.98 \%$ & $\mathbf{2 4 . 0 2 \%}$ \\
\hline $\mathbf{5 *}$ & $-6.75 \%$ & $-4.34 \%$ & $\mathbf{7 . 7 5 \%}$ \\
\hline 6 & $20.57 \%$ & $-6.63 \%$ & $\mathbf{2 0 . 5 7 \%}$ \\
\hline 7 & $9.40 \%$ & $\mathbf{8 . 7 5 \%}$ & $\mathbf{9 . 4 0 \%}$ \\
\hline Average & $14.22 \%$ & $3.09 \%$ & \\
\hline $\begin{array}{c}\text { Larger Plasma } \\
\text { Experiment } \\
\text { Number }\end{array}$ & $\begin{array}{c}\text { Plasma } \\
\text { (right sensor) }\end{array}$ & & \\
\hline 1 & $48.45 \%$ & & \\
\hline 2 & 50.63\% & & \\
\hline
\end{tabular}


This data is inconsistent, but understandable given the spatial variance in the plasma. We conclude from this data that the toroidal shape of the plasma, and the variation in its drift between experiments, cause a large variance in the spatial overlap between the shock wave and the plasma. This spatial variance, coupled with the limited number of pressure sensors, could have caused the inconsistent observation of peak amplitude reduction. The data would suggest that the plasma was positioned 5 times out of 7 more towards the right sensor (Channel 4). Additionally we suggest that in experiment number 5, the TAP discharge was maligned with the two pressure sensors, causing the gain in pressure of the shock wave. On average, the peak amplitude reduction observed was $14.22 \%$. If experiment number 5 were discarded, the peak amplitude reduction observed increases to

\section{$17.69 \%$}

Additionally, the two trials in which the shock wave interacted with the larger plasma discharge, the mitigation numbers were significantly higher at $48.45 \%$ and $50.63 \%$. It is likely that the larger plasma caused a higher mitigation of the shock wave due to its higher energy in addition to better spatial alignment with the shock wave. This increase in reduction is encouraging for future experiments which should focus on interaction with this enhanced TAP discharge to better characterize the consistency of these results.

\section{References for Chapter 8}

1. A. Lodes, M. Rudroff, R. D. Curry, M. Schmidt, W. Brown, "The Interaction of Shock Waves with High Density Air Plasma," Pulsed Power Conference (PPC), 2011 IEEE, pp.1077-1080, June 2011 


\section{CHAPTER 9: CONCLUSION AND DISCUSSION}

This research program primarily focused on the interaction of shock waves with plasma discharges in air at atmospheric pressure. Developing a suitable plasma discharge with long enough duration (over $1 \mathrm{~ms}$ ) to interact with an externally created shock wave was a significant portion of these efforts. Previous research, reviewed in Chapter 2, has observed mitigation of shock waves as a result of passing through electrical discharges in a variety of low pressure experiments. This research program was unique in that experiments were conducted in ambient air at nominal atmospheric pressure.

While several potential experimental plasma sources were explored, an experimental apparatus was designed and tested to trigger a shock wave from a shock tube. The resultant shock wave exited the tube with a peak amplitude of approximately $10 \mathrm{psi}$.

For interaction with the shock wave, a novel multi-millisecond duration toroidal air plasma, or TAP, was developed. The discharge was initially produced by confining the expansion of a current-driven exploding wire with approximately 100-660 J deposited into the wire by the $4.4 \mathrm{~kJ}, 30 \mathrm{kV}$ RLC pulsed power circuit. The test apparatus designed for these experiments only allows expansion in one axial direction, where an estimated $32 \mathrm{~J}$ of additional energy is deposited in the discharge by an accelerator region via a secondary $8.8 \mathrm{mF}$ capacitor bank storing $275 \mathrm{~J}$.

The TAP has been observed to endure beyond $10 \mathrm{~ms}$ while traveling $0.20 \mathrm{~m}$ or more from the test fixture. The toroidal ring expands to approximately $0.12 \mathrm{~m}$ in diameter. Many efforts of this research program have focused on characterizing the TAP by fully resolving the size and shape throughout its lifetime, characterizing the initial temperature and electron density of the plasma through spectroscopy (approximately $6200 \mathrm{~K}$ and 
electron density of $10^{20} \mathrm{~m}^{-3}$ or $10^{14} \mathrm{~cm}^{-3}$ ), and conducting several experiments to investigate the scalability and optimization of the discharge.

Several experiments showed evidence of increasing the size and duration of the TAP discharge. Doubling the energy stored in the capacitor bank resulted in TAP discharges which were on average 93-177\% larger in area, 27\% longer in duration, and traveled 27\% farther than the typical configuration. A series-wire fixture produced a discharge that was on average $80 \%$ larger in area for the first $1.5 \mathrm{~ms}, 25 \%$ shorter in duration, and $9 \%$ farther in distance traveled, resulting in plasmas that are $46 \%$ faster in velocity than those from the nominal fixture. Changing the wire material to nickel-chromium also resulted in an increase in size of the discharge. The largest TAP discharges produced were observed when removing the discrete resistance in the plasma circuit resulting in an under damped RLC discharge into the wire load. This circuit configuration resulted in a significant increase in energy to the TAP, with $72 \%$ longer duration (up to $20 \mathrm{~ms}$ ) which traveled $76 \%$ farther (up to $0.60 \mathrm{~m}$ ).

A complete mathematical model describing the TAP discharge is not possible at this time as the complex magnetohydrodynamic equations must be solved in air at atmospheric pressure for long durations, requiring computing resources which are beyond the scope of this project. Much of the experimentation with the TAP discharge has been exploratory in nature to attempt to qualitatively and quantitatively characterize certain critical factors such as circuit parameters, wire length, and energy deposited into the circuit. Parameters associated with traditional exploding wire discharges, primarily those effecting current action, have been shown to be important in the size and duration of the discharge. It is the author's expectation that any future research program will allow for 
the design of an optimal toroidal air plasma discharge with many of the conclusions from this project given a set of energy storage and delivery capabilities. It is also expected that any future attempts in modeling this phenomena use the numerical results of these experiments as fitting parameters.

This research program was focused on the interaction of the TAP with the externally produced shock wave. Interaction experiments were designed to examine the interaction of the shock wave with the TAP moments after it detaches from the stationary fixture. In practice, the experiments conducted have timed this interaction at approximately $2,500 \mu \mathrm{s}$ after wire vaporization when the plasma is approximately a torus with a $0.045-0.06 \mathrm{~m}$ major radius and a $0.0175 \mathrm{~m}$ minor radius.

A diagnostic suite including high speed cameras, Schlieren photography and two sets of pressure sensors was used to observe the interaction of the shock wave with the TAP. The plasma momentum stalled and eventually reversed velocity as a result of interacting with the shock wave. Pressure sensors were placed directly 'downstream' of the interaction region to observe the pressure front of the shock wave in conditions with and without the plasma discharge.

Despite a limited data set due to failure of pressure sensors, two sensors were able to observe reduction in shock wave amplitude in 6 of the 7 experiments. The maximum mitigation observed was $32.96 \%$. The variance in this mitigation data is troubling, but understandable when considering spatial and timing constraints of the system. It is likely that with increased repetition and additional pressure sensors more consistent mitigation would be observed. Two additional interaction trials were conducted with the enhanced 
TAP plasma source resulting in mitigation numbers that were significantly higher at $48.45 \%$ and $50.63 \%$.

These experiments confirm the mitigation effect that plasma discharges have on the pressure profile of a shock wave under the conditions of air at atmospheric pressure. These results are valuable to the academic community as no prior experiments had been conducted under these conditions. This work has done little to definitively clear up the proposed mechanisms behind the mitigation effect, but follow-on efforts could potentially use this data in a more complex analysis, particularly if an accurate model of the TAP discharge were developed.

Future additional data could be recorded with modest amounts of follow-up funding to increase diagnostic capabilities. The experiment test bench has been shown to generate reproducible plasmas and shock waves, fulfilling the program requirements. Varying the interaction timing would yield insight into interaction mechanisms.

If significant additional funding were available, this research could potentially progress in any of three parallel ways. Additional interaction experiments and diagnostics could be designed to collect more robust mitigation data to potentially gain further insight in the mechanisms of the plasma mitigation of shock waves in air. Varying the interaction timing would yield valuable insight and data in this pursuit. Further changing the plasma circuit parameters and observing the interaction results would allow for a more convincing argument for mitigation of the shock wave.

Another avenue for follow on work could focus on redesigning the electrical equipment for optimization of the TAP discharge, whether for continued shock wave mitigation experiments or for a novel application. The electrical equipment could be 
redesigned to optimize the size and duration of the TAP and to facilitate faster experiment repetition, perhaps by changing to solid state switching. This type of optimization could easily utilize many of the experimental results obtained in this work as a starting point for a new design.

Finally, another potential focus of future work could be on modeling the TAP discharge. A complete mathematical model of the discharge could reveal many significant scientific findings and potential industrial or scientific applications, such as in fusion research. Modest improvements to the spectroscopy system could greatly improve accuracy and diagnostic capabilities of the spectroscopic measurements. If these type of improvements were made, characterizing the temporal evolution of temperature, electron density, and composition of the plasma would lead to a better understanding of the formation of the toroid shape. If future funding allows, additional duplicate high speed cameras could be configured in multiple views to create a 3D model of the TAP discharge, further characterizing its shape and variability. 


\section{APPENDIX A: EXPLODING WIRE SIMULATION}

The accompanying MATLAB® program was written by Kevin O'Connor at the University of Missouri Columbia and then modified for the application described. The program was written to simulate an RLC circuit with a dynamic resistance, representing the plasma-generating wires. The circuit is depicted in the Figure A.1. The primary energy storage capacitor bank is represented by $C_{\text {Bank }}, L_{\text {Bank }}$, and $R_{\text {Bank. }}$. In addition to the required capacitance of the capacitor bank, the equivalent series resistance and inductance of the capacitor bank can be included in the program if needed. The exploding wire is represented as a variable resistor, $R_{\text {Wire }}$, and an equivalent series inductance, $L_{\text {Wire }}$, that can be included in the simulation or set to zero. Components $R_{\text {Stray }}$ and $L_{\text {Stray }}$ represent any stray circuit resistance and inductance, respectively, as well as any discrete resistors or inductors placed in the circuit external to the capacitor bank and exploding wire.

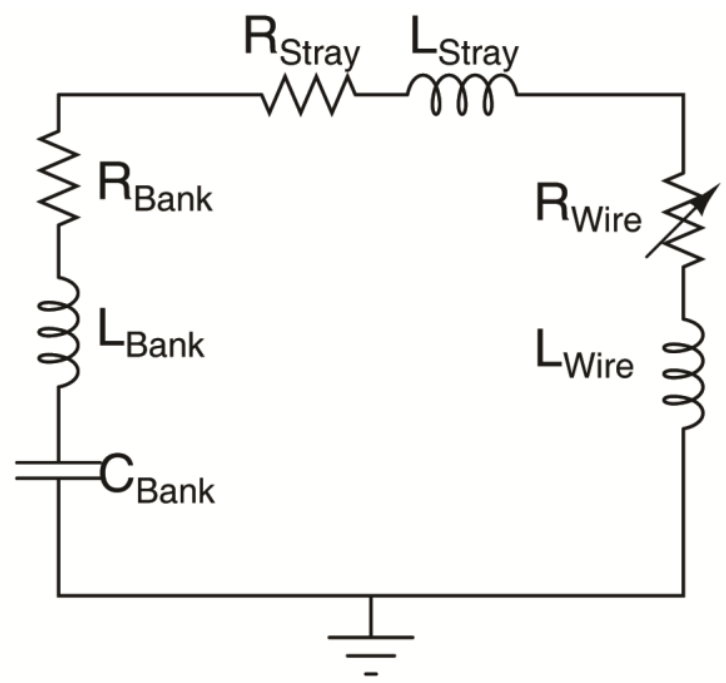

Figure A.1 Simulated Circuit

The program was adapted from programs originally developed to model exploding wire fuses [1]. However, there is one major difference between the case of the plasma 
source and the fuse. Since the fuse is meant to act as an opening switch, restrike or breakdown of the wire is normally avoided entirely. The region of interest in the resistance profile in that case is from room-temperature resistance through vaporization. An approximation of the fuse resistance was created for the fuse program to estimate the resistance behavior during this time, but it is not precisely known. In the case of the plasma source, breakdown of the wire seems to happen very early because expansion of the wire is unconstrained in atmospheric pressure air, and an arc seems to form within a few microseconds. At this point of arc formation, the approximation for restrike resistance is used until the resistance falls to a pre-determined final arc resistance. The majority of the resistance collapse is on the order of nanoseconds to tens of nanoseconds. The remainder of the simulation is essentially an overdamped RLC discharge.

Several of the key simulation and experimental parameters are given in the following table.

\begin{tabular}{c|c|c|c} 
Wire Length $(\mathbf{m})$ & Capacitance $(\boldsymbol{\mu} \mathbf{F})$ & Inductance $(\boldsymbol{\mu} \mathbf{H})$ & Resistance $(\mathbf{\Omega})$ \\
\hline 0.1016 & 11 & 12 & 5.2
\end{tabular}

\begin{tabular}{c|c} 
Number of Wires & Charging Voltage (kV) \\
\hline 2 & 30
\end{tabular}


The following is the exploding wire simulation program written in MATLAB $®$.

Copyright statement: The program is a copyright of Kevin A. O'Connor [1]. It has

been modified for this research by Mike Rudroff and Adam Lodes for the research

presented in this dissertation.

\section{References for Appendix A:}

1. K. A. O'Connor, "Compact Power Conditioning and RF Systems for a High Power RF Source," Ms. Thesis, Elec. and Comp. Engr., University of Missouri, Columbia, MO, 2008.

$\% \% \% \% \% \% \% \% \% \% \% \% \% \% \% \% \% \% \% \% \% \% \% \% \% \% \% \% \% \% \% \% \% \% \% \% \% \% \% \% \% \% \% \% \% \% \% \% \% \% \%$ $\% \% \% \% \% \% \% \% \% \% \% \% \% \% \% \% \% \% \%$

$\% \% \%$ The following program will modify an RLC circuit with a varying

$\% \% \%$ resistance $\% \% \%$

$\% \% \%$ from an exploding wire fuse. The following parameters can be

$\% \% \%$ modified to best represent the circuit under consideration.

$\% \% \%$ Written by Kevin O'Connor.\%

$\% \% \%$ Modified by Michael Rudroff and Adam Lodes

$\% \% \% \% \% \% \% \% \% \% \% \% \% \% \% \% \% \% \% \% \% \% \% \% \% \% \% \% \% \% \% \% \% \% \% \% \% \% \% \% \% \% \% \% \% \% \% \% \% \% \%$

$\% \% \% \% \% \% \% \% \% \% \% \% \% \% \% \% \% \% \% \%$

clear;

close all;

clc;

outputoptions= $\left[\begin{array}{llllllll}1 & 1 & 1 & 1 & 1 & 1 & 0 & 0\end{array}\right]$; \% Automatic plot selection: 1 for plot. 0 for no plot.

$\%$ 1.Current 2.Fuse Resistance 3.Fuse Voltage 4. High Voltage Probe 5.

$\%$ Capacitor Bank Voltage 6.Efuse

NumberofCaps $=5 ; \%$ Number of capacitors

Ccap=2.2E-6; \% Capacitance of each capacitor $[\mathrm{F}]$

ChargingVoltage $=30 \mathrm{E} 3$; \% Initial charging voltage of each capacitor [V]

Lbank=1.5E-9; \% Equivalent inductance of capacitor bank $[\mathrm{H}]$

Lfuse $=15.4 \mathrm{E}-9 ; \%$ Inductance of fuse

Lstray=12E-6; \% Stray or other circuit inductance $[\mathrm{H}]$

Rresistor $=5.2 ; \%$ Stray or other circuit resistance [Ohm]

Rbank $=0 ; \%$ Effective resistance of capacitor bank [Ohm] - currently unknown

singlewirearea $=4.87 \mathrm{E}-9$; \% Area of single fuse wire $\left[\mathrm{m}^{\wedge} 2\right]$

numberofwires $=2 ; \%$ Total number of parallel fuse wires

wirelength $=.1016 ; \%$ Fuse wire length [m]

wiremassdensity $=8690 ; \%$ Wire mass density $\left[\mathrm{kg} /\left(\mathrm{m}^{\wedge} 3\right)\right]$

initialwireresistivity $=1.725 \mathrm{E}-8 ; \%$ Room temperature wire resistivity [Ohm-m]

fuseshortresistance $=0.005 ; \%$ Final resistance of fuse after restrike [Ohm]

Esp1=1409000; \% Specific energy at action limit [J/kg]

Esp2=5909000; \% Specific energy at burst $[\mathrm{J} / \mathrm{kg}]$

fusebreakdownstrength=400000; \% Breakdown field of fuse vapor [V/m]. Select 1E12 to disregard.

$\mathrm{A}=23.9$; \% Fuse resistivity curve-fitting parameter - 23.9 for copper

$\mathrm{B}=2.3 ; \%$ Fuse resistivity curve-fitting parameter -2.3 for copper

$\mathrm{C}=118 ; \%$ Fuse resistivity curve-fitting parameter -118 for copper

alpha $=2.8 \mathrm{E}-9 ; \%$ Fuse material parameter $\left[\left(\mathrm{m}^{\wedge} 2\right) / \mathrm{A} /\left(\mathrm{s}^{\wedge} .5\right)\right]$

tstep $=3 \mathrm{E}-9 ; \%$ Time between data points. Must be kept small.

tstop $=150 \mathrm{E}-6 ; \%$ End time of simulation 
numSteps=tstop/tstep;

\%electron density counter

ElDensity=0;

electronDensity $(1,1)=0$;

\%volume of containment cylinder:

VolumeCyl=1.2847E-5; \%(m^3)

\%ionization constants for Copper:

FirstIon $=745400 ; \quad \%(\mathrm{~J} / \mathrm{mol})$

SecondIon $=1957900 ; \%(\mathrm{~J} / \mathrm{mol})$

ThirdIon $=3553500 ; \quad \%(\mathrm{~J} / \mathrm{mol})$

Atomize $=338000 ; \quad \%(\mathrm{~J} / \mathrm{mol})$

atomicMass $=.063546 ; \%(\mathrm{~kg} / \mathrm{mol})$

Navagadro $=6.022 \mathrm{E}+23$;

Wphase $=0 ; \quad \%$ phase of wire $(0=$ solid, $1=$ liquid, $2=$ vapor $)$

$\%$ Spitzer resistivity:

SpitzerRes=8.1E-5;

PlasmaWidth $(1,1)=0$;

$\% \% \% \% \% \% \% \% \% \% \% \% \% \% \% \% \% \% \% \% \% \% \% \% \% \% \% \% \% \% \% \% \% \% \% \% \% \% \% \% \% \% \% \% \% \% \% \% \% \% \%$ $\% \% \% \% \% \% \% \% \% \% \% \% \% \% \% \% \% \% \% \% \% \% \% \% \% \% \% \% \%$

$\% \% \%$ The rest of the program is not to be modified under normal circumstances $\% \% \%$

$\% \% \% \% \% \% \% \% \% \% \% \% \% \% \% \% \% \% \% \% \% \% \% \% \% \% \% \% \% \% \% \% \% \% \% \% \% \% \% \% \% \% \% \% \% \% \% \% \% \% \%$ $\% \% \% \% \% \% \% \% \% \% \% \% \% \% \% \% \% \% \% \% \% \% \% \% \% \% \% \% \%$

CBank=Ccap $*$ NumberofCaps;

Ltotal=Lbank+Lfuse+Lstray;

Rstray=Rresistor+Rbank;

totalwirearea=numberofwires*singlewirearea;

fusemass=totalwirearea* wirelength* wiremassdensity;

\%number of moles:

Num_moles=fusemass* $(1 /$ atomicMass $)$;

$\%$ number of atoms:

Num_atoms=Num_moles*Navagadro;

$\%$ Energies needed to ionize:

Eatomize $=$ Atomize $*$ Num_moles;

Efirst $=($ FirstIon*Num_moles $)+$ Eatomize;

Esecond $=($ SecondIon*Num_moles $)+$ Efirst;

Ethird=(ThirdIon*Num_moles)+Esecond;

he $=(\text { totalwirearea/alpha })^{\wedge} 2$;

$\mathrm{X}=0$;

$\mathrm{t}(1,1)=0$;

$\mathrm{h}(1,1)=0$;

$\operatorname{rho}(1,1)=$ initialwireresistivity;

$\operatorname{Rf}(1,1)=\operatorname{rho}(1,1) *$ wirelength/totalwirearea;

$\mathrm{R} 0=\operatorname{Rf}(1,1)$;

$\mathrm{I}(1,1)=0$;

$\mathrm{EC}(1,1)=.5 * \mathrm{CBank}^{*}\left((\text { Charging Voltage })^{\wedge} 2\right)$;

$\mathrm{EL}(1,1)=0$;

Efuse $(1,1)=0$;

$\operatorname{Erstray}(1,1)=0$; 
EfusePerMass $(1,1)=$ Efuse $(1,1) /$ fusemass;

$\mathrm{E} 0=\mathrm{EL}(1,1)+\mathrm{Efuse}(1,1)+\mathrm{EC}(1,1)+\operatorname{Erstray}(1,1)$;

EConservedTest $(1,1)=(\operatorname{EL}(1,1)+\operatorname{Erstray}(1,1)+\operatorname{Efuse}(1,1)+\mathrm{EC}(1,1)-\mathrm{E} 0) / \mathrm{E} 0$;

Vrfuse $(1,1)=0$;

Prfuse $(1,1)=$ Vrfuse $(1,1) * \mathrm{I}(1,1)$;

Vlfuse $(1,1)=0$;

Vfusetotal $(1,1)=\operatorname{Vrfuse}(1,1)+\operatorname{Vlfuse}(1,1)$;

$\operatorname{Vrstray}(1,1)=0$;

Prstray $(1,1)=0$;

$\mathrm{Vc}(1,1)=$ Charging Voltage;

Vprobe $(1,1)=0$;

$\mathrm{b}(1,1)=(\operatorname{Rf}(1,1)+$ Rstray $) /($ Ltotal $)$;

$\mathrm{c}=1 /\left(\mathrm{CBank}^{*}(\right.$ Ltotal $\left.)\right)$;

$\mathrm{s} 1(1,1)=.5^{*}\left(-\mathrm{b}(1,1)+\operatorname{sqrt}\left(\left(\mathrm{b}(1,1)^{\wedge} 2\right)-(4 * \mathrm{c})\right)\right)$;

$\mathrm{s} 2(1,1)=.5^{*}\left(-\mathrm{b}(1,1)-\operatorname{sqrt}\left(\left(\mathrm{b}(1,1)^{\wedge} 2\right)-(4 * \mathrm{c})\right)\right)$;

$\operatorname{dIdt}(1,1)=(1 /(\mathrm{s} 2(1,1)-\mathrm{s} 1(1,1))) *\left(\left(\mathrm{I}(1,1) *\left(\left(\left(\mathrm{~s} 2(1,1)^{\wedge} 2\right) * \exp (\mathrm{s} 2(1,1) * \mathrm{t}(1,1))\right)-\right.\right.\right.$

$\left.\left.\left(\left(\operatorname{s} 1(1,1)^{\wedge} 2\right)^{*} \exp (\mathrm{s} 1(1,1) * \mathrm{t}(1,1))\right)\right)\right)+((\operatorname{Vc}(1,1) /(\operatorname{Ltotal})) *((\mathrm{~s} 2(1,1) * \exp (\mathrm{s} 2(1,1) * \mathrm{t}(1,1)))-$

$(\mathrm{s} 1(1,1) * \exp (\mathrm{s} 1(1,1) * \mathrm{t}(1,1))))))$;

$\mathrm{i}=2$;

while $\mathrm{t}(1, \mathrm{i}-1)<$ tstop;

$\mathrm{t}(1, \mathrm{i})=\mathrm{t}(1, \mathrm{i}-1)+\mathrm{tstep}$

$\mathrm{I}(1, \mathrm{i})=\mathrm{I}(1, \mathrm{i}-1)+(\operatorname{tstep} * \operatorname{dIdt}(1, \mathrm{i}-1))$;

$\mathrm{I} 2(1, \mathrm{i})=\mathrm{I}(1, \mathrm{i})^{\wedge} 2$;

$\mathrm{h}(1, \mathrm{i})=\mathrm{h}(1, \mathrm{i}-1)+(\mathrm{I} 2(1, \mathrm{i}-1) * \operatorname{tstep})+((.5 *(\mathrm{I} 2(1, \mathrm{i})-\mathrm{I} 2(1, \mathrm{i}-1)) * \operatorname{tstep}))$;

$\mathrm{Vc}(1, \mathrm{i})=\mathrm{Vc}(1, \mathrm{i}-1)-((1 / \mathrm{CBank}) *(\operatorname{tstep} *(\mathrm{I}(1, \mathrm{i}-1)+(0.5 *(\mathrm{I}(1, \mathrm{i})-\mathrm{I}(1, \mathrm{i}-1))))))$;

$\operatorname{Vrfuse}(1, \mathrm{i})=\mathrm{I}(1, \mathrm{i}) * \operatorname{Rf}(1, \mathrm{i}-1)$;

Prfuse $(1, \mathrm{i})=\left(\operatorname{Vrfuse}(1, \mathrm{i})^{\wedge} 2\right) / \operatorname{Rf}(1, \mathrm{i}-1)$;

Efuse $(1, \mathrm{i})=$ Efuse $(1, \mathrm{i}-1)+(\operatorname{tstep} *(\operatorname{Prfuse}(1, \mathrm{i}-1)+(.5 *(\operatorname{Prfuse}(1, \mathrm{i})-\operatorname{Prfuse}(1, \mathrm{i}-1)))))$;

\%Electron Density Calculation:

if (Efuse $(1, \mathrm{i})>=$ Eatomize $) \& \&$ (Efuse $(1, \mathrm{i})<$ Efirst $)$;

Wphase $=1$;

elseif (Efuse $(1, \mathrm{i})>=$ Efirst $) \& \&$ (Efuse $(1, \mathrm{i})<$ Esecond);

Wphase $=2$;

ElDensity=Num_atoms/VolumeCyl; \%(1 electron/m^3)

elseif (Efuse $(1, \mathrm{i})>=$ Esecond $) \& \&(\operatorname{Efuse}(1, \mathrm{i})<$ Ethird);

Wphase $=3$;

ElDensity=(2*Num_atoms $) /$ VolumeCyl; \%(2 electrons $\left./ \mathrm{m}^{\wedge} 3\right)$

elseif (Efuse $(1, \mathrm{i})>=$ Ethird);

Wphase $=4$;

ElDensity $=(3 *$ Num_atoms $) /$ VolumeCyl; $\%\left(3\right.$ electron/ $\left./ \mathrm{m}^{\wedge} 3\right)$

end;

electronDensity $(1, \mathrm{i})=$ ElDensity;

\%Plasma Width Calculation:

PlasmaWidth $(1, \mathrm{i})=(($ SpitzerRes*wirelength $) / \operatorname{Rf}(1, \mathrm{i}-1))$;

EfusePerMass $(1, \mathrm{i})=$ Efuse $(1, \mathrm{i}) /$ fusemass;

$\mathrm{EL}(1, \mathrm{i})=.5^{*}\left(\mathrm{I}(1, \mathrm{i})^{\wedge} 2\right)^{*}($ Ltotal $)$;

$\mathrm{EC}(1, \mathrm{i})=.5^{*} \mathrm{CBank} *\left(\mathrm{Vc}(1, \mathrm{i})^{\wedge} 2\right)$;

$\operatorname{Prstray}(1, \mathrm{i})=(\mathrm{I}(1, \mathrm{i}) * \mathrm{I}(1, \mathrm{i})) *$ Rstray;

$\operatorname{Erstray}(1, \mathrm{i})=\operatorname{Erstray}(1, \mathrm{i}-1)+(\operatorname{tstep} *(\operatorname{Prstray}(1, \mathrm{i}-1)+(.5 *(\operatorname{Prstray}(1, \mathrm{i})-\operatorname{Prstray}(1, \mathrm{i}-1)))))$;

if $\mathrm{h}(1, \mathrm{i})<=\mathrm{he}$;

$\operatorname{rho}(1, \mathrm{i})=\left(1+\left(\mathrm{A} *\left((\mathrm{~h}(1, \mathrm{i}) / \mathrm{he})^{\wedge} \mathrm{B}\right)\right)\right)$; 


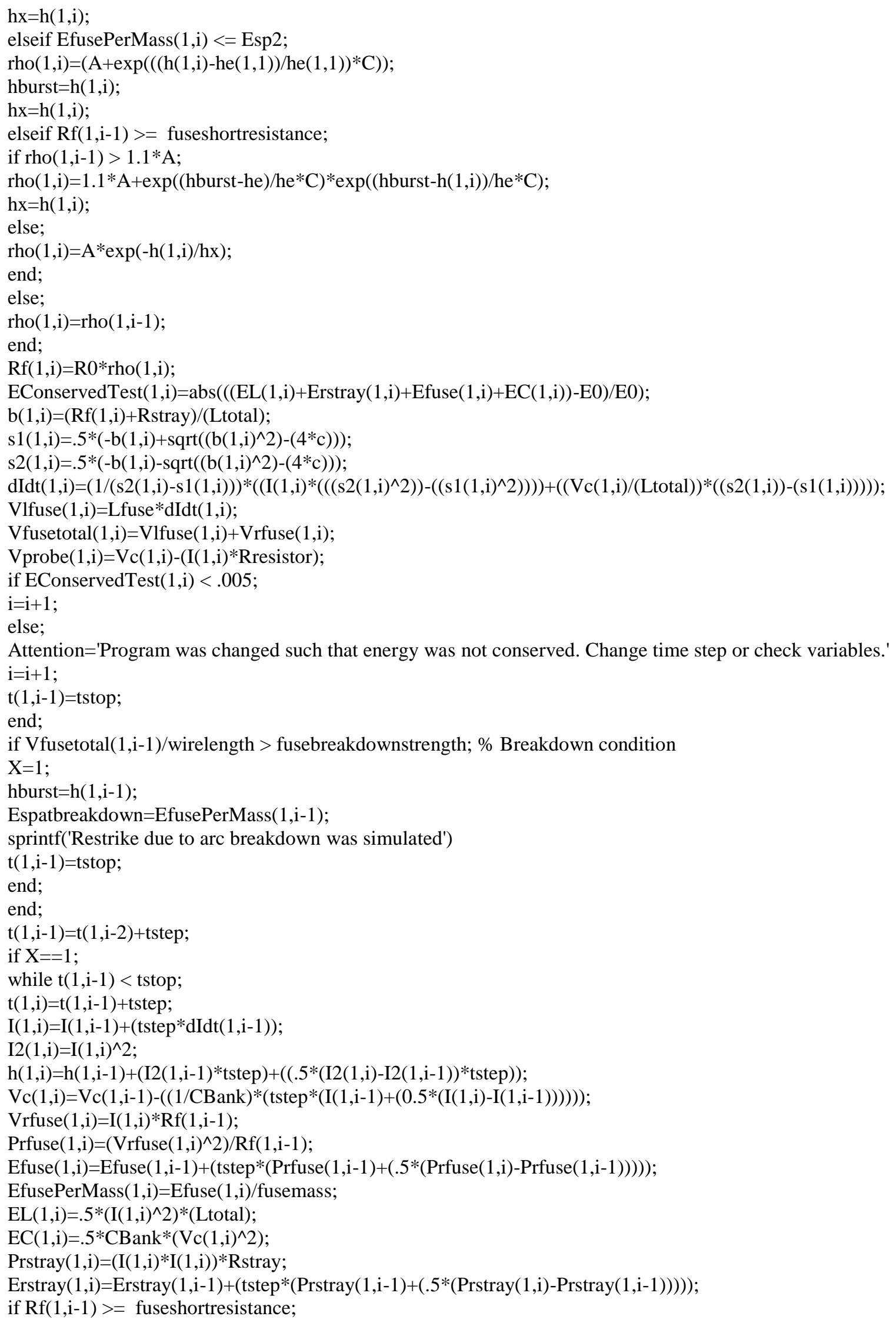




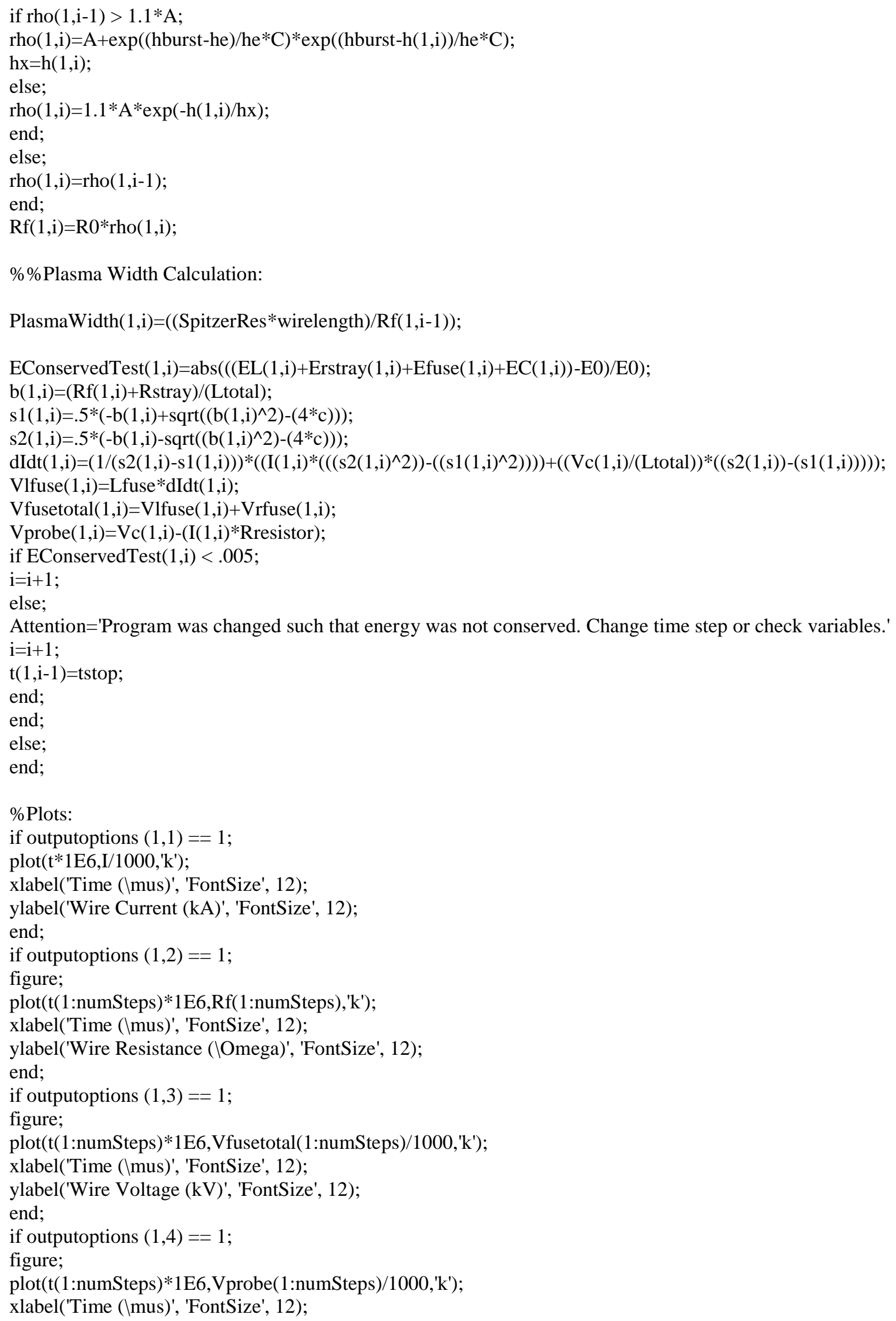


ylabel('High Voltage Probe (kV)', 'FontSize', 12);

end;

if outputoptions $(1,5)==1$;

figure;

$\operatorname{plot}\left(\mathrm{t}(1:\right.$ numSteps $) * 1 \mathrm{E} 6, \mathrm{Vc}(1:$ numSteps $\left.) / 1000, \mathrm{k}^{\prime}\right)$;

xlabel('Time (lmus)', 'FontSize', 12);

ylabel('Capacitor Bank Voltage (kV)', 'FontSize', 12);

end;

\%Fuse energy:

if outputoptions $(1,6)==1$;

figure;

plot(t(1:numSteps)*1E6,Efuse(1:numSteps),'k');

xlabel('Time (lmus)', 'FontSize', 12);

ylabel('Energy Deposited(J)', 'FontSize', 12);

end;

\%electron density:

if outputoptions $(1,7)==1$;

figure;

$\operatorname{plot}(\mathrm{t}(1$ :numSteps)*1E6,electronDensity(1:numSteps),'k');

xlabel('Time (lmus)', 'FontSize', 12);

ylabel('Electron Density (e-/m^3)', 'FontSize', 12);

end;

\%Plasma width:

if outputoptions $(1,8)==1$;

figure;

plot(t(1:numSteps)*1E6,PlasmaWidth(1:numSteps),'k');

xlabel('Time (\mus)', 'FontSize', 12);

ylabel('Plasma Width (m)', 'FontSize', 12);

end; 


\section{APPENDIX B: HIGH VOLTAGE TRIGGER CIRCUIT AND TIMING DIAGRAM}

Triggering the spark gap breakdown was critical in the timing of the plasma and the shock wave. The trigger circuit was designed to be reliable and consistent, while being cost efficient and relatively compact. The high voltage trigger circuit delivers a high voltage pulse to the trigger plane of the spark gap of up to $45 \mathrm{kV}$, causing switch breakdown. Duplicate independent triggering circuits were used to trigger the plasma circuit and the shock tube circuit.

The high voltage trigger circuit diagram is shown in Figure B.1. The high voltage pulse is delivered by an MSD 6420 capacitive discharge ignition system, which sends a $460 \mathrm{~V}$ pulse into a 100:1 oil-filled MSD Blaster 2 ignition coil. These components are designed to operate the spark gaps in an automobile's ignition system. The MSD 6420 requires a constant $12 \mathrm{~V}$ power source, and will output its $460 \mathrm{~V}$ pulse when its ignition wire is switched to ground. A MPS222A npn type transistor is employed to accomplish this upon receiving a $+\mathrm{TTL}$ pulse. The output of the transformer coil is placed in series with a $50 \Omega$ ceramic high-voltage resistor and a $673 \mathrm{pF}$ isolating capacitor. The isolating capacitor is connected to the trigger plane of the pressurized T-670 switch with dual $50 \mathrm{M} \Omega$ resistors used to bias the trigger plane of the spark gap with respect to the electrodes. The trigger circuit is contained within a stainless steel junction box for safety and RF shielding. The circuit is shown implemented in Figure B.2. 


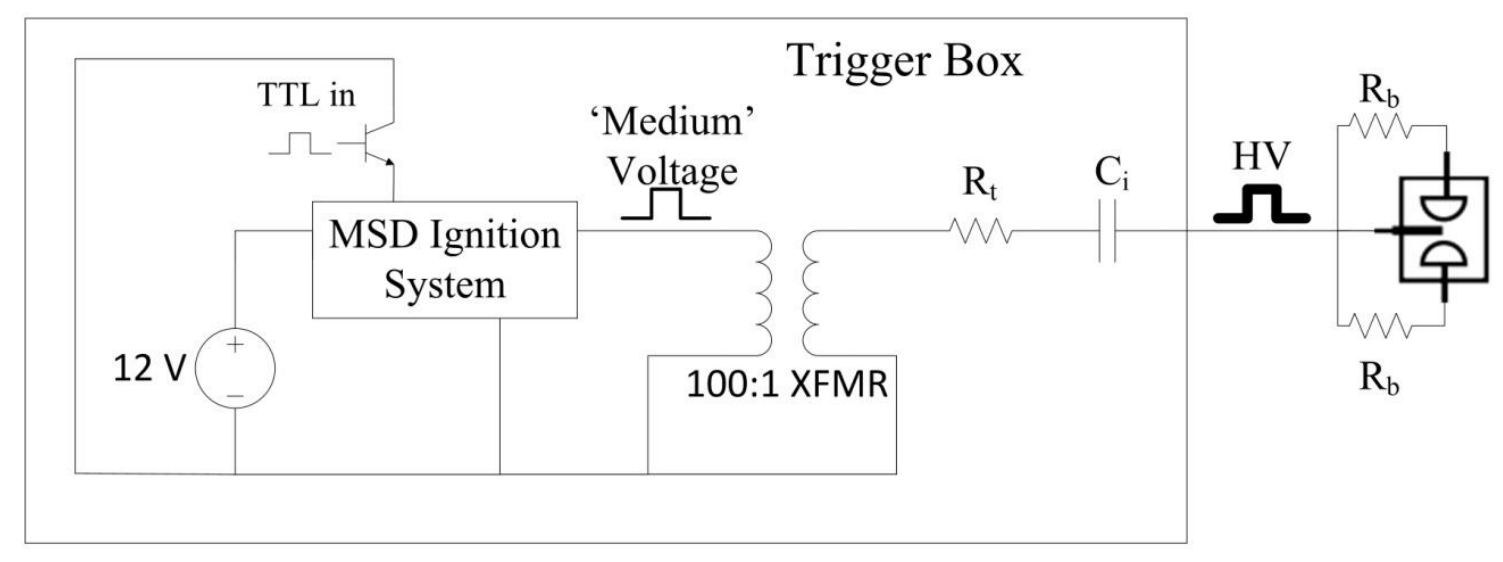

Figure B.1 Diagram of high voltage trigger circuit

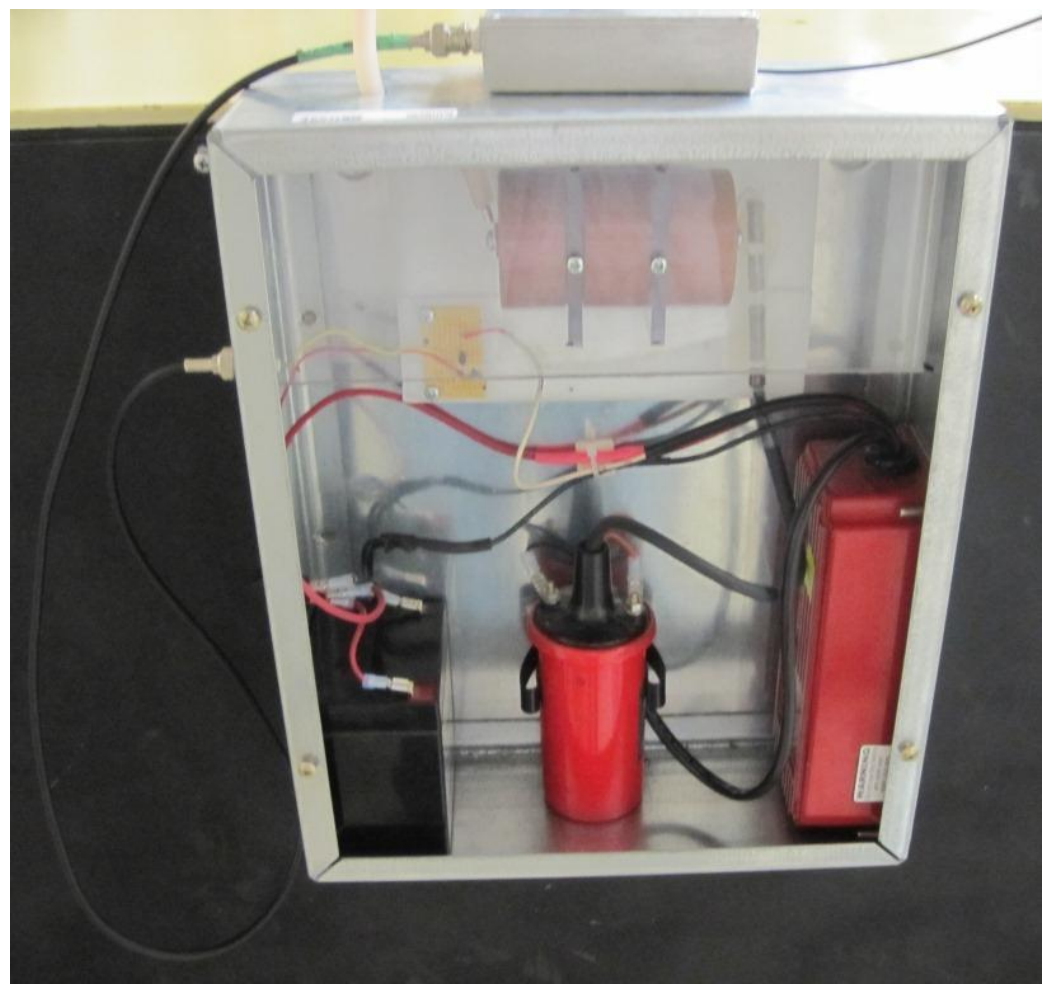

Figure B.2 High voltage trigger circuit

The shock tube circuit and plasma circuit are triggered separately with an arbitrary timing delay by the SRS DG645 digital delay generator. The shock tube circuit and all diagnostics are triggered upon a push of a button on the delay generator. This manual initiation is referred to as ' $t \_0$ ' and all trigger signals in this discussion are referenced to 
this time unless otherwise noted. The timing diagram, Figure B.3, shows the timing of various events in the triggering of the experiment. The high voltage trigger circuits have a practical switch time of about $2,150 \mu \mathrm{s}$. As explained previously, this is the time from the delay generator output signal to the high voltage trigger pulse at the third plate in the spark gap. The majority of this delay occurs in the circuitry of the MSD itself. This switching delay is denoted 't_msd_sw' for the MSD switching delay for the shock tube circuit, and 't_msd_pl' for the plasma circuit in Figure B.3.

The initial trigger, t_0, enables the MSD for the shock tube circuit, and the MSD switches $2,150 \mu \mathrm{s}$ later. At this time, breakdown occurs in the spark gap for the shock tube circuit, causing the energy stored in the capacitor bank to discharge into the circuit and the dynamic wire load. The wire explodes and ruptures the diaphragm of the shock tube in about $10 \mu \mathrm{s}$ ('t_cir_sw' in Figure B.3). Once the diaphragm is ruptured, the shock wave exits the shock tube and arrives $0.19 \mathrm{~m}$ from the end of the tube approximately $6,762 \mu \mathrm{s}$ from t_0. This formation and transit time of the shock wave is denoted 't_form\&trans_sw' in Figure B.3.

The TAP discharge must also arrive in this location at this time. This location has been referred to as the 'interaction region,' and each high speed camera has been configured to have the entire region in its field of view. The time of interaction, $\mathrm{t} \_$interaction, is $6,762 \mu \mathrm{s}$ from t_0. 


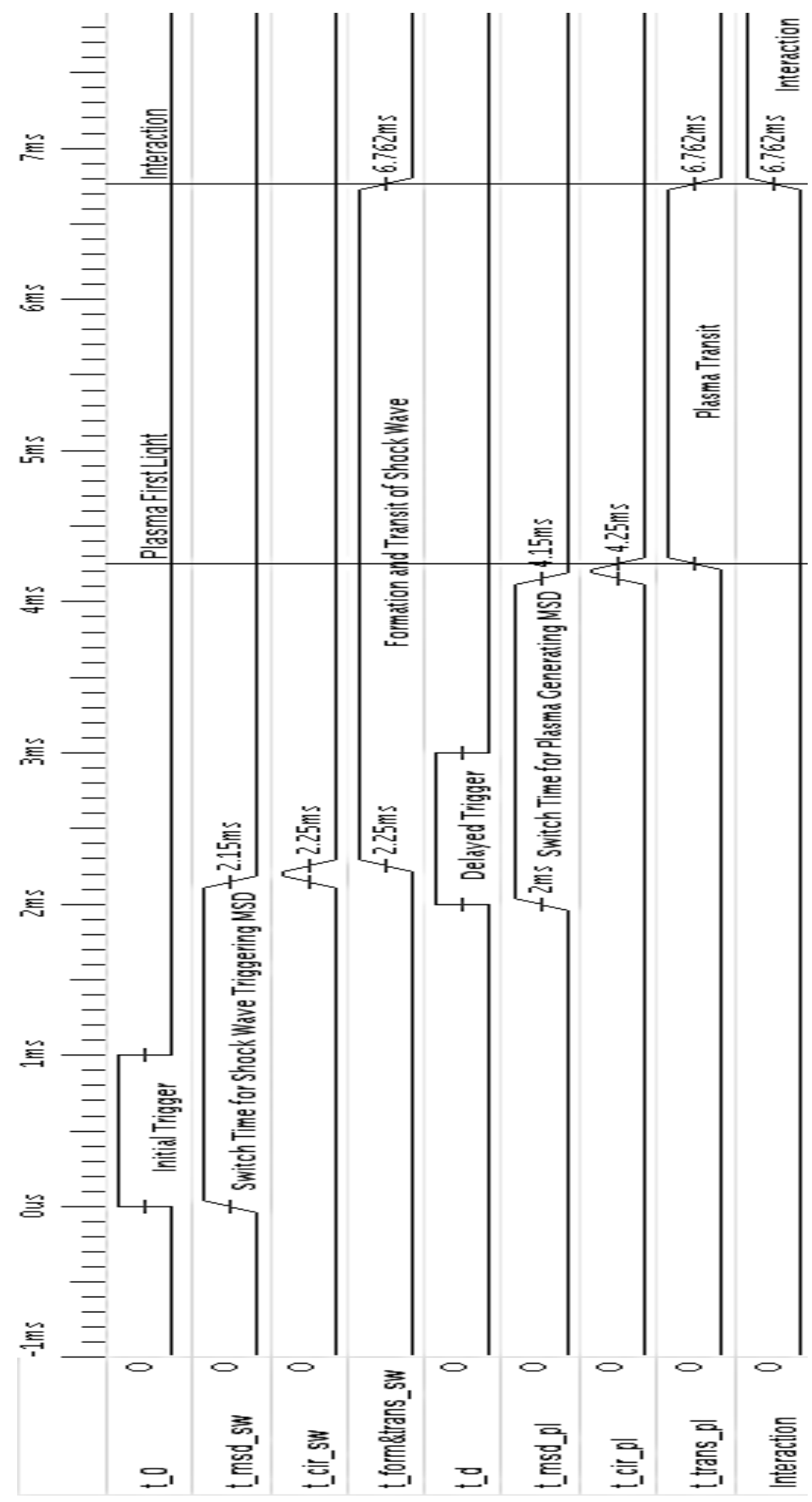

Figure B.3 Timing diagram for experiments 
The plasma arrives at the interaction region about $2,500 \mu$ s from wire vaporization. Without delay the TAP would arrive at the interaction region much earlier than the shock wave. Thus, for interaction to occur within the observable interaction region, a timing delay, 't_d', of $2,000 \mu$ s must be added to the channel which triggers the plasma circuit. This delayed signal triggers the MSD circuit for plasma generation, and 2,150 $\mu$ s later (t_msd_pl), the spark gap of the plasma circuit closes from the high voltage pulse applied to its third plate. The energy stored in the capacitors is discharged into the circuit. This causes the wire to explode and produce light in about $10 \mu \mathrm{s}$ ('t_cir_pl'). The plasma then expands out of the test fixture toward the interaction region, as discussed in Chapter 5, over the time 't $t$ trans_pl'. At interaction, the plasma was observed to be $0.11-0.12 \mathrm{~m}$ in height, and 0.08-0.10 $\mathrm{m}$ in width. 


\section{APPENDIX C: IMAGE PROCESSING ALGORITHM}

For further analysis of the high speed videos of the TAP, a program was created in MATLAB® to track the height, width, area, and distance traveled of the plasma throughout its lifetime.

Some basic assumptions have been made to facilitate this analysis. Each video is scrutinized to the same processing algorithm. Before analysis, each movie file must be converted from the native .cine file to an .avi file to be recognized by MATLAB ${ }$. This conversion decreases the resolution from the original 16 bit video to an 8 bit avi file. The .avi movie file is split into individual RGB images. The algorithm converts each RGB image to a grayscale image, and then to a binary image based on a predetermined threshold. At this point, each pixel within each image is either a 0 or a 1 , either dark or light.

Typical experiments have the plasma fixture oriented in the left side of the view of the camera with the plasma propagating toward the right. As the algorithm converts each image of a movie to a binary image, it tracks the size and location of the plasma. This is accomplished by tracking the largest 'blob' of pixels which are lit. If there are multiple blobs signifying a plasma that is inhomogeneous the algorithm chooses the largest to follow. Because the threshold for determining each pixel's value ( 0 or 1$)$ is discrete, the algorithm will be subject to discrete transitions as the TAP dissipates. Often in the later stages of propagation of the TAP, the plasma cools unevenly and some parts are brighter than others in appearance. When watching a video, dissipation is a smooth transition. The program is jagged in the transitions, as seen by sharp sudden decreases in the size of the 
plasma blob. While the algorithm is jagged in this fashion it typically only occurs when the TAP is dissipating, thus the algorithm generates a good barometer of when the TAP begins to cool. More advanced image processing techniques could be employed to more accurately smooth these edges in the future, but knowing when the TAP begins to dissipate is useful at this time.

The algorithm is also designed to ignore any pixels at or behind the plasma fixture. Typically the experiments result in a stationary secondary discharge at the accelerator region which endures longer than the TAP. This analysis is not interested in the stationary discharge, thus the program ignores these pixels.

As previously discussed, the program tracks the height, width, area, and distance traveled of the TAP through time. These calculations are all accomplished by tracking the largest blob of illuminated pixels. The tallest column within the blob is counted as the height and the widest row of pixels within the blob is the width. Thus, these values are the maximum values of the TAP discharge at a given time. An example of the analysis is shown in Figure C.1. The area of the TAP is calculated by counting the number of pixels illuminated. The distance over which the plasma has traveled is calculated by tracking the horizontal position of the tallest column within the blob (the same column by which the height is calculated). This horizontal coordinate is subtracted from the reference position of the plasma fixture. 


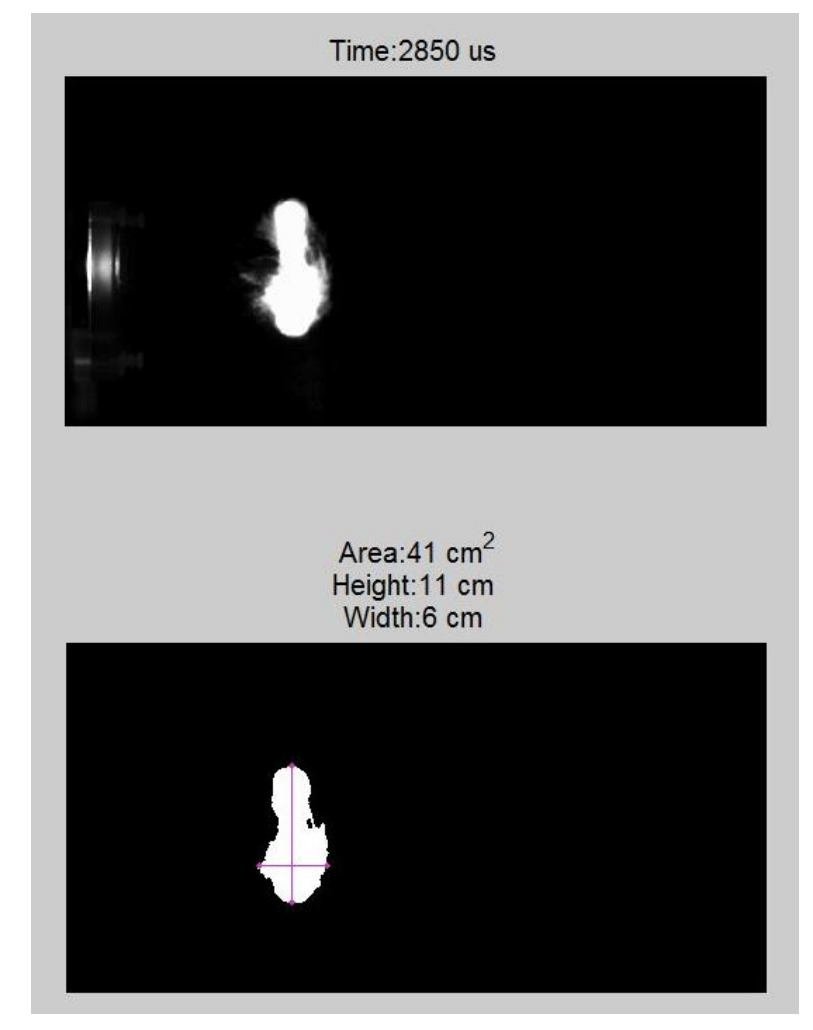

Figure C.1 Example of image processing analysis of the TAP

These calculations are then subject to a correction factor which calibrates the pixel distance with actual distance in centimeters. Prior to running the program, these calibration factors are entered as part of the input block as the widthScale and heightScale. This scale is adjusted on each position of the high speed camera, and is calculated based on the fixed size of the plasma fixture. The input block also requires the filename, fname, of the avi video, the frame rate of the high speed video, termed cineFR (default is 21052), the reference position in a pixel coordinate termed startPoint of the plasma fixture, and the binary threshold parameter, termed levell (default is 0.4).

Many output options are available. A two-window movie can be created which compares the original video unprocessed and the processed video with visuals associated 
with the tracking of the TAP. An example of a frame of this movie is shown in Figure C.1. This video is displayed at runtime, but it by default not saved, the option to save this video is commented out at the end of the program. The height, width, area, and distance traveled over time of the TAP are plotted in separate figures at the end of the program. These figures are displayed, buy by default not saved. Again, the option to save these figures is commented out at the end of the program.

The image processing algorithm was further modified to analyze the size and shape of the TAP from the on-axis perspective. This version of the program tracks the minor radius of the TAP. Each image is scanned for the thickness of the toroid. Once the toroid has formed, there are sections of the plasma which have two blobs of lit pixels for each row and column of the toroid. These two blobs are averaged, for each row and each column respectively, then the row value and the column value are averaged to arrive at a toroid thickness. This thickness is divided in half to arrive at an approximation of the toroid minor radius. This calculation is done for each image.

Further MATLAB® codes are used to calculate the major radius from an average of the height and width of the TAP in this on-axis perspective. The average of the height and width of the TAP is divided in half and the minor radius (previously calculated) is then subtracted from this value to obtain an approximation of the toroid major radius. This final correction is to utilize the definition of the major radius of a toroid (shown in Figure 4.10). Each image of each video from the on-axis perspective was processed by this modified algorithm to track the minor and major radii of the TAP. 
The remainder of Appendix $\mathrm{C}$ are the MATLAB ${ }^{\circledR}$ codes which implement these image processing algorithms, starting with the initial program where the camera is viewing the TAP from the side-axis perspective.

Copyright statement: The program is a copyright of Adam Lodes for this research. Use of the program by researchers is welcomed as long as credit for authorship is appropriately noted.

close all;

clear

clc;

$\%$ Video Analyze is meant to analyze movies of the TAP

$\%$ this program tracks the height, width, and size of the plasma

$\%$ it also calculates the distance traveled of the plasma

$\%$ There are several necessary inputs

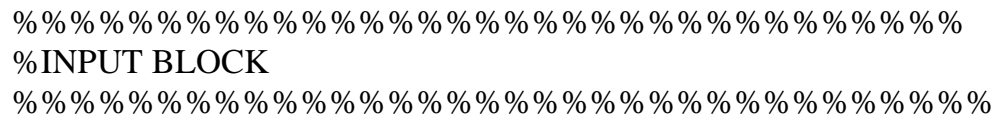

$\% \% \% \%$ PROGRAM PARAMETER, level1 is a threshold for gray or white $\%$ threshold is 0 to 1 . 1 allows only highest value pixel (bright) $\% 0$ allows all but pixel value 0 ; $\% \%$ This is a critical parameter for identifying the plasma 


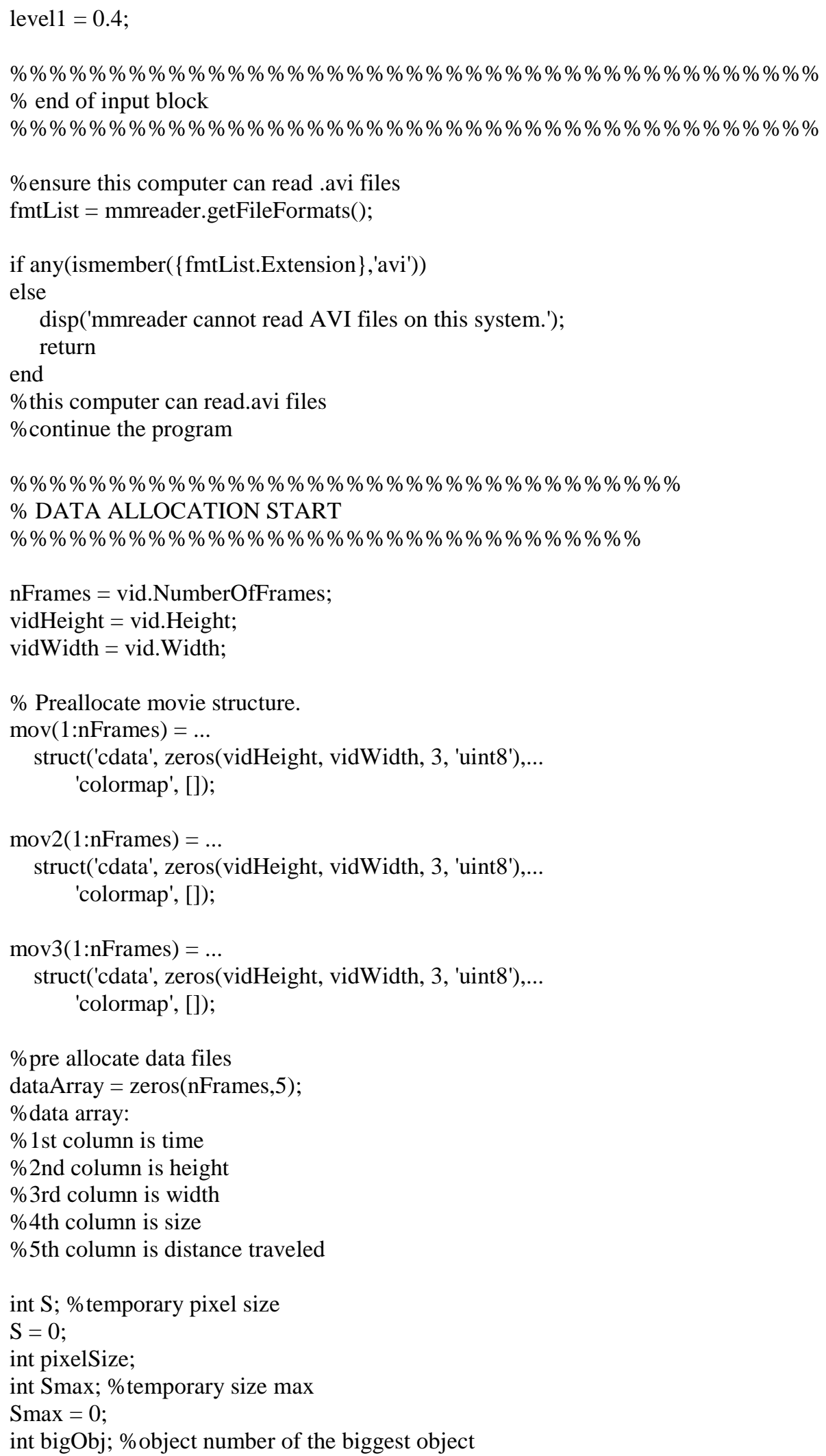




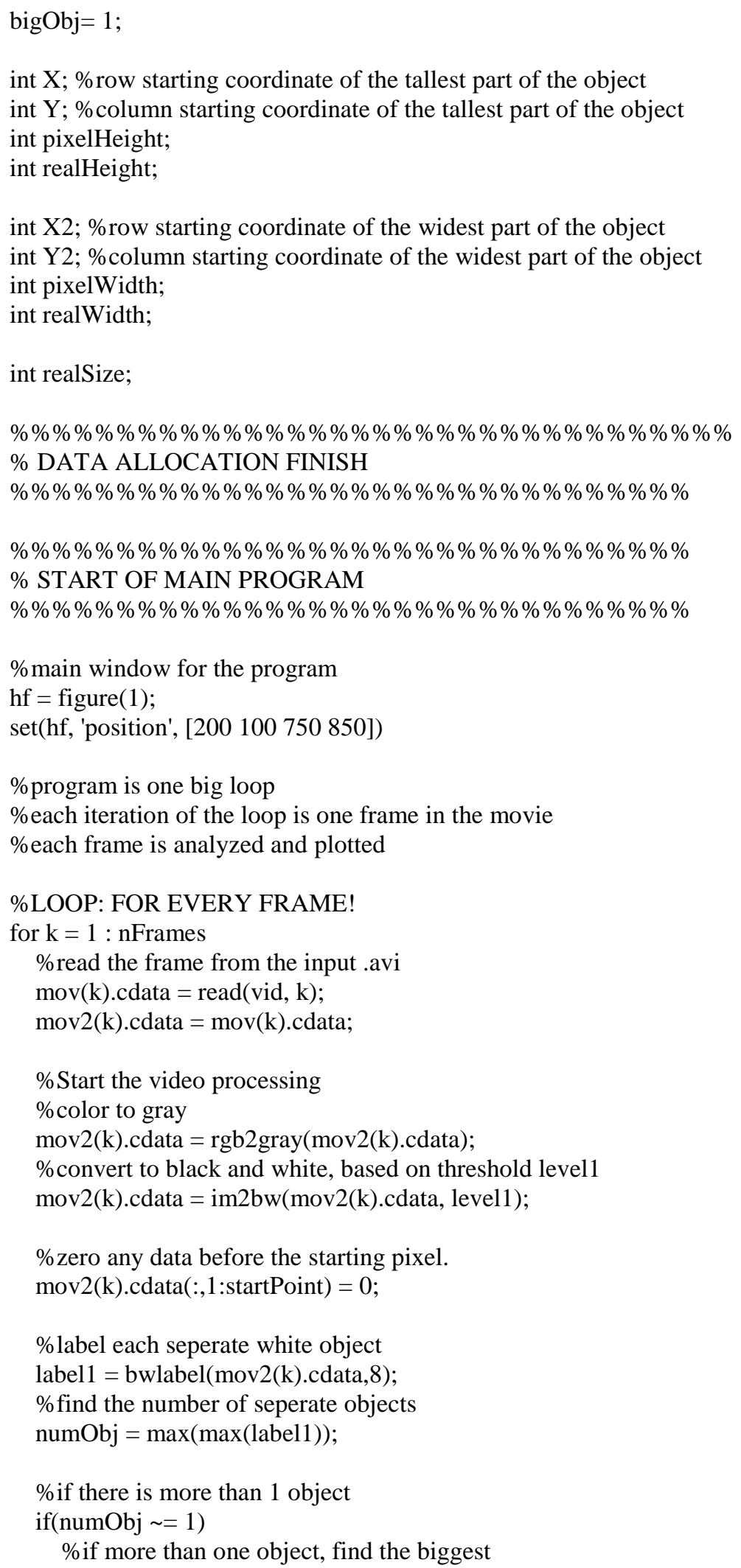




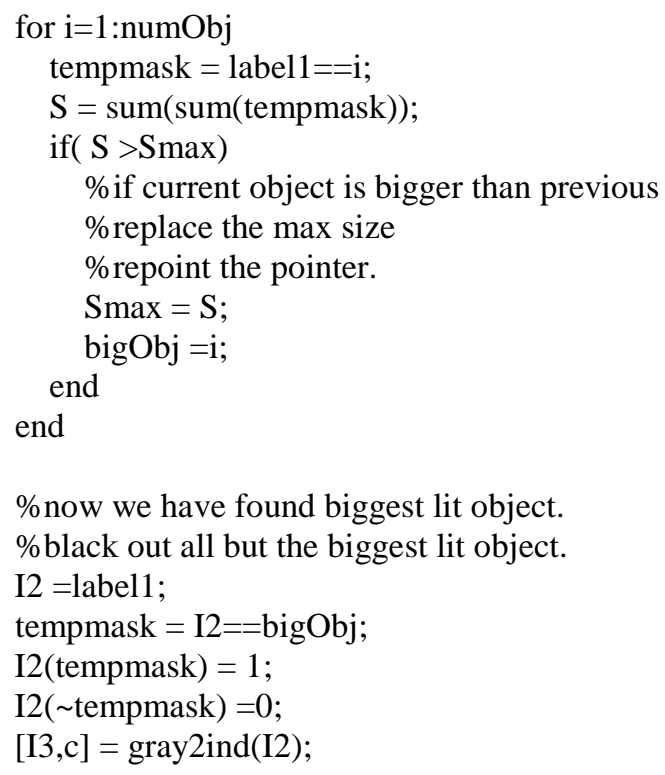

else \%if only 1 object is found

\%turn the image back to a format that is easily put into a movie

I2 =label1;

$[\mathrm{I} 3, \mathrm{c}]=\operatorname{gray} 2 \mathrm{ind}(\mathrm{I} 2)$;

end

$\% \%$ calculate the pixel size of the label2 = bwlabel(I3,8);

tempmask2 = label2==1;

pixelArea $=\operatorname{sum}(\operatorname{sum}($ tempmask2 $))$;

$\%$ I4 is untouched movie

$\mathrm{I} 4=\operatorname{mov}(\mathrm{k}) \cdot \mathrm{cdata}$;

$\%$ the title of the top figure, just Time

tempstr= strcat('Time: ', int $2 \mathrm{str}\left(\mathrm{k}^{*} \mathrm{dt}\right)$, , us');

$\%$ plot the current frame of the movie, edited and non-edited

$\%$ show the original image up top

$\%$ show the processed image on bottom

subplot(2,1,1), imshow(I4);

title(tempstr,'FontSize', 15);

$\operatorname{subplot}(2,1,2)$, imshow $(\mathrm{I} 3, \mathrm{c})$;

\%find and draw the tallest and widest part of the plasma

$[\mathrm{X}, \mathrm{Y}$, pixelHeight $]=\operatorname{maxHeight}(\mathrm{I} 3$, vidHeight, vidWidth $)$;

line([X,X],[Y,Y+pixelHeight],'Marker','.,'LineStyle','-','LineWidth',1,'Color',[.8 .2 .8])

$[\mathrm{X} 2, \mathrm{Y} 2$, pixelWidth $]=\max$ Width $(\mathrm{I} 3$, vidHeight, vidWidth $)$;

line([X2,X2+pixelWidth],[Y2,Y2],'Marker',,'.,'LineStyle','-','LineWidth',1,'Color',[.8 .2 .8])

\%calculate the area, width and height in $\mathrm{cm}$ (not pixels) 


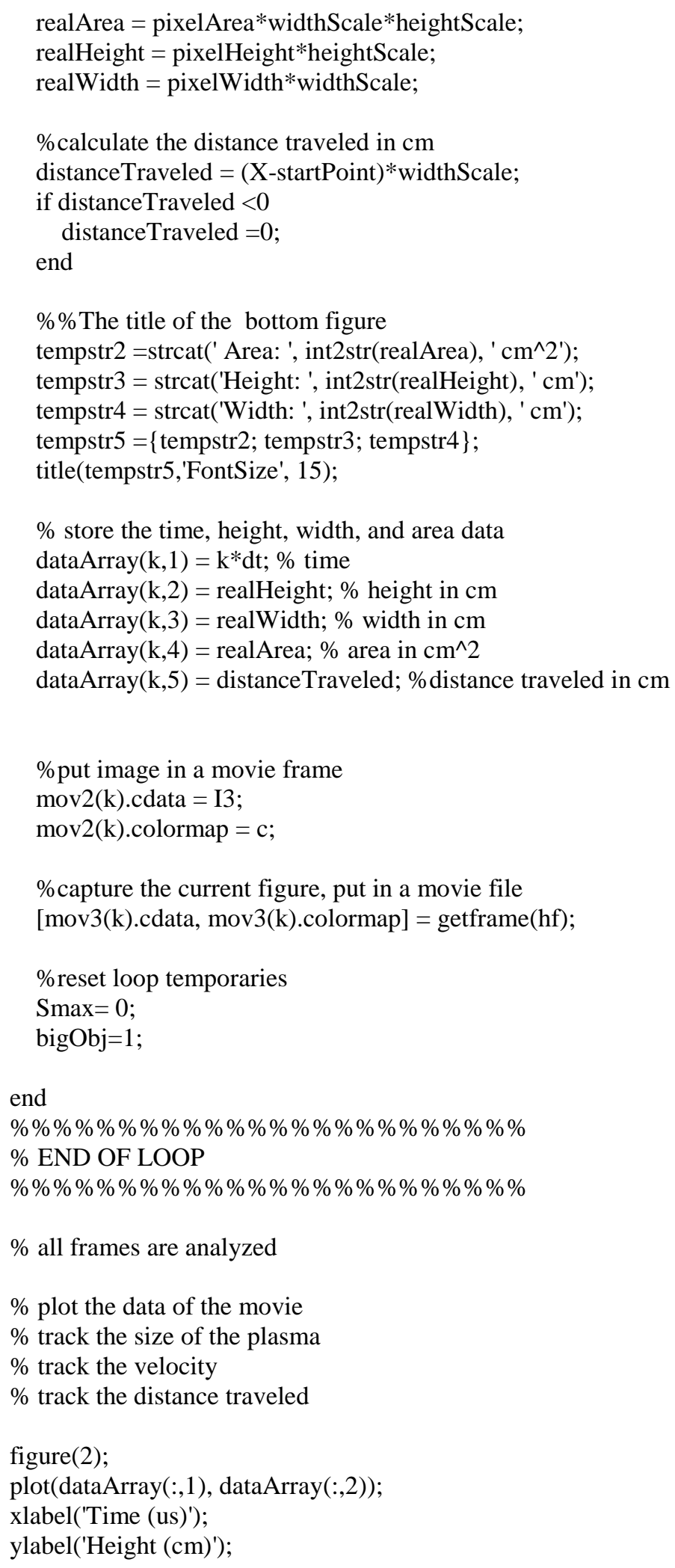




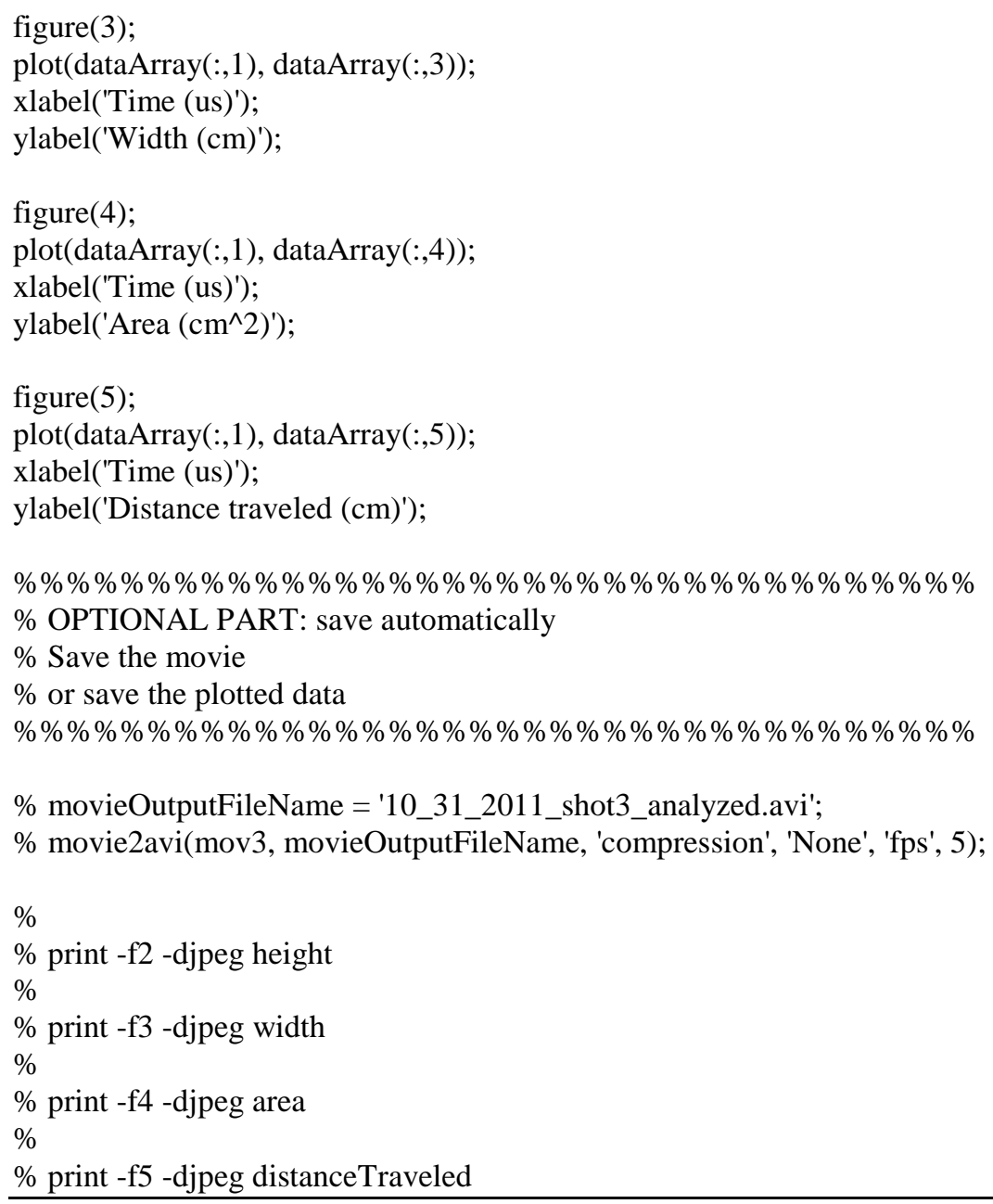

The following is the modified version of the image processing algorithm which tracks the average minor radius of the TAP.

Copyright statement: The program is a copyright of Adam Lodes for this research program. Use of the program by researchers is welcomed as long as credit for authorship is appropriately noted.

close all;

clear

clc;

$\%$ Video Analyze 3 is meant to analyze movies of the TAP

$\%$ this program tracks the height, width, and size of the plasma

$\%$ it also calculates the minor radius of the plasma

$\%$ There are several necessary inputs 
$\% \% \% \% \% \% \% \% \% \% \% \% \% \% \% \% \% \% \% \% \% \% \% \% \% \% \% \% \% \%$

$\%$ INPUT BLOCK

$\% \% \% \% \% \% \% \% \% \% \% \% \% \% \% \% \% \% \% \% \% \% \% \% \% \% \% \% \% \% \% \%$

\%input file name

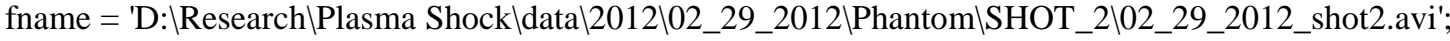

vid $=$ mmreader(fname);

$\% \% \%$ program will error if file doesnt exist

$\%$ frame rate of the Cine

cineFR $=21052 ; \%$ original framerate of the Cine

$\mathrm{dt}=1 / 21052 * 1000000 ; \%$ delta $\mathrm{t}$ of Cine, in us;

$\%$ scale of the width, in $\mathrm{cm}$ per pixel

widthScale $=5.898 * 2.54 / 142$;

\%scale of the height, in $\mathrm{cm}$ per pixel

heightScale $=6.01 * 2.54 / 143$;

$\% \% \%$ PROGRAM PARAMETER, level1 is a threshold for gray or white

$\%$ threshold is 0 to 1 . 1 allows only highest value pixel (bright)

$\% 0$ allows all but pixel value 0 ;

$\% \%$ This is a critical parameter for identifying the plasma

level1 = 0.4;

$\% \% \% \% \% \% \% \% \% \% \% \% \% \% \% \% \% \% \% \% \% \% \% \% \% \% \% \% \% \% \% \% \% \% \% \% \% \% \%$

$\%$ end of input block

$\% \% \% \% \% \% \% \% \% \% \% \% \% \% \% \% \% \% \% \% \% \% \% \% \% \% \% \% \% \% \% \% \% \% \% \% \% \% \% \% \%$

\%ensure this computer can read avi files

fmtList $=$ mmreader.getFileFormats () ;

if any(ismember(\{fmtList.Extension $\}, ' a v i '))$

else

disp('mmreader cannot read AVI files on this system.');

return

end

\%this computer can read.avi files

$\%$ continue the program

$\% \% \% \% \% \% \% \% \% \% \% \% \% \% \% \% \% \% \% \% \% \% \% \% \% \% \% \% \% \% \% \% \% \%$

$\%$ DATA ALLOCATION START

$\% \% \% \% \% \% \% \% \% \% \% \% \% \% \% \% \% \% \% \% \% \% \% \% \% \% \% \% \% \%$

nFrames $=$ vid.NumberOfFrames;

vidHeight $=$ vid.Height;

vidWidth = vid.Width; 


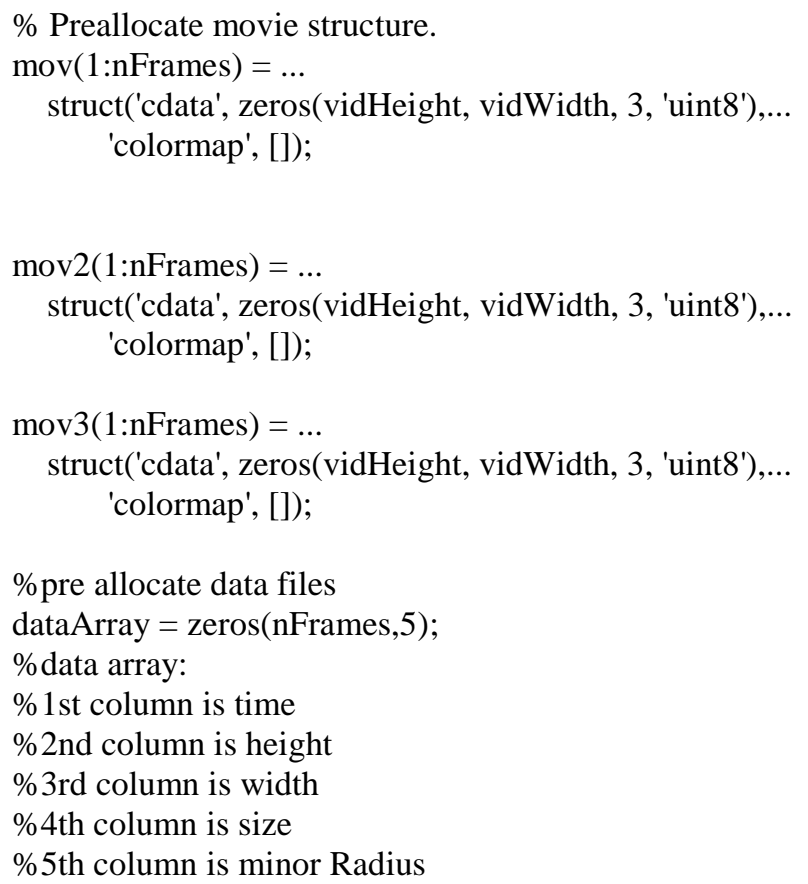

int X; \%row starting coordinate of the tallest part of the object int Y; \%column starting coordinate of the tallest part of the object int pixelHeight;

int realHeight;

int X2; \%row starting coordinate of the widest part of the object int Y2; \%column starting coordinate of the widest part of the object int pixelWidth;

int realWidth;

int realSize;

double pixelMinorDW;

double realMinorDW;

double pixelMinorDH;

double realMinorDH;

double avgMinorD;

$\% \% \% \% \% \% \% \% \% \% \% \% \% \% \% \% \% \% \% \% \% \% \% \% \% \% \% \% \% \% \% \% \% \%$

$\%$ DATA ALLOCATION FINISH

$\% \% \% \% \% \% \% \% \% \% \% \% \% \% \% \% \% \% \% \% \% \% \% \% \% \% \% \% \% \% \% \%$ 


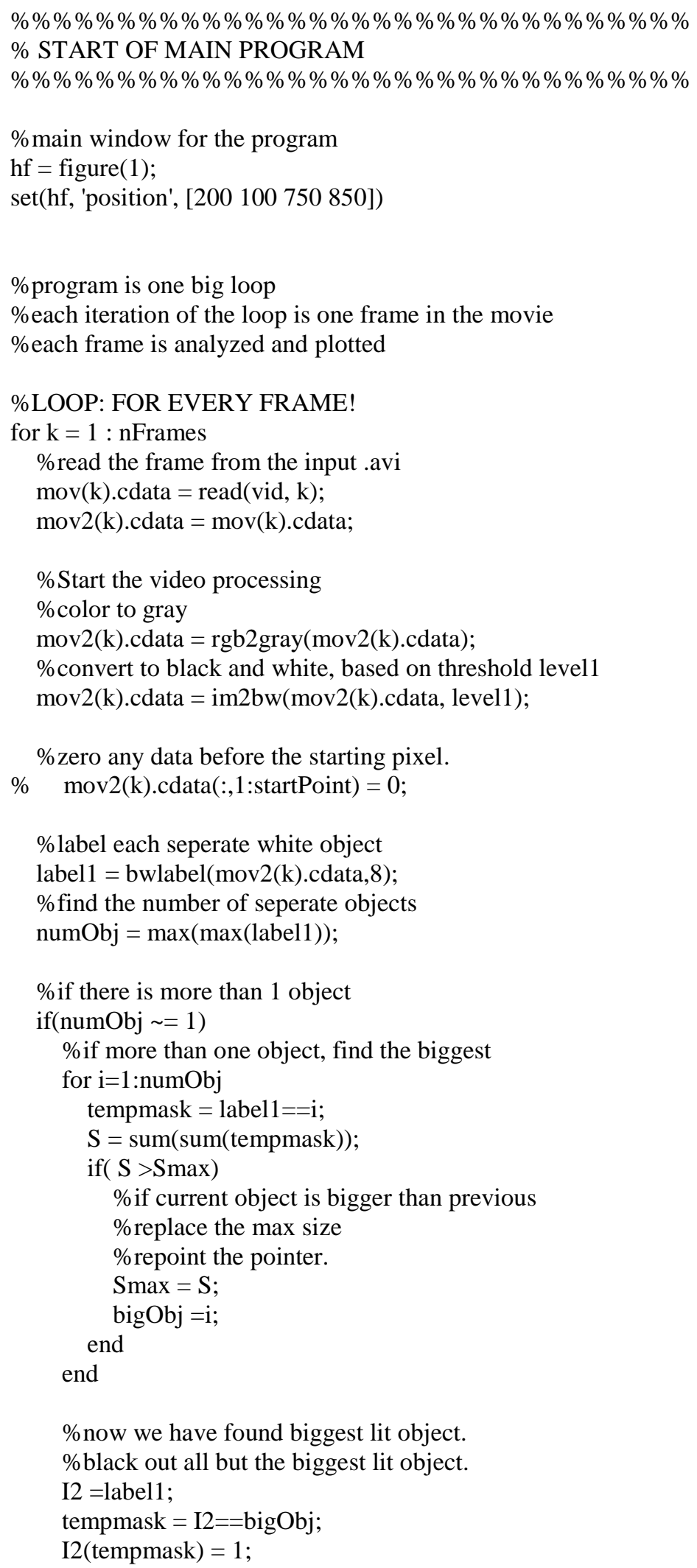


I2( tempmask $)=0$;

$[\mathrm{I} 3, \mathrm{c}]=$ gray2ind $(\mathrm{I} 2)$;

else \%if only 1 object is found

$\%$ turn the image back to a format that is easily put into a movie

I2 =label1;

$[\mathrm{I} 3, \mathrm{c}]=\operatorname{gray} 2 \mathrm{ind}(\mathrm{I} 2)$;

end

$\% \%$ calculate the pixel size of the

label2 = bwlabel $(\mathrm{I} 3,8)$;

tempmask $2=$ label2 $==1$;

pixelArea $=\operatorname{sum}(\operatorname{sum}($ tempmask2 $))$;

$\%$ I4 is untouched movie

$\mathrm{I} 4=\operatorname{mov}(\mathrm{k}) \cdot \mathrm{cdata}$;

$\%$ the title of the top figure, just Time

tempstr= strcat('Time: ', int2str(k*dt), ' us');

$\%$ plot the current frame of the movie, edited and non-edited

\%show the original image up top

\%show the processed image on bottom

subplot(2,1,1), imshow(I4);

title(tempstr,'FontSize', 15);

subplot( $2,1,2)$, imshow(I3,c);

\%find and draw the tallest and widest part of the plasma

$[\mathrm{X}, \mathrm{Y}, \mathrm{pixelHeight}]=\operatorname{maxHeight}(\mathrm{I} 3$, vidHeight, vidWidth);

line([X,X],[Y,Y+pixelHeight],'Marker','.','LineStyle','-','LineWidth',1,'Color',[.8 .2 .8])

$[\mathrm{X} 2, \mathrm{Y} 2$, pixelWidth $]=\max$ Width $(\mathrm{I} 3$, vidHeight, vidWidth $)$;

line([X2,X2+pixelWidth],[Y2,Y2],'Marker','.','LineStyle','-','LineWidth',1,'Color',[.8 .2 .8])

\%avgMinorWidth and avgMinorHeight track the Thickness of the TAP ring

[pixelMinorDW] = avgMinorWidth(I3);

$[$ pixelMinorDH] $=\operatorname{avgMinorHeight}(\mathrm{I} 3)$;

\%calculate the area, width and height in $\mathrm{cm}$ (not pixels)

realArea $=$ pixelArea ${ }^{*}$ widthScale $*$ heightScale;

realHeight $=$ pixelHeight $*$ heightScale;

realWidth $=$ pixelWidth* widthScale;

realMinorDW $=$ pixelMinorDW $*$ widthScale;

realMinorDH $=$ pixelMinorDH ${ }^{*}$ heightScale;

avgMinorD $=($ realMinorDW+realMinorDH $) / 2$;

avgMinorR $=\operatorname{avgMinorD} / 2 ; \%$ want radius not diameter

$\% \%$ The title of the bottom figure

tempstr2 =strcat(' Area: ', int2str(realArea), ' cm^2');

tempstr3 = strcat('Height: ', int2str(realHeight), ' cm'); 


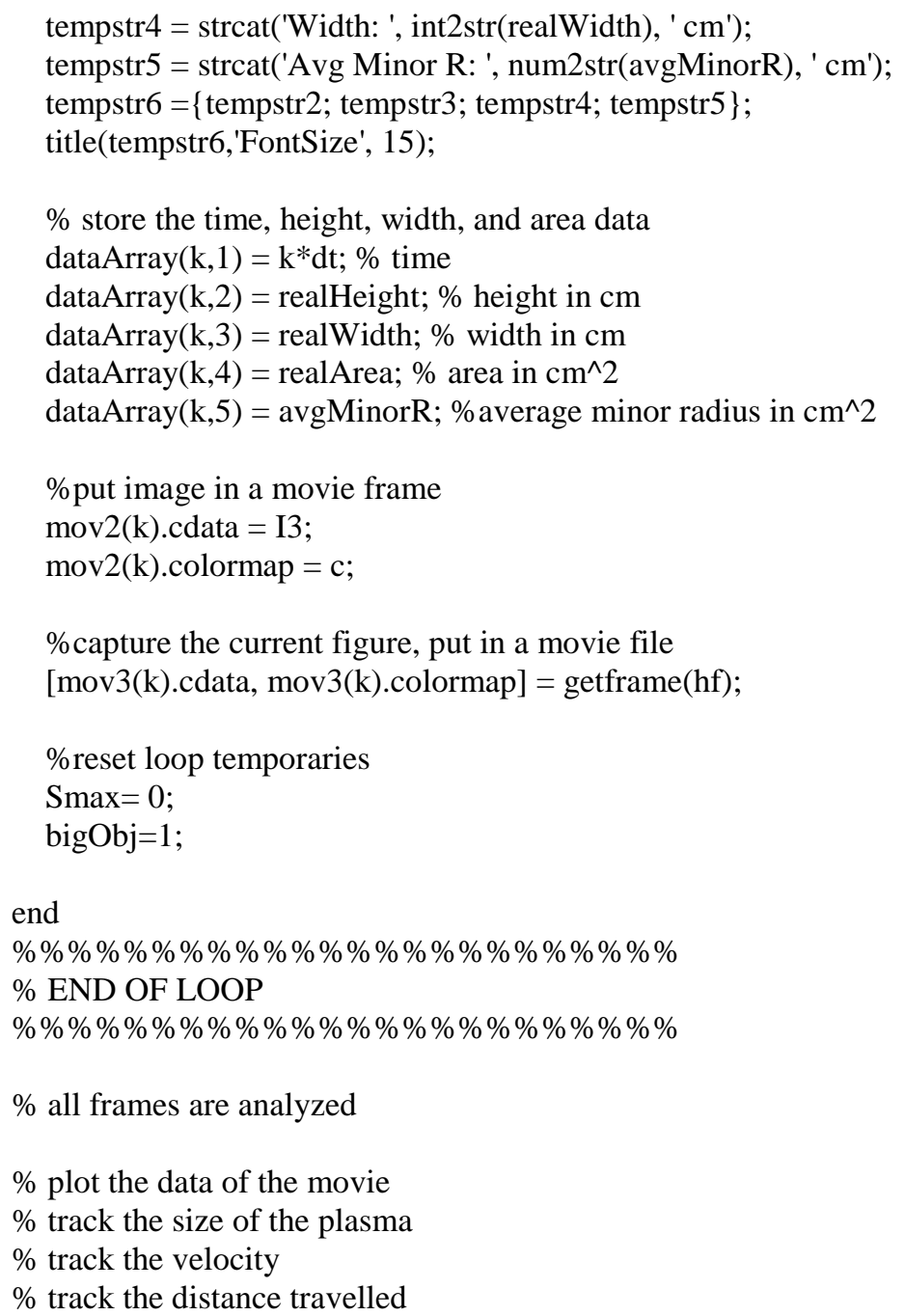

figure(2);

plot(dataArray(:,1), dataArray(:,2));

xlabel('Time (us)');

ylabel('Height $(\mathrm{cm})$ ');

figure(3);

plot(dataArray(:,1), dataArray(:,3));

xlabel('Time (us)');

ylabel('Width (cm)');

figure(4);

plot(dataArray(:,1), dataArray(:,4));

xlabel('Time (us)');

ylabel('Area $\left(\mathrm{cm}^{\wedge} 2\right)$ ');

figure(5); 


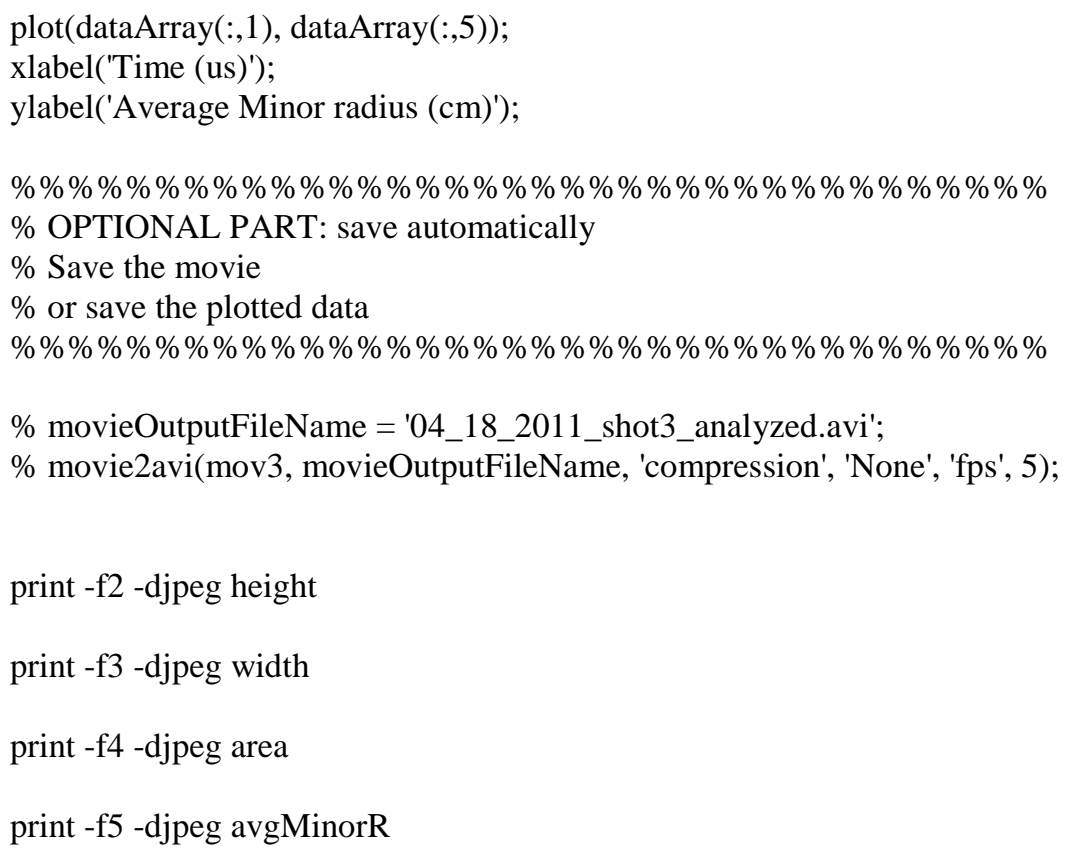

The following are the avgMinorWidth.m and avgMinorHeight.m programs written in MATLAB ${ }^{\circledR}$ to calculate the average minor dimensions of the TAP.

Copyright statement: The program is a copyright of Adam Lodes for this research program. Use of the program by researchers is welcomed as long as credit for authorship is appropriately noted.

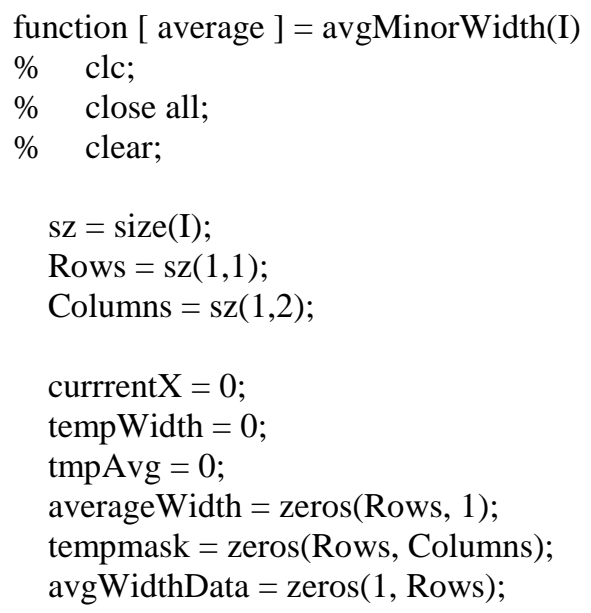




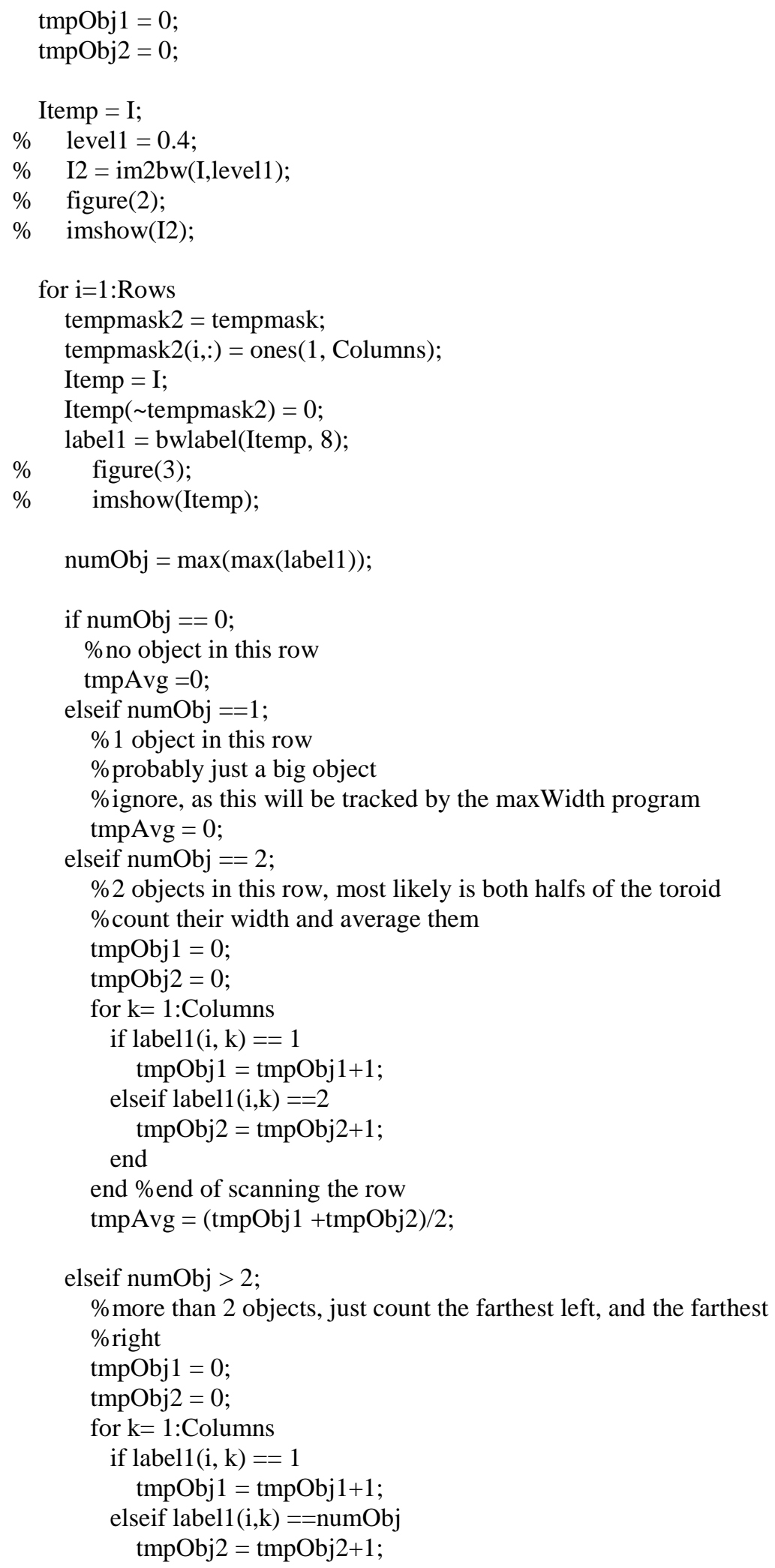




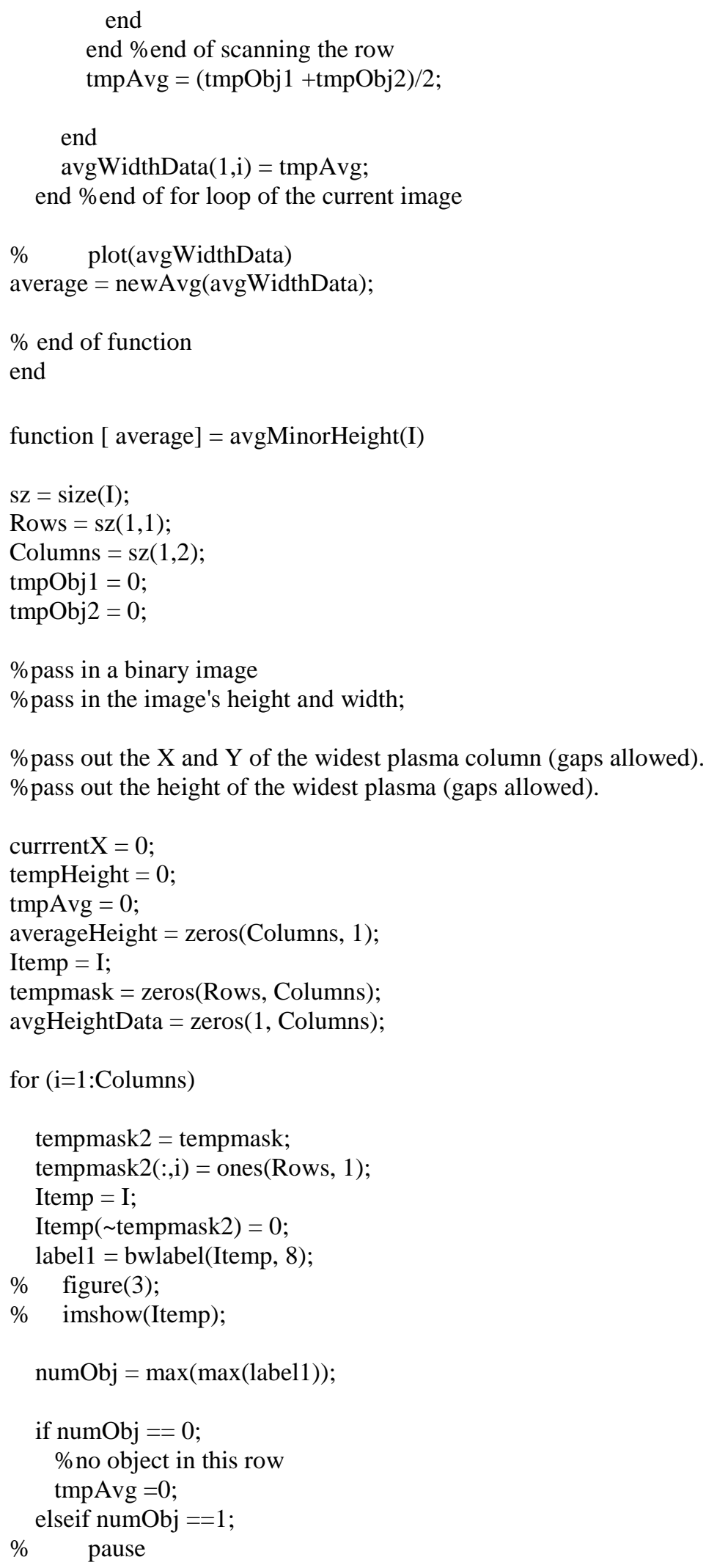


$\% 1$ object in this row

$\%$ probably just a big object

\%ignore, as this will be tracked by the maxWidth program

tmpAvg = 0;

elseif numObj $==2$;

$\%$ pause

$\% 2$ objects in this row, most likely is both halfs of the toroid

$\%$ count their width and average them

tmpObj1 = 0;

$\operatorname{tmpObj} 2=0$;

for $\mathrm{k}=1$ :Rows

if label1 $(\mathrm{k}, \mathrm{i})==1$

tmpObj1 = tmpObj $1+1$;

elseif label1 $(\mathrm{k}, \mathrm{i})==2$

$\operatorname{tmpObj} 2=\operatorname{tmpObj} 2+1$;

end

end \%end of scanning the row

tmpAvg = $(\operatorname{tmpObj} 1+\operatorname{tmpObj} 2) / 2$;

elseif numObj > 2

$\%$ pause

\% more than 2 objects, just count the farthest left, and the farthest

\%right

$\operatorname{tmpObj} 1=0$;

$\operatorname{tmpObj} 2=0$;

for $\mathrm{k}=1$ :Rows

if $\operatorname{label} 1(\mathrm{k}, \mathrm{i})==1$

tmpObj1 = tmpObj1+1;

elseif $\operatorname{label1}(\mathrm{k}, \mathrm{i})==$ numObj $\operatorname{tmpObj} 2=\operatorname{tmpObj} 2+1$;

end

end \%end of scanning the row

tmpAvg = (tmpObj1 +tmpObj2)/2;

end

$\operatorname{avgHeightData}(1, \mathrm{i})=\operatorname{tmpAvg}$;

end

average = newAvg(avgHeightData $)$;

$\%$ end of function

end 


\section{APPENDIX D: PLASMA FIXTURE DRAWINGS}

The plasma generation test stand consists of three parts: the test fixture, the electrical fixtures, and the base. The test fixture and electrical fixtures can be removed from the base to be cleaned and reconfigured for each experiment. The base was designed to allow for the test fixture to align with other equipment (e.g. the shock tube, diagnostic equipment, etc.). The elaborate geometry and spacing of the electrical fixtures were designed to minimize electrical arcing and dielectric surface flashover, providing reliable, quality electrical connections throughout the various experiments. The design and construction of the test fixture and the electrical fixtures depend on the particular experiment. The assembly was required to be mechanically robust to withstand the full impact of the shock wave. The typical assembly used is described in Section 3.3 and is shown assembled in Figure D.1 and in a diagram in Figure D.2.

This assembly was cleaned after each day's experiments. In addition, the wall of the tube of the test fixture, which houses the wire, was lined with a thin sheet of mylar. This plastic acted as a liner to protect the inner wall of the test fixture from carbon deposits from the plasma discharge. The plastic liner was removed and replaced to provide a clean surface for each experiment.

The following are CAD drawings of the plasma fixture assembly. All units are in centimeters. The fixture was designed in a modular fashion for alternate experimentation as discussed in Chapter 6. 


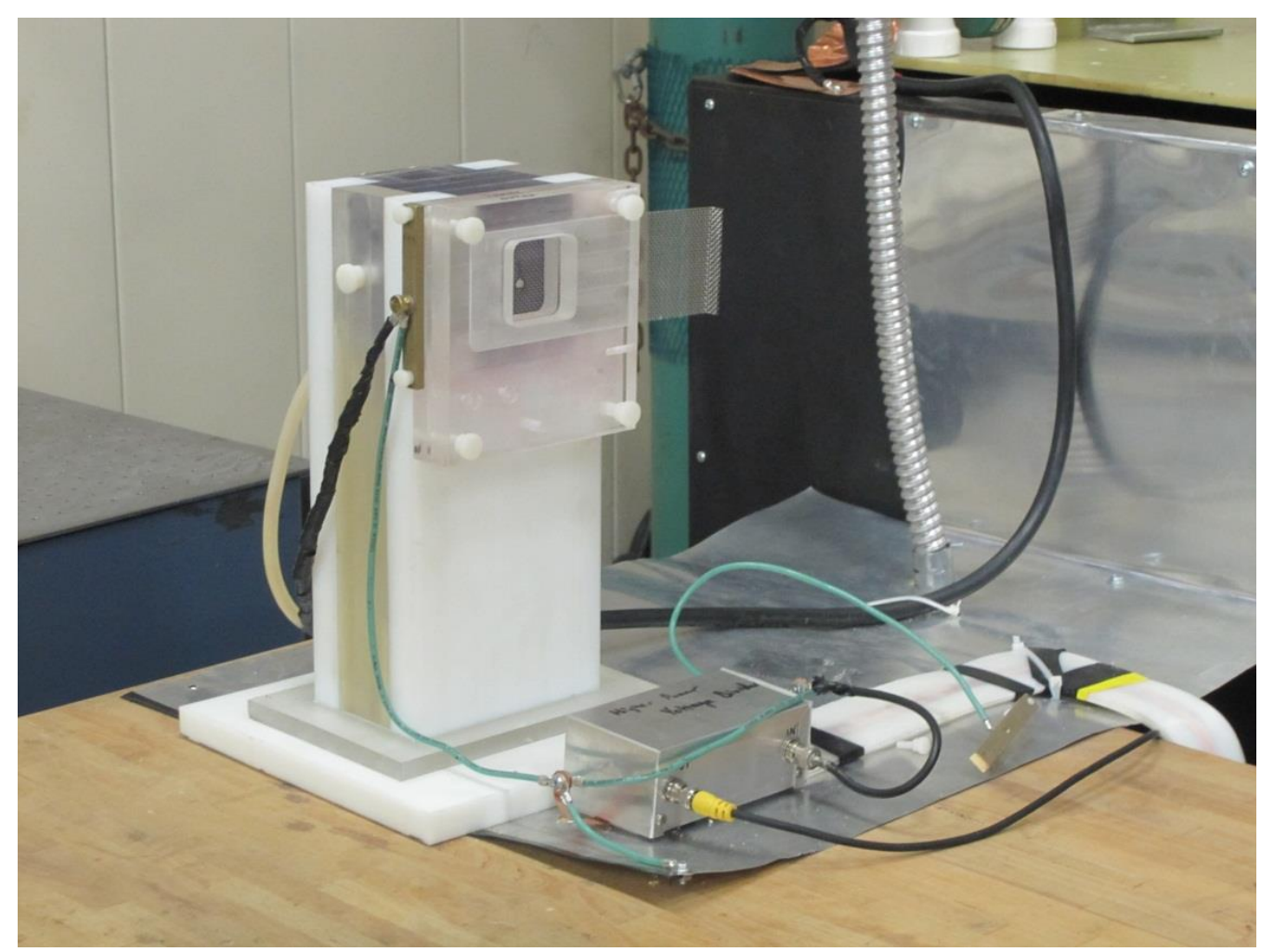

Figure D.1 Plasma fixture assembly

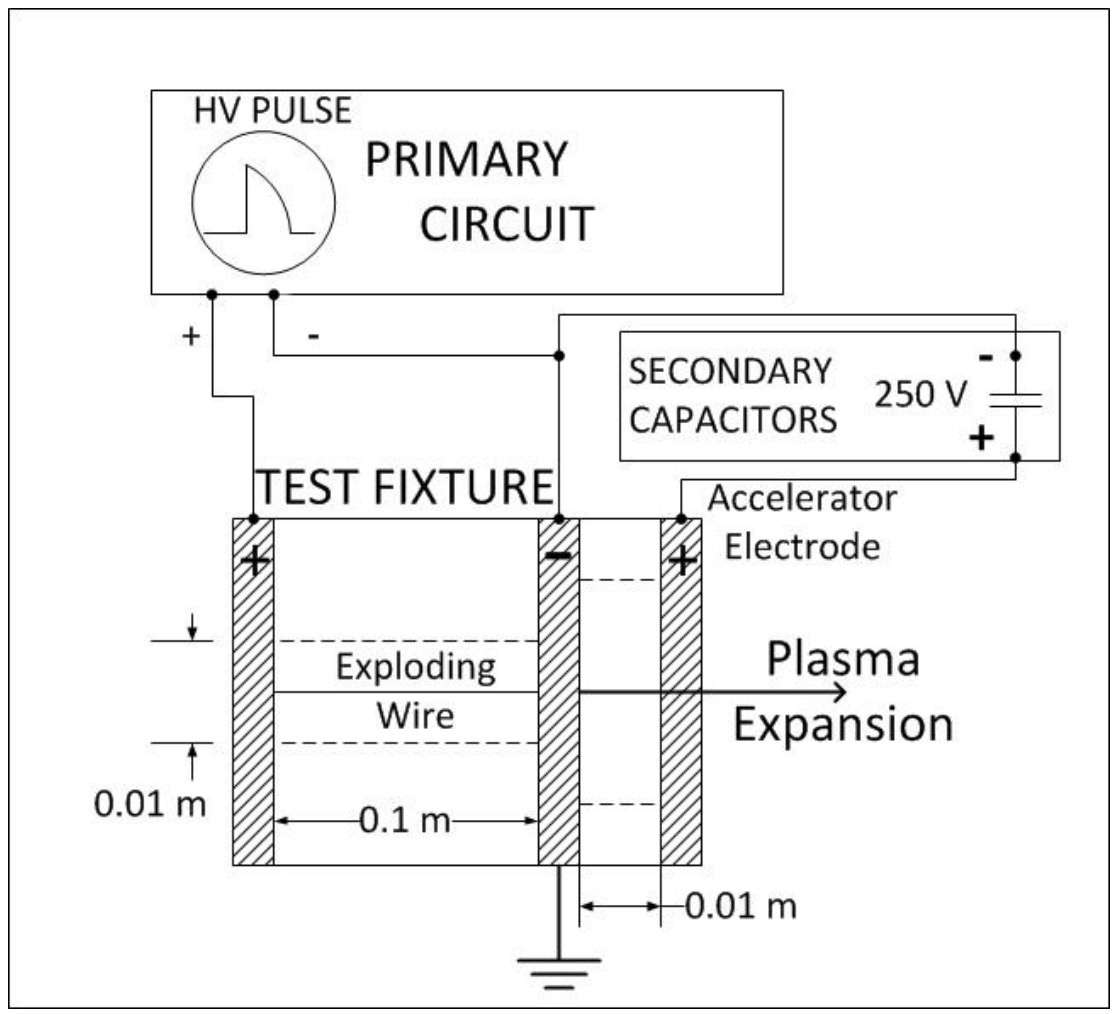

Figure D.2 Toroidal air plasma fixture geometry 

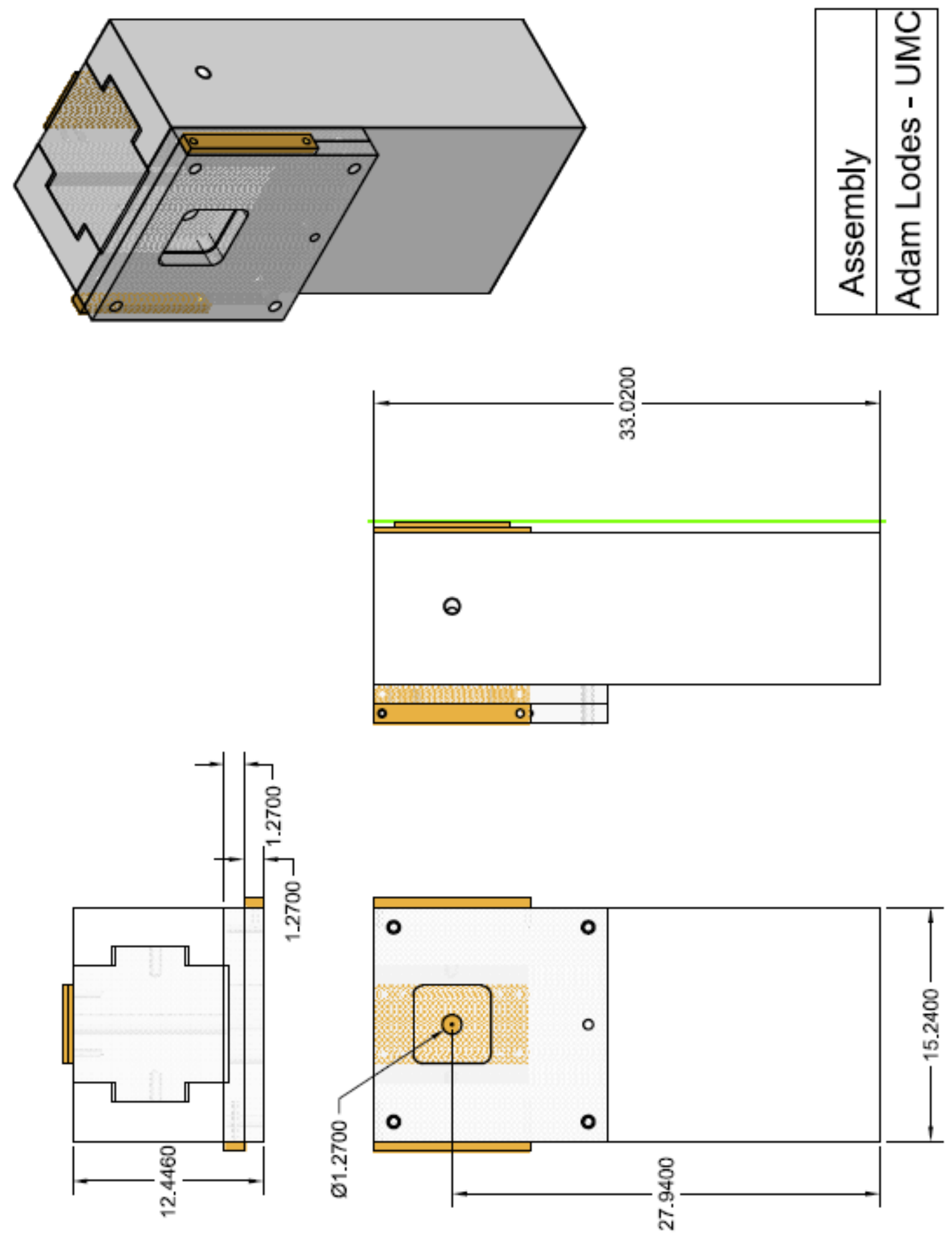

Figure D.3 Drawing of plasma fixture assembly (units in $\mathrm{cm}$ ) 

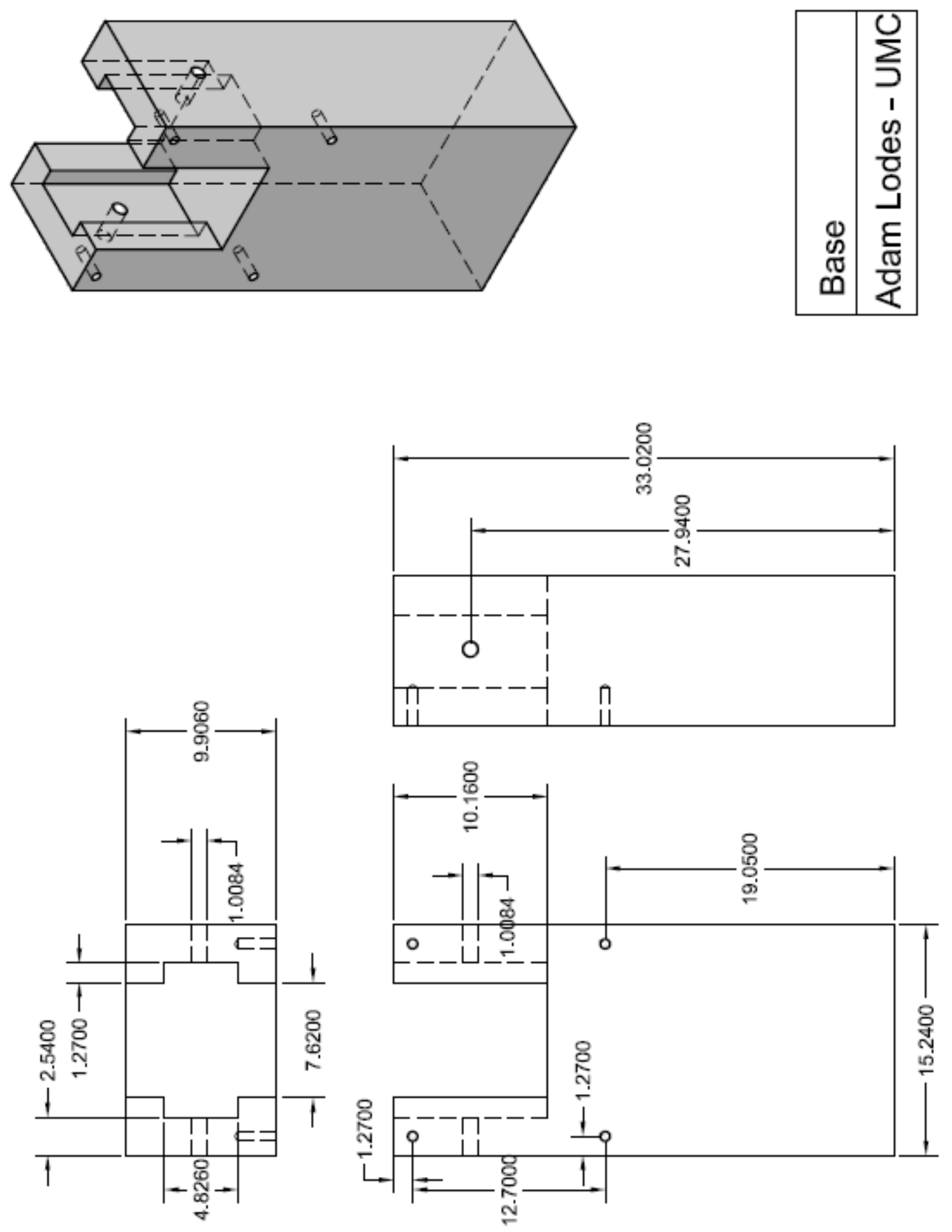

Figure D.4 Drawing of base of plasma fixture assembly (units in $\mathrm{cm}$ ) 


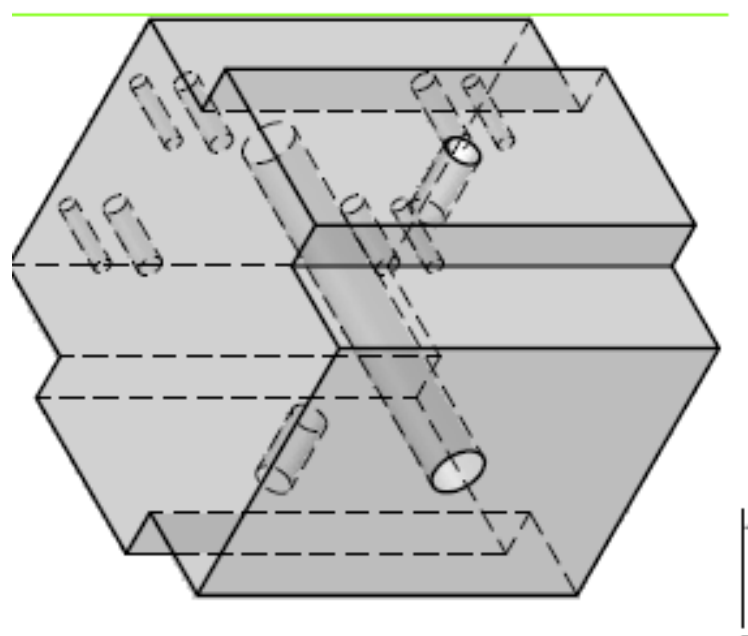

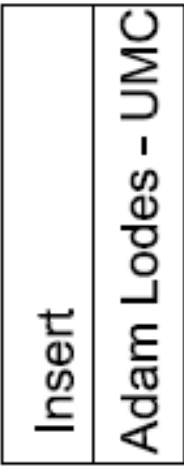
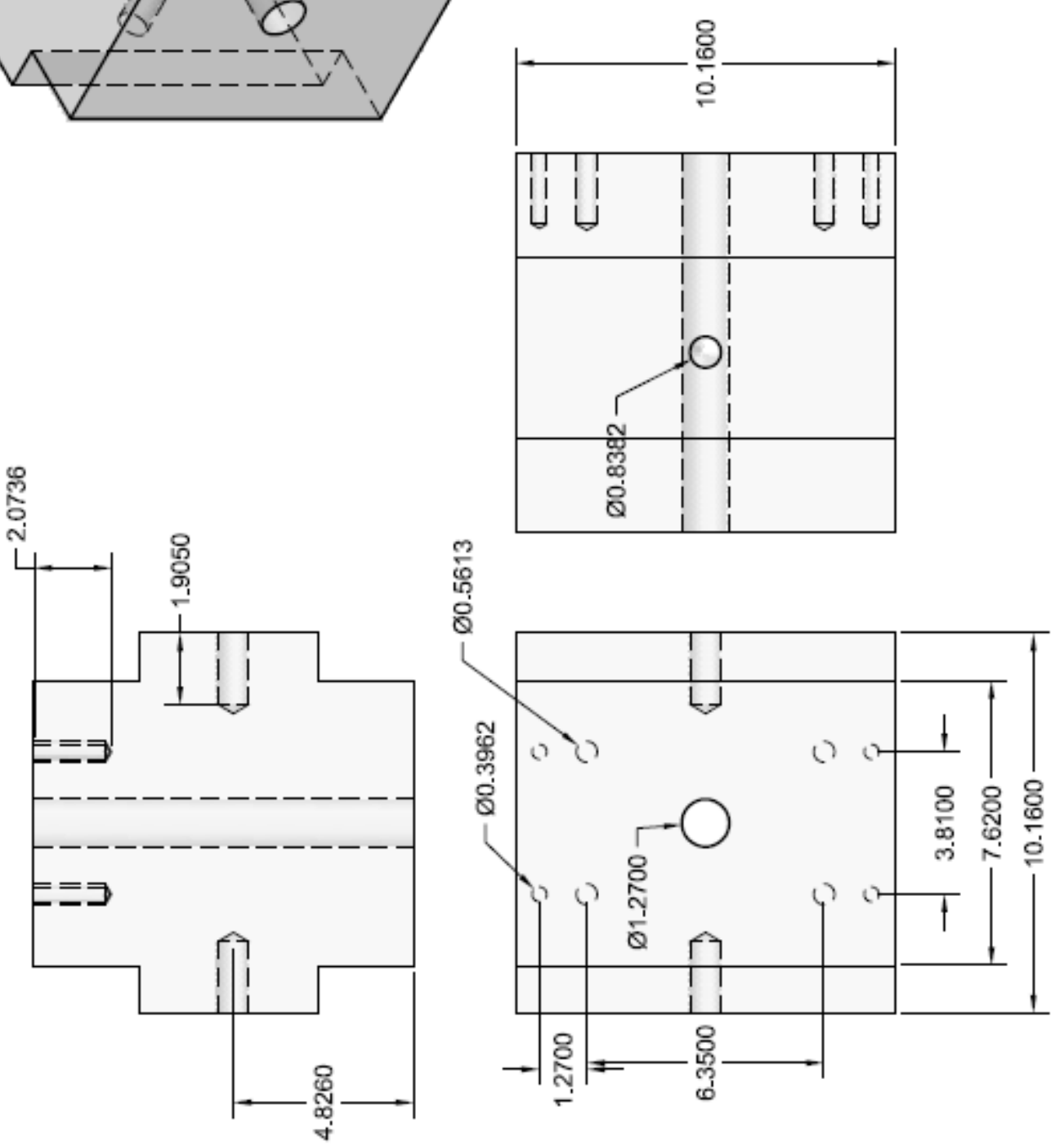

Figure D.5 Drawing of insert of plasma fixture assembly (units in cm) 

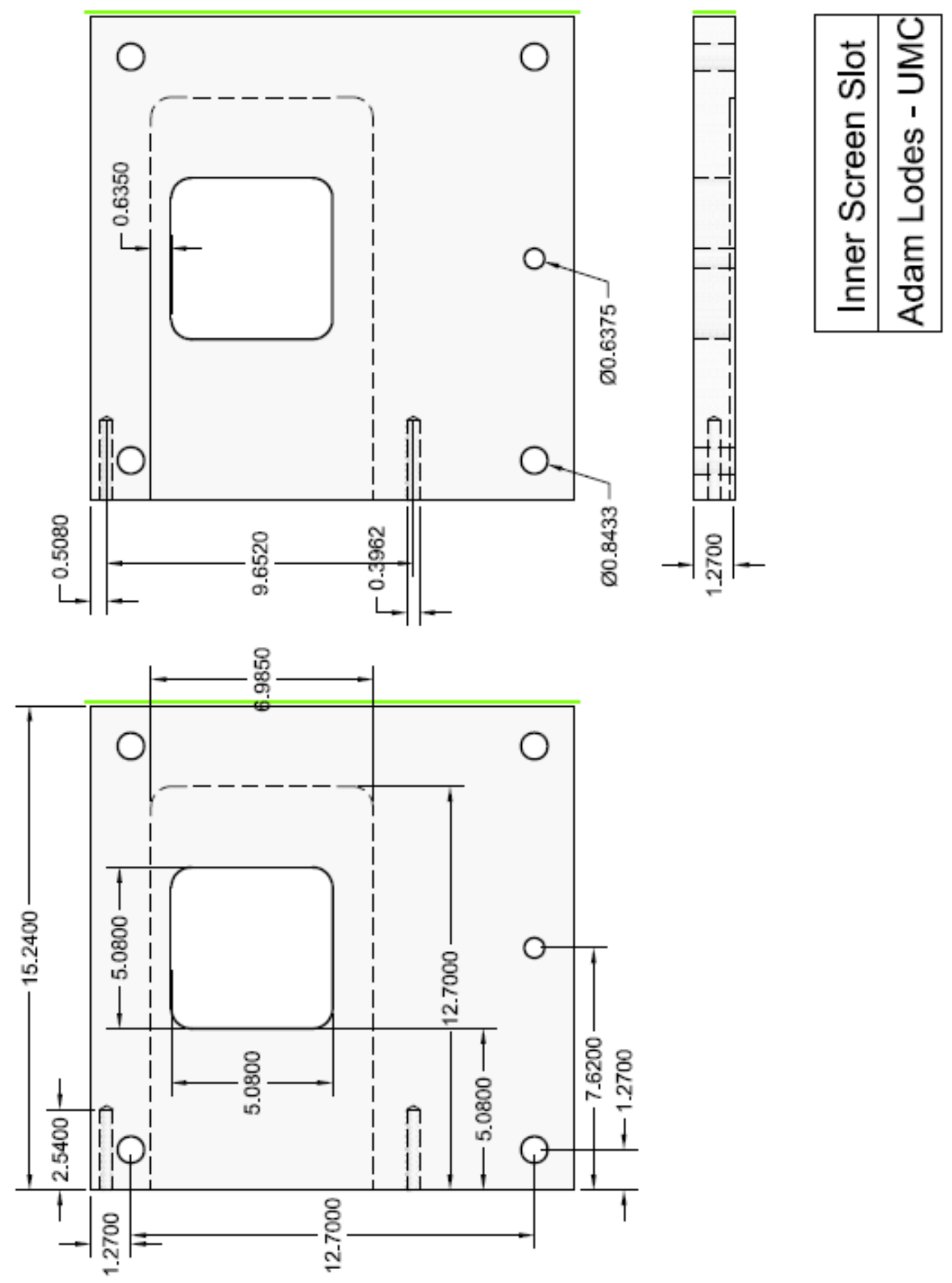

Figure D.6 Drawing of inner screen slot of plasma fixture assembly (units in cm) 


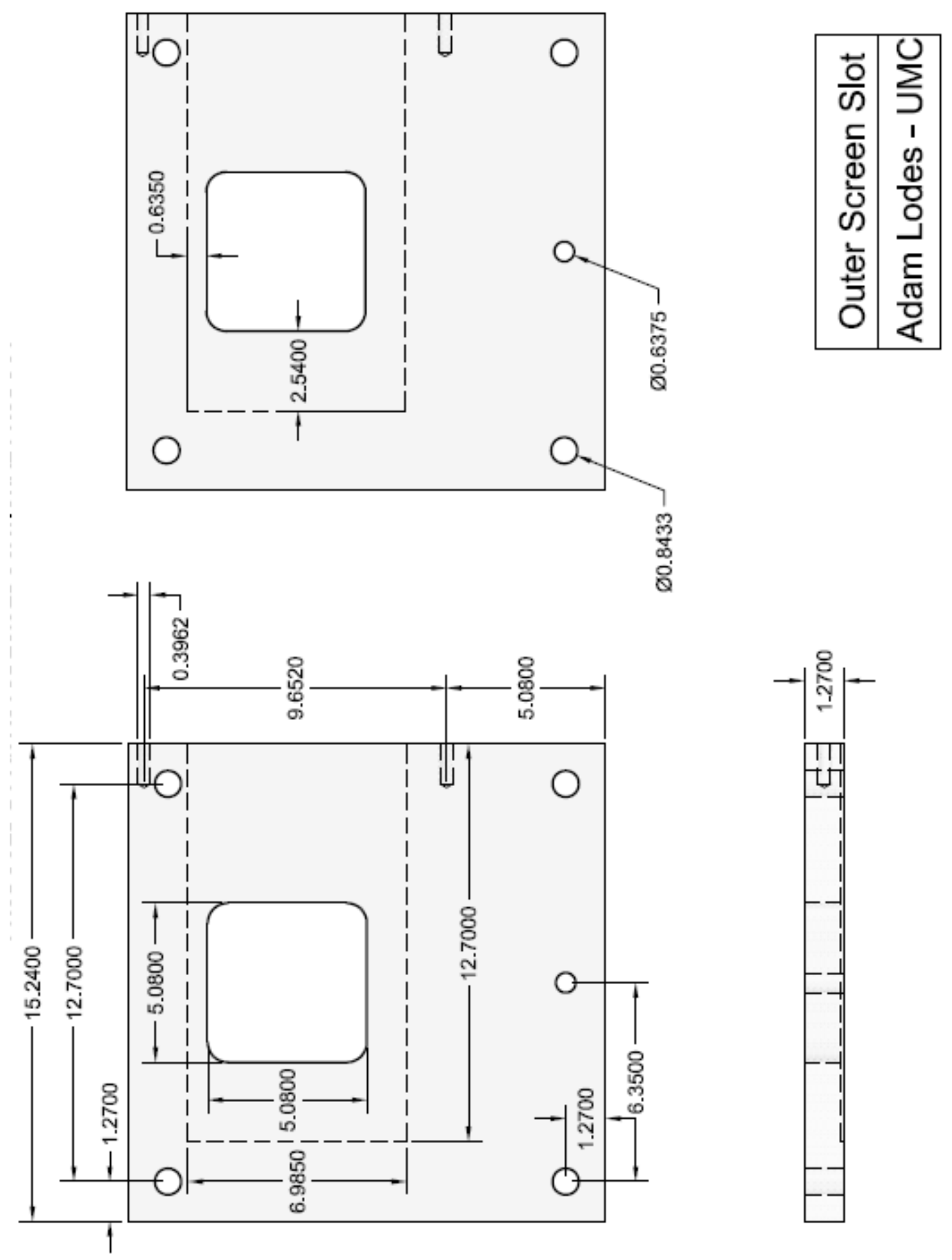

Figure D.7 Drawing of outer screen slot of plasma fixture assembly (units in cm) 


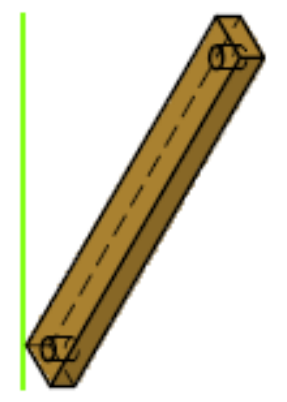

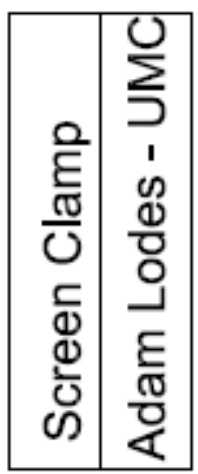
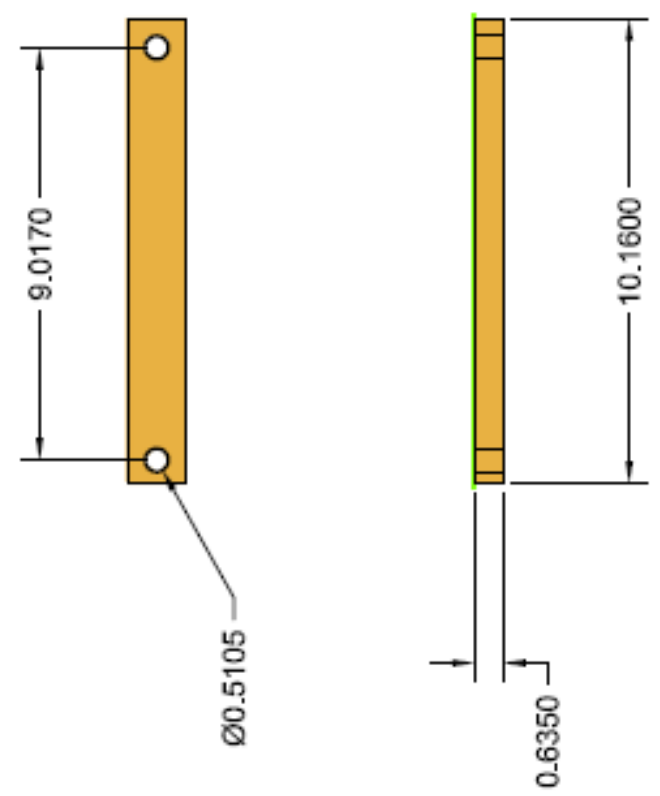

Figure D.8 Drawing of screen clamp of plasma fixture assembly (units in cm) 


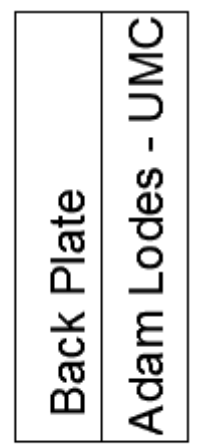

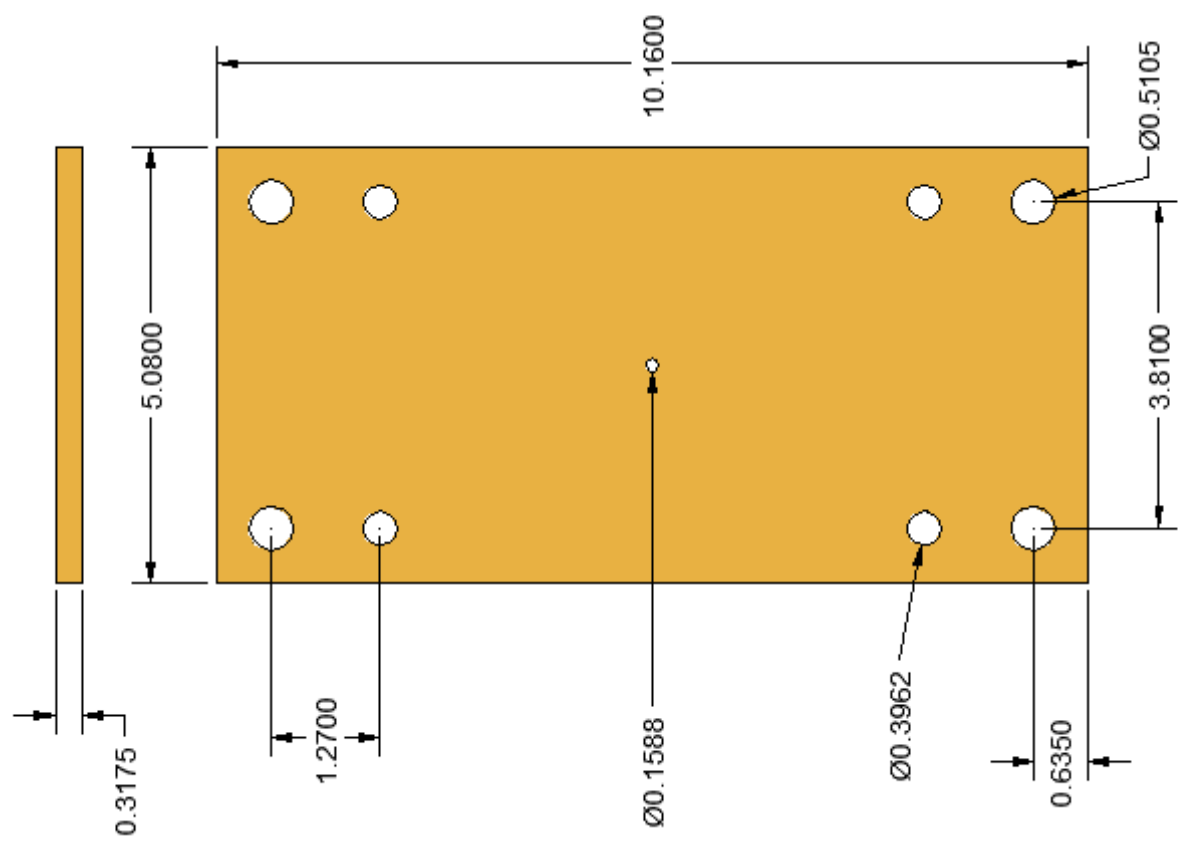

Figure D.9 Drawing of back plate of plasma fixture assembly (units in cm) 


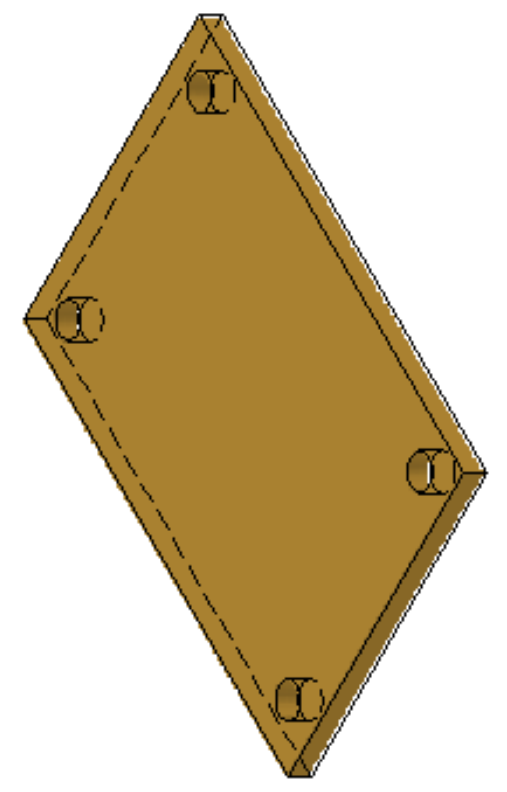

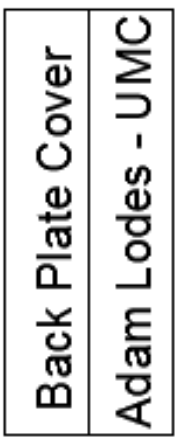

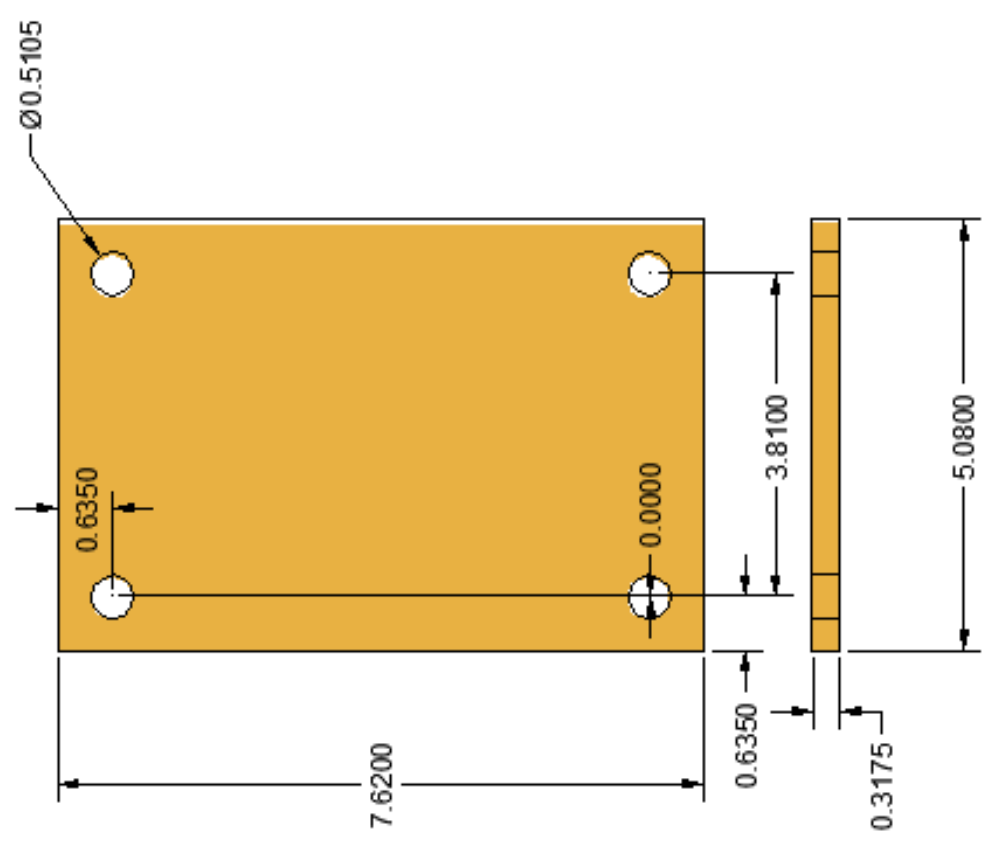

Figure D.10 Drawing of back plate cover of plasma fixture assembly (units in $\mathrm{cm}$ ) 


\section{APPENDIX E: PRESSURE WAVEFORM ANALYSIS}

The pressure waveforms presented in Chapter 8 were processed by a MATLAB ${ }^{\circledR}$ program to minimize the effect of RF noise and to correct for variations in the shock wave. As discussed in Chapter 8, the experiments consisted of nine shock-wave-only and eight interaction trials, with each trial having two channels of data (besides the final two interaction trials, when one of the pressure sensors failed). In addition to these two channels (recorded with two Endevco 8530C-15 pressure sensors), a Kistler 603B1 pressure sensor observed each pressure front inside the shock tube.

When examining a single waveform, RF noise is obvious to detect and filter as required. In order to automate this type of correction, the MATLAB ${ }^{\circledR}$ function smooth was utilized to apply a moving average filter. The filter is a low-pass filter with coefficients equal to the reciprocal of the span. A span of 13 was chosen to minimize the effects of RF noise but keep the integrity of the pressure waveforms. As an example for comparison, Figure E.1 is an unsmoothed pressure waveform of the shock wave inside the shock tube as observed by a Kistler 603B1 pressure sensor. Figure E.2 is the processed version of the same pressure data. As shown, the noise signals are considerably decreased in amplitude due to the processing.

After filtering the RF noise, the waveforms were corrected for variations in the shock wave. Each pressure waveform was corrected by a normalization factor based on the small variances in the shock front peak amplitude from the Kistler sensor (inside the tube). 
Then for comparison purposes, the data from the shock-wave-only trials were averaged together, per channel. The average shock wave amplitude without interaction of the plasma was 7.73 psi for Channel 3, and 5.85 psi for Channel 4. The analysis for amplitude reduction presented in Chapter 9, in Figure 9.6 and Table 9.1, is computed by comparing each channel of interaction data with this averaged data.

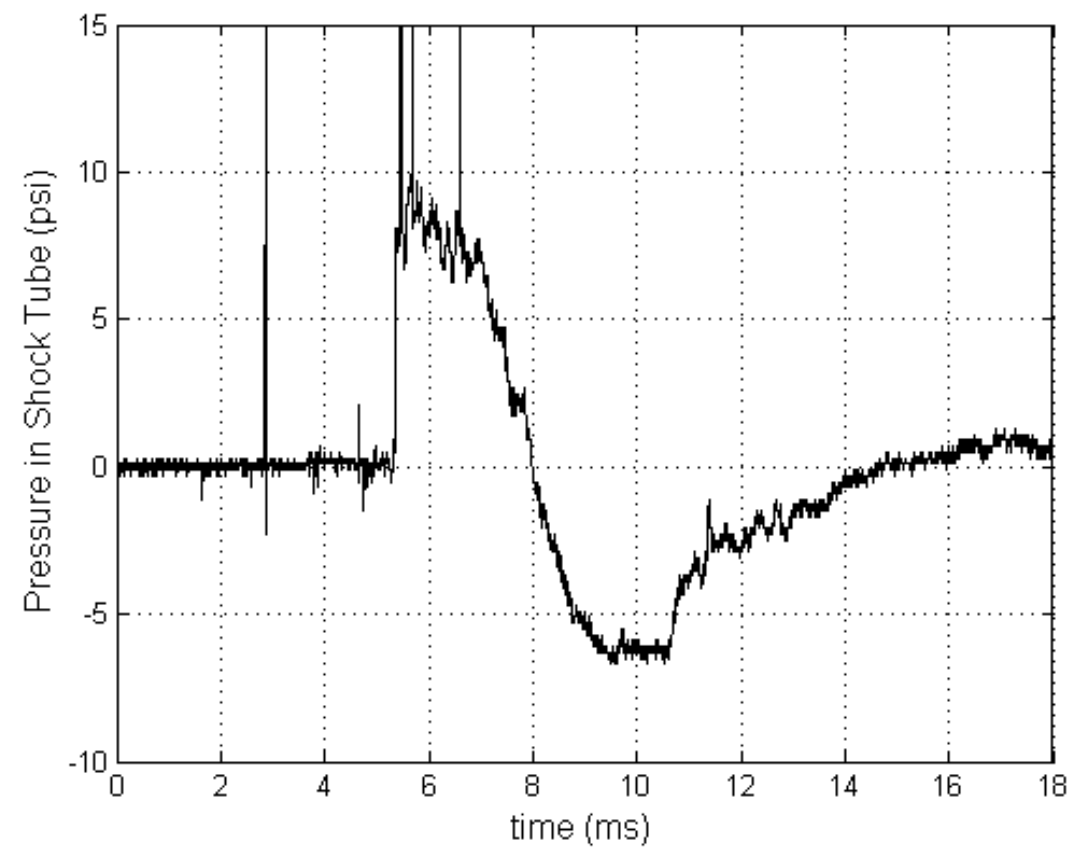

Figure E.1 Example unprocessed pressure waveform of the shock wave inside the tube 


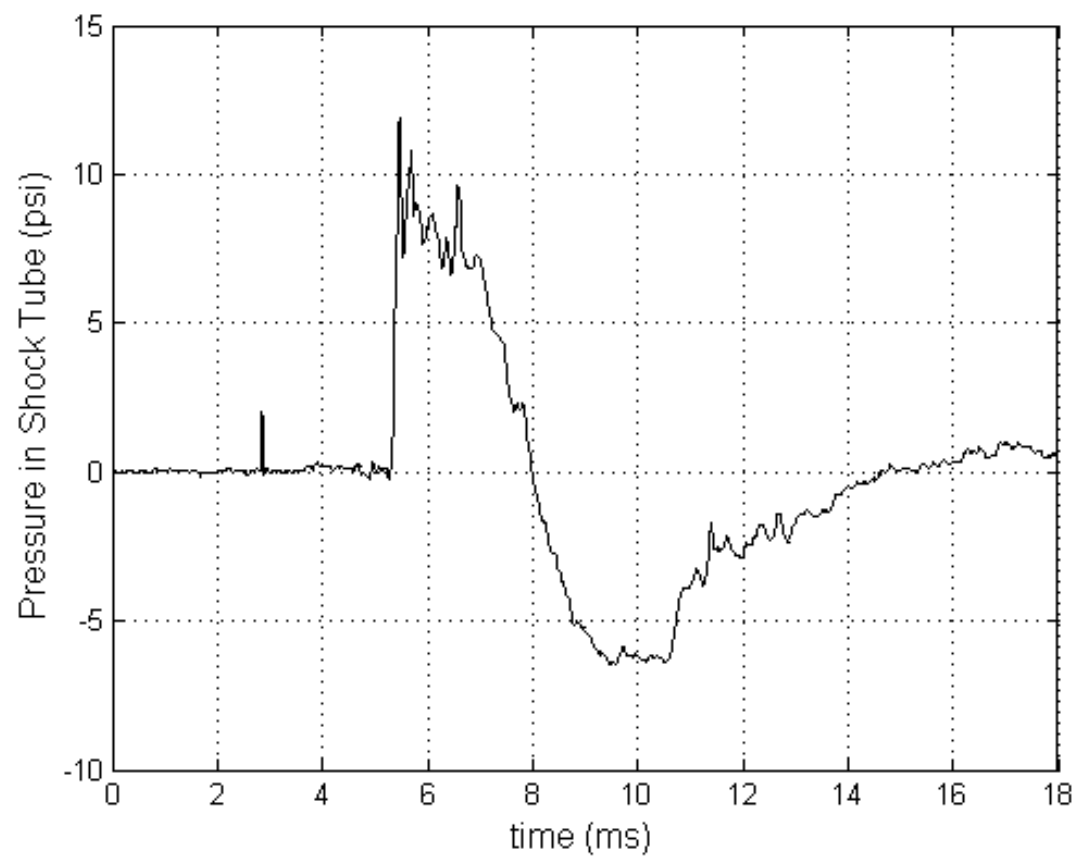

Figure E.2 Example filtered pressure waveform of the data presented in Figure E.1 


\section{VITA}

Adam Lodes earned the three degrees of Bachelor of Science in Electrical Engineering, Computer Engineering, and Mathematics from the University of Missouri in December of 2007. He first began research in the field of pulsed power with Dr. Randy Curry in 2004 running experiments on the pulsed electric field inactivation of bacteria in drinking water as an undergraduate.

Upon entering graduate school at the University of Missouri, Adam Lodes researched atmospheric pressure plasma discharges for his $\mathrm{PhD}$. His graduate research program provided him with experience in air plasma sources, plasma spectroscopy, plasma diagnostics, and pulsed power systems. To this date Adam has presented at four international conferences. Adam earned his Doctor of Philosophy in Electrical and Computer Engineering in December of 2013. He can be reached at his permanent email address: AdamLodes@gmail.com. 